\title{
Variability in the North Atlantic Deep Western Boundary Current: upstream causes and downstream effects as observed at Line $\mathrm{W}$
}

by

\author{
Beatriz Peña-Molino
}

B.Sc, Universidad de las Palmas de Gran Canaria, Spain (2003)

Submitted in partial fulfillment of the requirements for the degree of

Doctor of Philosophy in Physical Oceanography

at the

MASSACHUSETTS INSTITUTE OF TECHNOLOGY

and the

WOODS HOLE OCEANOGRAPHIC INSTITUTION

September 2010

(c) Beatriz Peña-Molino, 2010. All rights reserved.

The author hereby grants to MIT and to WHOI permission to reproduce and distribute publicly paper and electronic copies of this thesis document in whole or in part.

Author

Joint Program in Physical Oceanography Massachusetts Institute of Technology Woods Hole Oceanographic Institution July 27, 2010

Certified by .

Terrence M. Joyce

Senior Scientist, Woods Hole Oceanographic Institution

Thesis Supervisor

Accepted by

Karl R. Helfrich

Chair, Joint Committee for Physical Oceanography 


\title{
Variability in the North Atlantic Deep Western Boundary \\ Current: upstream causes and downstream effects as observed at Line W
}

by

Beatriz Peña-Molino

\begin{abstract}
Submitted to the Joint Program in Physical Oceanography - Massachusetts Institute of Technology / Woods Hole Oceanographic Institution on July 27, 2010, in partial fulfillment of the requirements for the degree of Doctor of Philosophy in Physical Oceanography
\end{abstract}

\begin{abstract}
The variability in the DWBC, its connection to the forcing in the northern North Atlantic and interaction with the Gulf Stream were explored from a combination of remote sensing and in-situ measurements in the western North Atlantic.

Using satellite altimetry and Sea Surface Temperature (SST) we found evidence of the relation between changes in the Gulf Stream path and the variability in the temperature and velocity fields in the Slope Water. This relation was such that southward shifts of the main axis of the Gulf Stream were preceded by cold temperature anomalies and intensification of the southwestward flow.

The analysis of 5.5 years of moored CTD and horizontal velocity data in the DWBC at $69^{\circ} \mathrm{W}$ recorded during the period 2002-2008, showed that the variability along the DWBC is linked to changes in the dense water formation regions. The evolution of potential vorticity (PV) at the mooring site, characterized by a transition from deep to upper Labrador Sea Water (LSW), was similar to that observed in the Labrador Sea 6 to 9 years earlier, and imply spreading rates for the LSW that varied over time from 1.5 to $2.5 \mathrm{~cm} / \mathrm{s}$. The time dependence of the spreading rates was in good agreement with changes in the strength of the DWBC at the mooring site.

The evolution of the DWBC transport was explored in more detail from a 5element moored array, also at $69^{\circ} \mathrm{W}$. The results, for the period of 2004-2008, were consistent with the single mooring analysis. The variability measured from the array showed that upper, intermediate and deep water mass layers expand and contract at each other's expense, leading to alternating positive and negative PV anomalies at the upper-LSW, deep-LSW and Overflow Water (OW). Larger DWBC transports were associated with enhanced presence of recently ventilated upper-LSW and OW, rather than deep-LSW. The relative contribution of the different water masses to the
\end{abstract}


observed circulation was investigated by inverting individual PV anomalies isolated from the observations. We found that changes in the depth-integrated circulation were mostly driven by changes in the OW.

Thesis Supervisor: Terrence M. Joyce

Title: Senior Scientist, Woods Hole Oceanographic Institution 


\section{Acknowledgments}

First I want to thank my advisor, Terry, for his guidance, his patience, for being so generous both with his time and his ideas, at sea and in land, and for teaching me something I could not find in the books, and that is to be enthusiastic about my work. I am very very grateful for these years.

I want to say thanks to my committee. To John Toole, who has been a very important part of my education here. He has always found the time to answer my questions. I have learned so much from him, also at sea and in land. Thanks for so many great conversations John. To John Marshall. It was a lot of fun to "think PV" with you. Thanks for teaching me that simpler is better. To Mike Spall and Bob Pickart; they have always been extremely generous with their time and ideas too. And thanks to the chair of the defense, Amy Bower. She joined us at the end of the journey, but still contributed to my thesis in many ways, with great fresh ideas and with very valuable advise. Thanks Amy.

My thesis would have certainly not be possible without the work of many others at WHOI. The WHOI Subsurface Mooring Operation Group: Scott Worrilow, Brian

Hogue, Dan Borgoff and other occasional contributors. Ruth Curry, Dan Torres, Jane Dunworth and Dave Wellwood they all played very important roles in my work here. Thanks to so many people in the Physical Oceanography department at WHOI that made my time here so much more enjoyable. Annie and Hazel, and the rest of the administrative personnel, that takes care of so many things for us, so that we don't have to.

To Academic Programs Office, so so so many thanks, for taking such good care of me in so many different ways. For always being in my side (whatever side that was...). Over the years they have really become much more than what their job titles say, they have become my friends. Thanks Marsha, Julia, Valerie, Christine and Michelle. And lots of thanks to Jim Yoder and Jim Price, both of which have gone such a long way to offer me numerous teaching opportunities. They really made my experience 
here so much more complete. Big thanks to the MIT side. To Ronni and Carol and so many other at MIT that made sure the program stays "joint".

And I could not go without saying thanks to my friends. To my classmates at WHOI and MIT, Katie, Brian, Jessica, Claude, Kjetil and Tatiana among others. To so many others that were here before me Matt, Hristina, Yohai, Ariane, Stephanie, Carlos, Dave, Melanie and Greg, and to the ones that will still be here when I go, Nick and Rachel (my office-mates during this last stretch, thanks for being so nice through my "darkest moments"), Evgeny and Jimbo, Rebecca, Wilken, Julian and everybody else. And thanks to those other friends, who I did not speak science with. They really help keeping things real as work was getting more complicated. Brian, Casey, Dara, Em, Joanna, Kevin, Sam and Stepth among others, thanks for making Woods Hole my home.

And last but not least, I want to say GRACIAS to my family, for so many things that will not fit in these few lines. For their unconditional support, their patience, for being so proud of me and for teaching me almost everything else but Physical Oceanography. For making me laugh so much and so hard. They really are my favorite people, and I wanted to dedicate this thesis to them. Muchas muchas gracias.

During the first two years, my time here was funded through a La Caixa Foundation fellowship as well as an MIT presidential fellowship. Financial support for my thesis work was provided by National Science Foundation grants OCE-0241354 and OCE-0726720. Support for traveling to meetings was also provided by the MIT Houghton Fund and the WHOI Academic Programs Office. 


\section{Contents}

1 Introduction 11

1.1 Background . . . . . . . . . . . . . . . . . . . . . . 12

1.2 Thesis outline . . . . . . . . . . . . . . . . . . 16

2 Surface variability in the Slope Water and its relation to the Gulf $\begin{array}{ll}\text { Stream path } & 19\end{array}$

2.1 Introduction . . . . . . . . . . . . . . . . . . . . . . 19

2.2 Data . . . . . . . . . . . . . . . . . . . . . . . 21

2.2 .1 Remote sensing . . . . . . . . . . . . . . . . . . . 21

$2.2 .2 \quad$ Hydrographic data . . . . . . . . . . . . . . . . . 22

2.3 The surface signal. Part I: SST and the Gulf Stream PC1 lag-correlation 23

2.4 The surface signal. Part II: modes of surface velocity and SST variability 33

2.5 The origin of the anomalies $\ldots \ldots \ldots \ldots \ldots$

2.6 The vertical structure . . . . . . . . . . . . . . . . . . . . . . . . . . . . . . 38

2.7 Summary and discussion $\ldots \ldots \ldots \ldots$

3 Recent changes in the Labrador Sea Water within the Deep Western Boundary Current Southeast of Cape Cod 45

3.1 Introduction . . . . . . . . . . . . . . . . . 45

3.2 The dataset: W3 deployment. . . . . . . . . . . . . . . . . 49

3.3 Evolution of the hydrographic properties and circulation at W3 . . 52

3.3.1 The mean and the anomaly fields. . . . . . . . . . . . . . 52 
3.3.2 Vertical modes of co-variability. . . . . . . . . . . . 55

3.4 The transport response to changes in the LSW . . . . . . . . . 62

3.5 Water mass variability . . . . . . . . . . . . . . . 65

3.5.1 $\theta / \mathrm{S}$ variability $\left(\right.$ fixed $-\gamma_{n}$ range) $\ldots \ldots \ldots \ldots \ldots$

3.5.2 Density variability in the water masses. . . . . . . . . . 67

3.6 The fate of the LSW . . . . . . . . . . . . . . . . 70

3.7 Summary and discussion $\ldots \ldots \ldots \ldots \ldots \ldots$

4 Variability in the DWBC, local vs. remote forcing 79

4.1 Introduction . . . . . . . . . . . . . . . . . . . . . . . 79

4.2 The data set . . . . . . . . . . . . . . . . . . . . . . . . . 82

4.2 .1 The Line $\mathrm{W}$ array . . . . . . . . . . . . . . . . . . . . 82

4.2.2 Constructing an objectively mapped section . . . . . . . . 85

4.3 Rings, meanders and the mean circulation at Line $\mathrm{W}$. . . . . . . 89

4.4 Energetics of the flow . . . . . . . . . . . . . . 96

4.5 Water mass changes and the response in the circulation. . . . . . 103

4.6 Intermediate and deep water transport . . . . . . . . . . . . 107

4.7 The winter 2007-2008 rapid event . . . . . . . . . . . . . 110

4.8 Summary and discussion . . . . . . . . . . . . . . . . . . . . . . 114

5 The response of the circulation to deep potential vorticity anomalies: A PV-inversion approach with applications to the North Atlantic $\begin{array}{ll}\text { DWBC } & 119\end{array}$

5.1 Introduction . . . . . . . . . . . . . . . . . . . . . . . 119

5.2 The machinery: a PV inversion. . . . . . . . . . . . . . . . . . . 122

5.2 .1 A simplified PV equation. . . . . . . . . . . . . . 123

5.2.2 The elements of the problem: an idealized representation of the western North Atlantic . . . . . . . . . . . . . . . 127

5.3 An infinite basin with constant stratification . . . . . . . . . . 130

5.4 The boundary value problem . . . . . . . . . . . . . . . 133 
5.4.1 The constant stratification case . . . . . . . . . . . . 135

5.4 .2 A Slope Water stratification profile . . . . . . . . . . . . . 140

5.4.3 The combined effect of LSW and OW PV anomalies . . . . . . 141

5.4.4 The effect of the mean Gulf Stream . . . . . . . . . . . . . . . 146

5.5 Summary and discussion . . . . . . . . . . . . . . 146

6 Discussion 


\section{Chapter 1}

\section{Introduction}

The western North Atlantic is one of the most intensively studied regions in the World Ocean. It comprises two very different large scale current systems: the Gulf Stream a poleward flowing baroclinic jet that is surface intensified, but has relatively strong velocities all the way to the bottom, and the Deep Western Boundary Current (DWBC) an equatoward flowing current with bottom intensified velocity cores embedded in a relatively uniform flow (eg. Joyce et al. (2005)). The mechanisms that drive the time mean flow in both of these current systems are relatively well known, and can be explained by the existing theories for the steady circulation of the ocean (eg. Stommel (1948) and Stommel and Arons (1960)). However, in the context of a changing climate, the question of what drives the variability is the more relevant one. Our understanding of the variability, at the present time, is much less complete. Due to the lack of long term measurements, our knowledge is heavily based on modeling studies, whose results for interannual and decadal (or longer) variability are not always consistent and yet to be validated. Nevertheless, next, a summary of what the current view of variability in the North Atlantic is provided. A more detailed introduction to some of the elements of the problem, will be given at the beginning of each chapter. 


\section{$1.1 \quad$ Background}

Many of the elements of the North Atlantic circulation appeared to be related to the atmospheric forcing, whose dominant mode of variability is described by the North Atlantic Oscillation (NAO, see Hurrell (1995)). Periods of high (positive) NAO index are characterized by stronger than usual westerlies and are also typically associated with large air-sea fluxes (from the ocean to the atmosphere) in the northern North Atlantic. Both of these, winds and thermohaline forcing, are the main drivers of the Atlantic Meridional Overturning Circulation (MOC), a two dimensional (vertical and meridional) cell that carries, at depth, cold waters from high latitudes to low latitudes, and brings warmer, near surface waters northward to replace them. Because the temperature gradients in the ocean are large both in the vertical and meridional direction, the MOC plays a fundamental role in the meridional heat transport.

In this two dimensional flow of cold/warm water the vertical component associated with the sinking of cold water at high latitudes can take place both in the Subpolar and Subarctic basins, where large wintertime heat fluxes lead to the densification of surface waters and their subsequent convective mixing. In the Labrador Sea, observations have shown that the volume as well as the properties of the water that result from convection every year, are related to the strength of the atmospheric forcing (eg. Curry and McCartney (2001) and Yashayaev (2007)). During periods of sustained positive NAO, colder, denser and more voluminous layers of Labrador Sea Water (LSW) are formed. When the NAO is low, shallow or no convection is observed. Dense water formation in the Nordic Seas is also controlled, at least in part, by wintertime heat fluxes (Bacon (1998)). However, the balance between the inflow of fresh water from Arctic origin and saline subtropical water can significantly alter the expected production of deep water based on heat fluxes alone (Dickson et al. (1996)).

Changes in the rate of dense water formation are expected to have an impact on the MOC. This can be seen in a number of models with very different configurations -atmosphere coupled/ocean only, eddy-resolving/non eddy-resolving, isopycnal/Z- 
coordinates, global/area limited- that are forced in a number of different ways. One can imagine that the variety in the answers provided by the models is as large, if not larger, than the variety in the configurations themselves. And in reviewing the very extensive literature one can do little but conclude that an increase in the LSW production can lead to an increase, a decrease or a zero net change of the MOC with a lag of +3 , 0 or -3 years (eg. Mauritzen and Häkkinen (1999), Koltermann et al. (1999), Eden and Willebrand (2001), Böning et al. (2006), Pickart and Spall (2007), Kohl and Stammer (2008), Zhang (2008)). A little more consensus exists, perhaps, regarding the impact of waters originating in the Greanland-Iceland-Norwegian (GIN) Seas (eg. Gerdes and Koberle (1995), Kohl and Stammer (2008)). It is typically found that enhanced production of deep water in the Nordic Seas leads to the strengthening of the MOC. However, the absence of eddies in these coarse resolution models and the lack of an observational record in the Nordic Seas comparable to the available records at the Labrador Sea, make their results somewhat questionable.

One of the aspects of the problem, and the cause of some recent debate, is related to the export of the dense water. In the traditional view of the circulation of the North Atlantic Deep Water (NADW), built upon theory (Stommel and Arons (1960)) as well as tracer observations (eg. Talley and McCartney (1982) and Smethie (1993)), the DWBC constituted the dominant pathway into the subtropics. The OW originating in the Nordic Seas, circulates around the Labrador Sea in a deep boundary current. While in the Labrador Sea, the OW is modified by entrainment with the surrounding water, and its joined by the LSW at intermediated depths before exiting the subpolar North Atlantic (McCartney (1992)). Over the years, observations at the exit of the subpolar gyre as well as along the continental slope in the western North Atlantic consistently showed the existence of a deep boundary current carrying dense poorly stratified waters southward (eg. Swallow and Worthington (1961), Schott et al. (2004), Pickart and Smethie (1998), Joyce et al. (2005), Toole et al. (2010)), reinforcing the existing view of a continuous DWBC that connects the northern North Atlantic and the subtropics. But more recently, Fischer and Schott (2002) float ob- 
servations, and later work by Bower et al. (2009), both with float trajectories and numerical simulations, suggested that a larger fraction of the LSW that reaches the subtropics does so via an interior pathway. The DWBC played, according to these authors, a secondary role in the export of LSW, mostly due to the eddy variability, unresolved in coarse resolution models.

A number of the studies mentioned above considered the effects of the buoyancy forcing alone, hence the expectation of a linear response of the MOC to changes in the dense water formation rates. However, as we said earlier in the introduction, the wind plays an equally important role. In fact, recent observations suggest that on seasonal and shorter time scales, the wind is the main driver of the Atlantic MOC (Cunningham et al. (2007)). The contribution of the wind to the longer time scales can happen in a number of ways. Changes in the wind patterns can affect convective activity directly by, for instance, advecting cold air over the convective basins (Våge et al. (2009)), or by opening new pathways for the return of saline subtropical waters to high latitudes (Häkkinen and Rhines (2009)). The winds are also fundamental in setting the ocean in motion, and generating a background state that the thermohaline circulation can interact with. One such interaction, that has been frequently studied, is that occurring between the Gulf Stream and the DWBC at their cross-over point at Cape Hatteras. Models, with a wide range of complexity levels, have shown that the separation of the Gulf Stream from the coast and its subsequent path can change with changing transports of the DWBC (eg. Thompson and Schmitz (1989), Spall (1996a), Hurlburt and Hogan (2008)). All these investigators found that an increase in the DWBC can cause a southward displacement of the Gulf Stream axis. However, the amplitude of the response for a given change in the DWBC transport appeared to be a function of the vertical resolution of the model.

North-south displacements of the entire Gulf Stream axis are well documented (eg. Lee and Cornillon (1995) and Rossby and Benway (2000)), and like convective activity, appear to be correlated with changes in the NAO (Taylor and Stephens (1998)). Although other mechanisms have been proposed to explain this low frequency 
fluctuation in the Gulf Stream path (eg. Gangopadhyay et al. (1992) and Berloff et al. (2007)), the interaction with the DWBC appears an intriguing candidate since it provides a conduit for the changes at high latitudes to reach the subtropics. And as proposed by Joyce et al. (2000), the feedback from the Gulf Stream into the atmosphere, could close the loop and allow for oscillatory patterns. However, the relation between the Gulf Stream path and the MOC varies from model to model. Coetlogon et al. (2006) analyzed five different ocean circulation models, and found enhanced MOC to be associated with a northerly Gulf Stream position. The opposite was found by Zhang (2008), who attributed this difference to the coupling with the atmosphere. The recent work in Joyce and Zhang (2010), adding to Zhang's (2008) findings, presented some observational evidence supporting the connection between the strengthening of the MOC and a southerly Gulf Stream path. Those authors also pointed out that the resolution in most of the models discussed by Coetlogon et al. (2006) was not sufficient to properly resolve the Gulf Stream recirculations, thus they would likely miss interactions between the DWBC and the Gulf Stream.

In this emerging picture of the variability in the North Atlantic circulation, a number of questions are still unresolved. The particular ones that are the goal of this thesis can be summarized as follows: (1) is the DWBC an active pathway for the export of NADW?, (2) does the circulation in the DWBC respond to changes in the NADW formation rates and properties?, (3) what is the relative contribution of the different constituents of the NADW to the circulation?, and (4) do DWBC changes at depth affect the upper part of the water column, and in particular, do these upper ocean waters interact with the Gulf Stream?. These questions are addressed in the following four chapters. The part of the analysis addressing the forth question was done first for practical reasons ${ }^{1}$, and so it is presented first, in Chapter 2 . The remaining questions are addressed in the subsequent chapters.

\footnotetext{
${ }^{1}$ The Line $\mathrm{W}$ record, used in Chapters 3 and 4, was not complete until 2009.
} 


\subsection{Thesis outline}

Chapter 2 describes the surface variability in the western North Atlantic, including both the Gulf Stream and the Slope Water. A number of altimetry-derived products are used to characterized longer term changes in the latitude of the Gulf Stream and the circulation in the Slope Water. In combination with Sea Surface Temperature (SST) data, the Slope Water variability is described as the joint effect of changes in the hydrographic properties of waters that originate in the Labrador Sea and are brought into the region by the different elements of the circulation, and changes in the circulation itself. The chapter concludes with the analysis of a collection of conductivity/temperature/pressure (CTD) and direct velocity measurements on the continental slope at $69^{\circ} \mathrm{W}$ that link the variability seen at the surface in the 15 plus years of satellite data with changes occurring at depth.

In Chapter 3 we explore changes in the intermediate waters in the DWBC from 5.5 years of Moored Profiler data obtained above the continental slope southeast of Cape Cod during the period of 2002-2008. The last 4 years of the record were collected as part of the Line W moored array, which is later discussed in Chapter 4. The focus of the analysis in Chapter 3 is the water mass structure, with particular attention to changes occurring in the LSW. Using potential vorticity as a tracer for recently ventilated water, we explore the relationships between changes in the temperature, salinity and velocity fields and the ventilation process. The high temporal resolution of the moored time series allows us to calculate spreading rates for the different varieties of LSW that are then linked to changes in the circulation itself. Some final remarks on the fate of these waters downstream from Line $\mathrm{W}$ are made based on a series of repeat hydrographic sections extending all the way into the Gulf Stream.

The full Line W moored array data, spanning the period May 2004 to April 2008, is analyzed in Chapter 4 . The first section in the chapter introduces the data set and explains how the collection of measurements, with different spatial and temporal resolution, are combined to produce daily sections of temperature, salinity and 
horizontal velocity. An index indicating when the array was affected by Gulf Stream rings and meanders, that makes no use of additional data, is developed to separate this external source of variability in the array data from signals that are associated with the DWBC. The variability of the DWBC in the ring/meander-free time series, is characterized in terms of the joint variability of temperature, salinity, potential vorticity and horizontal velocity. Using this joint mode of variability, changes in the transport of the LSW and OW (the part that is resolved by the array) are explored.

In Chapter 5, we used the PV-invertibility principle to investigate the relative contribution of the observed changes in the different water masses to the observed changes in the circulation described in Chapter 4. A simplified version of the mean density distribution in the Slope Water/DWBC is combined with highly idealized PV anomalies with scalings inferred from the observations. Positive and negative PV anomalies at the levels of the uLSW, dLSW and OW are inverted separately first, and then combined to reproduce the observed conditions characterizing the early and later parts of the Line $\mathrm{W}$ record.

Finally, in Chapter 6 we summarize the most notable findings of this thesis, and relate them to the existing, but still developing, picture of the variability in the North Atlantic climate system, with special emphasis on those points where our actual knowledge presents some inconsistencies. 


\section{Chapter 2}

\section{Surface variability in the Slope Water and its relation to the Gulf}

\section{Stream path}

*The contents of this chapter are in part based on the paper "Variability in the Slope Water and its relation to the Gulf Stream path" by B. Peña-Molino and T. M. Joyce, Geophysical Research Letters, vol. 35, L03606, doi:10.1029/2007GL032183, 2008. The original time series in the paper have been extended, and new analysis added.

\section{$2.1 \quad$ Introduction}

A large fraction of the flow variability in the area of the Gulf Stream is related to fluctuations in the Gulf Stream path. Its transport and velocity structure appear quite stable over time (Rossby and Gottlieb (1998)). Fluctuations in the Gulf Stream position can be characterized by two modes (Lee and Cornillon (1995)): meanders, with typical length scales of ca. 100-400 km and periods ranging from 2 to 60 days, and wholesale latitude shifts that extend $1500 \mathrm{~km}$ into the basin and with time scales ranging from several months to several years. The cause of this latter low frequency fluctuation in the Gulf Stream path has been the subject of numerous studies. The theory for the steady circulation of the ocean predicts that the separation point of the Gulf Stream and its subsequent path is governed by the zero wind stress curl (Pedlosky 
(1979)). However, there is evidence that the zero wind curl line lies north of the separation point, and that the direct effect of wind stress changes alone cannot explain the variations in the Gulf Stream path (Zhang and Vallis (2007)). Nevertheless, it is generally accepted that these fluctuations in the Gulf Stream position are ultimately driven by the atmosphere. Typically, periods of low NAO are associated with a southerly Gulf Stream while during high NAO periods, the Gulf Stream is found north of its mean position (eg. Joyce et al. (2000), Taylor and Stephens (1989), Kelly et al. (1996) and Frankignoul et al. (2001)). These analyses of the relationship between the NAO and the Gulf Stream find that changes in the NAO lead changes in the Gulf Stream by periods ranging from 1-3 years. Several mechanisms have been proposed to explain this lag. Gangopadhyay et al. (1992) attributed the lag to the time required for westward propagating baroclinic Rossby waves to cross the Atlantic. A different mechanism for the relationship between atmospheric forcing and the latitude of the Gulf Stream was proposed by Joyce et al. (2000). They hypothesized that the strong atmospheric forcing at high latitudes led to enhanced dense water formation in the Labrador Sea, which changed the Gulf Stream position when the signal carried by the Deep Western Boundary Current (DWBC) reached the Gulf Stream cross-over point at Cape Hatteras.

The potential for the DWBC to affect the latitude of the Gulf Stream has been extensively studied in the modeling community. Motivated originally, at least in part, by an unrealistic overshoot of the Gulf Stream as it separates from the coast that is observed in a wide range of models, a series of experiments were carried out to understand the effect of the DWBC in the separation of the Gulf Stream and the latitude of the subsequent path. In the initial work by Thompson and Schmitz (1989), the mechanism for the interaction between the DWBC and the Gulf Stream at the cross-over relied on the ability of the across-isobath component of the flow in the upper part of the DWBC to effectively advect the thermocline (in the alongisobath direction at the cross-over). Thus the stronger the transport of the DWBC, the further south the Gulf Stream was found. Changes of some 40Sv were required 
to induce an overall shift in the Gulf Stream axis of $100 \mathrm{~km}$. This same mechanism has been later invoked by several other authors using different degree of complexity models (eg. Ezer and Mellor (1992), Spall (1996a) and Hurlburt and Hogan (2008)). Alternatively, in Zhang and Vallis (2007) the bottom vortex stretching associated with the downslope motion of the DWBC, both at the Grand Banks and Cape Hatteras, and the consequent formation of the Northern Recirculation Gyre, were found to be responsible for the changes in the position of the Gulf Stream.

In the present study we provide observational evidence of the relation between the circulation in the Slope Water, and the DWBC as one of its components, and the variability of the Gulf Stream path. To do so we will use satellite altimetry and Sea Surface Temperature (SST) in the western North Atlantic to characterize the different modes of variability both in the Gulf Stream and the Slope Water. The results from the surface analysis will be then linked to the circulation at depth by means of a series of repeat hydrographic sections along the continental slope at $69 \mathrm{~W}$. Finally, some remarks on the origin of the variability seen at the Slope Water will be made.

\subsection{Data}

\subsubsection{Remote sensing}

Monthly SST anomalies for 1993 to 2008 were obtained from the Reynolds data set (see Reynolds et al. (2006) for details), a combination of Advanced Very High Resolution Radiometer (AVHRR) and in situ data when available. The spatial resolution of the data is $1 / 4^{\circ} \times 1 / 4^{\circ}$. This product is relatively smooth, so features like warm core rings and meanders are typically not resolved. At each grid point, the time averaged and seasonal cycle were removed from the data yielding time series of SST anomalies.

Altimetric data for the same time period from the satellites TOPEX-Poseidon and Jason were obtained from AVISO (http://www.aviso.oceanobs.com/duacs), both in the form of Sea Surface Height (SSH) anomalies, and surface geostrophic velocities. 
The SSH data are corrected for atmospheric effects, surface conditions and other contributions such as tides and inverse barometer effect, and the long term mean removed. Along track data are provided every 10 days, and gridded velocities weekly. For the purpose of this study, the characterization of Slope Water circulation anomalies is based on the along-track SSH anomalies. The accuracy of the gridded velocity product within the Slope Water is not sufficient to resolve the circulation. The amplitude of the variability associated with the Gulf Stream is, on the other hand, large enough to be captured by the gridded velocities, so a combination of along-track and gridded velocities were used. It is important to emphasize that in both cases we will only be considering anomalies, since the resolution of the available mean dynamic topography is much lower than that of the anomaly fields (Rio and Hernandez (2004)).

Surface geostrophic velocities in the Slope Water were obtained from the alongtrack gradient of the SSH anomalies. These velocities are by definition normal to the track, which is fortuitously in the along-isobath direction within the Slope. In our adopted coordinate system, positive velocities are to the northeast (downstream in the mean Gulf Stream) and negative to the southwest (also downstream for mean Slope Water flow). The velocities normal to the tracks are projected onto the $1 / 4^{\circ} \times 1 / 4^{\circ}$ SST grid by linear interpolation between adjacent altimeter tracks.

\subsubsection{Hydrographic data}

The vertical structure of the Slope Water variability was investigated using a total of 13 hydrographic surveys. The stations were collected along a transect spanning the continental slope approximately at $69^{\circ} \mathrm{W}$ that is coincident with TOPEX-Poseidon altimeter track 126. The data used here consist of CTD and direct velocity measurements, between 1994 and 2008 (Table 2.1). For the period 2004 to 2008 the surveys were carried out as part of the Line W program (http://www.whoi.edu/science/PO/linew/index.htm). These sections were typically occupied in mid-spring and mid-fall.

To minimize the direct effect of the Gulf Stream, stations offshore from the $4500 \mathrm{~m}$ isobath were neglected. The sections were interpolated into a regular $20 \mathrm{~km} \mathrm{x} 50 \mathrm{~m} \mathrm{x-z}$ 
Table 2.1: Hydrographic surveys.

\begin{tabular}{cccc}
\hline \hline $\begin{array}{r}\text { Cruise No. } \\
\text { (SPRING) }\end{array}$ & Date & $\begin{array}{c}\text { Cruise No. } \\
\text { (FALL) }\end{array}$ & Date \\
\hline- & - & En257 & Nov 1994 \\
Oc269 & Jun 1995 & - & - \\
En256 & Aug 1996 & - & - \\
- & - & Kn173_2 & Nov 2003 \\
Oc401 & Apr 2004 & Kz124 & Sep 2004 \\
Oc411 & Apr 2005 & Oc417 & Oct 2005 \\
Oc421 & Apr 2006 & Oc432 & Oct 2006 \\
Oc436 & Apr 2007 & En440 & Oct 2007 \\
Oc446 & May 2008 & - & - \\
\hline
\end{tabular}

grid, and a least-squares estimate of the seasonal cycle was constructed and removed from the CTD data. No significant seasonality was found in the velocity data.

Additional shipboard ADCP data along a transect between New Jersey and Bermuda obtained from the Oleander Project (see http://www.po.gso.uri.edu/rafos/research/ole for details) for the period 1994 to 2007 were used to characterize the mean surface velocity field across the Slope Water and Gulf Stream. Individual $M / V$ Oleander transects were projected into a common line centered at $70^{\circ} \mathrm{W}$ (Figure 2-1), the projected velocities rotated into the direction normal to the altimeter tracks and averaged providing a description of the Eulerian mean circulation.

\subsection{The surface signal. Part I: SST and the Gulf Stream PC1 lag-correlation}

The synoptic velocity of the Gulf Stream at the surface may be represented as an asymmetric Gaussian jet with a sharper cyclonic side to the north and a broader anticyclonic side (eg. Rossby and Gottlieb (1998) and Johns et al. (1995)). As the core of the jet shifts in the cross-stream direction, both due to meandering and low frequency latitude shifts, the resulting Eulerian mean has a similar structure but 

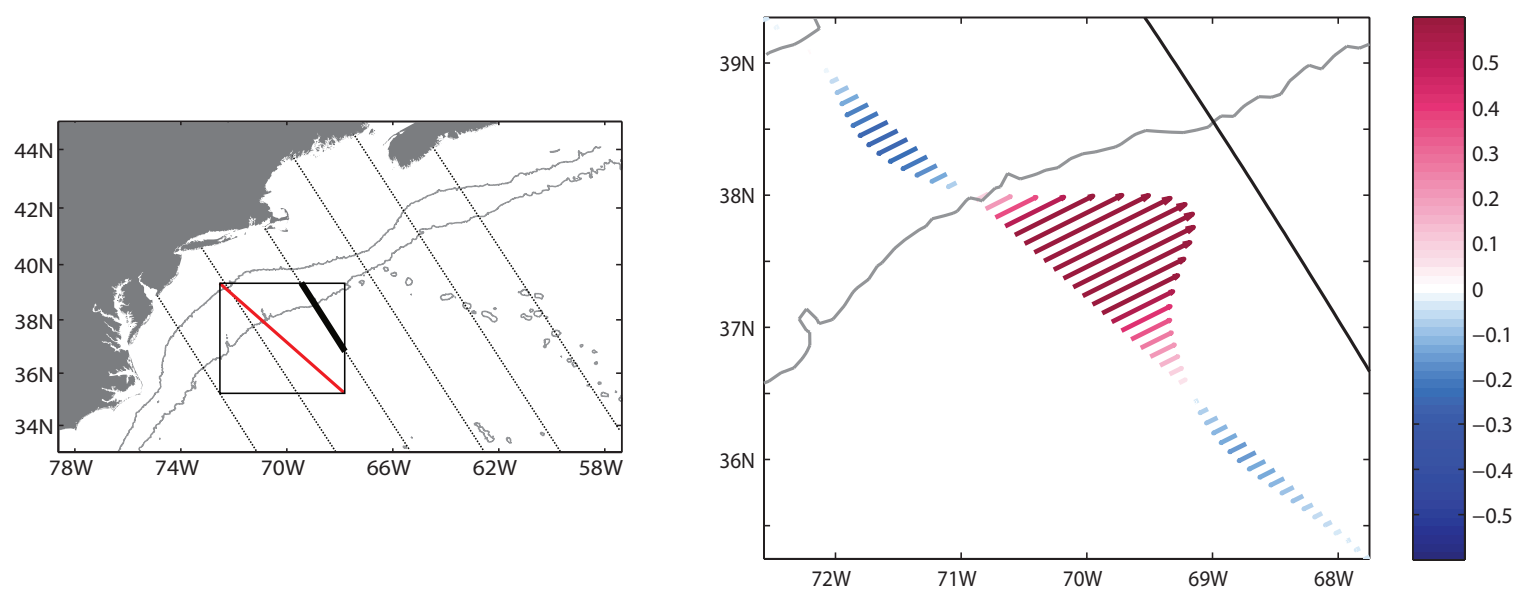

Figure 2-1: The map on the left shows the location of the $M / V$ Oleander track in red. The altimeter tracks are represented by the dashed black lines. The thick solid line shows the location of Line $\mathrm{W}$ with respect to the Oleander track. The panel on the right shows a blow-out of the box in the map on the left showing mean surface velocity $(\mathrm{m} / \mathrm{s})$ along the Oleander track. The gray contours showing the 1000 and $3500 \mathrm{~m}$ isobaths.

with smaller peak amplitude and wider distribution. At the core of the Gulf Stream the peak Eulerian mean velocity is approximately $1 \mathrm{~m} / \mathrm{s}$, about half of the streamcoordinates mean and synoptic Gulf Stream (2m/s; Johns et al. (1995)).

In order to characterize the variability of the Gulf Stream path in the absence of an accurate SSH-derived mean field we proceeded in two steps. First we estimated the axis position of the Gulf Stream in each altimeter pass by searching for the maximum gradient in the along track SSH anomalies (the maximum velocity anomaly) as described in Kelly and Gille (1990). Because the signature of the Gulf Stream becomes more diffuse east of $60^{\circ} \mathrm{W}$, only 6 tracks spanning the region between $74^{\circ} \mathrm{W}$ and $62^{\circ} \mathrm{W}$ were employed. After the time series of synoptic Gulf Stream axis position at all six locations were obtained, the mean was calculated. Next, the gridded AVISO surface geostrophic velocity anomalies for the western North Atlantic sector were rotated into the mean direction of the Gulf Stream. We subsequently used EOF analysis of the rotated velocities to explore the dominant modes of variability. The first and second modes of the monthly velocity anomalies, figure 2-2, explained respectively $10 \%$ and $6 \%$ of the velocity variance in the vicinity of the mean Gulf Stream (100km to the 
north and $100 \mathrm{~km}$ south of the mean path), where the amplitude of the mode is large enough for the signal to be significant. Over the entire western North Atlantic sector, including regions away from the Gulf Stream were the velocity is noisy, the first and second modes only explained $4 \%$ and $3 \%$ of the variance respectively.

The first mode may be characterized as an in phase north-south shift of the entire Gulf Stream axis. It is zero around the mean position of the Gulf Stream axis and has opposite sign on either side of the mean axis. When the mode is positive north of the mean path, it indicates the Gulf Stream core is displaced northward. Similarly when the mode is positive south of the mean, it indicates the Gulf Stream occupies a southerly position. The amplitude of these displacements ranges between 50 and $100 \mathrm{~km}$. The second mode is centered around the mean Gulf Stream axis west of $62^{\circ} \mathrm{W}$, indicating a north-south shift as well, but in this case with a node at $70^{\circ} \mathrm{W}$. This structure implies that when the Gulf Stream is north of its mean position between Cape Hatteras and $70^{\circ} \mathrm{W}$, it is south of its mean position east of $70^{\circ} \mathrm{W}$. The two phases of this mode represent changes in the tilt of the Gulf Stream axis. When the mode is positive (as shown in figure 2-2) the path is more linear, with a stronger meridional component over the entire domain. When the mode is negative (opposite phase to figure 2-2) the Gulf Stream path first overshoots north from the separation point, and then follows a more zonal path east of $70^{\circ} \mathrm{W}$.

The time behavior of the first principal component, GS-PC1, is a low frequency oscillation with alternating periods of negative (southerly Gulf Stream) and positive (northerly Gulf Stream). The frequency spectrum of the first principal component shows energy at 3-year period (figure 2-3). The fluctuations in the second mode, GS-PC2, exhibit energy at shorter periods. The second mode's spectrum shows a high-energy band around 250 days, and secondary peaks around 100 days and 2 years. The spectrum of the DWBC transport calculated from the Line W array for the period 2004-2008 also shows a peak near 100 days (personal communication T. Joyce), suggesting this mode could be associated with fluctuations in the DWBC flow. Both principal components show hints at times of a seasonal cycle (eg. between 

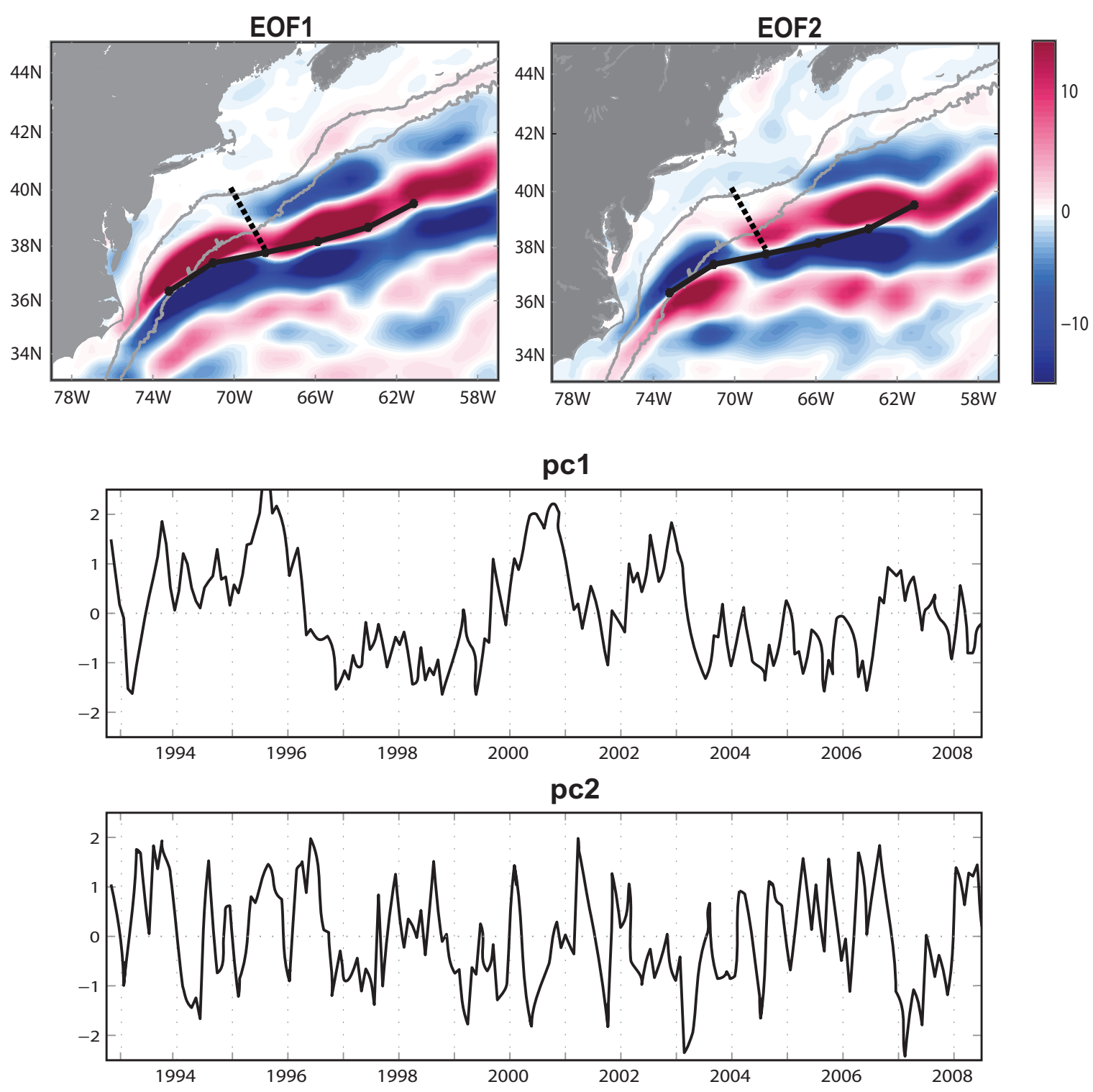

Figure 2-2: First and second EOF of the downstream (rotated into the mean direction of the Gulf Stream) velocity anomalies. Spatial patterns are shown in the top panels and corresponding principal components in the bottom panels. The units, $\mathrm{cm} / \mathrm{s}$, are contained in the spatial pattern, while the pc's dimensionless (normalized to have standard deviation of 1). The gray contours in the maps correspond to the 1000 and $3500 \mathrm{~m}$ isobaths, the solid black line is the mean position of the Gulf Stream estimated from the along track SSH anomalies, and the dashed black line is the location of the Line $\mathrm{W}$ observations. 

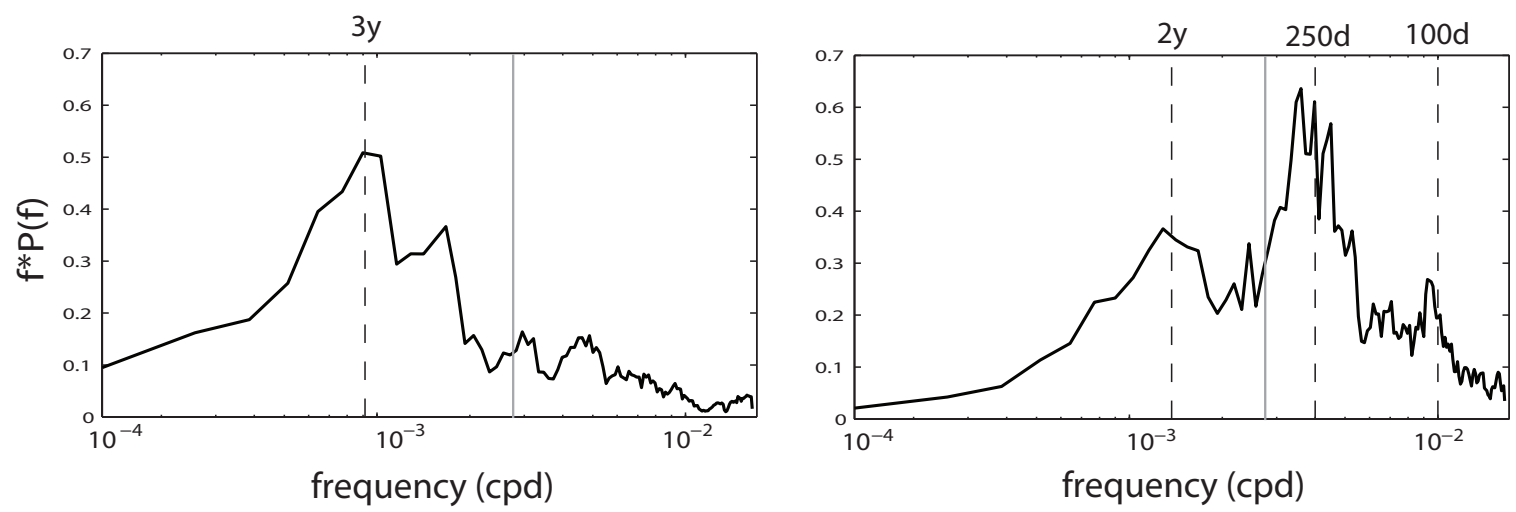

Figure 2-3: Multitaper spectrum of PC1 on the left and $\mathrm{PC} 2$ on the right. The units in both axis are cycles per day since the principal components are normalized (dimensionless).

2006-2007 in PC1 or 1995-1996 in PC2), corresponding to a more southerly Gulf Stream in the western part of the domain in the summer and more northerly in the winter, similar to what is found by others (eg. Lee and Cornillon (1995) and Kelly and Gille (1990)). The spectra, however, do not show any distinct peak at the annual frequency.

Lee and Cornillon (1995) found that the intensity of meandering activity in the Gulf Stream fluctuates at periods of approximately 9 months as well as 2 years (the characteristic periods of the meanders themselves being shorter), similar to the energetic bands shown in figure 2-3 for PC2. In their work, Lee and Cornillon (1995) found that the correlation between meandering activity and north-south shifts of the Gulf Stream path (here represented by the first Gulf Stream mode), was weak. The stability properties of the Gulf Stream, and hence the meandering activity, are more likely to be altered with changes in the meridional tilt of the current, like those represented by the second mode, rather than by the in-phase lateral shifts of mode 1 .

Rossby (1999) and Rossby and Benway (2000) found that fluctuations in the Gulf Stream position correlate with changes in the surface temperature and salinity changes in the Slope-Shelf region. Before the Gulf Stream shifts north, the Slope-Shelf surface temperature rises. These authors proposed that the time varying inflow from the Labrador Current that was responsible for these fluctuations. We investigated 
the relation between the Gulf Stream path and the surface temperatures in the Slope by computing lag-correlations between the GS PC1 and the Slope SST anomalies, figure $2-4$.

Large correlation values were found north of the Gulf Stream in a well defined pattern centered between the 1000 and $3500 \mathrm{~m}$ isobaths. The positive correlation, indicating that warming (cooling) is associated with northward (southward) shifts of the Gulf Stream, is significant (at the $90 \%$ significance level) for lags ranging from -12 to +2 months. The negative sense of the lag means that SST changes precede shifts of the GS path, as was found by other authors (Rossby (1999) and Rossby and Benway (2000)). As the lag advances, the patch of high correlation gradually spreads from the eastern side of the domain towards the Gulf Stream/DWBC cross-over point. At zero lag the correlation is significant only in the vicinity of the separation point.

Taking a deeper look at the westward propagation of SST anomalies in the first mode, we find this propagation is mostly associated with low frequency variability in the data. If we average the Slope SST anomalies at each longitude and compute the coherence between the averaged SST anomalies and the GS PC1 (figure 2-5) we find that there is a band at the low frequency limit, periods longer than 2-3 years $($ freq $<0.04-0.03$ cycles per month, cpm), where the coherence is significant.

The coherence phase within the significant band changes approximately linearly with frequency; the slope of this linear relation provides an estimate of the lag. The rate of change of the lag with distance, given by the longitude axis in figure 2-5, is the speed of propagation of the SST features. For frequencies $<0.04 \mathrm{cpm}$ we find that the temperature anomalies that correlate with the Gulf Stream path are moving zonally with a speed of $-9 \pm 3 \mathrm{~cm} / \mathrm{s}$ (negative speeds meaning westward). This estimate represents both a spatial mean over the entire Slope Water region, and a temporal mean for the whole length of the record. It is comparable to the $-13 \mathrm{~cm} / \mathrm{s}$ mean downstream velocity from the Oleander section between the $1000 \mathrm{~m}$ and $3500 \mathrm{~m}$ isobaths, suggesting simple advection of SST anomalies by the mean Slope flow.

The GS-PC2 has a very different relation with the Slope temperature field. The 

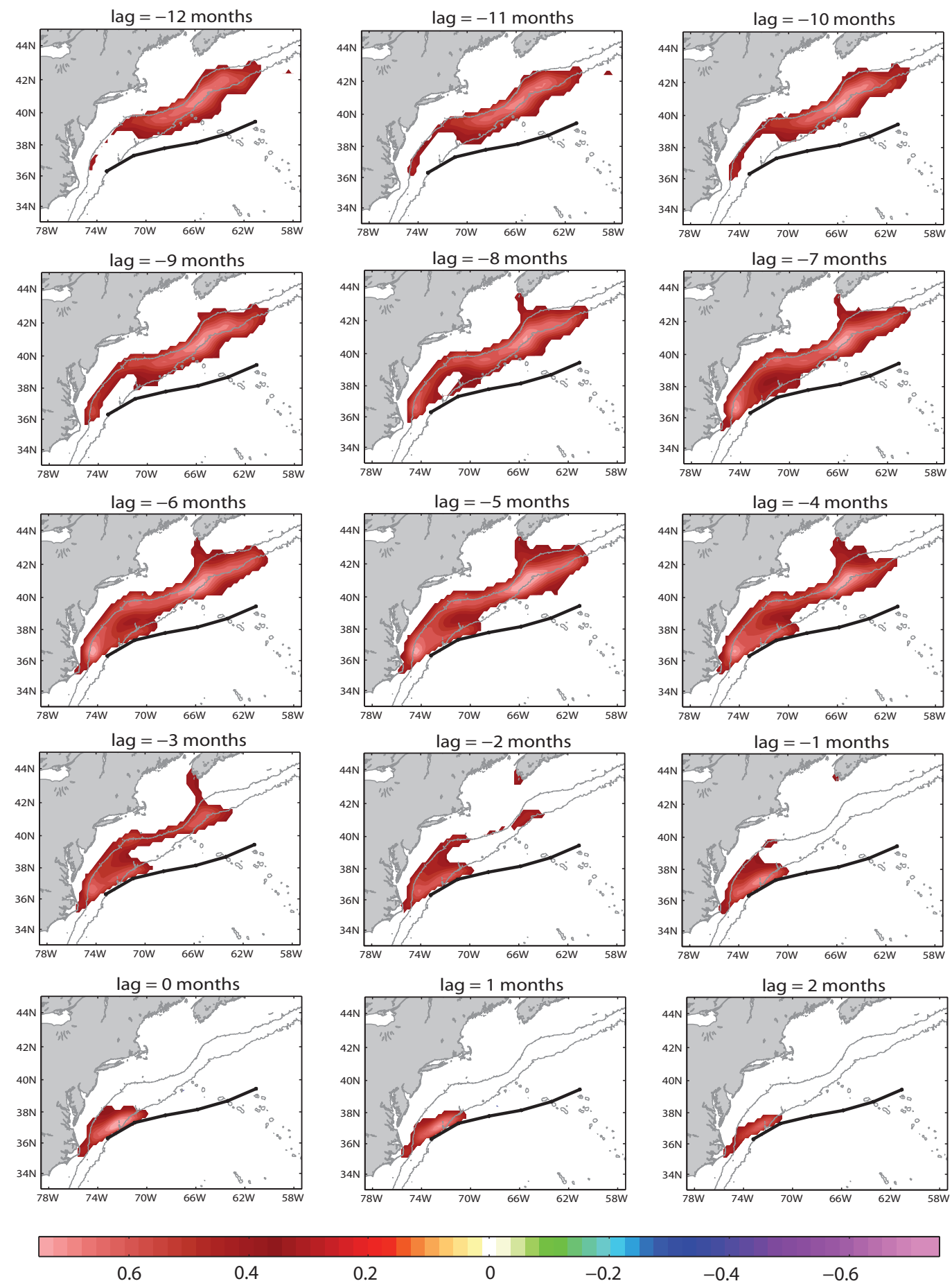

Figure 2-4: Cross-correlation between the SST anomalies and Gulf Stream path index for lag values ranging from -1 year (SST leading) to +2 months (SST following). Values below the $90 \%$ significance level have been masked out. No significant correlations were found after the 2 month lag. The black line is the mean position of the Gulf Stream inferred from altimetry. Grey contours represent the 1000 and $3500 \mathrm{~m}$ isobaths. 

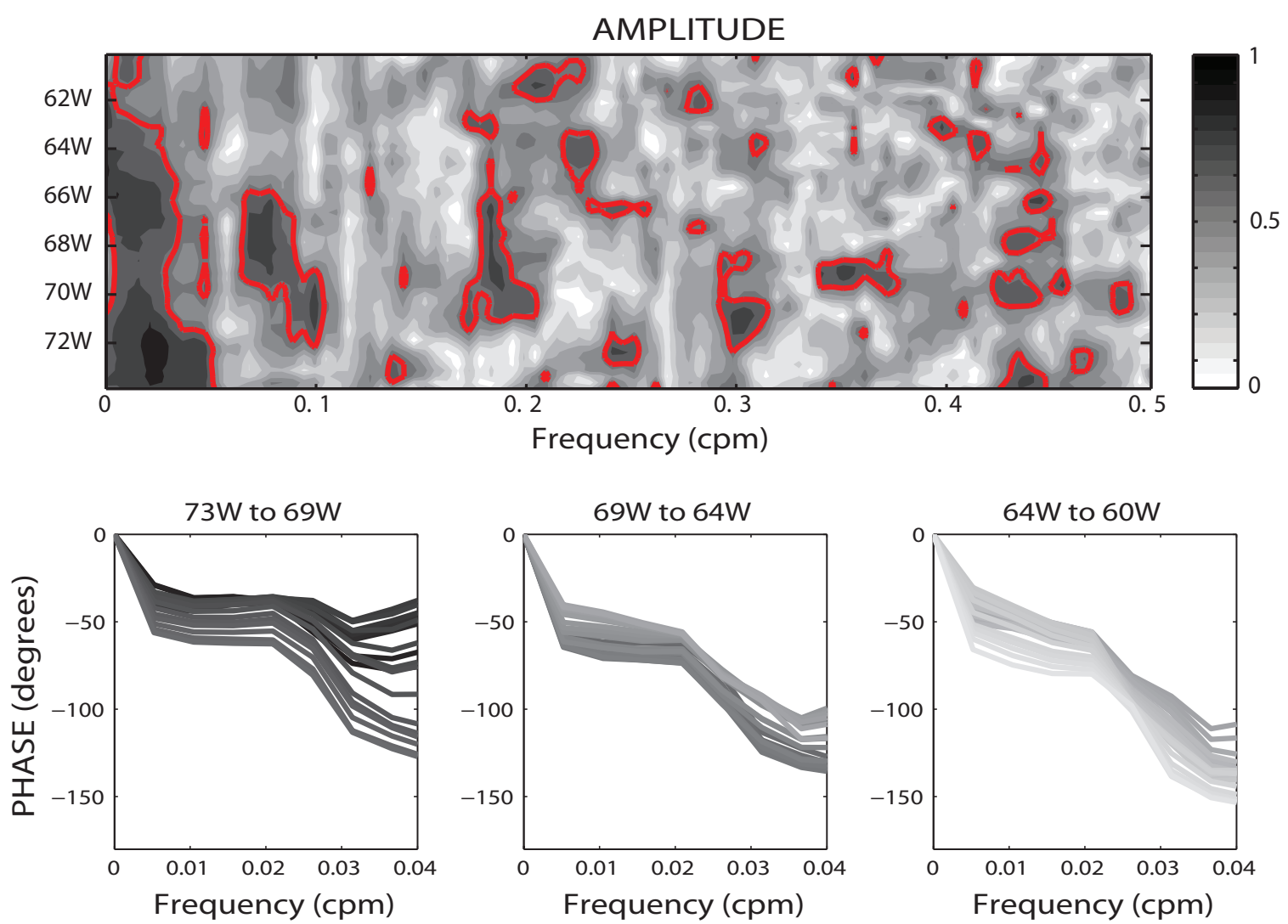

Figure 2-5: Upper panel shows coherence amplitude, ranging from 0 to 1, of the SST anomalies and the Gulf Stream position as a function of longitude and frequency, the latter in cycles per month. Red contours denote the $90 \%$ signifcance level. The lower panels show coherence phase versus frequency (from -180 to 0 in degrees) for the frequency band $0-0.04 \mathrm{cpm}$ for all longitudes between $73^{\circ} \mathrm{W}$ and $69^{\circ} \mathrm{W}$ on the left, $69^{\circ} \mathrm{W}$ to $64^{\circ} \mathrm{W}$ on the middle, and $64^{\circ} \mathrm{W}$ to $60^{\circ} \mathrm{W}$ on the right. Within each panel the color grading (from light to dark) indicates the east to west progression.

largest values of the correlation, and only significant feature at the $90 \%$ confidence level, occurs at zero lag (figure 2-6). The pattern consists of alternating positive and negative correlation patches north of the Gulf Stream. Along the mean Gulf Stream path, the mode is consistent with what is observed in the velocity field, and SST changes can be explained by the north-south advection of the temperature gradient due to displacements in the Gulf Stream, $v \partial T / \partial y$ in our rotated reference frame. The cross-stream temperature gradient, $\partial T / \partial y$, has the same sign everywhere to the north of the Stream's core, but the north-south velocity, $v$, is negative west of EOF2's node at $70^{\circ} \mathrm{W}$ when GS-PC2 $>0$, and it is positive east of the node, explaining the 


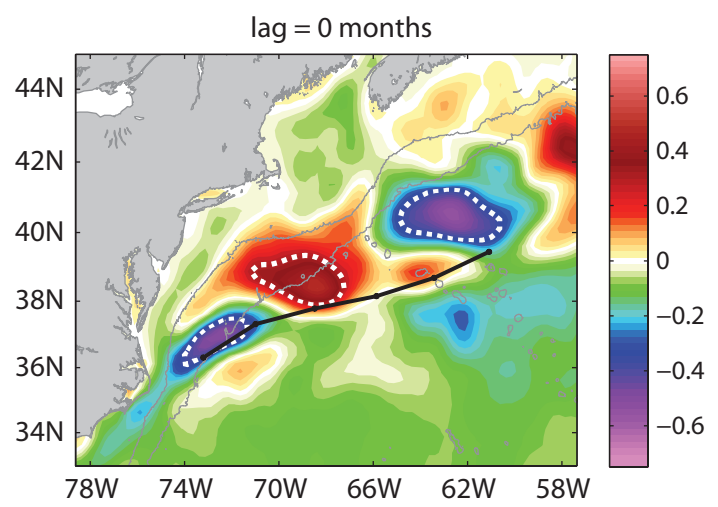

Figure 2-6: Cross-correlation between PC2 of the Gulf Stream position and SST anomalies at zero lag. The white dashed lines marked the $90 \%$ significance level. The gray contours are the 1000 and $3500 \mathrm{~m}$ isobaths and the black line is the mean position of the Gulf Stream.

sense of the correlation. East of the New England Seamount chain, the reversal in the sense of the correlation can also be explained in terms of advection. In this region, however, the EOF2 is not centered around the mean Gulf Stream path, instead the zero contour line that separates the positive and the negative vorticity cores is south of the mean axis, and the sense of the north-south advection of temperature is the same as in the region east of the separation point.

An alternative way to explore the contribution from advection to the local changes in sea surface temperature and estimate the mean along-isobath velocity in the Slope Water that is consistent with the SST changes, is from a vertically integrated heat budget for the mixed layer. Temperature, velocities, mixed-layer thickness and heat fluxes are separated into a mean and a perturbation. Subtracting the mean balance:

$$
\left\langle\bar{u} \frac{\partial \bar{T}}{\partial x}\right\rangle=\left\langle\frac{\bar{Q}}{\rho C_{p} \bar{h}}\right\rangle
$$


we are left with the following perturbation equation:

$$
\begin{array}{r}
\left\langle\frac{\partial T^{\prime}}{\partial t}\right\rangle+\left\langle\bar{u} \frac{\partial T^{\prime}}{\partial x}\right\rangle+\left\langle u^{\prime} \frac{\partial \bar{T}}{\partial x}\right\rangle+\left\langle u^{\prime} \frac{\partial T^{\prime}}{\partial x}\right\rangle+\left\langle\bar{v} \frac{\partial T^{\prime}}{\partial y}\right\rangle+\left\langle v^{\prime} \frac{\partial \bar{T}}{\partial y}\right\rangle+\left\langle v^{\prime} \frac{\partial T^{\prime}}{\partial y}\right\rangle \\
=\left\langle\frac{Q^{\prime}}{\rho C_{p} \bar{h}}\right\rangle+\left\langle\frac{\bar{Q}}{\rho C_{p} \bar{h}} \frac{h^{\prime}}{\bar{h}}\right\rangle
\end{array}
$$

where the $x$ and $u$ are the along-isobath distance and velocity, $y$ and $v$ are the acrossisobath distance and velocity, $h$ is the mixed layer depth, and the remaining variables have their usual meaning ${ }^{1}$. The angle brackets denote spatial averages within the Slope.

In order to estimate the mean along-isobath velocity in the Slope, $\bar{u}$, from the heat budget perturbation equation we calculated perturbation velocities the altimeter data: the along-isobath component, $u^{\prime}$, from geostrophy, and the across-isobath, $v^{\prime}$, as due to the north-south fluctuations of the Gulf Stream position. In the absence of an estimate of the variations in the mixed-layer depth, we assumed these are small compared to the mean mixed-layer depth so that last term in the equation can be ignored,. It is also assumed that $\bar{u}$ and $\partial T^{\prime} / \partial x$ are spatially uncorrelated, $\left\langle\bar{u} \partial T^{\prime} / \partial x\right\rangle=\langle\bar{u}\rangle\left\langle\partial T^{\prime} / \partial x\right\rangle$, and that $\bar{v}=0$. Using heat flux anomalies from Yu et al. (2004) the mean along-isobath velocity was finally estimated as a free parameter in the least-squares fit of the perturbation equation with the variables just described. The resulting mean flow for the Slope Water was $-6 \mathrm{~cm} / \mathrm{sec}$ to the $\mathrm{SW}$. This estimate of the mean velocity in the Slope was found to be robust. Its value, as we gradually increase the number of advection terms used in the fit, from 2 to 5 , remained stable. We also found that the percentage of the local rate of temperature change explained by the different advection terms vary within the Slope, with $v^{\prime} \partial \bar{T} / \partial y$ and $\bar{U} \partial T^{\prime} / \partial x$ being the largest contributors. The sum of all the contributions from all advection terms exceeded the contribution from the heat flux.

\footnotetext{
${ }^{1} T$ is the sea surface temperature, $\rho$ is density, $C_{p}$ is the specific heat and $Q$ is the heat flux.
} 


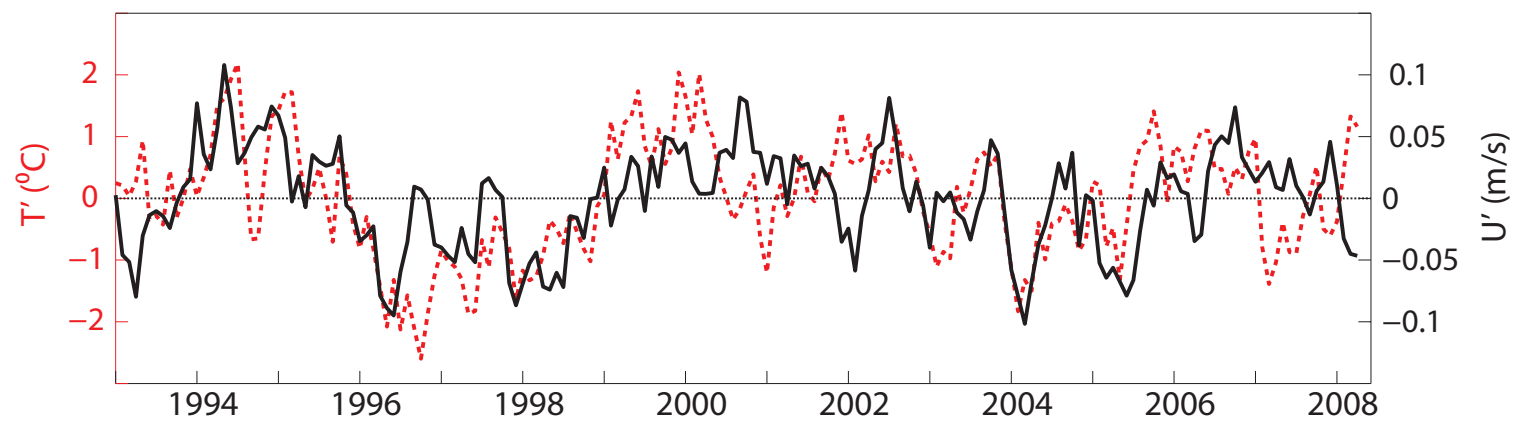

Figure 2-7: Ensemble of the temperature, in red, and surface geostrophic velocities, in black, in the Slope Water.

\subsection{The surface signal. Part II: modes of surface velocity and SST variability}

In the previous section we saw that westward propagating SST anomalies in the Slope Water precede lateral shifts of the Gulf Stream axis. These SST anomalies in the Slope can be due to the advection by the mean flow of water with changing temperature, the advection of water of with constant temperature by a changing flow, or a combination of both. The contribution of the mean flow is clear from the agreement between the propagation speed of the SST anomalies and the mean velocity estimated from the Oleander data. Petrie and Drinkwater (1993) showed that the variability in the temperature in the Scotian Shelf is mostly driven by changes in circulation. The same may be true in the Slope. To investigate the relation between changes in the circulation and the SST anomalies in the Slope we used surface geostrophic velocities calculated from the along-track altimeter data describe in section 2.2.1. We defined the slope as the region between the $1000 \mathrm{~m}$ and $3500 \mathrm{~m}$ isobaths where the SST/GS-PC1 correlation was significant. The ensemble mean of the surface geostrophic velocity anomalies, $\langle u \prime\rangle$, and surface temperature anomalies, $\left\langle T^{\prime}\right\rangle$, in figure 2-7, are correlated at the $95 \%$ confidence level. The correlation is positive, indicating that cooling (warming) is linked to stronger (weaker) southwestward (SW) flow.

However, $\langle u \prime\rangle$ and $\left\langle T^{\prime}\right\rangle$ do not provide any information about the spatial 
structure of the correlation and, as suggested by the differences in the longitude dependence of the lag-correlation between the GS-PC1 and the SST anomalies, this correlation may not be spatially homogeneous. To investigate the spatial variability of the relation between the SST anomalies and the surface geostrophic velocities, we calculate the joint EOF of both variables. In order to obtain a leading mode that is stable both in time and space, and well-separated from consecutive modes, the first three modes, explaining $25 \%$ of the variance, were rotated using VARIMAX rotation. The VARIMAX rotation maximizes the variance of the first mode and, in cases where the leading modes are not well-separated, helps extract more physically meaningful modes (von Storch and Zwiers (1999)). The first rotated mode (figure 2-8) shows cooling and strengthening of the southwestward flow everywhere east of $69^{\circ} \mathrm{W}$. West of $69^{\circ} \mathrm{W}$, the pattern is more complicated. Near the $3000 \mathrm{~m}$ isobath, between $73^{\circ} \mathrm{W}$ and $71^{\circ} \mathrm{W}$ a large negative anomaly in velocity is found. This is due to the direct influence of the Gulf Stream, and it represents a south shift of the Gulf Stream (north when $\mathrm{PC} 1<0)$. North of this feature, in the shallower water, the mode reverses sign, which implies a reduction of the SW flow. This reduction of the SW flow is most likely related to the westward recirculation north of the Gulf Stream (Johns et al. (1995)). When the Gulf Stream path shifts to the south, the westward flow associated with the recirculation is also displaced, leaving a positive velocity anomaly behind. The amplitude of this positive anomaly obtained in our EOF analysis is consistent with Johns et al's (1995) estimates of the Gulf Stream recirculation ( -3 to $-4 \mathrm{~cm} / \mathrm{s})$.

The corresponding temperature spatial pattern is negative (cooling) everywhere within the Slope, with a very homogeneous amplitude. There is no clear signal of the Gulf Stream, as was the case for the velocity mode (figure 2-8 left panel), perhaps due to the larger degree of smoothing in the SST data compared to the along-track SSH anomalies.

The leading principal component associated with this mode (bottom panel figure 2-8) shows alternating periods of large negative values and large positive values every 3 years or so. It has a very similar character to GS-PC1 in figure 2-2, and in fact the 

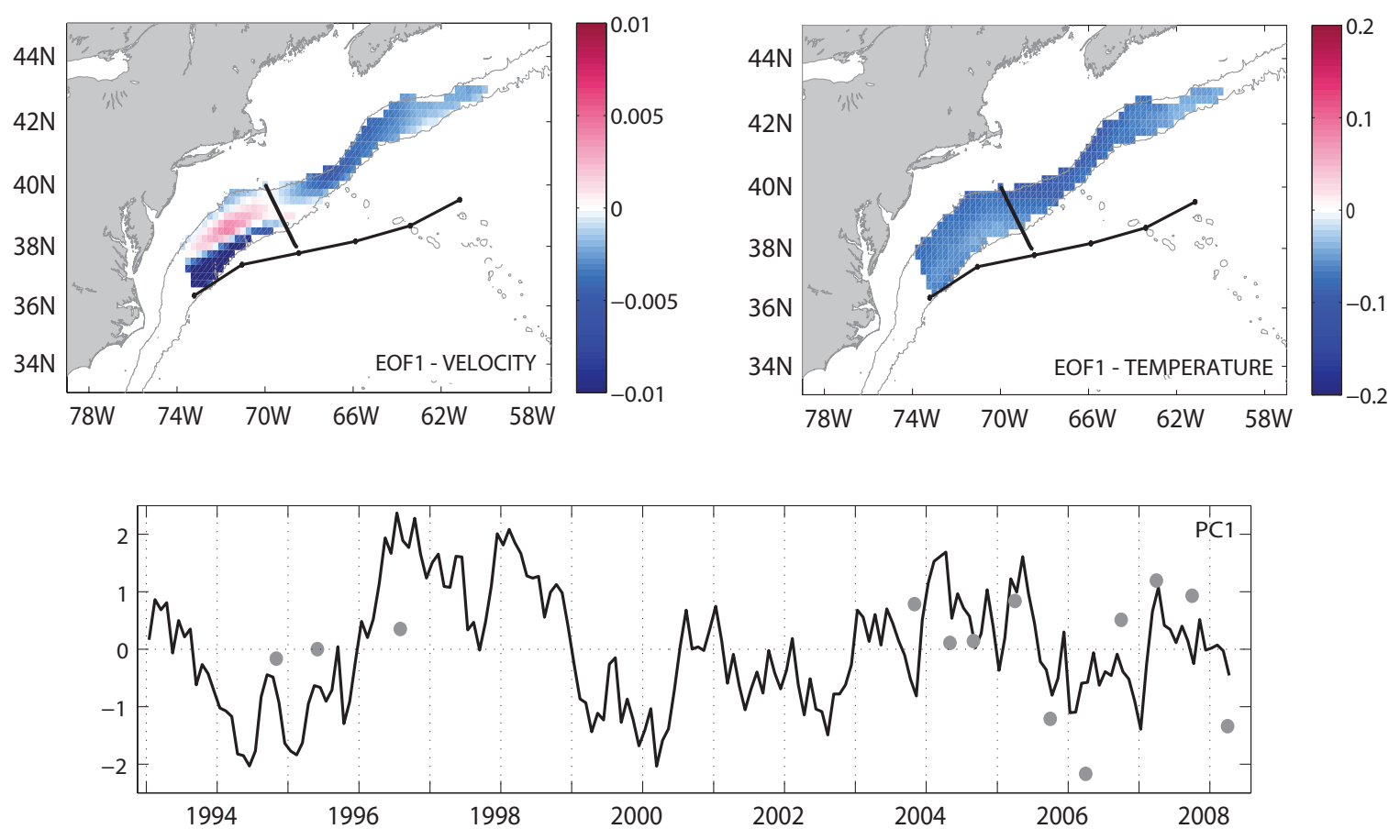

Figure 2-8: First rotated EOF of the surface geostrophic velocity, on the left, and SST anomalies, on the right. The black line near $69^{\circ} \mathrm{W}$ is the Line $\mathrm{W}$ transect. The thin line is the mean position of the Gulf Stream. The bottom panel shows the corresponding principal component, PC1, in black. The gray dots are the principal component of the leading EOF of the hydrographic data discussed in section 2.6.

time series are significantly correlated. The correlation is maximum when changes in the combined SST-velocity mode lead changes in the Gulf Stream path by 5-6 months (figure 2-9). This implies, once again, that preceding southward shifts of the Gulf Stream axis, the temperature north of the Gulf Stream cools and the mean southwestward flow within the Slope Water east of $69^{\circ} \mathrm{W}$ intensifies.

\subsection{The origin of the anomalies}

We have seen that SST anomalies observed in the western North Atlantic appear to be due to changes in the temperature some distance upstream. Can these anomalies be traced back to the northern North Atlantic? To answer this question, we computed the Complex EOF (CEOF) of the Reynolds SST data set for the western North 


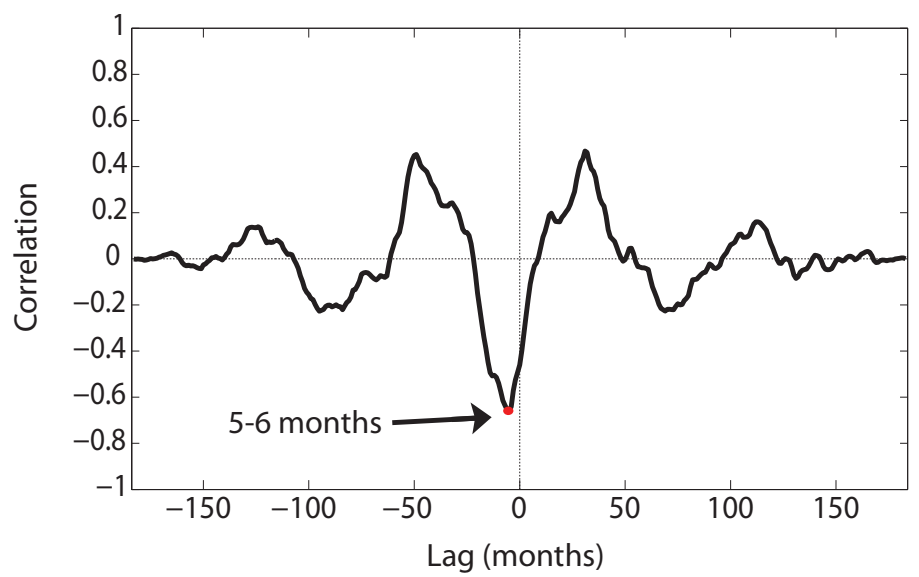

Figure 2-9: Cross-correlation between the leading principal component of the Gulf Stream surface geostrophic velocities, pc1 in figure 2-2, and the leading principal component of the joint EOF of the surface geostrophic velocity and SST anomalies in the Slope (bottom panel figure 2-8. The negative value of the lag indicates that changes in the Slope preceed changes in the Gulf Stream position.

Atlantic north of Cape Hatteras including the Labrador Sea. CEOF is a very useful tool for detecting propagating signals. The time evolution of the signal is represented as a complex time series, $Z(\mathbf{x}, t)$, which is constructed as the sum of the Hilbert transform of the data, $\hat{X}(\mathbf{x}, t)$ as the imaginary part, and the original time series $X(\mathbf{x}, t)$, as the real part (Horel (1984)). All this does is add a phase shift of +90 degrees to the signal while keeping its amplitude constant. This way the information about the time rate of change of the signal is introduced.

$$
Z(\mathbf{x}, t)=X(\mathbf{x}, t)+i \hat{X}(\mathbf{x}, t)
$$

One then proceeds with the decomposition of the new variable $Z(\mathbf{x}, t)$ in a similar fashion to regular EOFs. Now however, both the spatial patterns, $\tilde{S}(\mathbf{x})$, and the principal components, $\tilde{P}(t)$, have imaginary as well as real parts. The amplitude of the spatial pattern, $S(\mathbf{x})$, equivalent to regular EOFs, is obtained from the square of the complex spatial pattern, 

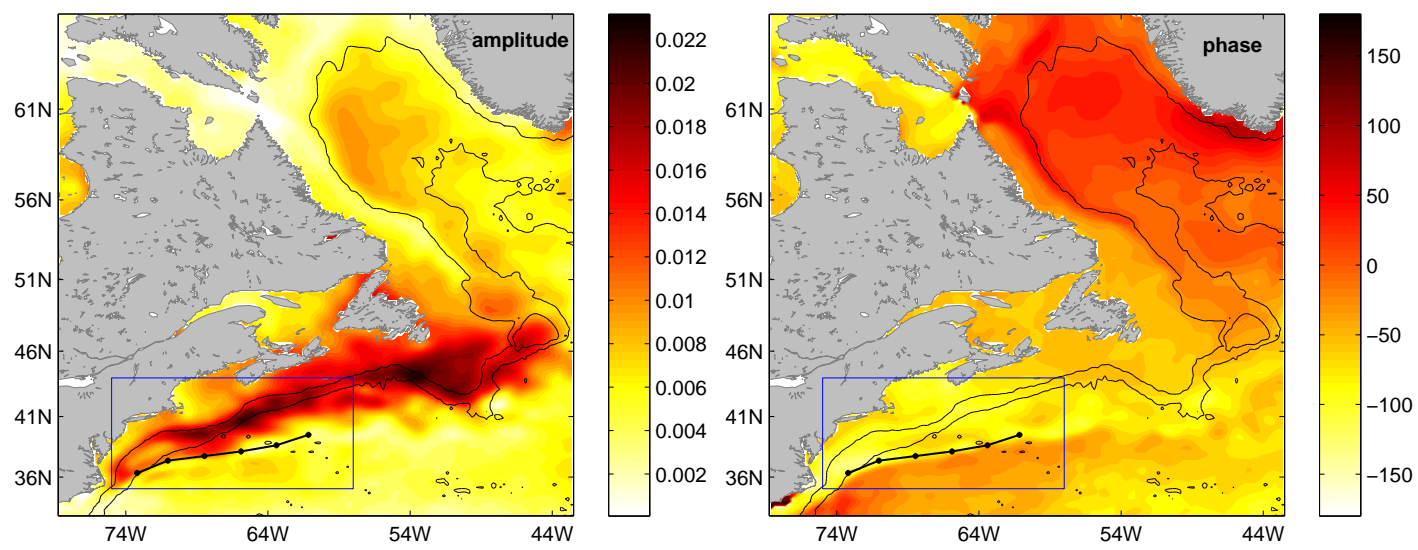

Figure 2-10: Spatial pattern amplitude, on the left, and phase, on the right, of the leading CEOF of SST anomalies. The black contours denote the 1000 and $3500 \mathrm{~m}$ isobaths. The region highlighted by the blue box is the location of the region used in sections REF and REF, and the black dotted line inside the mean position of the Gulf Stream estimated from altimetry.

$$
S(\mathbf{x})=|\tilde{S}(\mathbf{x})|
$$

The phase $\theta_{S}(\mathbf{x})$, having no equivalent in a regular EOF analysis, may be recovered from the ratio of imaginary and real parts

$$
\theta_{S}(\mathbf{x})=\arctan \frac{\operatorname{Im}(\tilde{S}(\mathbf{x}))}{\operatorname{Re}(\tilde{S}(\mathbf{x}))}
$$

Because the phase shift introduced by the Hilbert transform is positive, the phase of the spatial pattern tells us how a signal, with amplitude provided by $S(\mathbf{x})$, propagates from regions where the phase is positive (large) to regions where the phase is negative (small). The origin for the phase estimate is arbitrary.

The amplitude of the spatial pattern of the leading CEOF (figure 2-10) is maximum in the region north of the Gulf Stream between the $1000 \mathrm{~m}$ and $3500 \mathrm{~m}$ isobaths, beginning around Flemish Cap, $50^{\circ} \mathrm{N}$, and extending all the way to Cape Hatteras. 
Although the mode's amplitude in the central Labrador Sea is much smaller, about half that at mid latitudes, there is some indication of the signal being steered along the same isobaths. The spatial structure of the phase following the path of the high amplitude signal shows decreasing values from the western side of the central Labrador Sea south along the boundary to the Gulf Stream separation point, suggesting that SST anomalies originated in the Labrador Sea region.

What causes the signal amplification south of Flemish Cap is unclear at this point. One possible explanation is the interaction between the upper flow in the DWBC and the warm waters carried by the Gulf Stream/North Atlantic Current. The water occupying the upper layers in the Slope is known to be a mixture of waters originating at high latitudes and Gulf Stream waters (Csanady and Hamilton (1988)). At the tip of the Grand Banks, as well as the Gulf Stream separation point at Cape Hatteras, the southward flowing cold waters and northward flowing warm waters converge into a small region where lateral mixing might occur (Csanady and Hamilton (1988)). Changes in the amount of warm water that is entrained at Flemish Cap will have a large impact on the characteristics of the Slope Water downstream. The particular dynamics that control such an interaction are beyond the scope of this study.

\subsection{The vertical structure}

The surface analysis showed that SST anomalies in the Slope are, in part, the result of changes in the surface flow. Joyce et al. (2005) direct velocity measurements at $69^{\circ} \mathrm{W}$ showed that the vertical structure of the mean DWBC was rather barotropic with maximum velocities to the southwest of around $5-10 \mathrm{~cm} / \mathrm{s}$, comparable to our estimate of the mean speed of the SST anomalies in the Slope Water. Here, we focus on the variability. So next, we address the question: are the changes in the Slope Water the surface expression of changes occurring at depth?

We observed that low potential vorticity anomalies at $69^{\circ} \mathrm{W}$ were associated with increased SW flow of the Slope Water. To exploit of this co-variability we calculated 
the joint EOF of the velocity, temperature, planetary potential vorticity (PPV, defined here as $-(f / \rho) \partial \rho / \partial / z)$ and salinity from the hydrographic sections. The first mode (figure 2-11) explains $26 \%$ of the variability in the joint fields. A large fraction of the velocity variance at this location is known to be due to Topographic Rossby Waves (TRW) (Pickart and Watts (1990)). For a plane wave in the quasigeostrophic approximation, the temperature and horizontal velocity are $90^{\circ}$ out of phase. EOF1 has velocity and temperature in phase and thus the signal of the TRW is most likely represented in higher modes.

The surface signature of the velocity and temperature fields in EOF1 of the hydrographic data is consistent with the surface analysis, with colder temperatures associated with stronger southwestward flow. The vertical structure of the mode reveals that in this region the variability is surface intensified, but has a strong barotropic character (i.e., anomalies tend to be single signed at depth).

Temperature and salinity changes are well correlated, with lower salinities found in areas where temperature anomalies are negative, and higher salinities are found in areas of positive temperature anomalies. PPV reveals quite a different picture. The most striking feature of EOF1 is the large PPV anomaly centered at $800 \mathrm{~m}$ and extending 200km offshore occupying the upper Labrador Sea Water (uLSW) depth range. Low PPV anomalies are thus linked in this leading EOF to low temperature, low salinity and enhanced SW flow in the Slope Water.

The PPV mode in figure 2-11 has been normalized. If the normalization is removed and the resulting mode, now with full units $(m s)^{-1}$, is compared to the PPV calculated from the temperature and salinity components in EOF1, only minor differences are found. This indicates that the PPV mode extracted via the normalization is physically meaningful, and not an artifact of the manipulations performed to the data.

The agreement between the temporal structure of the vertical and surface modes is fair (bottom panel figure 2-8). The sign of the surface mode is well reproduced by the vertical mode, however the difference in the amplitude is rather large at times, especially during the 90s, when the surface PC1 exhibits some of its largest fluctuations, 

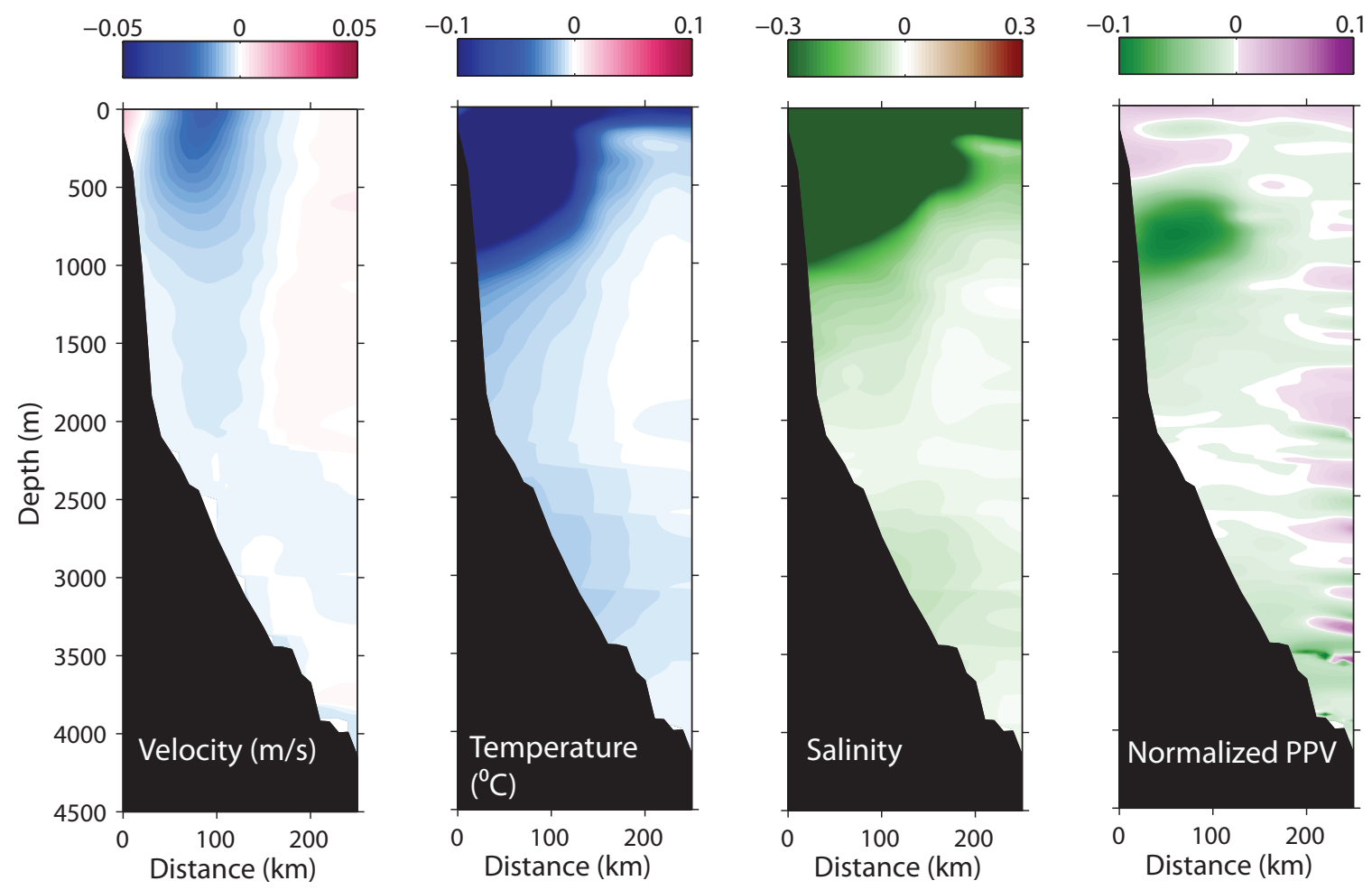

Figure 2-11: First EOF of the hydrographic sections at $69^{\circ} \mathrm{W}$. From left to right: anomalies of along-slope velocity $(\mathrm{m} / \mathrm{s})$, temperature $\left({ }^{\circ} \mathrm{C}\right)$, salinity $(\mathrm{psu})$ and normalized PPV $\left(\left(f \cdot N^{2}\right)_{i} /\left(\overline{f \cdot N^{2}}\right)_{i}\right.$, the overbar denotes time average and i denotes the element in the section).

while the vertical mode amplitude is minimum.

An earlier version of this analysis (Pena-Molino and Joyce (2008)) that included only 8 of the 13 sections analyzed here produced a mode with both spatial and temporal patterns somewhat different from those presented in this work that explained nearly $50 \%$ of the variance on the dataset. EOFs for small sample size, such is the case here, are highly dependent on the actual sample (eg. North et al. (1982) and von Storch and Hannoschock (1985)), it is therefore possible that the variability captured by this mode is dictated by the synoptic variability in the surveys, and not representative of the longer time scales. 


\subsection{Summary and discussion}

The dominant modes of variability in the Gulf Stream in the western North Atlantic correspond to changes in its position. The leading mode is a domain-wide latitude shift. During the time period of the observations, changes in position occur on interannual time scales, with alternating periods of about $2-3$ years duration in which the Gulf Stream occupies a more northerly or more southerly path. The second mode represents a change in the tilt of the Stream axis. During the positive phase of the second mode, the Gulf Stream path adopts a more southerly position near the separation point, and follows a more northerly track in the east, which causes the overall path to have a larger meridional tilt. In the negative phase, the situation reverses, the Gulf Stream moves to the north about the separation point and follows a nearly zonal path as it extends eastward. These changes occur at intraseasonal time scales, with a large fraction of the energy concentrated about 250 day period. A similar period of variability was reported by Lee and Cornillon (1995) for changes in the intensity of the meandering activity in the Gulf Stream. It is possible that path orientation and meandering activity in the Gulf Stream are linked by the changes in the stability properties of the Gulf Stream as it transitions from a more zonal to a more meridional path.

The latitude shifts of the Gulf Stream path captured by the first mode are correlated with SST anomalies in the Slope Water. The lag of this correlation is such that warm SST anomalies in the Slope Water precede northward shifts of the Stream by several months. The warming does not occur simultaneously over the entire region, but progressively from the region near Grand Banks to the separation point of the Gulf Stream at Cape Hatteras. This signal moves with speeds of several $\mathrm{cm} / \mathrm{s}$, consistent with typical Slope Water advection velocities.

The observed changes in SST could be due to a constant flow of water with changing temperature, a changing flow of water with constant temperature (the temperature of the source being different than the local temperature), or a combination 
of both. In the present analysis case we find that the Slope Water changes are a combination of both. As shown by the combined EOF of SST anomalies and surface geostrophic velocities, at times when the water over the Slope is colder (warmer), the SW flow intensifies (weakens). Except for the region under the direct influence of the Gulf Stream, east of the separation point, where velocity anomalies can at times reach $40 \mathrm{~cm} / \mathrm{sec}$, the largest velocity anomalies associated with the SST-velocity mode do not exceed $5 \mathrm{~cm} / \mathrm{sec}$. This upper bound for the velocity anomalies is smaller than the mean SW flow derived from the coherence between the SST anomalies and the Gulf Stream PC1 and the mean velocities observed along the Oleander track. This suggests that the mode of variability described here does not result in flow reversals but rather strengthening or weakening of the SW flow.

Some of the changes observed in the temperature field in the western North Atlantic appear to originate at higher latitudes. Using complex EOF we find that SST anomalies propagate from the central Labrador Sea along the continental slope to subtropical latitude. The amplitude of this propagating feature increases as the signal makes the turn around the Grand Banks. We believe this amplification may be related to the interaction between the upper flow in the DWBC and the Gulf Stream/North Atlantic Current.

The leading mode of joint variability of the velocity, salinity and temperature inferred from the hydrographic data at $69^{\circ} \mathrm{W}$ shows that the stronger fluctuations occur in the upper $1000 \mathrm{~m}$ of the water column. While the amplitude of the fluctuations decreases with increasing depth, the dominant mode is single signed in depth. The phase relationships between the velocity, salinity and temperature anomalies captured by this mode agree with those found by Flagg et al. (2006). These authors showed that a large fraction of the interannual variability in the temperature and salinity of the upper $500 \mathrm{~m}$ of the Slope Water could be explained by advection with cold and fresh periods being the result of stronger SW flow.

The Gulf Stream path PC1 and the first rotated principal component of the combined SST-velocity are anti-correlated, meaning that when the Gulf Stream takes a 
more northerly position, the SW flow in the Slope is weaker and the SST warmer. The maximum value of the correlation, - 0.6 , is found when temperature and velocity anomalies lead the GS path changes by 6 months. This lag is smaller than that reported by Zhang and Vallis (2006). They found that northward shifts of the Gulf Stream followed positive temperature anomalies by about 1 year. This disagreement might be partially explained by the spatial differences in the lag which the EOF is unable to capture.

The in-phase relationship between variability in the velocity field at the surface and at depth implies that stronger SW surface velocities are the surface signature of larger DWBC transports. Therefore decreasing temperatures and increasing transport in the DWBC lead, according to the modes described here, to southward shifts of the Gulf Stream axis. This agrees with the relationship found between SST in the slope and fluctuations in the Gulf Stream mean axis and supports the results of Thompson and Schmitz (1989).

The surface velocities in our analysis do not exhibit a trend. Häkkinen and Rhines (2004) found that the slowing of the circulation in the subpolar North Atlantic they observed did not extend to middle latitudes. The fluctuations from the trend in their SSH PC1 are in good agreement with the temporal patterns shown here, and suggest that this mode of variability may have a basin wide signature.

According to our results, changes in the properties and circulation of the Slope Water are observed preceding latitude shifts in the Gulf Stream axis. These changes that affect the entire water column and move SW along the Slope can be observed at the surface and may therefore provide predictability for Gulf Stream path changes. 


\section{Chapter 3}

\section{Recent changes in the Labrador Sea Water within the Deep Western Boundary Current Southeast of Cape Cod}

\subsection{Introduction}

In the traditional view of the North Atlantic Deep Water (NADW) circulation, the Deep Western Boundary Current (DWBC) was considered to be the main pathway from the high-latitude formation regions into the subtropical domain. The existence of the DWBC was first predicted by Stommel (1958) who invoked a deep boundary current to close the abyssal circulation. But it was not until 1960 that the DWBC current was observed for the first time (Swallow and Worthington (1961)). The intermediate depths of the North Atlantic's DWBC are occupied by Labrador Sea Water (LSW). This water mass is believed to originate in the Labrador and Irminger Seas as well as in the Labrador current (Pickart et al. (1997)). Modeling studies suggest that changes in the Meridional Overturning Circulation (MOC) are related to the rate of production of LSW (e.g. Mauritzen and Häkkinen (1999), Bailey et al. 
(2005), Böning et al. (2006)).

At mid-latitudes in the North Atlantic, the mean DWBC appears as a wellorganized current with little vertical shear and typical velocities of 5 to $10 \mathrm{~cm} / \mathrm{s}$ (Joyce et al. (2005) and Toole et al. (2010)). Looking closer, observations at the exit of the subpolar gyre (e.g. Schott et al. (2004), Schott et al. (2006) and Pickart and Smethie (1998)), reveal that the DWBC has two separated velocity cores, a shallow one between 500 and 2000m, and a deeper one center around 3000m. About 40N, the steepness of the continental slope and the relative position of the Gulf Stream, cause the two cores to appear aligned and the overall flow in the DWBC to look more barotropic.

The upper part of the water column above the DWBC, extending from the surface to the upper limit of the LSW is typically referred to as Slope Water and represents a mixture of very fresh and cold shelf water originating in the Subpolar North Atlantic and transported into the subtropics by the Labrador Current, and waters from the Northern Reciculation Gyre (NRG) of the Gulf Stream. The mean flow in this upper part of the water column has very similar speeds to those found in the DWBC underneath it (Flagg et al. (2006)). Underneath the Slope Water, the uppermost layer of the DWBC, extending from 500m to $1000 \mathrm{~m}$ approximately, is occupied by upper Labrador Sea Water (uLSW). uLSW may be formed by convection near the boundary in the Labrador Sea (Pickart et al. (1997)) or in the middle of the basin during less severe winters (Stramma et al. (2004), Yashayaev (2007)). Because convection down to the level of uLSW can occur under moderate winter conditions, this water mass is thought to be formed every year (Pickart et al. (1997)) unlike its deeper counterpart, the deep Labrador Sea Water (dLSW). The dLSW occupies the intermediate depths of the DWBC, typically from $1000 \mathrm{~m}$ down to $2500 \mathrm{~m}$. dLSW is a thicker layer of much more homogeneous fluid, since it is formed in the middle of the Labrador Basin only during very harsh winters when convection reaches down to $2000 \mathrm{~m}$ or more. The densest component of the NADW within DWBC below the dLSW, typically referred to as Overflow Water (OW), is formed in the Nordic seas (e.g. Worthington (1976) 
and Pickart (1992)).

Because the water masses in the DWBC are formed by convection at high latitudes, once they sink and are no longer in contact with the atmosphere, their potential vorticity tends to be conserved. It is this insulation from surface forcing and the low PV values that characterize water of convective origin, that allow LSW to be traced by its PV minimum. Based on maps of the North Atlantic PV, Talley and McCartney (1982) identified three main pathways for the newly-formed LSW: (1) northeastward into the Irminger Sea, (2) southeastward underneath the North Atlantic Current and (3) southward via the DWBC . Similar results were found by Rhein et al. (2002) based on CFC distribution and by Bower et al. (2009) from float observations.

The continental slope bathymetry along the east coast of the US between Grand Banks and Cape Hatteras acts to constrain the path of the DWBC to lie within one hundred kilometers of its mean position over the continental slope. For that reason is relatively straightforward to monitor the DWBC transport here as compared to $26.5^{\circ} \mathrm{N}$, where the DWBC sits above a relatively flat bottom and exhibits large offshore shifts (Bryden et al. (2005)).

The mean transport of the DWBC along its path in the North Atlantic exhibits some variation. East of the Grand Banks, Schott et al. (2006) estimated that the mean transport of the DWBC below the $\sigma_{\theta}=27.68 \mathrm{~kg} / \mathrm{m}^{-3}$ (upper boundary of the uLSW) was $-17.5 \pm 6.8 \mathrm{~Sv}$. As the NADW enters the subtropical domain, at $55^{\circ} \mathrm{W}$, Pickart and Smethie (1998) obtained a slightly larger mean transport of $-18.9 \pm 6.3 \mathrm{~Sv}$ for the same water masses. Further downstream at $70^{\circ} \mathrm{W}$, Joyce et al. (2005) reports an Eulerian mean of $-16.5 \pm 2.5 \mathrm{~Sv}$ (the error being related to the presence of Warm Core Rings in their section), similar (within the errorbars) to the more recent estimates by Toole et al. (2010) from 4 years of moored velocity measurements at the same site $(-18 \mathrm{~Sv}$ 4-layer Eulerian mean, -25.1Sv when averaged in stream coordinates). On approach to Cape Hatteras, most of the DWBC waters in the upper part of the water column, including some within the LSW depth range, are entrained by the Gulf Stream and recirculate back into the mid-Atlantic Bight. Only the deepest components manage 
to cross under the Gulf Stream (Bower and Hunt (2000) and Pickart and Smethie (1993)). South of the cross-over the DWBC recovers some of the lost transport via recirculation, and by $26.5^{\circ} \mathrm{N}$ the mean transport is increased in magnitude to -35 to $-40 \mathrm{~Sv}$, half of which are recirculating waters (Lee et al. (1996) and Bryden et al. $(2005))$.

Our knowledge of the temporal variability of the DWBC transport is much less complete due to the lack of long term measurements. The available records show that the DWBC velocity field is highly variable on intraseasonal scales, and it is only the density field that shows clear interannual variations (Schott et al. (2006) and Vaughan and Molinari (1997)).

Recent observational studies suggest that the fraction of the LSW exported via the DWBC may not be as large as believed, thus its contribution to the MOC not as important. Fischer and Schott (2002) found that none of the profiling floats deployed in the Labrador Sea in the Spring and Summer of 1997 were able to navigate the rough topographic features around Flemish Cap and enter the subtropical domain. Instead, all of their floats recirculated back into the Labrador Sea, or flowed eastward toward the Charlie Gibbs Fracture Zone. In a more recent study, Bower et al. (2009) deployed a number of RAFOS floats in the period 2003-2006 at the LSW depth within the Labrador Current and obtained very similar results. Only a small percentage $(8 \%)$ of the floats deployed in LSW depth range north of the Grand Banks followed the classic path along the continental slope to subtropical latitudes. Most of the floats recirculated back into the Subpolar North Atlantic. Among the few that did eventually travel south, most did it via an interior pathway. It is important to mention that both these studies took place during weak to no-convection conditions in the Labrador Sea (Yashayaev (2007)).

In this study we use data from a series of Moored Profiler mooring deployments in the DWBC downstream from the Grand Banks in combination with hydrographic survey and altimetry data to characterize the variability at this site and investigate changes in the NADW, with particular focus on the LSW. We will relate the observed 
changes to changes in dense water formation in the subpolar North Atlantic. The paper is organized as follows: first we introduce the data set. A description of the means and anomaly fields measured by Moored Profilers, and the patterns of co-variability between the density and velocity fields follows. Next we explore the variability in the transport as seen at the single mooring site and discuss what the possible sources of this variability are. And then before the discussion, we describe changes in the water masses and relate them to their different spreading rates away from the formation region.

\subsection{The dataset: W3 deployment.}

The bulk of the data used in this study were obtained from a series of mooring deployments at a site located southeast of Cape Cod on the continental slope near the $3000 \mathrm{~m}$ isobath at approximately $39^{\circ} \mathrm{N} 69^{\circ} \mathrm{W}$ (hereafter W3, see figure $3-1$ ). The moorings were equipped with a McLane Moored Profiler (MMP) that measures conductivity/temperature/pressure and horizontal velocity, and some additional fixeddepth sensors: Vector Averaging Current Meters (VACM) and MicroCAT's, at the top and bottom of the mooring. The record examined here consists of one deployment in 2001-2002 that returned profile data from November 2001 to August 2002, and 5 sequential one-year deployments (from April 2003 to May 2008). During 20042008, W3 was deployed together with 4 other moorings as part of the Line W program (http://www.whoi.edu/science/PO/linew/index.htm) in an effort to monitor the transport of the DWBC in the western North Atlantic. Results from the full array deployment are presented by Toole et al. (2010) and Chapter 4 of this thesis.

After processing (for details on the processing see Toole et al. (2010)), the MMP data set consists of 2 dbar vertical resolution profiles of temperature, salinity and horizontal velocity data. The MMP's were programmed to sample in bursts, with a burst typically consisting of 4 profiles spaced 9 hours apart, and bursts separated 5 days. In order to filter tidal and inertial motions, all profiles within a burst were averaged 


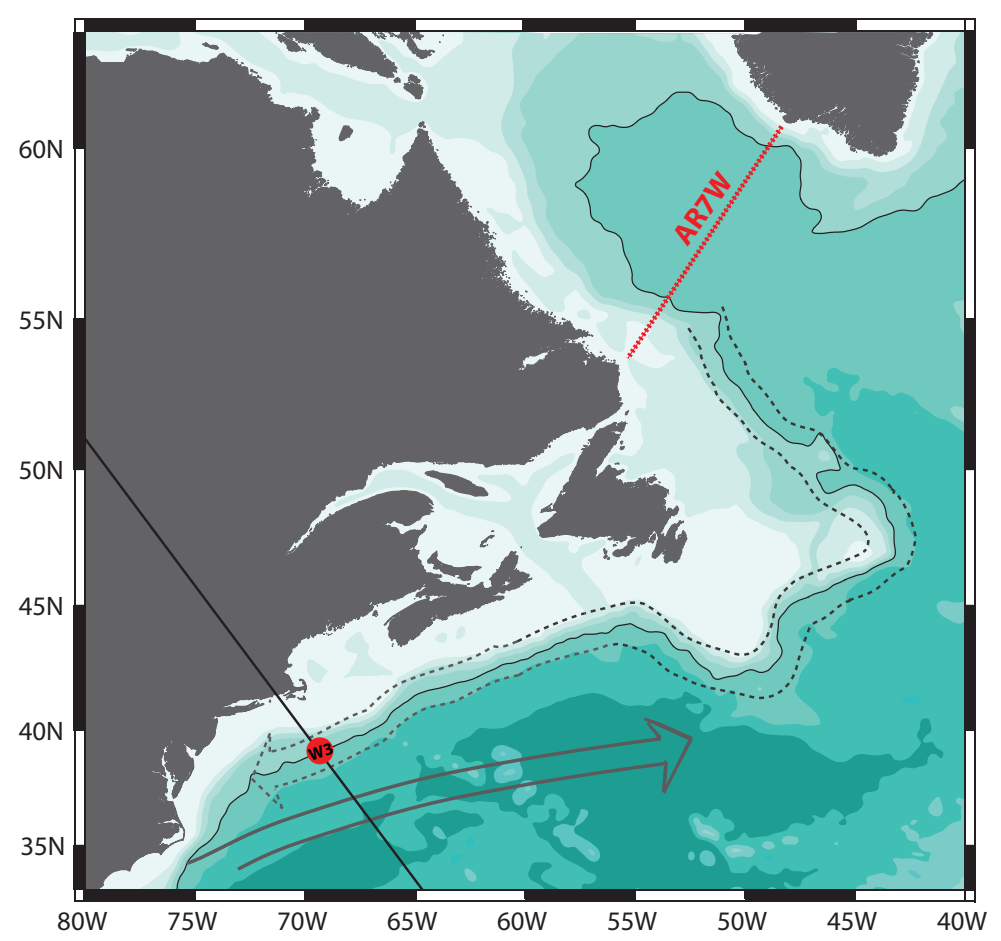

Figure 3-1: Schematic showing the Gulf Stream (solid line arrow) carrying warm water to the north, side to side with the DWBC (dashed line arrow), carrying cold waters to the south along the continental slope, in the area of study. The thin black line is the altimeter track 126 . The location of W3 is shown by the red circle. WOCE line AR7W in the Labrador Sea is shown in red.

(Silverthorne and Toole (2009)), thus the final temporal resolution is given by the frequency of the bursts rather than that of the individual profiles. These and other details for each deployment are shown in Table 3.1. One of the most valuable attributes of the MMP data is its vertical resolution, allowing us to accurately compute planetary potential vorticity (PPV, defined here as $(f / \rho) \partial \rho / \partial z)$, a key variable in the study of water masses of convective origin. An important limitation of the dataset is missing data. In the presence of strong currents the MMPs sporadically returned incomplete profiles or failed to profile as scheduled, and often exhausted their battery supply a month or so before the mooring was serviced.

Some post-processing was performed on the data before the present analysis. Missing data were treated as follows. In the vertical, incomplete profiles that were missing more than $30 \%$ of the profile span (normally at the top and/or bottom of the moor- 
Table 3.1: W3 mooring deployments.

\begin{tabular}{lll}
\hline \hline Time & MMP depth & Burst scheme \\
\hline $11 / 2001-06 / 2002$ & $94-2960 \mathrm{~m}$ & 4-profiles, 4-day \\
$05 / 2003-04 / 2004$ & $74-2950 \mathrm{~m}$ & 4-profiles, 5-day \\
$04 / 2004-05 / 2005$ & $60-3150 \mathrm{~m}$ & 4-profiles, 5-day \\
$05 / 2005-04 / 2006$ & $104-3220 \mathrm{~m}$ & 4-profiles, 7.5-day \\
$04 / 2006-04 / 2007$ & $60-3192 \mathrm{~m}$ & 4-profiles, 5-day \\
$04 / 2007-05 / 2008$ & $60-3190 \mathrm{~m}$ & 6-profiles, 5-day \\
\hline
\end{tabular}

ing), were excluded from this analysis. For the remaining partial profiles, VACM and MicroCAT data were used to fill in the gaps using linear interpolation. Small temporal gaps (smaller than 14 days) were also filled by linear interpolation; larger gaps were masked. The resulting data were then gridded onto a 7-day common time axis, and only the depth range sampled by all 6 mooring deployments was retained.

One last step in the post-processing involved identifying and excluding Warm Core Ring (WCR) events. W3 is located north of the mean Gulf Stream but close enough that large Gulf Stream excursions (rings and meanders) can sometimes block and reverse the otherwise equatorward DWBC flow at W3. These excursions represent a significant fraction of the variability in the region and have a large impact on the record-mean temperature and salinity. Defining anomalies relative to the fullrecord mean, including the rings, produces temperature and salinity anomaly fields that consist of positive anomalies (warm and saline) when the rings are present, and negative anomalies (cold and fresh) during ring-free periods. It was therefore necessary to exclude the rings to isolate the internal variability of the water masses that constitute the NADW beyond the effects of the rings. The large horizontal velocities associated with the rings often prevented or limited the ability of the MMPs to profile and Ring periods were largerly already eliminated from the present data set. In those cases when the MMP was able to profile during all or part of a ring event, the data from those periods were manually eliminated. To determine when a set of profiles was contaminated by a ring, we took advantage of the large temperature 
anomalies associated with warm core rings, and eliminated profiles that contained temperature anomalies from the full record mean profile with amplitudes three times (or more) larger than the standard deviation of the time series.

In addition to the mooring data, six hydrographic sections along Line $\mathrm{W}$ and absolute Sea Surface Height (SSH) for T/P-Jason track 126, coinciding with Line W, made available by AVISO (http://www.aviso.oceanobs.com/duacs/) were utilized. The absolute dynamic topography data from AVISO was used to calculate surface geostrophic velocities in the direction normal to the altimeter track. Information about the hydrographic surveys is provided in Table 3.2.

Table 3.2: Hydrographic surveys used for the composite sections.

\begin{tabular}{|c|c|c|}
\hline & Cruise \# & Date \\
\hline \multirow{3}{*}{ Composite 1} & Kn173_2 & Nov 08-Nov 13, 2003 \\
\hline & Oc401 & Apr 28May 06, 2004 \\
\hline & Kz124 & Sep 04-Sep 12, 2004 \\
\hline \multirow{3}{*}{ Composite 2} & Oc432 & Oct 19-Oct 26, 2006 \\
\hline & En440 & Oct 01-Oct 09, 2007 \\
\hline & Oc446 & May 10May 20, 2008 \\
\hline
\end{tabular}

\subsection{Evolution of the hydrographic properties and circulation at W3}

\subsubsection{The mean and the anomaly fields.}

Temperature, salinity, PPV and downstream velocity ${ }^{1}$ anomalies in depth space were obtained by removing the record mean profile after rings were excluded. The anomaly fields were subsequently normalized by the standard deviation at each depth level to account for the larger variances observed in the upper ocean relative to the abyss.

\footnotetext{
${ }^{1}$ Rotated into the along-isobath direction, ca. $31^{\circ}$ counterclockwise from the east. Positive velocities are to the northeast, and negative to the southwest.
} 
Results presented here do not change qualitatively if the normalization is not performed. Temperature, salinity, PPV and downstream velocity anomalies, as well as mean profiles, are shown in figure 3-2.

The mean density field at W3 is typical for the Slope Water region in the western North Atlantic with a relatively shallow pycnocline compared to the stratification just a few hundred km further offshore south of the Gulf Stream, and a PPV and salinity minima at mid depth indicative of the presence of LSW. At 220m depth, mean temperature values are around $10^{\circ} \mathrm{C}$ (waters to the north of the Gulf Stream are typically around $12-13^{\circ} \mathrm{C}$ at $150 \mathrm{~m}$ ) bounded below by a steep thermocline followed by a gradual temperature decrease all the way to the bottom, where potential temperatures of about $2^{\circ} \mathrm{C}$ are found. Mean salinity values range from 35.4 at $220 \mathrm{~m}$ to 35 at the base of the thermocline. Below the thermocline, salinity changes are much smaller, with a local minimum at the LSW layer, co-located with a PPV minimum. The mean velocity is to the southwest at all depths, with maximum velocities of $5 \mathrm{~cm} / \mathrm{s}$ at the top and a nearly linear vertical shear that brings the record mean velocity down to $3.5 \mathrm{~cm} / \mathrm{s}$ near the bottom.

The variability in the temperature and salinity fields is characterized by a marked change in the sign of the anomalies from the first to the second half of the record, with the early years being on average warmer and more saline than the later ones. These warm (cold) and saline (fresh) anomalies don't occupy the entire water column, but rather have a vertical structure consisting of alternating layers of warm (and saline) and cold (and fresh) water roughly contained within the depth range of uLSW, dLSW and OW (see figure 3-2). Anomalies in the uLSW and OW are in phase, with warm and saline anomalies from 2001 to 2003 and cold and fresh anomalies from 2006 until the end of the record. Anomalies in the dLSW depth range have the opposite sign. In the transition period between 2004 to 2006 the anomalies appear nearly depth independent.

The most interesting of the signals are found in the PPV field. There is a clear change in the vertical structure of the PPV around 2004. The first half of the record 

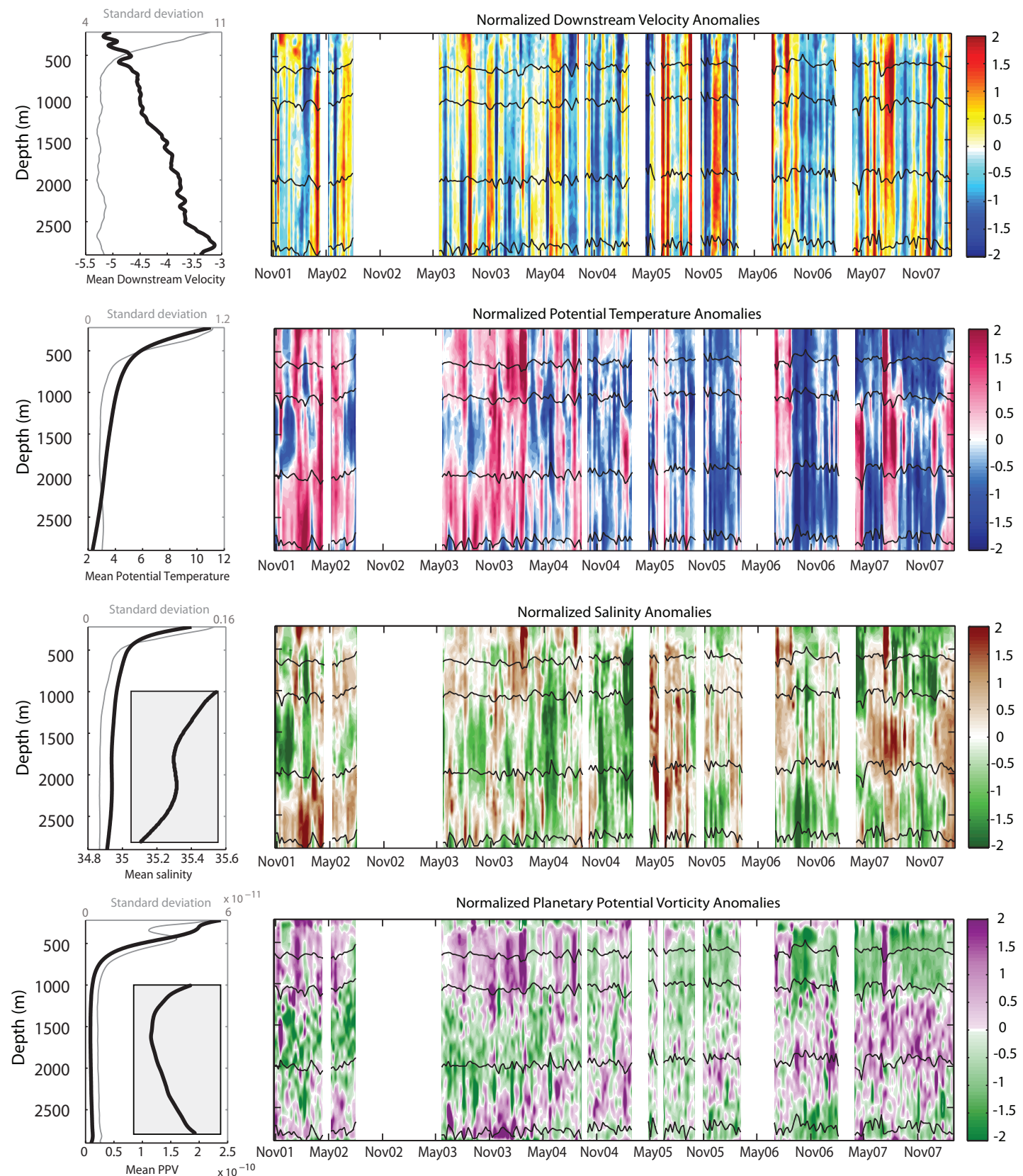

Figure 3-2: The color maps on the right show normalized time series of anomalies, from top to bottom, downstream velocity, potential temperature, salinity and planetary potential vorticity (PPV) at W3. In black, the boundaries, neutral density surfaces shown in table 3.3, for uLSW, dLSW and OW. Left panels show the mean, thick black line, and standard deviation (scaled to match the mean scale), thin gray line, profiles for the corresponding variables. A blow-up of the bottom 2000m of mean PPV and salinity profiles are shown in the shaded box, showing the minima PPV and salinity minimum in the dLSW layer. 
is characterized by a thick layer of relatively low PPV at the level of the dLSW and a more stratified uLSW layer. In the second half, the situation reverses. The PPV minimum gradually migrates upwards to occupy the whole range of uLSW and the part of the water column right above it. The stratification in the dLSW increases during this time. The timing of this transition from a denser type of LSW to a lighter version is consistent with the evolution of the LSW observed in the Labrador Sea about a decade earlier. Stramma et al. (2004) found from a series of hydrographic observations taken between 1996 and 2001, that after the exceptionally deep convection in the central Labrador Sea ceased in the mid 1990's, the dLSW layer became thinner, while the uLSW layer, almost absent before 1996, increased its thickness up to 2001. The same transition from the denser to the lighter LSW observed here at 69W around 2004 was observed in the central Labrador Sea in 1998 (Yashayaev (2007)). This implies an averaged water parcel transit time from the Labrador Sea to W3 of 6 years, equivalent to a speed of $2.5 \mathrm{~cm} / \mathrm{s}$ following the $3000 \mathrm{~m}$ isobath.

\subsubsection{Vertical modes of co-variability.}

To examine the joint variability in the downstream velocity, potential temperature, salinity and planetary potential vorticity fields we performed a multivariate EOF decomposition. We proceeded in several different ways, as explained next in some detail to justify our final choice of the physically-meaningful leading mode. Given the apparent different nature of the variability in the velocity field and the remaining variables, we first excluded the velocity data from the analysis, and calculated the joint EOF of temperature, salinity and PPV alone. We will refer to this case as $E O F_{3 V A R}$. The leading mode of the $E O F_{3 V A R}{ }^{1}$ explains $12 \%$ of the total variance and it is well separated in amplitude from succesive modes. The mode is characterized by cooler

\footnotetext{
${ }^{1}$ This mode is not shown here, but the spatial patterns in temperature, salinity and PPV shown in figure 3-3 for the analysis denoted $E O F_{4 V A R-r o t}$ that is explained later, are, for all practical purposes, identical to those described now. The same is true for the corresponding principal component, PC1.
} 
(warmer) and fresher (more saline) water at all depths, with slightly smaller amplitude at mid detph, in particular at $1500 \mathrm{~m}$ for temperature and $2000 \mathrm{~m}$ for salinity. In PPV the mode consists of alternating layers of low-high-low anomalies with interfaces (zero-crossings) at 1500 and $2500 \mathrm{~m}$. The principal component of the leading mode, PC1, is dominated by a sign change at the begining of 2004 going from a mostly negative (warmer/saline) early part of the record to a mostly positive (colder/fresh) in the second half. The spatial and temporal characteristics of this leading mode remain unchanged when the EOF analysis is performed on each of the three variables individually, which is a sign of the robustness of the mode.

In order to extract the fraction of the downstream velocity variability that co-varies with the other three variables, PC1 from the $E O F_{3 V A R}$ analysis was regressed onto the velocity field at each depth. The regression coefficients provide an equivalent spatial pattern of the EOF, denoted $E O F_{V-r e g}$ (not shown here). $E O F_{V-r e g}$ is characterized by negative velocities down to $2500 \mathrm{~m}$, with little vertical shear, and a sign reversal in the bottom 500m (in-phase with positive PPV anomalies at mid-depth). However, in the regression the information about the percentage of the variance that is captured by the mode is lost, thus only the mode's structure, not its amplitude, is physically meaningful.

To recover the amplitude in the velocity mode we included all four variables in a subsequent decomposition: $E O F_{4 V A R}$. In this case, the first, second and third modes explained about $9 \%, 7 \%$ and $5 \%$ of the total variance, respectively. The temporal and spatial patterns for T, S and PPV from $E O F_{4 V A R}$ (figure 3-3) are very similar to the $E O F_{3 V A R}$ case. The velocity mode has similar vertical structure to $E O F_{V-r e g}$ but lacks the sign reversal in the bottom 500m. However, the proximity between the eigenvalues of the first three leading modes indicates there is potential for mode mixing. The second mode (not shown here) has a very similar structure for $\mathrm{T}, \mathrm{S}$ and PPV but opposite sign in velocity, thus the fraction of the velocity field that covaries with the other variables could be much smaller than what is captured by $E O F_{4 V A R}$ if the first and second modes are combined. To separate the mixed modes, we performed 
a VARIMAX rotation (von Storch and Zwiers (1999)) of the 3 leading modes of the $E O F_{4 V A R}$ case. We will denote the rotated mode $E O F_{4 V A R-r o t}$. The resultant T, S and PPV leading modes are identical to $E O F_{3 V A R}$ (figure 3-3). The velocity pattern has the same exact vertical structure as $E O F_{V-r e g}$ (including the sign reversal), but an amplitude 5 times smaller. PC1 of both $E O F_{3 V A R}$ and $E O F_{4 V A R-r o t}$ are also the same.

While the PPV mode clearly captures the vertical structure of positive and negative anomalies discussed earlier, the temperature and salinity modes failed to do so. The amplitude of the mode at intermediate depths decreases, but the sign remains constant. These depth-independent temperature and salinity change can be driven by changes in the rate of entrainment of warm and saline Gulf Stream waters as the LSW progresses southward. But it could also be an artifact of the EOF's tendency to select the normal modes of the system $\left(n^{\text {th }}\right.$-mode having $(n-1)$ zero-crossings $)$ (North (1984)).

The amplitude of the rotated velocity mode, once the normalization is undone, is one order of magnitude smaller than the amplitude of the full velocity fluctuations. Considering the mean downstream velocity profile $(3.5-5 \mathrm{~cm} / \mathrm{s})$ the fluctuations that are captured by this mode represent approximately $25 \%$ of the mean flow's amplitude. This implies that if we add or subtract the rotated velocity mode times one standard deviation of the principal component, the mean velocity profile will accelerate or slow down by $1 \mathrm{~cm} / \mathrm{s}$ (see figure $3-4$ ). When the mode is subtracted from the mean, the strength of the flow (southwestward) in the upper 2000m decreases, while it increases in the bottom $500 \mathrm{~m}$. This results in an overall reduction of the shear. The opposite is true when the mode is added to the mean. When the mode reaches its maximum amplitude $(\mathrm{PC} 1 \approx 2)$, the velocity anomaly is still only $50 \%$ of the amplitude of the mean flow, which is not large enough to cause flow reversals.

Based on $E O F_{4 V A R-r o t}$, only $10 \%$ of the variability in the flow is related to changes in the density field captured by the PPV mode 1 . The dominant mode of variability of the velocity alone, $E O F_{V}$, is also shown in figure $3-4$ ( $45 \%$ of the 

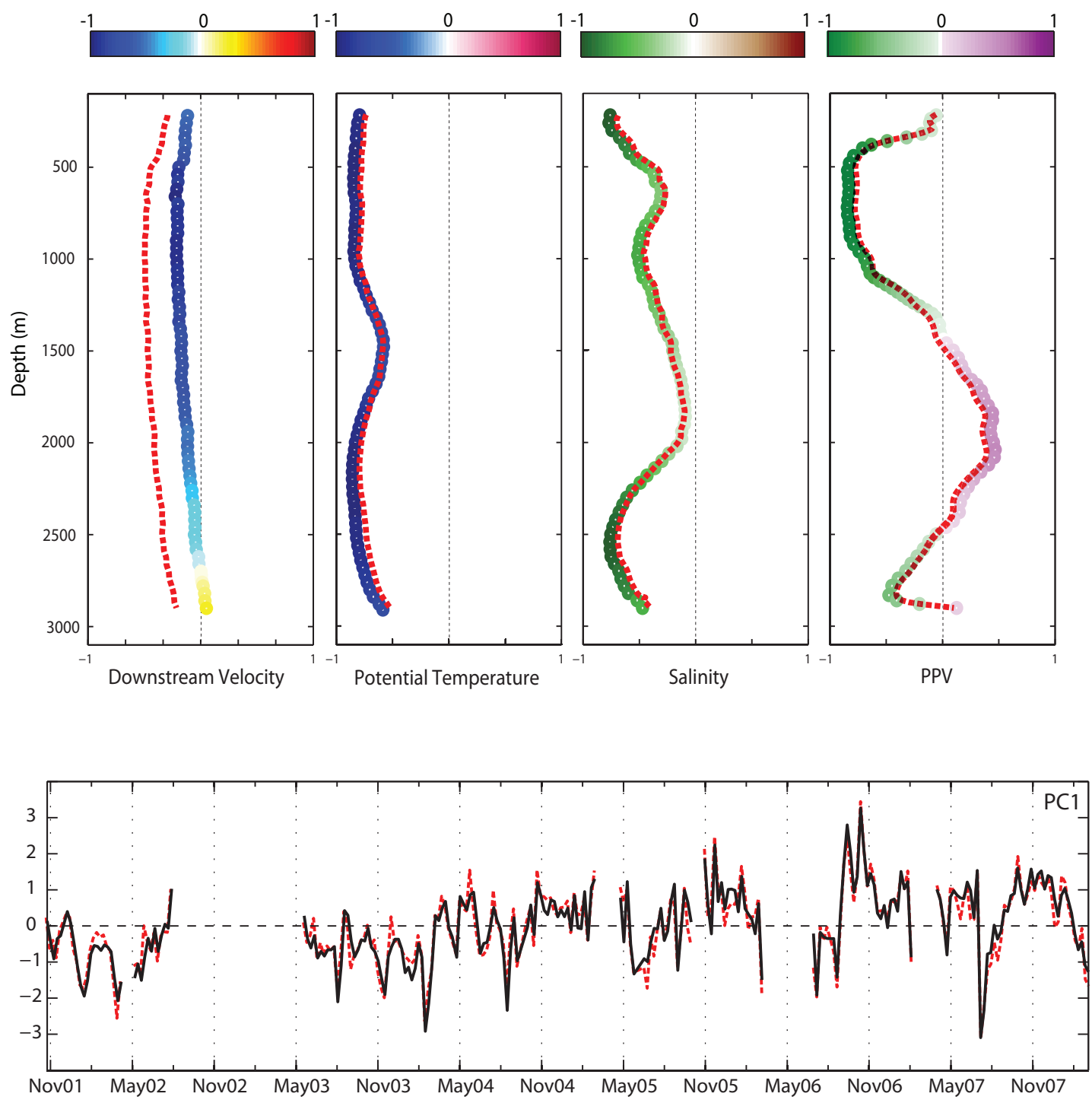

Figure 3-3: Top panels show in color the leading EOF of the downstream velocity, potential temperature, salinity and PPV and the VARIMAX-rotated case, $E O F_{4 V A R-r o t}$. The color as well as the horizontal axis show the amplitude of the normalized anomalies captured by the mode, ranging from -1 to 1 . The red dashed line is the leading EOF when no rotation is performed, EOF $4 V A R$. The amplitude and sign of the patterns captured by these EOFs change over time according to their corresponding principal components, shown in the bottom panel. The solid black line corresponds to the PC1 for the VARIMAX-rotated case, EOF $4 V A R-r o t$, and the red dashed line for the one with no rotation, $E O F_{4 V A R}$. 

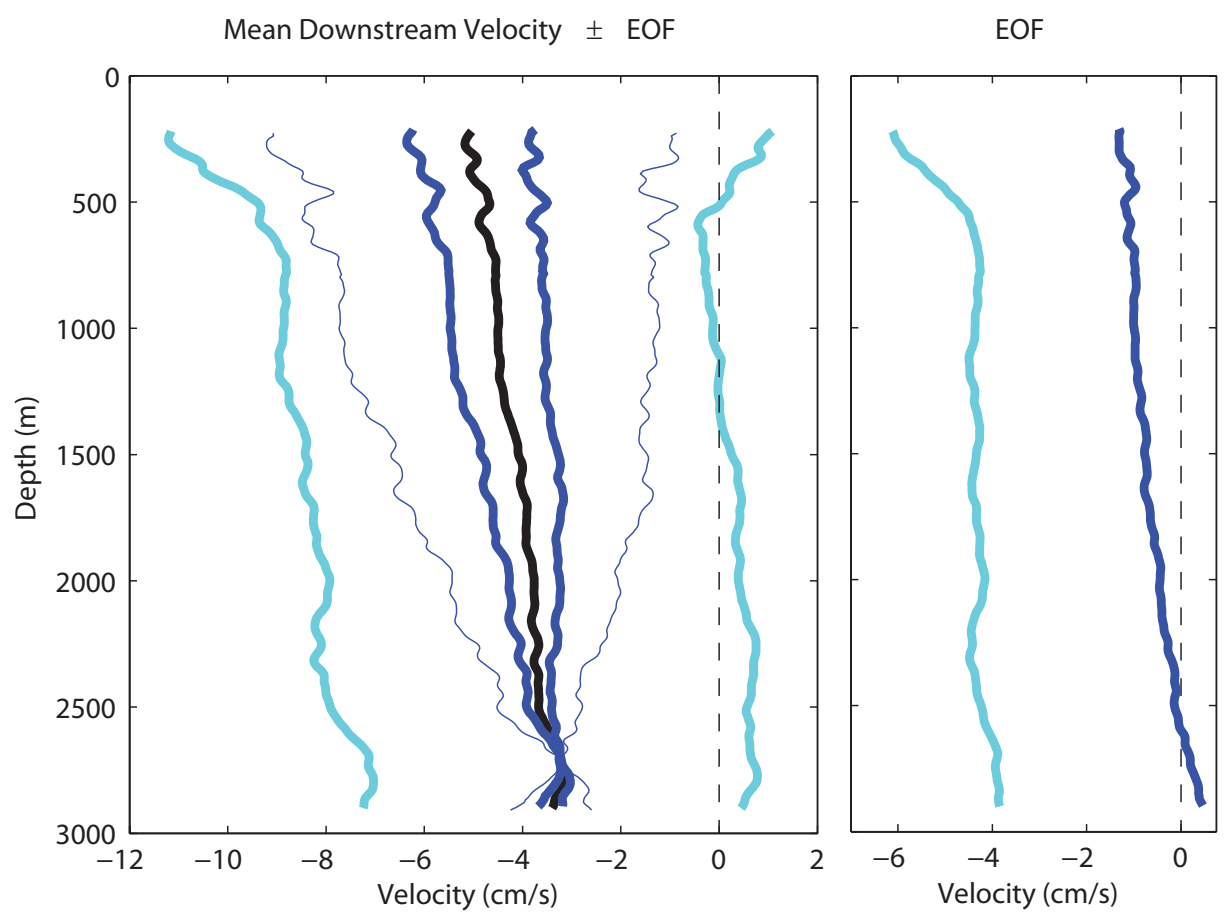

Figure 3-4: The right panel represents the re-scaled (real velocity units) downstream velocity leading $\mathrm{EOF}$ for the rotated joint case, $E O F_{4 V A R-r o t}$, in blue, and for the velocity alone, $E O F_{V}$, in cyan. In the left panel the mean downstream velocity is shown in black, the mean $\pm E O F_{V}$ in cyan, and the mean $\pm E O F_{4 V A R-r o t}$ and $\pm \operatorname{Max}(P C 1) \times E O F_{4 V A R-r o t}$ in thick and thin blue lines respectively. At $2800 \mathrm{~m}$, $E O F_{4 V A R-r o t}=0$ and the four combinations of mean plus mode converge to the mean value.

variance). When the amplitude of this mode is multiplied by the standard deviation of the corresponding PC1 (not shown here), $P C 1_{V}$, is comparable to the amplitude of the mean. Subtracting this mode from the mean can produce a flow reversal in the deeper part of the water column where the mean flow is weaker. At times when $P C 1_{V}$ is large (twice the standard deviation) this mode can reverse the flow in the entire water column. $P C 1_{V}$ fluctuates at higher frequencies than does the joint mode. Some of these fluctuations are related to changes in the direction of the flow, not on its strength. The departures of the direction of the instantaneous flow from the downstream direction are rather large. Only when the velocity is averaged over periods longer than 6 months does the direction of the flow start to converge to that of the mean. Year-to-year differences in the annually averaged flow are comparable in 
amplitude to the variability captured by the joint EOF. So we can think of the joint EOF as a filter that is selecting only the fluctuations that represent the low-frequency changes in the strength of the flow and not in its direction.

In the deeper part of the water column, some of the high frequency variability associated with directional changes is due to Topographic Rossby Waves (TRW). Their presence in this area is well documented in the literature (e.g. Thompson and Luyten (1976), Pickart and Watts (1990), Pickart (1994) and Frantantoni and Pickart (2003)). TRW's are bottom intensified with periods at Line W ranging from 25-40 days. In the upper $1000 \mathrm{~m}$, most of the observed directional changes are related to Gulf Stream rings and meanders. These can be seen in the surface geostrophic velocities estimated from the altimeter record, figure 3-5a. The altimeter velocities are normal to the altimeter track (downstream direction), and contain no information about the along-track (cross-stream) component, and therefore of the actual direction of the flow. However, the amplitude of the downstream velocity fluctuations associated with Gulf Stream rings and meanders is much larger than the typical velocity fluctuations at W3, and so rings and meanders can be seen as large spikes in the altimeter record.

The time series of surface geostrophic velocities compares well with the velocities measured by W3 at $220 \mathrm{~m}$. There is an offset of a few $\mathrm{cm} / \mathrm{s}$ with the altimeter being more positive (weaker SW flow) than the direct velocity measurements at W3. 200km to the southwest along the $3000 \mathrm{~m}$ isobath where $\mathrm{W} 3$, downstream velocities obtained from the Oleander shipboard ADCP data (a ship of opportunity ship running between New Jersey and Bermuda, see Rossby and Gottlieb (1998) for details) show that the mean vertical shear is not large enough to explain this offset. We believe this offset is due to the large degree of smoothing of the mean dynamic topography. When the respective mean velocities are removed, the agreement between the anomalies in the altimeter and W3 time series is much better. However, the full altimeter record alone cannot see the low frequency variability captured by W3 -it is dominated by rings and meanders. The principal mode of variability of the combined altimeter/SST record, in the other hand, does exhibit low-frequency signals. As shown in Chapter 2 by the 

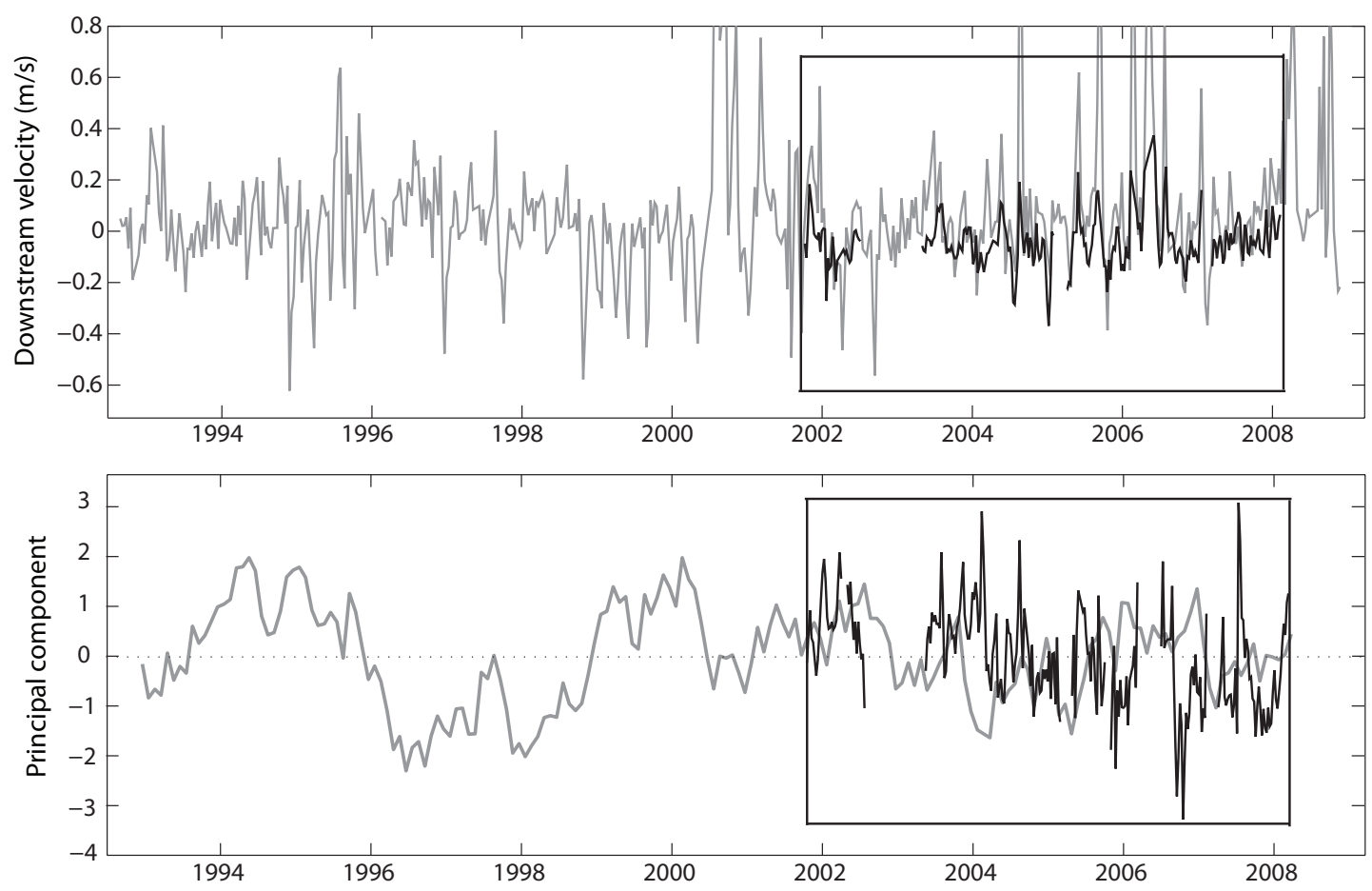

Figure 3-5: Top panel shows in gray surface geostrophic velocities from the altimeter at the location of mooring W3. In black is the time series of downstream velocity at 220m from the W3 MMP record. Both in m/s. Bottom panel shows in gray PC1 of joint EOF of the temperature and surface geostrophic velocity in the Slope Water region from Chapter 2 (bottom panel figure 2-8). In black, PC1 from the VARIMAXrotated temperature, salinity, PPV and downstream velocity EOF from W3 (same as in figure 3-3).

joint EOF of surface geostrophic velocities and sea surface temperature (SST) in the Slope Water, cold (warm) anomalies are accompanied by intensification (weakening) of the southwestward flow, similar to what is found here in the subsurface. Comparing the principal component of the surface analysis in Chapter 2 to the multivariate PC1 from W3, the overall trend is similar, with both showing negative values for the early 2000's and positive in subsequent years (see figure 3-5B). However, there are differences between the two approaches, likely due to different analysis time periods and different input data. 


\subsection{The transport response to changes in the LSW}

One of the interesting results from the EOF analysis is the in-phase/out-of-phase relationship between the velocity and the PPV for the uLSW and dLSW. While the PPV anomalies in the upper and deep LSW have opposite sign, the corresponding velocity anomalies have the same. Considering the temporal pattern of the mode, PC1, we find that when the flow was strong to the southwest (SW), the cold, fresh, and low PPV anomalies were located in the uLSW depth range. When the colder, fresher low PPV water was found in the dLSW depth range, the velocity anomalies were positive and thus the SW flow was weaker. How does this relation translate into transport? The transport (per unit width) for the uLSW and dLSW layers can be expressed as the product of the layer thickness, $h$, and the velocity averaged within each layer, $v$ :

$$
T=v \cdot h
$$

Both layer thickness and velocity can be separated into a mean and a perturbation:

$$
\begin{aligned}
& v=\left(v^{\prime}+\bar{v}\right) \\
& h=\left(h^{\prime}+\bar{h}\right)
\end{aligned}
$$

Substituting into the transport expression and subtracting the mean, we find the variability in the transport, $T^{\prime}$, consists of three terms:

$$
T^{\prime}=(v \cdot h)^{\prime}=v^{\prime} \cdot \bar{h}+\bar{v} \cdot h^{\prime}+v^{\prime} \cdot h^{\prime}
$$

The first term in the right hand side of the equation represents changes in the transport due to the changes in the velocity, the second includes changes due to changing layer thickness, and the last is the non-linear term representing the part of the transport variability that is due to correlated changes in the velocity and layer thickness. Because we are interested in the low frequency signals that are associated with changes 


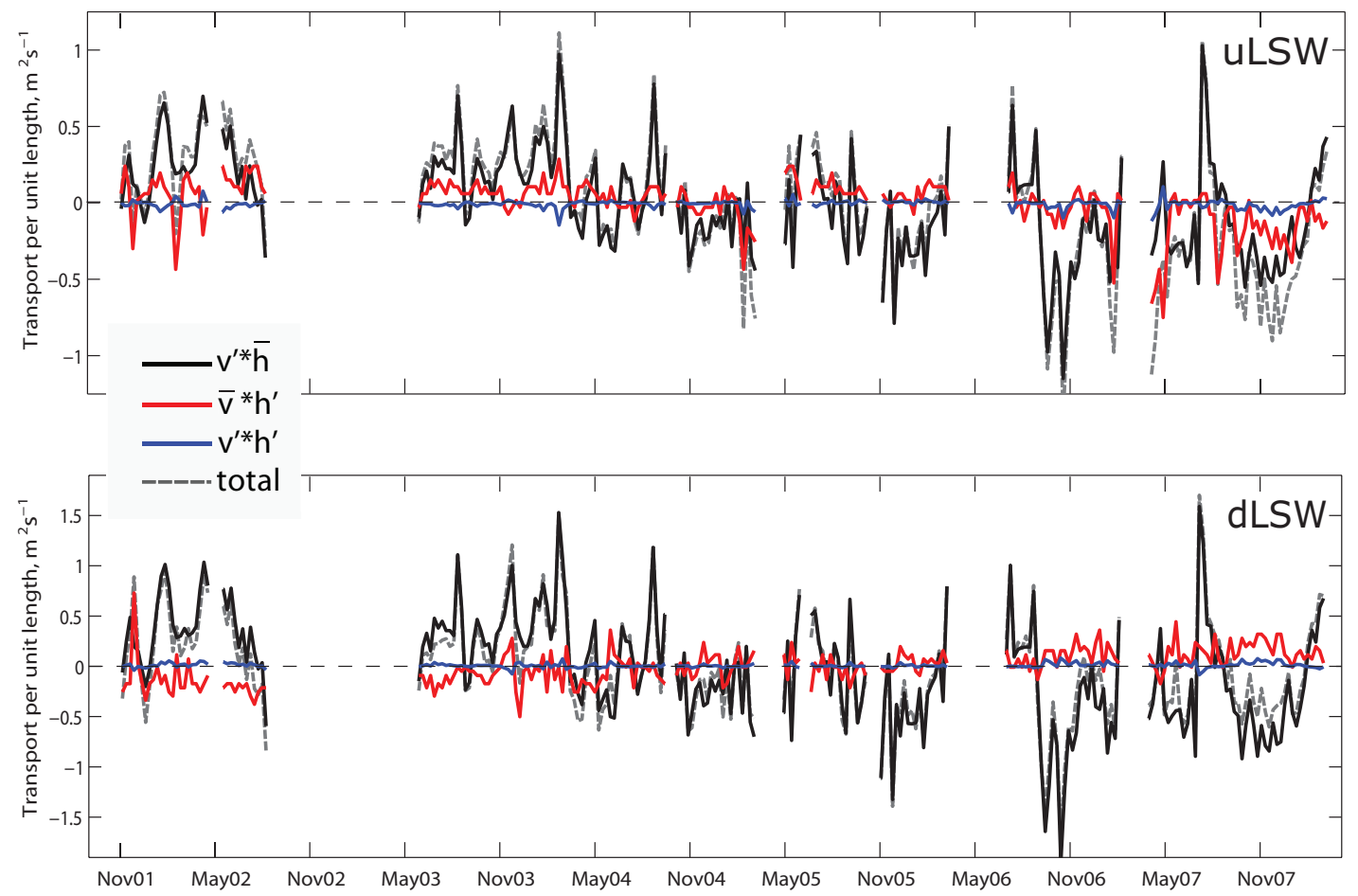

Figure 3-6: Transport decomposition. uLSW shown in the upper panel and dLSW in the lower. Values are transport per unit length, $m^{2} s^{-1}$. Negative meaning downstream in the direction of DWBC (southwestward).

in the density field we replace $v^{\prime}$ by the part that is captured in the EOF analysis in the previous section. Using the neutral density boundaries for the water masses shown in Table 3.3 we calculated the transports time series for the uLSW and dLSW (figure 3-6).

Table 3.3: Neutral density surfaces, $\gamma_{n}$, used in defining the water mass boundaries. Values shown in $\mathrm{kg} / \mathrm{m}^{3}$.

\begin{tabular}{ccc}
\hline \hline & UpperBoundary & Lower Boundary \\
\hline uLSW & 27.8 & 27.897 \\
dLSW & 27.897 & 27.983 \\
OW & 27.983 & 28.066 \\
\hline
\end{tabular}

The contribution from the non-linear term to the transport is negligible, although of opposite sign for the two water masses. Variations in the transport are, for the 
Temporal evolution of layer thickness

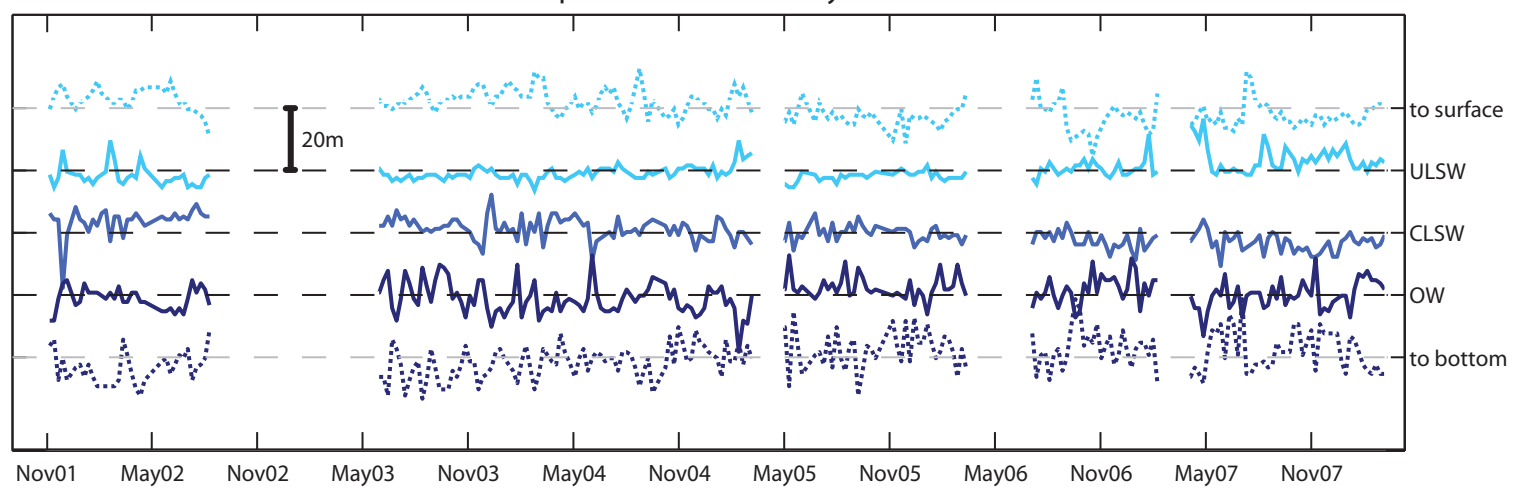

Figure 3-7: Temporal evolution of layer thickness, for the uLSW, dLSW and OW. The uppermost and lowermost lines are the integral between the upper boundary of the uLSW and the surface, and the lower boundary of the OW and the bottom.

most part, due to variations in the velocity field. This is in part due to the fact that $\bar{h}>h^{\prime}$ and $\bar{v} \approx v^{\prime}$. The evolution of the total transport for both layers is similar, with increasing southward transports toward the end of the record. The distribution between the terms is different. In the uLSW the contribution of layer thickness and velocity have the same sign, they are both positive or both negative, while in the dLSW these two terms are opposing. When the southward dLSW flow accelerates, the layer thickness decreases. Based on the term $\bar{v} \cdot h^{\prime}$ alone, we see some degree of compensation between the transport of the two LSW types, as found by Rhein et al. (2007) in the Labrador Sea. But this compensation at W3 is done entirely by the density field. This is clearly seen if we take a step back and look at the evolution of the layer thickness (see figure 3-7). As expected, when the uLSW layer expands the dLSW contracts. The amplitudes of the trends are similar, and they largely cancel out when added together. This compensation is, as we said, partial because transport changes are due to both changes in the layer thickness and changes in the velocity field. Thus, changing thickness of opposite sign in the two classes of LSW can still be associated with a net transport change of the same sign (dashed line in 3-6) caused by velocity changes.

Figure 3-7 also reveals another interesting aspect of the variability in this area. Independently of the compensation occurring at mid depth in the LSW, we find that 
the isopycnals across the entire water column are rising with time. This has resulted in the thinning of the uppermost layer (surface to $\gamma_{n}=27.8 \mathrm{~kg} / \mathrm{m}^{3}$ ) and the expansion the bottom most layer $\left(\gamma_{n}=28.066 \mathrm{~kg} / \mathrm{m}^{3}\right.$ to bottom). The rising of the isopycnals is consistent with the overall cooling seen in figure 3-2.

\subsection{Water mass variability}

Some of the variability observed in figure 3-2 is related to the heaving of the isopycnals as we have just discussed, and not to real water mass changes. To separate these two sources of stratification variability and investigate changes in the water masses, we transform the vertical coordinate to neutral density. Using the mean density profile as a reference, we defined the increments in the density axis, $\gamma_{n}^{i}$, to be volume conserving on average. In one dimension, this is equivalent to say that the mean vertical distance between all adjacent density surfaces is the same, $\delta Z_{\gamma_{n}^{i}}=$ constant, not the density interval itself, $\delta \gamma_{n}^{i} \neq$ constant.

We consider two different approaches to investigate water mass variability in the isopycnal coordinate system. First, we look at temperature and salinity changes in a water mass bounded by two neutral density surfaces using the same layer definitions used in the transport calculation (see Table 3.3). Second, we look at changes in the density of a particular water mass. The properties of the water masses formed by convection in the Labrador Sea vary on interannual time scales, and they do so not only by changing their temperature and salinity but their volume and density as well (Yashayaev (2007)). To explore these changes in the density of the different water masses we will define them based on their PPV signature.

\subsection{1 $\theta / \mathrm{S}$ variability (fixed- $\gamma_{n}$ range).}

To explore interannual $\theta / \mathrm{S}$ changes in the water masses, we calculated Novemberto-November means (to maximize data usage) for the 5.5-year record. The irregular distribution of temporal gaps through the record, in particular the 295-day gap 


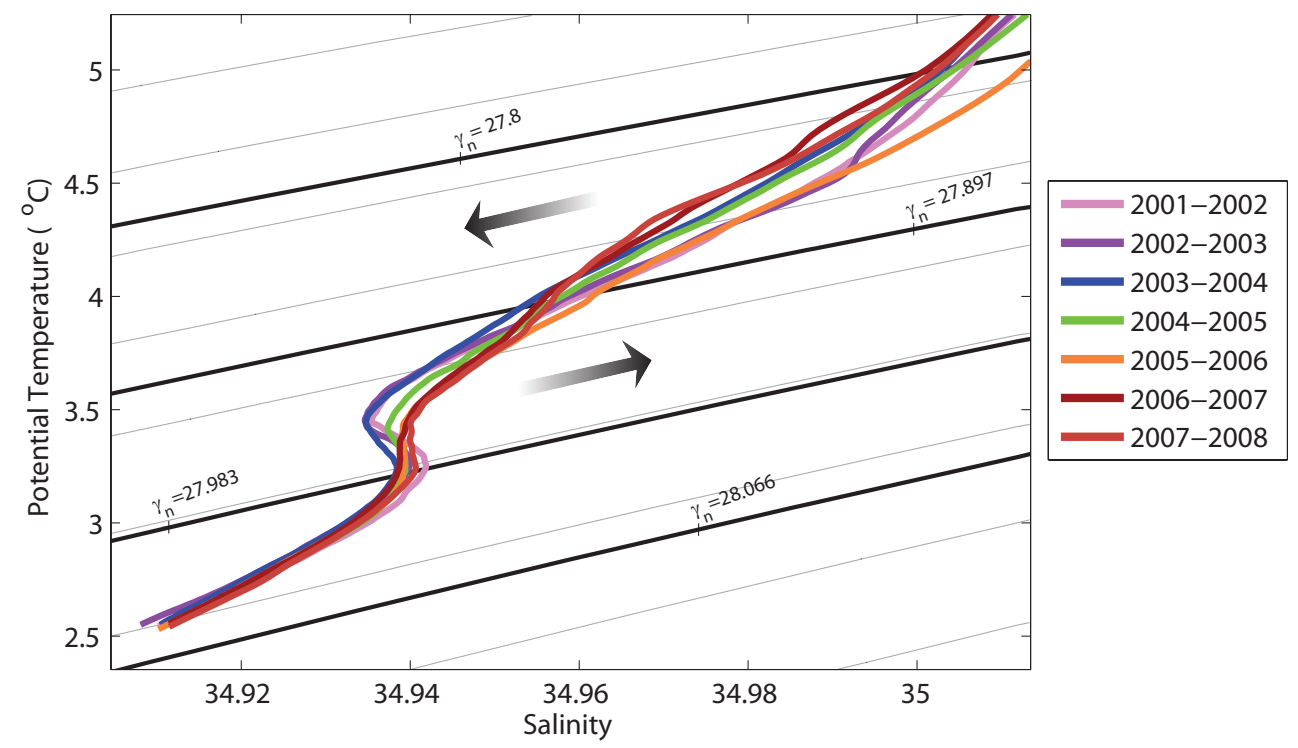

Figure 3-8: $\theta / \mathrm{S}$ diagram. The gray contours are the isopycnals, in black are the boundaries of the uLSW, dLSW and OW. The arrows indicate the transformation in the dLSW and uLSW layers.

between the first and second deployments, can cause some differences between the annual mean estimates that are not real but the result of averaging different amounts of data. This is also true for the last time interval, which includes only of 6 months of data. We must be careful then in interpreting these results.

The $\theta / \mathrm{S}$ diagram of the annually averaged data (figure 3-8) shows that within the dLSW layer, the water becomes progressively warmer (by about $2^{\circ} \mathrm{C}$ ) and saltier (by about 0.01 ) throughout the observational period. The salinity minimum that characterizes dLSW is slightly denser than the mean layer density, suggesting the real boundary of the water mass may be lower than the $\gamma_{n}=27.983 \mathrm{~kg} / \mathrm{m}^{3}$. In time, the minimum erodes and it has almost disappeared by the end of the record. The same evolution was seen by Yashayaev (2007) in the central Labrador Sea. In the uLSW the situation is the opposite. The water cools and becomes fresher with time. There is no sign of the salinity minimum that characterizes this water mass in the Labrador Sea (Stramma et al. (2004)), presumably due to the mixing with the warmer and more saline surrounding water (Pickart et al. (1996)). The $\theta / \mathrm{S}$ curve for the 2005-2006 period is somewhat anomalous. We believe this is due to a warm core 
ring event in the Spring 2006, whose effect on the water masses was not completely removed from the record.

\subsubsection{Density variability in the water masses.}

The layer thicknesses of the uLSW and dLSW that one infers from the vertical distribution of positive and negative PPV anomalies in figure 3-2 is more variable than what is indicated by the fixed-density range layer definitions (figure 3-7). These larger fluctuations in layer thickness are consistent with the evolution of the LSW thickness in the Labrador Sea shown by Yashayaev (2007). The density of the LSW layer that is produced every year by convection is variable as well, and thus representing the different types of LSW by two fixed density surfaces can be misleading (e.g. Stramma et al. (2004) and Yashayaev et al. (2007)). For this reason, we will explore density variability within the water masses using PPV to define the layers instead of fixed $\gamma_{n}$ values.

To identify the water masses we used a scaled form of potential vorticity in density coordinates, namely potential thickness. To obtain an expression for potential thickness, $\tilde{q}_{\gamma_{n}}$, we start by exchanging density and pressure in the definition of PPV in Z-coordinates, $q$, and scaling by pressure:

$$
\begin{gathered}
q \propto-\frac{1}{\rho} \frac{d \rho}{d z} \\
q_{\gamma_{n}} \propto \frac{d z}{d \gamma_{n}}
\end{gathered}
$$

Neutral density, $\gamma_{n}$, has replaced density, $\rho$, in the isopycnal coordinate system. Because the density coordinate transformation is volume conserving (density intervals are defined so that they contained the same volume of water), $d \gamma_{n}$ in the denominator of the second expression is not constant, thus interpreting $q_{\gamma_{n}}$ can be complicated. 


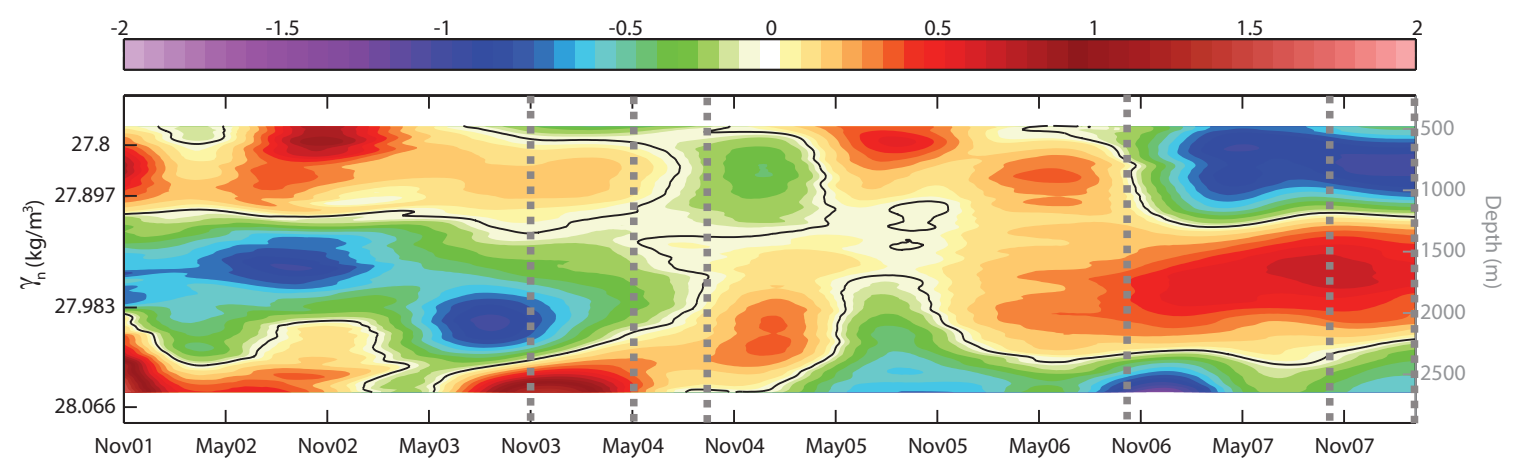

Figure 3-9: Layer stretching anomaly, $\tilde{q}_{\gamma_{n}}^{\prime}$, in decibars calculated from the smoothed density field. The right axis shows the depth of the corresponding $\gamma_{n}$ in the mean. The black line is the zero anomaly contour, $\tilde{q}_{\gamma_{n}}^{\prime}=0$. The gray dashed lines indicate dates of the hydrographic sections used in 3.6.

For that reason we normalized $q_{\gamma_{n}}$ by $d \gamma_{n}$, and define a new variable:

$$
\tilde{q}_{\gamma_{n}}=q_{\gamma_{n}} \cdot d \gamma_{n}
$$

$\tilde{q}_{\gamma_{n}}$ is a measure of the stretching, in meters, of the density layers (in the infinitesimal sense). In the mean, by definition $\tilde{q}_{\gamma_{n}}=$ constant.

Similar to its Z-coordinate equivalent, due its derivative nature, $\tilde{q}_{\gamma_{n}}$ is very noisy and unless the density field is smoothed, that noise dominates the interannual signals. Individual profiles were smoothed with a $250 \mathrm{~m}$ box-car filter, and the top and bottom $150 \mathrm{~m}$ of the water column eliminated to avoid edge effects. The resulting vertically smoothed profiles were low-pass filtered with 1-year digital Butterworth filter. We choose a year filter cutoff because this is the time scale of the fluctuations in the dense water formation process. Gaps in the data were filled by linear interpolation (none of the gaps are longer than the filter's window so linearly interpolating did not introduce any additional data). Once the smooth $\tilde{q}_{\gamma_{n}}$ was calculated and its mean removed, we used potential thickness anomalies, time-mean removed, (see figure 3-9) to investigate density changes in the LSW.

In the time series of potential thickness anomalies, recently ventilated waters appear as negative features (less stratified than the mean) coherent (vertically) in 
space as well as in time. We take the zero anomaly contour, $\tilde{q}_{\gamma_{n}}^{\prime}=0$, as the boundary between the layers. Features whose depth core is located between 500 and $1000 \mathrm{~m}$ trace the evolution of uLSW, while features between 1000 and 2500m are dLSW. Below is the OW. These boundaries are approximate, not always clearly defined and can sometimes overlap. The properties at the $\tilde{q}_{\gamma_{n}}^{\prime}$ minimum and the thickness of the layers bounded by the zero contour experience large variations. The core of newly ventilated dLSW deepens by about 500m, from 1500m where is found in 2001 to approximately $2000 \mathrm{~m}$ in 2003. This change in depth is accompanied by the corresponding change in density, going from $\gamma_{n}=27.95 \mathrm{~kg} / \mathrm{m}^{3}$ in 2001 to $\gamma_{n}=28 \mathrm{~kg} / \mathrm{m}^{3}$ in 2003 . The latter value is larger than the lower boundary of the dLSW used in the previous transport calculation. This suggests that we could at times be mis-assigning about $25 \%$ of the dLSW transport to OW. The thickness of the uLSW as seen in figure 3-9 is also larger than the thickness of the layer based on the fixed density boundaries. So its transport could be underestimated as well.

The evolution of PPV in Z-coordinates shown in Figure 3-2 suggested that the two types of LSW at W3 alternate throughout the record. The same is true in density coordinates, however the transition between the dLSW and uLSW is much more abrupt than seen in Z-coordinates, indicating that the PPV changes during the transition were caused by the isopycnal heaving rather than changes within a density class. Both of these views, $\mathrm{Z}$ and density coordinates, are in good agreement with the conditions observed in the Labrador Sea half a decade earlier. According to Yashayaev (2007), deep convection in the Labrador Sea stopped in 1994, at which time the LSW was its densest and most voluminous. This very dense LSW was observed at Line W in 2003, implying a spreading time for the dLSW of 9 years. Once the strong convection ceased in the Labrador Basin, the weaker convection responsible for the formation of uLSW did not start until the winter of 2000. By 2002 the uLSW reached its maximum thickness in the Labrador Sea, and only 5 to 6 years later, in 2007, this thick layer of uLSW was observed at Line W.

One last interesting aspect seen in the evolution of layer stretching anomalies is the 
out-of-phase relation found between OW and dLSW. Because these water masses are formed in different locations, the formation of the one does not impact the formation of the other, as is the case for upper and deep LSW. However, it is possible that as the dLSW layer expands, it occupies the upper part of the OW depth range. The OW, whose lower boundary is practically at the bottom at W3, might then be forced offshore or takes an alternative equatorward route through the interior. This would also explain why the transition between OW and dLSW is so abrupt. As soon as the dLSW layer vanishes at W3, the OW layer develops again.

\subsection{The fate of the LSW}

Downstream from Line $\mathrm{W}$ as the DWBC approaches Cape Hatteras, it is forced to cross under the Gulf Stream in order to continue to flow south along the boundary. According to Bower and Hunt's (2000) float observations, the deeper layers in the DWBC manage to slide under the Gulf Stream whereas at intermediate depths the water recirculates back to the northeast in the deep Gulf Stream. The same behavior was observed by Pickart and Smethie (1993) for the deep layers. In addition, they found that little uLSW was found south of the cross-over, most of the shallow components of the DWBC were entrained by the Gulf Stream. In this section we use PPV, once again, to explore the recirculation and entrainment of recently ventilated LSW.

We constructed two composite sections, one for the dLSW-dominated period (pre2004), and one for the uLSW (post-2006). Each composite section was made up of data from 3 cruises. The data from each cruise were interpolated into a $10 \mathrm{~km} \mathrm{x} 50 \mathrm{~m}$ common grid and PPV anomalies for each period obtained as the difference between the 3-cruise composite sections and the average of all sections (figure 3-10).

During the post-2006 period, confined to the innermost $150 \mathrm{~km}$ of the section adjacent to the shelfbreak at the uLSW depth range, we find the distinct low PPV anomalies associated with the newly ventilated water. The bulk of the low PPV uLSW is however located within the southwestward flow in the upper DWBC/Slope Water, 

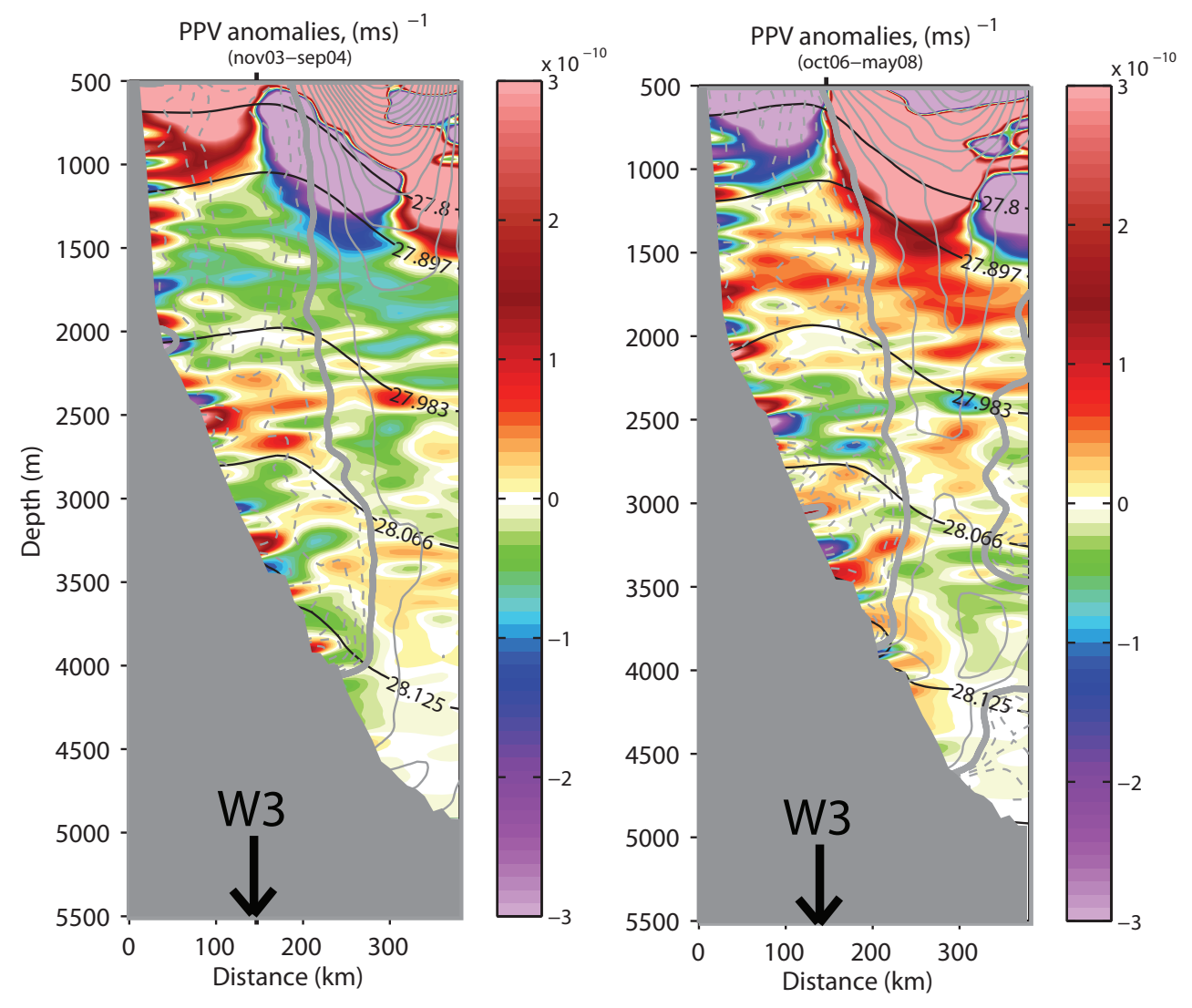

Figure 3-10: The color maps show PPV anomalies, superimposed are the neutral density contours corresponding to the uLSW, dLSW and OW boundaries in black, and downstream velocities in gray (solid lines are positive values, and negative dashed). On the left anomalies for the pre-2004 composite, and on the right anomalies for the post-2006 composite.

suggesting that by the time the recirculating uLSW reaches Line W, its signature has been diluted through mixing with the warmer, more saline, higher PPV Gulf Stream waters that occupy the same density range. Beneath the uLSW, negative PPV anomalies are found in the dLSW layer during the pre-2004 period. The dLSW layer, unlike the uLSW, dives under the lighter thermocline waters, and spreads offshore without obstruction. The recirculation of dLSW is clearly seen at Line W as the laterally coherent low PPV signal is advected by both the equatorward flowing DWBC and the poleward flow in the deep Gulf Stream.

Some other features that stand out in these PPV composite sections are the anomalies in the upper $1000 \mathrm{~m}$ caused by changes in the location of the Gulf Stream. 
These anomalies are large and positive when the Gulf Stream is north of its mean position; such is the case for the post-2006 composite, and large and negative when the stream is south of the mean, as in the pre-2004 composite. The relation between the position of the Gulf Stream and the variability in the water masses along the DWBC seen here is not coincidental and it will be discussed in Chapter 3.

\subsection{Summary and discussion}

In the present study we have explored changes in the properties of the LSW within the DWBC southeast of Cape Cod for the period of November 2001 to May 2008. The most significant of the changes we observed is in the PPV field. The PPV minimum (in the anomalies) that traces the core of the waters formed by convection in the Labrador and Irminger Basins, slowly migrated from a depth of $1500 \mathrm{~m}$, where dLSW is typically found, to 700m, typical of uLSW. Between 2001 and 2003, the PPV anomaly minimum was found deeper in the water column and it was more pronounced. After 2003 the PPV signal was more diffuse, and it was not until 2006 when the PPV minimum migrated to the uLSW level that it had a distinct signal again. The transition between the two types of LSW corresponds to a period when little or no convection was observed in the Labrador Basin (eg. Schott et al. (2004) and Yashayaev (2007)). Over time the water re-stratified and its signature as it propagated equatorward can be seen in the gradual rising of the isopycnals between 2003 and 2006. A description of the changes occurring in the newly ventilated LSW was obtained by working in isopycnal coordinates to remove the effects of isopycnal heaving. We found that during the intensification of the deep PPV minimum in the early part of the record, the dLSW layer was expanding, and the stratification within it decreasing. The layer reached maximum density and thickness values around 2003. After 2004, the stratification in the dLSW increased and the uLSW layer started to develop. By 2007 the dLSW thickness had reached a minimum, and the uLSW layer was fully developed. The evolution of the layer thickness and density of both 
types of LSW agrees remarkably well with that seen in the central Labrador Sea during the 90s (Yashayaev (2007)), albeit later in time. The time difference between the potential vorticity minima observed in the central Labrador Sea and at Line W suggests that the two types of LSW have different spreading rates. Anomalies in dLSW take approximately 9 years to propagate from the central Labrador Sea to Line $W$, which implies a spreading rate of approximately $1.5 \mathrm{~cm} / \mathrm{s}$. The uLSW in the other hand, spreads more rapidly, taking only 5 years to reach Line W. This translates into a spreading rate of approximately $2.5 \mathrm{~cm} / \mathrm{s}$. These spreading rates are in much better agreement with those estimated by Molinari et al. (1998) than with the earlier estimates by Smethie (1993). Molinari et al. (1998) analyzed tracer data from a series of hydrographic sections across the DWBC at $26.5^{\circ} \mathrm{N}$, and estimated a transit time for the LSW from the Labrador Sea of 10 years, which implies a spreading speed of $2.5 \mathrm{~cm} / \mathrm{s}$, similar to what is found here for the uLSW. Smethie (1993) inferred spreading rates from $\mathrm{CFC}$ inventories that are one order of magnitude lower than ours. His numbers range from $11-12$ years at $45^{\circ} \mathrm{N}$ to 18 years at $32^{\circ} \mathrm{N}$. However, those previous estimates were based on a limited number of bottle measurements, compared to our continuous mooring record, and the uncertainties associated with the exact arrival time of the signals could be large. In that regard, we believe our estimate might be more accurate, since we were able to observe the exact time of transition between the dLSW and the uLSW. The more recent LSW transit times by Smethie and Smith (2010), 6-7 years from the central Labrador Sea to Line W, are in much better agreement with our observations.

Along with the density and layer thickness adjustments we find that on average, the water at W3 became colder and fresher with time. However, within the dLSW depth range, this tendency was reversed. The water here became warmer and saltier over time similar to what was found by Yashayaev (2007) in the central Labrador Sea.

Simple inspection of these anomaly time series suggest that the variability in the velocity field is not closely coupled with the variability in the stratification. Fluctu- 
ations in the velocity field are more rapid than those seen in the hydrography. The joint EOF of the temperature, salinity and velocity anomaly fields revealed that the fraction of the velocity variability that correlates with water mass changes is rather small (about $10 \%$ of the full velocity variability), with amplitudes of just $0.5-1 \mathrm{~cm} / \mathrm{s}$. Nevertheless, the sense of the correlated part is such that when newly ventilated dLSW is exported (PPV anomaly is negative at the dLSW level), the mean flow, to the SW everywhere in the water column, is less. This is true everywhere except for the bottom $500 \mathrm{~m}$ where the sign of the velocity change is the opposite. At times when the negative PPV anomaly is in the uLSW, the mean SW flow is greater. These changes in the velocity field are consistent with the spreading rates inferred from the arrival of PPV and potential thickness anomalies to Line $\mathrm{W}$, with the uLSW spreading nearly twice as fast as the dLSW.

The in-phase/out-of-phase nature of the relationships between the density and velocity fields in the uLSW and dLSW captured by the EOF analysis results in a partial compensation of the transport (per unit width) of the two types of LSW at W3. Using fixed-density ranges to define both LSW types we find that $25 \%$ of the transport per unit width variability at W3 is due to changes in layer thickness. These changes in layer thickness are responsible for the compensation between uLSW and dLSW above mentioned. The remaining $75 \%$ is related to changes in the velocity averaged across the layer. In the uLSW, the contribution of the changing velocity term together with the increasing layer thickness results in an overall transport change of about $-0.15 \mathrm{~Sv}$, if the the pointwise changes hold over a total width of the flow of $100 \mathrm{~km}$. In the dLSW, some of the acceleration of the SW flow is canceled by the decreasing layer thickness, leading to a net transport change of -0.2Sv (again assuming $100 \mathrm{~km}$ width). These transport changes are small compare to the transport variability in the DWBC found by others. Bryden et al. (2005) found that the transport of the synoptic DWBC at $26.5^{\circ} \mathrm{N}$ ranges from 5 to $75 \mathrm{~Sv}$. Similar values, and more relevant to the work presented here, are reported by Toole et al. (2010) using the full Line W moored array data for the period of 2004-2008. But recall, we are using here the EOF 
velocity mode that is correlated with PPV changes that only represents $10 \%$ of the full DWBC velocity variability. If we used the full velocity variability (typical changes of $\pm 15 \mathrm{~cm} / \mathrm{s})$, the transport variability we obtained is one order of magnitude larger $(-1.5 \mathrm{~Sv}$ for the uLSW and $-2 \mathrm{~Sv}$ for the dLSW), comparable to the amplitude of the response of the LSW transport to changes in the MOC found by Böning et al. (2006) but still smaller to what is found by Bryden et al. (2005) and Toole et al. (2010). The latter found that transport variations in the DWBC are dominated by width and velocity fluctuations, rather than by changes in the layer thickness. Because of the one-dimensional nature of the W3 observations the effect of changes in the lateral extension of the DWBC cannot be considered here. This and other aspects of the variability in the flow of the DWBC associated with lateral changes in the DWBC will be addressed in Chapter 4 .

With regard to the fate of the LSW downstream from Line W, our observations suggest that an important fraction of the LSW that is transported by the DWBC at $69^{\circ} \mathrm{W}$ recirculates back into the mid-Atlantic Bight upon reaching Cape Hatteras. The recirculating dLSW slides under the upper Gulf Stream waters, spreading offshore relatively unobstructed. The recirculation of the uLSW as seen by Bower and Hunt (2000) float trajectories occurs within a small region north of the Gulf Stream. However, by the time the recirculating uLSW reaches Line W, its signature can no longer be distinguished from the Gulf Stream, most likely due to the entrainment of large amounts of much warmer, more saline and higher PPV waters as it flows back into the Slope Water. Evidence of this recirculation and entrainment of upper DWBC water was found by Pickart and Smethie (1993).

The work presented here suggests that the DWBC is an active pathway for the export of LSW, contrary to what was inferred from float trajectories deployed in the Labrador Sea (Fischer and Schott (2002) and Bower et al. (2009)). This apparent contradiction between the Lagrangian view provided by the floats and the Eulerian description of the variability based on the evolution of the PPV, can be in part explained by the different nature of the processes that govern the motion of the floats 
versus the spreading of a water mass inferred from a tracer. The motion of the floats is a purely advective process in which the displacements of a water parcel, whose properties are changing due to mixing, are determined by the instantaneous velocity field. The export of a tracer, on the other hand, is the result of an advective-diffusive balance. In this balance, the advection is due to mean flow, or the slowly varying part of it, while the integrated effect of the eddy variability is included in the diffusion term. The DWBC contributes to the advective term in this balance. This was shown by Getzlaff et al. (2006) simulated float trajectories. When the mean circulation was considered, $90 \%$ of the floats that were deployed in the Labrador Sea and reached the subtropics did it via the classical path in the DWBC, whereas only $60 \%$ did it when the variability was used instead. In addition, two other aspects of the spreading of the LSW may be important in explaining the differences between Bower et al. (2009) floats and our mooring observations. First, Talley and McCartney (1982) found that the core depth of the LSW experiences a drop of about 500m as it turns around the Grand Banks. Bower et al. (2009) floats being isobaric, are unable to capture this change. Second, the density of the water that is formed in the Labrador Sea changes from year to year, and as we showed here, these changes in density are associated with large changes in the core depth of the LSW. This temporal variability was not accounted for by the floats, that were deployed at the same location and depth during the entire Bower et al. study period.

Despite the evidence of LSW along the DWBC, its contribution to changes in the Meridional Overturning Circulation (MOC) is nevertheless debatable. The response observed in the subtropics to the changing forcing in the Labrador Sea is a combination of layer thickness changes, density changes and changes in the velocity. The latter represents a small part of the variability of the velocity field, and therefore the transport. The changes in the transport we obtained by applying the point-wise estimates over the typical width of the DWBC, are small compared to DWBC transport variability observed by Toole et al. (2010) with the full moored Line W moored array dateset. In the Labrador Sea, the ocean's response to the atmospheric forcing hap- 
pens also through changes in the horizontal circulation (Häkkinen and Rhines (2009)) as well and changes in the formation rates and properties of the LSW (eg. Clarke and Gascard (1983)). The relative contribution of the latter, the one represented here by PPV changes, to the MOC was found to be small by Pickart and Spall (2007) when compared to the contribution of the horizontal circulation. Our observations at W3 are consistent with Pickart and Spall (2007) findings, but lack information about the lateral structure of the DWBC which could significantly alter the results presented here. 


\section{Chapter 4}

\section{Variability in the DWBC, local vs. remote forcing}

\subsection{Introduction}

Dense water formation in the subpolar and subarctic basins has traditionally been thought to have a large impact in the Atlantic Meridional Overturning Circulation (AMOC). In Stommel and Arons' (1960) theory for the abyssal circulation of the ocean, localized sinking at high latitudes is balanced by homogeneously distributed upwelling. The circulation in the interior, a poleward flow driven by the upwelling, is closed by a southward flowing current located along the western boundary due to vorticity constraints. The strength of the boundary current is directly related to the strength of the sinking, in that the boundary current is the only part of the circulation responsible for exporting dense water away from the formation sites. Although the Stommel-Arons theory was formulated for the steady circulation of the ocean, one might expect that changes in the dense water formation that occur over long time scales, as is the case for the dense water formed in the northern North Atlantic, will induce changes in the transport of the Deep Western Boundary Current (DWBC) and in the AMOC in general.

The properties as well as the formation rates of the dense water originating in 
the Labrador Sea, one of the most studied convection sites, are known to experience large fluctuations at interannual and longer time scales (eg. Lazier (1980), Talley and McCartney (1982) and Yashayaev (2007)). The large heat fluxes (from the ocean to the atmosphere) that occur in the Labrador Sea during winter time are partly responsible for the densification of the surface water and the consequent overturn. However, as was noted by Clarke and Gascard (1983), fresh water fluxes and changes in the cyclonic circulation of the subpolar gyre can also play a crucial role. The deepest and strongest ever observed convection in the Labrador Sea occurred during the late 80's and early 90's (Yashayaev,). During this period, as well as during previous enhanced convection events, the North Atlantic Oscillation index (NAO) was large and positive. During the late 90's, as the NAO entered a negative phase, convection ceased in the Labrador Sea, and it was not until the early 2000's that convection re-started, but reaching to much shallower depths than the previous decade. This relation between the NAO and the LSW variability was clear in Curry and McCartney (2001) LSW temperature record, with the temperature evolution of the Labrador Sea mirroring the NAO-index.

The return of deep convection to the Labrador Sea in the winter of 2007-2008 (Våge et al. (2009) and Yashayaev and Loder (2009)) suggests a more complex relation between atmospheric forcing and convection than previous studies have shown. This recent reestablishment of the deep convection was described as "surprising" by Våge et al. (2009) due to the atypical conditions existing in the Labrador Sea during that winter. According to these authors, the relation between atmospheric forcing and the strength of convection is highly complex, and involves several other factors such as sea-ice distribution and storm tracks. Hence the chances of successfully predicting future deep convection events are small.

The formation rates and properties of the Overflow Water, including Denmark Strait (DSOW) and Iceland-Scotland Overflow Water (ISOW) are also subject to interannual fluctuations (Macrander et al. (2005)). However, the relation between changes in the OW and changes in the atmospheric forcing has the opposite sense to 
that in the Labrador Sea. During high NAO periods, when convection is maximum in the Labrador Sea, the Nordic Seas grow progressively warmer and more saline due to weakened deep convection that in turn may be caused by an increase in the influx of low-density water of Arctic origin (Dickson et al. (1996)).

Regardless of what causes the observed variability in convection in the northern North Atlantic, whether or not these changes in dense water formation can cause changes in the MOC, remains unclear at this point. The lack of long term measurements, the different definitions of the overturning streamfunction, and the variety of model configurations make the comparison of different studies rather challenging. Of the two major constituents of the NADW, the LSW and the OW, the relation between the latter and the MOC inferred from observations and models (and between models themselves) appears more robust. In Gerdes and Koberle's (1995) model of the North Atlantic, an increase in the production of OW leads to a stronger DWBC and more vigorous meridional overturning. This result is in good agreement with Koltermann et al.'s (1999) findings. Their analysis of hydrographic data from a series of repeated sections in the North Atlantic suggested that the dominant driver of the MOC is the DSOW. Variations in the production of the LSW did not appear to have any effect on the strength of the MOC. This same conclusion was reached by Böning et al. (1996) and Kohl and Stammer (2008) modeling experiments as well as by Pickart and Spall (2007) from both model and observations. It is also supported by Schott et al.'s (2006) temperature and velocity moored observations along the DWBC, that showed a warming trend at the depth of the LSW with no significant long term changes in the corresponding transports. The lack of a LSW contribution to the MOC is however at odds with the meridional overturning streamfunction estimates by Talley et al. (2003). Similarly, Mauritzen and Häkkinen (1999) found that a reduction in the formation of LSW can cause a decrease in the strength of the MOC. The increase in the MOC strength that follows periods of high NAO in Eden and Willebrand (2001) modeling study further supports the role of the LSW in the AMOC, since high NAO is related to enhanced production of LSW. 
Many of the observations described above are either based on a limited number of observations (thus strongly influenced by the ocean's eddy variability and not necessarily representative of the long term changes) or have poor vertical resolution (and so cannot properly resolve the vertical structure of the changes associated with the production of the different constituents of the NADW). The work presented here is novel in that is based on a four-year record of temperature, salinity and direct horizontal velocity measurements with both high temporal and spatial resolution. In this study we explore the variability in the water masses that are formed by convection at high latitudes as they are exported southward into the subtropical domain along the western boundary, and describe the changes in the circulation in the western North Atlantic that are associated with them. The remainder of the chapter is organized as follows: in the first section we introduce the data set and the mapping technique employed to grid the data. In section two we discuss the mean circulation and develop a filter to eliminate Gulf Stream rings and meanders from the array, which can dominate the low frequency signals in the DWBC. In section three we look at the energetics of the flow, with special attention to the high frequency signals. In section four we investigate the low frequency changes in the circulation that correlate with changes in the water mass distribution. In section five we present transport estimates for the water masses that are well-resolved by the array, and finally in section six, before the conclusion, we discuss an abrupt change in the circulation and hydrographic properties in the array that occured in the winter of 2007-2008 towards the end of the record.

\subsection{The data set}

\subsubsection{The Line $\mathrm{W}$ array}

The Line $\mathrm{W}$ record consists of a total of 5 moorings spanning the measurement period May 2004 to April 2008. The array was located along the continental slope in the western North Atlantic at approximately $69^{\circ} \mathrm{W} 40^{\circ} \mathrm{N}$ (see location in figure 4-1). The 


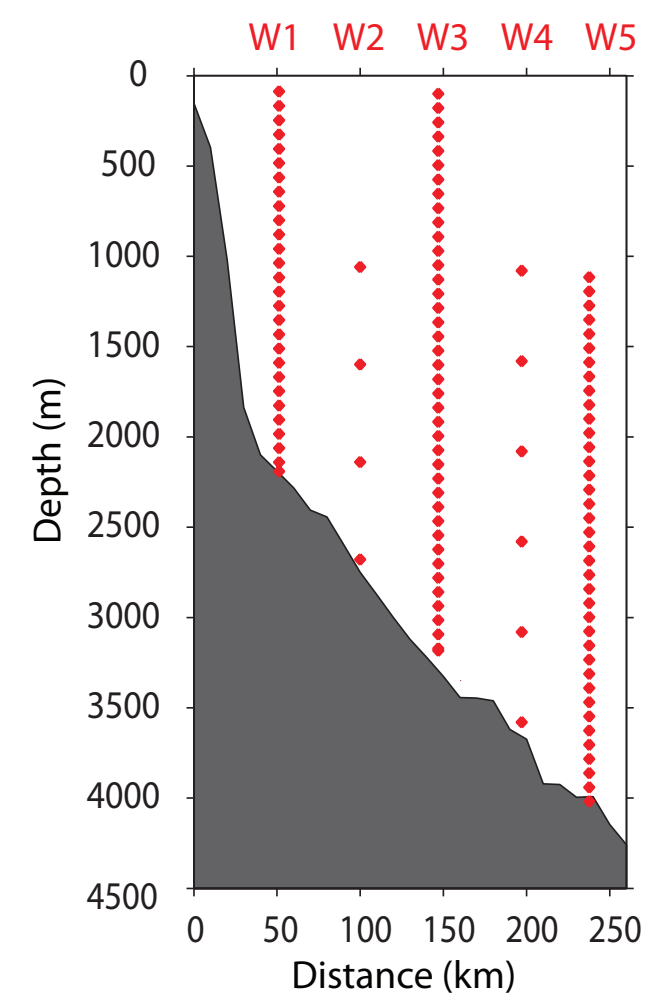

Figure 4-1: On the left map showing the location of the Line $W$ array in the continental slope, indicated by the red dots. The dashed-line arrow indicates the mean southwestward flow in the DWBC, and the solid-line the Gulf Stream. On the right is a schematic of the mooring set up. Moorings W1, W3 and W5 correspond to the MMP moorings. The dots in moorings W2 and W4 represent the approximate location of the current meters. For the exact depths see table 4.1.

orientation of the array is $151^{\circ}$ (from the North direction), approximately perpendicular to the local bathymetry in the area and the direction of the mean southwestward flow in the DWBC. The along-isobath direction is the nominal downstream direction that will be used throughout this chapter. The mean Gulf Stream intersects the array not quite perpendicularly, due to its slightly more zonal path.

The array is a mixture of profiling moorings (denoted W1, W3 and W5 in figure 4-1), equipped with McLane Mooring Profilers (MMP) measuring temperature, pressure, conductivity and horizontal velocity, and fixed-depth sensor moorings (denoted W2 and W4), equipped with Vector Averaging Current Meters (VACM) and MicroCAT's (temperature and conductivity sensors). The MMP moorings were also 
equipped with VACM and Microcats at the top and/or bottom of the MMP range. The record consists of four 1-year deployments for the MMP moorings (higher battery demands required more frequent turn-around), and two 2-year deployments in the case of the fixed-depth sensor moorings.

The MMP data were binned during processing to a vertical resolution of $2 \mathrm{db}$, with the shallower moorings, W1 and W3, covering the entire water column range (from $100 \mathrm{~m}$ to the bottom approximately), and the deepest of the mooring, W5 that sat near the Gulf Stream's North Wall, sampling between 1000m and the bottom (ca. $4500 \mathrm{~m}$ at this location; figure 4-1). The MMPs were programmed to sample in bursts with each burst separated by 5 days and consisting of four one-way profiles. Averaging all four profiles effectively removes both the tidal and inertial peaks (Silverthorne and Toole (2009)). VACM's recorded vector averaged velocity estimates at 30 minute interval while the the MicroCAT's were programmed to sample every 15 minutes. The records were subsequently filtered with a 2-day low-pass filter and subsampled daily. A summary of the instrument depths for all moorings is provided in table 4.1.

Table 4.1: Mooring setup.

\begin{tabular}{cccc}
\hline \hline Moor. No. & \multicolumn{3}{c}{ Instrument type and depth } \\
\hline W1 & MMP: 60-2150m & VACM: 60, 2150m & T/S: 60, 2150m \\
W2 & & VACM: 1030, 1630, & T/S: 1030, 1330, \\
& & 2220, 2680m & $1630,1930,2225$, \\
& & & $2320,2410,2540$, \\
W3 & \multirow{2}{*}{ MMP: 60-3150m } & VACM: 60, 2150m & T/S: 60, 2150m \\
& & VACM: 1040, 1640, & T/S: 1040, 1340, \\
W4 & & 2240, 2690, 3240, & $1640,1940,2240$, \\
& & $3570 \mathrm{~m}$ & $2690,2930,3140$, \\
& & & $3230,3320,3430$, \\
W5 & MMP: 1000-4050m & VACM: $1000,4100 \mathrm{~m}$ & T/S: $1000,4100 \mathrm{~m}$ \\
\hline
\end{tabular}

For a detailed discussion of the instrument setup and data processing, the reader is referred to Toole et al. (2010). 


\subsubsection{Constructing an objectively mapped section}

The Line $\mathrm{W}$ array consists of both moorings with high vertical resolution and low temporal resolution (W1, W3 and W5) and moorings with high temporal resolution and low vertical resolution (W2 and W4). To take advantage of both, we used a three dimensional (horizontal, vertical and time) objective mapping routine to grid the observations into $10 \mathrm{~km}$ x $20 \mathrm{~m}$ x daily resolution dataset. Next is a brief description of the method. For a detailed derivation of the interpolation technique, see Wunsch (2006); the same notation is used here.

We want to estimate the value of the variable $\mathbf{x}$ at a series of locations $\hat{\mathbf{r}}$, as a function of both time and space, provided some information about its mean, $\langle\mathbf{x}\rangle^{1}$, and covariance, $\mathbf{R}_{x x}$.

$$
\begin{array}{r}
\langle\mathbf{x}\rangle=0 \\
\mathbf{R}_{x x}=\left\langle\mathbf{x}(\hat{r}) \mathbf{x}(\hat{r})^{T}\right\rangle
\end{array}
$$

To do so we have observed the value of the variable $\mathbf{x}$ at a set of locations $\mathbf{r}$. The observations are denoted $\mathbf{y}(\mathbf{r})$, for which we also have some knowledge of their mean and covariance.

$$
\begin{array}{r}
\langle\mathbf{y}\rangle=0 \\
\mathbf{R}_{y y}(\mathbf{r}, \mathbf{r})=\left\langle\mathbf{y}(\mathbf{r}) \mathbf{y}(\mathbf{r})^{T}\right\rangle \\
\mathbf{R}_{x y}(\hat{\mathbf{r}}, \mathbf{r})=\left\langle\mathbf{x}(\hat{\mathbf{r}}) \mathbf{y}(\mathbf{r})^{T}\right\rangle
\end{array}
$$

\footnotetext{
${ }^{1}$ The mean of the variable being mapped does not need to be zero, as is the case in our temperature, salinity and velocity observations. In practice, $\langle\mathbf{x}\rangle=0$ is achieved by simply removing the mean from the observations, $\langle\mathbf{y}\rangle$, prior to the mapping.
} 
The observations contain noise

$$
\mathbf{y}(\mathbf{r})=\mathbf{x}(\mathbf{r})+\mathbf{n}(\mathbf{r})
$$

where $\mathbf{x}$ is the noise-free value of the variable at locations $\mathbf{r}$ where the observations were taken. Our goal is to estimate the value of $\mathbf{x}$ at a new set of locations $\hat{\mathbf{r}}$, denoted $\tilde{\mathbf{x}}(\hat{\mathbf{r}})$ (the tilde stands for estimate), that are as close as possible to the real value, denoted $\mathbf{x}(\hat{\mathbf{r}})$. That is, we want to minimize the error function $\mathbf{P}$ defined as follows:

$$
\mathbf{P}(\hat{\mathbf{r}}, \hat{\mathbf{r}})=\left\langle(\tilde{\mathbf{x}}(\hat{\mathbf{r}})-\mathbf{x}(\hat{\mathbf{r}}))(\tilde{\mathbf{x}}(\hat{\mathbf{r}})-\mathbf{x}(\hat{\mathbf{r}}))^{T}\right\rangle
$$

We are going to write the estimates at $\hat{\mathbf{r}}$ as a linear combination of the observations at $\mathbf{r}$ with coefficients $\mathbf{B}(\hat{\mathbf{r}}, \mathbf{r})$.

$$
\tilde{\mathbf{x}}(\hat{\mathbf{r}})=\mathbf{B}(\hat{\mathbf{r}}, \mathbf{r}) \mathbf{y}(\mathbf{r})
$$

Substituting this expression for the estimates $\tilde{\mathbf{x}}(\hat{\mathbf{r}})$ into the error $\mathbf{P}$ we obtain:

$$
\mathbf{P}(\hat{\mathbf{r}}, \hat{\mathbf{r}})=\left\langle(\mathbf{B}(\hat{\mathbf{r}}, \mathbf{r}) \mathbf{y}(\mathbf{r})-\mathbf{x}(\hat{\mathbf{r}}))(\mathbf{B}(\hat{\mathbf{r}}, \mathbf{r}) \mathbf{y}(\mathbf{r})-\mathbf{x}(\hat{\mathbf{r}}))^{T}\right\rangle
$$

Introducing the covariance matrices for the observations, $\mathbf{R}_{y y}$, for the variable at the target positions, $\mathbf{R}_{x x}$, and for the observations and variable, $\mathbf{R}_{x y}$, and rearranging we obtain:

$\mathbf{P}(\hat{\mathbf{r}}, \hat{\mathbf{r}})=\mathbf{B}(\hat{\mathbf{r}}, \mathbf{r}) \mathbf{R}_{y y}(\mathbf{r}, \mathbf{r}) \mathbf{B}(\hat{\mathbf{r}}, \mathbf{r})^{T}-\mathbf{R}_{x y}(\hat{\mathbf{r}}, \mathbf{r}) \mathbf{B}(\hat{\mathbf{r}}, \mathbf{r})^{T}-\mathbf{B}(\hat{\mathbf{r}}, \mathbf{r}) \mathbf{R}_{x y}(\hat{\mathbf{r}}, \mathbf{r})^{T}+\mathbf{R}_{x x}(\hat{\mathbf{r}}, \hat{\mathbf{r}})$

Minimizing $\mathbf{P}$, we can solve for the coefficients $\mathbf{B}$ :

$$
\mathbf{B}(\hat{\mathbf{r}}, \mathbf{r})=\mathbf{R}_{x y}(\hat{\mathbf{r}}, \mathbf{r}) \mathbf{R}_{y y}(\mathbf{r}, \mathbf{r})^{-1}
$$


Rewriting the observations as the variable plus the noise

$$
\mathbf{R}_{y y}(\mathbf{r}, \mathbf{r})=(\mathbf{x}(\mathbf{r})+\mathbf{n}(\mathbf{r}))(\mathbf{x}(\mathbf{r})+\mathbf{n}(\mathbf{r}))^{T}
$$

and assuming the data and noise are uncorrelated:

$$
\mathbf{R}_{y y}(\mathbf{r}, \mathbf{r})=\mathbf{R}_{x x}(\mathbf{r}, \mathbf{r})+\mathbf{R}_{n n}(\mathbf{r}, \mathbf{r})
$$

we arrive finally at an expression for the estimates:

$$
\tilde{\mathbf{x}}(\hat{\mathbf{r}})=\mathbf{B}(\hat{r}, \mathbf{r}) \mathbf{y}(\mathbf{r})=\mathbf{R}_{x x}(\hat{\mathbf{r}}, \mathbf{r})\left[\mathbf{R}_{x x}(\mathbf{r}, \mathbf{r})+\mathbf{R}_{n n}(\mathbf{r}, \mathbf{r})\right]^{-1} \mathbf{y}(\mathbf{r})
$$

The mapping of temperature and salinity was performed directly on anomalies (the time-mean at the mooring locations was removed) rather than full fields, since here our interest is the variability and griding the full fields would overly smooth the background stratification. However, full (mean plus variability) temperature and salinity sections were required to define the water mass interfaces that will be used in calculating transports. These mean sections were obtained combining the mooring data with CTD and direct velocity measurements collected along Line W during the moorings deployment and recovery cruises. The hydrographic sections are fundamental in constructing the mean sections, since they had much finer vertical and horizontal resolution than the moorings and so provided information about the smaller scales. The differences between the time mean profiles from the moorings alone and the gridded mean sections at the moorings locations are on the order of $<0.01^{\circ} \mathrm{C}$ for temperature and $<0.001$ for salinity. The errors in the water mass interface calculation associated with these differences are smaller than the vertical resolution of the objectively mapped data, therefore negligible.

The gridded velocity anomalies, on the other hand, were obtained by first mapping daily estimates of the full velocity field, and then computing and removing the time mean. Unlike temperature and salinity, the observed mean velocity field at Line W 
has little vertical variation (Joyce et al. (2005) and Toole et al. (2010)) and so the mean velocity does not affect the mapping. The resulting velocity anomalies are the same whether the time mean is removed before or after the mapping. However, mapping the full velocity rather than anomalies has the advantage of having the mean and variability sections obtained by the same method, allowing for a more straight forward comparison.

The mapping method described above requires knowledge of the mean and the covariance matrices. This mean, recall, is a single value obtained by averaging all available data for a given variable, and it is different from the time means used in defining the anomalies (functions of $x, z$ ). The covariance matrices we used are Gaussian functions of the distance (in the three dimensional space, $x, z$ and $t$ ) between each pair of points given some correlation lengths $\left(\sigma_{x}, \sigma_{z}\right.$, and $\left.\sigma_{t}\right)$.

$$
\mathbf{R}_{x x}=\langle\mathbf{x}(\mathbf{r}) \mathbf{x}(\mathbf{r})\rangle=P_{0} \exp \left\{-\frac{\left(r_{i}-r_{j}\right)^{2}}{\sigma_{\mathbf{r}}^{2}}\right\}
$$

Where $P_{0}$ is the maximum error variance, and the noise is assumed to be white in the absence of more information.

Our choice of correlation scales was based in two factors: first, the typical scales of the variability, and second, the spacing between observations. Spatial scales for all variables were chosen based on a the Line W hydrographic sections (typical station spacing 10-20km). From these data we found that the horizontal scales of temperature, salinity and velocity anomalies are typically on the order of $100 \mathrm{~km}$. Vertical correlation scales for temperature and salinity anomalies were found to be $500 \mathrm{~m}$ or larger. In the case of the velocity, the fluctuations are coherent through the water column. Based on these scales, the array design, with typical horizontal resolution of $50 \mathrm{~km}$ and vertical spacing between fixed-depth sensors of 100-500m, was not limiting. In order to minimize the misfit between the original data and the objectively mapped product (the longer the scales employed in the mapping, the smoother the final product, and so the larger the misfit) we used a $60 \mathrm{~km}$ horizontal correlation scale and a 
$500 \mathrm{~m}$ vertical correlation scale.

To obtained an estimate of the misfit associated with the selected length scales and array design ${ }^{1}$ we took the hydrographic sections, subsampled them using the mooring sampling scheme and then objectively mapped them back into the original grid. Doing this we found upper bounds for the errors (in the rms sense) of $0.002 \mathrm{~m} / \mathrm{s}$ for the velocity, $0.01 \mathrm{deg} \mathrm{C}$ in temperature and 0.0005 in salinity. The temperature and salinity errors were found to be one order of magnitude smaller when excluding the top $1000 \mathrm{~m}$ of the water column, where the mooring array does a poor job sampling.

The third dimension in the mapping, time, was dealt with in a different way. The decorrelation time scales obtained from the fixed-depth sensors in the moorings (range between 50 and 100days) were strongly controlled by the frequency of rings in the records. Using these decorrelation times would lead to a very smooth field and make for a computationally expensive calculation. In the absence of rings and meanders, correlation scales are much smaller, typically 15-20days. Based on the temporal resolution of the data (5days for the MMP moorings, daily for the fixedsensors) the final product was obtained using a 7day correlation scale. Rms errors caused by the temporal correlation scale were similar to those associated with the spatial mapping.

The analysis presented here only makes use of the well-resolved areas of the mapped fields, given by uncertainties in the mapping smaller than $10 \%$. Regions with larger uncertainties were excluded.

\subsection{Rings, meanders and the mean circulation at Line W}

The upper part of the southernmost mooring at Line W, W5, was frequently embedded in the shoreward core of the mean Gulf Stream, with typical velocities of about

\footnotetext{
${ }^{1}$ Notice that the uncertainty provided by the mapping method is a function of the correlation scale itself; the larger the scales the smaller the uncertainty, so it should not be interpreted as a true error, but as guideline to discard mapped data in the regions that are poorly sampled
} 
$5 \mathrm{~cm} / \mathrm{s}$ (to the northeast). The large horizontal gradients in the Gulf Stream's velocity core imply that even small fluctuations in the position of the Gulf Stream can produce large changes in the velocity observed at W5. These fluctuations are often associated with the baroclinically unstable nature of the Gulf Stream that leads to the formation of rings and meanders (eg. Cronin and Watts (1996)). When rings and meanders are large their effect can also be felt in the shallower moorings at Line W. The density contrast between the Slope Water and the Gulf Stream is also large, so both the density and velocity fluctuations associated with Gulf Stream rings and meanders are responsible for an important part of the variability in the Slope (Csanady and Hamilton (1988)). In fact, the dominant mode of variability in the velocity and temperature record at Line $\mathrm{W}$ is the presence/absence of rings and meanders. In order to investigate the changes in the natural variability of the DWBC, with amplitudes one order of magnitude smaller than the fluctuations caused by the Gulf Stream, we needed to exclude those periods in the record that are affected by rings and meanders.

Gulf Stream rings and meanders exist at a large range of periods and wavelengths (eg. Watts and Johns (1982) and Brown et al. (1986)). They can migrate or be stationary and although they are frequent, filtering the record for a particular frequency band does not ensure their complete elimination, and moreover such a treatment will most likely eliminate other signals of interest within the same frequency band. Instead, our approach to eliminate rings and meanders from the time series was to construct an index based on a idealized representation of the Gulf Stream as a baroclinic jet with a Gaussian velocity core that follows a linear path. The jet is in geostrophic balance, so there is an associated horizontal density gradient, with the warm Sargasso Sea water to the south, and the colder Slope Water to the north. This rectilinear jet represents the Eulerian time mean Gulf Stream. The density and velocity anomalies associated with the passing of rings and meanders are calculated as the difference between the synoptic (perturbed) field and the linear jet.

Gulf Stream meanders are lateral shifts of the entire baroclinic structure of the 
jet (eg. Watts and Johns (1982) and Halkin and Rossby (1985)), and so we modeled them as Gaussian perturbations to the path. Warm Core Rings, on the other hand, are generated when large north-extending meanders detach from the main stream (eg. Joyce et al. (1983) and Brown et al. (1986)) leading to clockwise rotating structures with surface velocities at times as large as $2 \mathrm{~m} / \mathrm{s}$. Thus they may be represented as circular perturbations with the same baroclinic structure as the linear jet, but separated from it (figure 4-2).
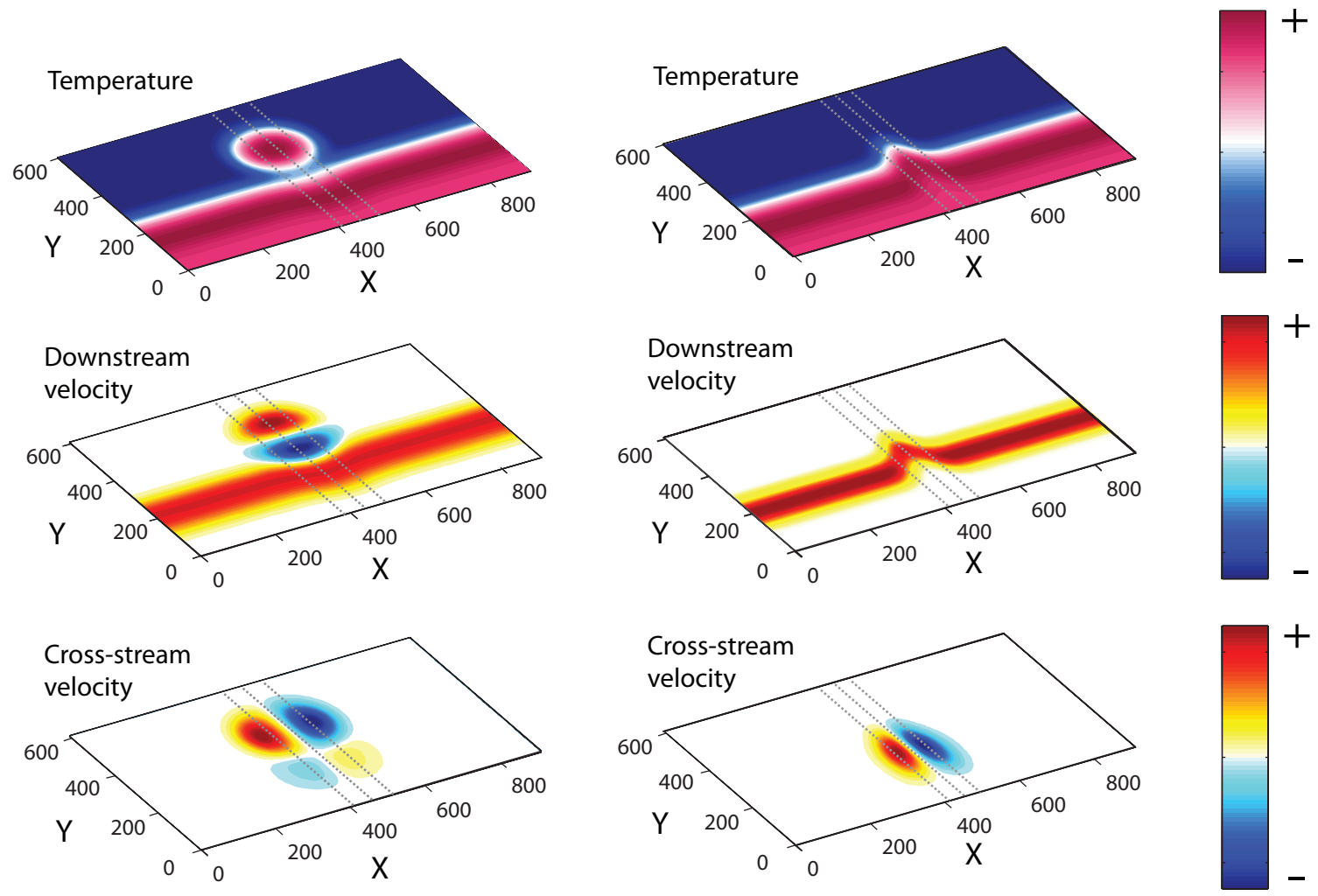

Figure 4-2: Surface views of the temperature, downstream and cross-stream velocity fields for the idealized rings, on the left, and meanders, on the right. The gray dashed lines represent the location of a hypothetical section across the perturbation west of the center, at the center, and east of the center.

Both rings and meanders displace warm Sargasso water to the north, into the much colder Slope Water. As a result, regardless of where in the downstream (alongisobath) direction $(X)$ a section crosses the perturbation, they always have large positive temperature anomalies associated with them. On the other hand, the down- 
stream and cross-stream velocity anomalies associated with a Gulf Stream ring or meander, will depend upon where the hypothetical section crosses the perturbation: upstream (west) from the center or downstream (east) from the center (right at the center the cross-stream velocity is zero). Four possible scenarios characterize a section north of the mean Gulf Stream that is affected by a ring. Defined as combinations of temperature and downstream velocity anomalies, $T^{\prime} u^{\prime}$, and temperature and cross-stream velocity anomalies, $T^{\prime} v^{\prime}$, these are: $\left(T^{\prime} u^{\prime}>0 \mid T^{\prime} u^{\prime}<0, T^{\prime} v^{\prime}>0\right)$, $\left(T^{\prime} u^{\prime}>0 \mid T^{\prime} u^{\prime}<0, T^{\prime} v^{\prime}>0, T^{\prime} v^{\prime}>0\right),\left(T^{\prime} u^{\prime}>0, T^{\prime} v^{\prime}>0\right)$ and $\left(T^{\prime} u^{\prime}>0, T^{\prime} v^{\prime}<0\right)$ (figure 4-3).

The amplitude of the velocity and temperature anomalies caused by rings and meanders is much larger than the variability in the Slope Water. Thus by searching for extreme occurrences of $|T / u \prime|$ and $|T / v \prime|$ combinations in the section we can determine when the array was affected by a ring or meander. Where in the section the extrema occurs is irrelevant. It is the presence or absence of large values that we are after.

Time series of maxima and minima of $T / u \prime$ and $T / v /$ for the objectively mapped Line W data in figure 4-4 shows a very large WCR event in the spring-summer 2006. Both its amplitude (one order of magnitude larger) and duration (8\% of the length of the Line $\mathrm{W}$ time series) are unique in the record. Several other smaller events are seen in the time series as well. We defined ring/meander events as those that exceded $0.1 \mathrm{~m} / \mathrm{s}^{\circ} \mathrm{C}$, equivalent to a $10 \mathrm{~cm} / \mathrm{sec}$ velocity perturbation times a $1^{\circ} \mathrm{C}$ temperature pertubation: values that are typically seen in the Slope Water. Using this value, 15\% of the record was influenced by WCRs and was excluded. Increasing or decreasing the value of the threshold by a factor of 2 had little impact on the percentage of filtered data.

Once rings and meanders were excluded from the record, the resulting mean downstream velocity section (figure 4-5) shows to the north of the weak northeast Gulf Stream flow at W5, three distinct velocity cores: a shallow one centered at W1 at a depth of $1000 \mathrm{~m}$, and two bottom-intensifed cores located at the base of W2 and W4 respectively. Each of these cores have mean velocities of several $\mathrm{cm} / \mathrm{s}$ to the 
RING
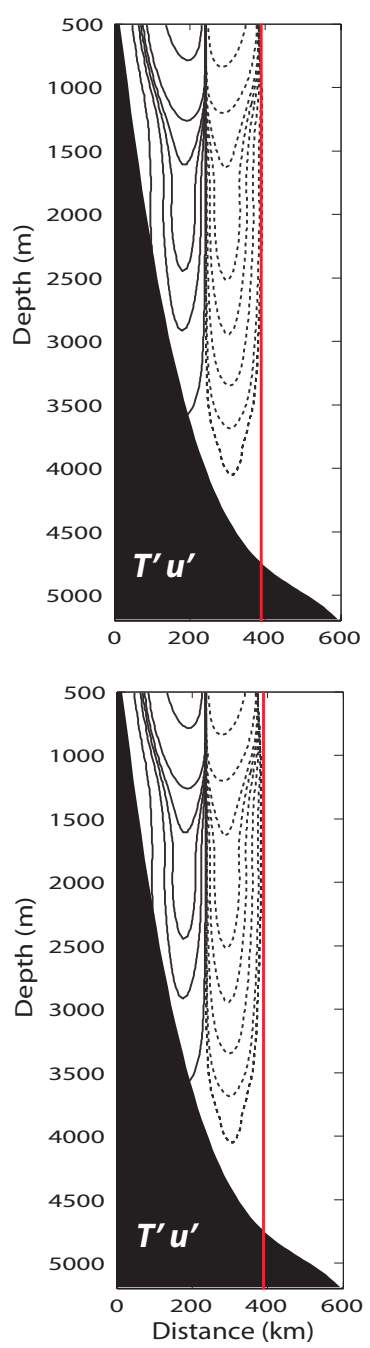
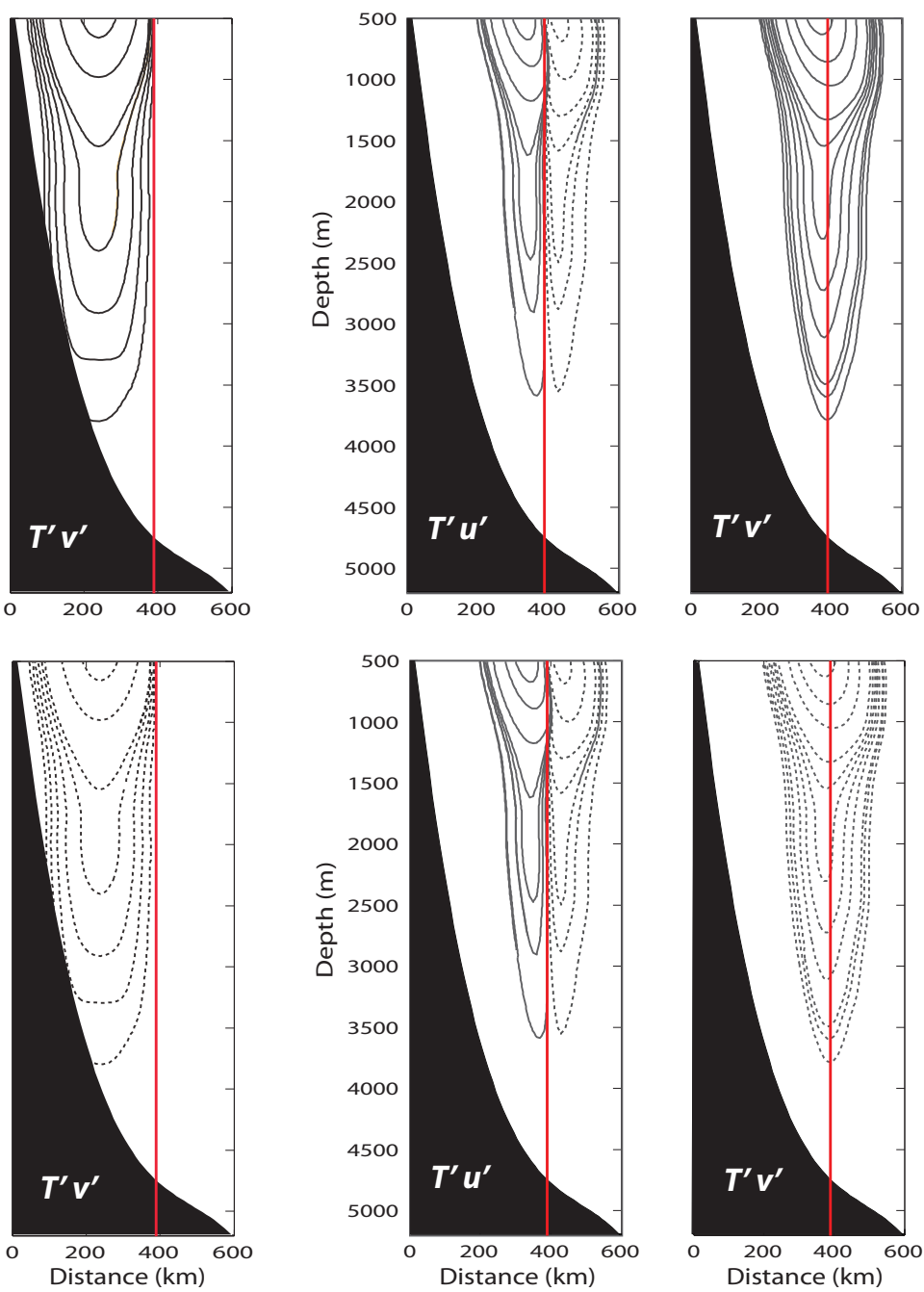

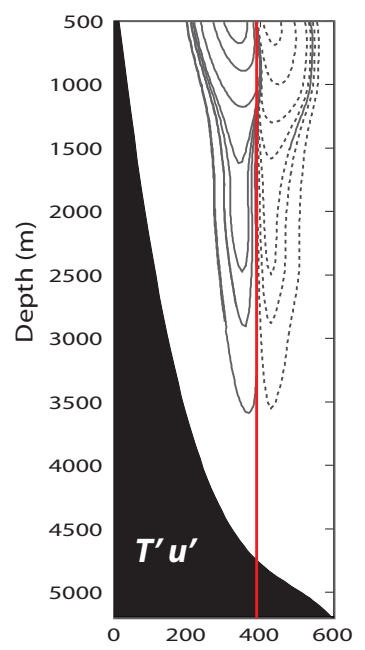

MEANDER

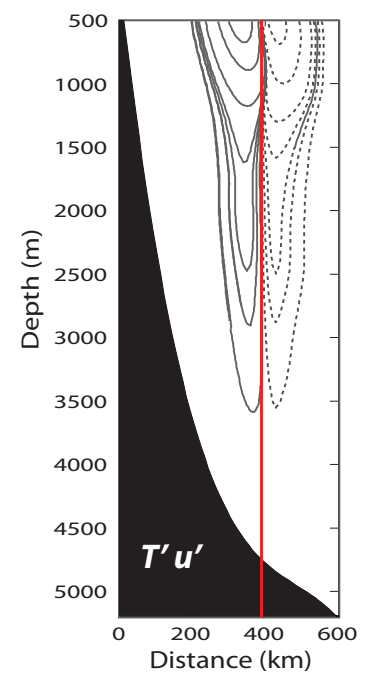

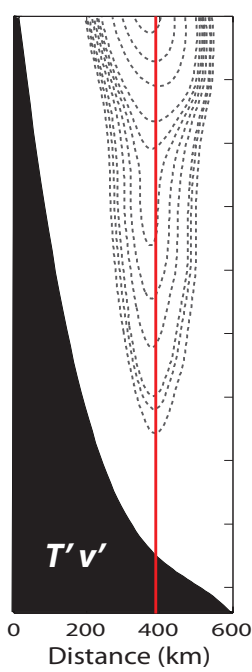

Figure 4-3: Schematic of the combinations of temperature, cross-stream $(v \prime)$ and downstream $(u \prime)$ velocities anomalies associated with rings and meanders. Positive values are shown by the solid lines, and negatives by the dashed lines. A section west of the center of the perturbation is shown on top, and east of the center of the perturbation on the bottom. The red line represents the location of the mean jet.

southwest. Observations of the DWBC both upstream (Schott et al. (2004)) and downstream (Pickart and Smethie (1993)) from Line W also showed bottom intensified flow. However, no evidence of a boundary-intensified velocity core at the level of the uLSW was found by Bower and Hunt (2000) based on float observations, possibly a consequence of the lower vertical resolution of their data compare to ours.

These Eulerian mean velocities, integrated out to the maximum of the southwest- 

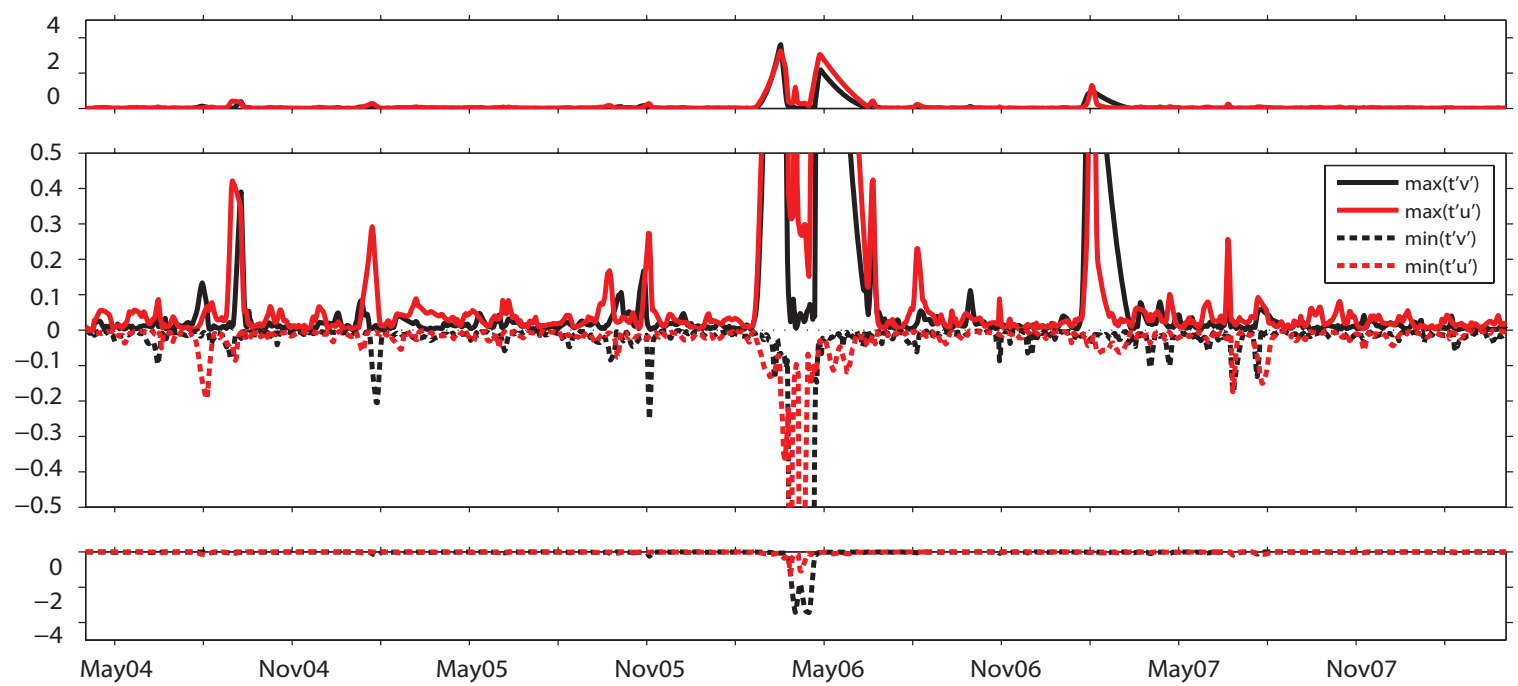

Figure 4-4: The top panel shows time series of $\max \left(T^{\prime} v^{\prime}\right)$ in solid black, $\max \left(T^{\prime} u^{\prime}\right)$ in solid red. The bottom panel shows time series of $\min \left(T^{\prime} v^{\prime}\right)$ in dashed black and $\min \left(T^{\prime} u^{\prime}\right)$ in dashed red. The center panel shows all four time series at the vertical scale of the typical perturbations. The event in the Spring-Summer of 2006 was one order of magnitude larger.

ward streamfunction for the deep and intermediate water masses that are well-resolved by the array, which are dLSW (between the $27.897-27-983 \mathrm{~km} / \mathrm{m}^{3}$ neutral density surfaces), ISOW (27-983-28.066 km/m³) and DSOW $\left(28.066-28.125 \mathrm{~km} / \mathrm{m}^{3}\right)$, translate into mean transport values of -6.8 Sv, $-5.5 \mathrm{~Sv}$ and $-5.5 \mathrm{~Sv}$ for each of the layers respectively. These mean transport are slightly smaller than those obtained by Toole et al. (2010), most likely due to differences in the mapping technique. The uLSW, bounded by the $27.8-27.897 \mathrm{~km} / \mathrm{m}^{3}$ neutral density surfaces, is only partially resolved by the array, so it was excluded.

The mean cross-stream velocity section is slightly divergent. Near the bottom where the flow is mostly steered by bathymetry, the divergence is caused by the spreading of the isobaths upstream of the array. Higher up in the water column the flow transitions from being topographically steered, i.e. roughly perpendicular to the array (the nominal downstream direction), to a more zonal orientation paralleling the mean Gulf Stream axis (a negative cross-stream component coordinate to our rotated axis). 

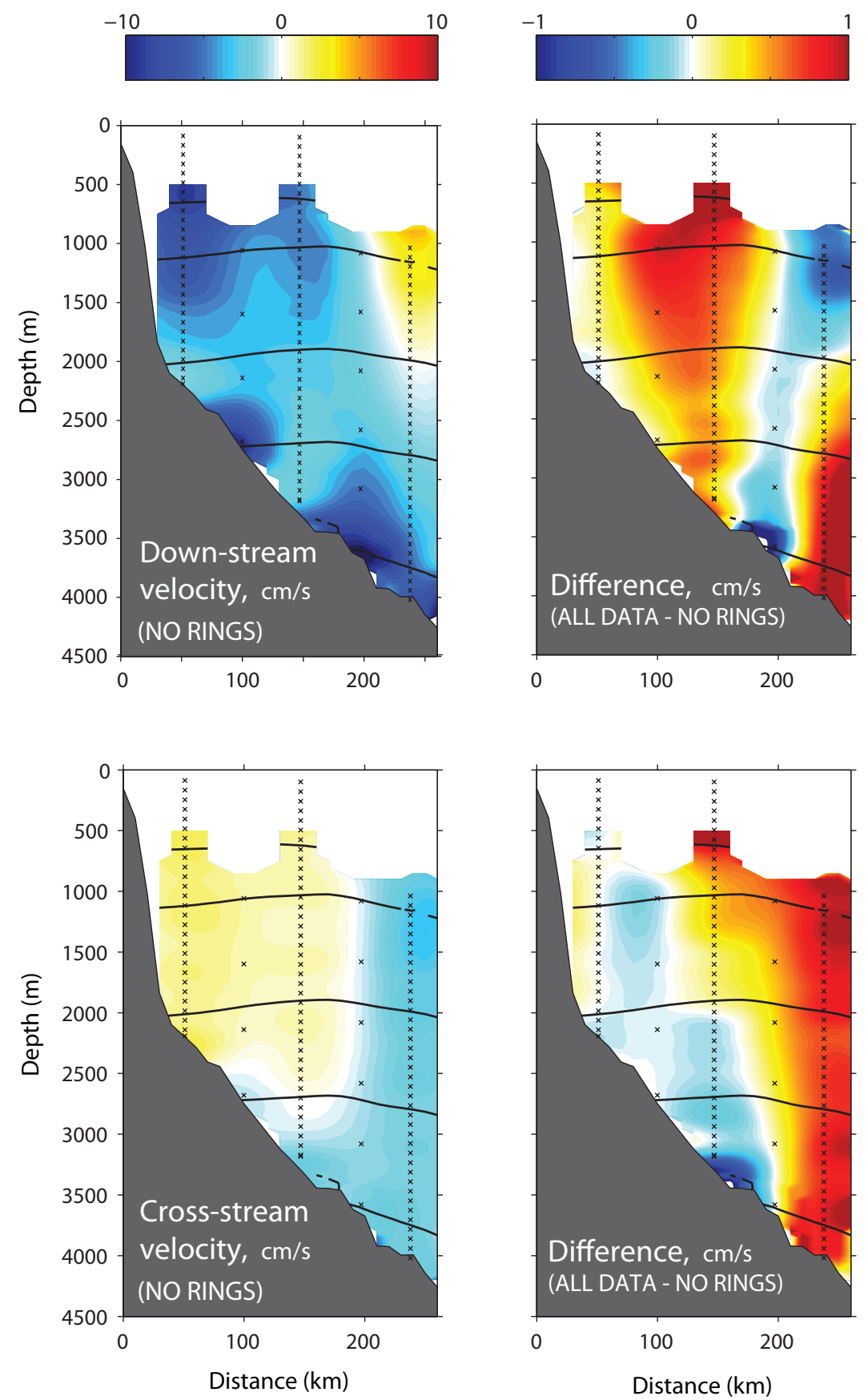

Figure 4-5: On the left downstream and cross-stream mean velocity sections for the ring-meander filter record. On the right is the difference between the mean velocity calculated with the full record, rings and meanders included, and the mean from the ring-meander free record. The solid black lines represent the interfaces between uLSW, dLSW, ISOW and DSOW. Black crosses indicate the location of the moorings. 
Comparing the mean downstream velocity section when all data are included versus that constructed after rings/meanders are excluded (figure 4-5), we find that the rings effectively reduced the southward mean transport across the array by about $10 \%$ (approximately $0.5 \mathrm{~Sv}$ across each layer). The flow that is blocked by the rings/meanders, must be in part deflected to a different path. The deep components of the flow, are most likely deflected offshore, under the deep Gulf Stream. The shallow flow, on the other hand, confined to the north by the Gulf Stream, can only be diverted through an onshore path, or recirculate after colliding with a ring/meander. That was the case in Bower and Hunt (2000) float observations. They found that upon encontouring a warm core ring, the flow in the DWBC at the depth of the upper-LSW, is diverted offshore and recirculates to the northeast on the inside flank of the Gulf Stream.

In the cross-stream component, most of the contribution by rings is located at the offshore end of the section: at the region that is directly affected by the Gulf Stream. The difference implies a reduction in the cross-stream component and it is the result of the somewhat more frequent occurrence of $T^{\prime} v^{\prime}>0$ (a crossing to the west of the center of the ring/meander) than $T^{\prime} v^{\prime}<0$ (a crossing to the east of the center of the ring/meander) events (see figure 4-4).

\subsection{Energetics of the flow}

The distribution of mean flow kinetic energy (MKE) and that of the variability (EKE) show some similarities and some differences (figure 4-6). The deep cores that characterize the mean flow are also present in the EKE, however the upper velocity core, a prominent feature in the MKE, appears to have comparatively little variability. The energy in the variability is concentrated in the deep velocity cores and a band spanning the water column near the Gulf Stream. This rather energetic part of the section near the Gulf Stream has a small contribution to the MKE because it is located right where the meridional flow changes sign. However, is the part of the section where 
even the small fluctuations in the position of the Gulf Stream can have a large impact.

To look at the vertical distribution of fluctuating energy, we calculate frequency spectra of the downstream and cross-stream velocities from the original velocity records at each of the moorings. The velocity records for moorings W2 and W4, equipped with VACMs, as well as the bottom records at W1, W3 and W5, were lowpass filtered (5-day window) in order to eliminate the very high frequencies that are not resolved by the MMPs. The spectra for the upper levels at W1, W3 and W5 were calculated from a number of fixed-depth time series extracted from the MMP profiles. There are some important differences between the measurements from VACMs and MMPs (eg. the different nature of the velocity measurement, sampling schemes, processing, etc.) that will affect the total energy content of the time series (the integral of the spectrum) as well as the distribution of energy among the different frequencies. Hence, the spectra of each type of instrument should be considered separately. The spectra of the downstream velocity, figure 4-7, and cross-stream velocity, in figure 4-8, include all available data, with rings and meanders. The gappy nature of the ring-edited time series prevents us from using classical Fourier analysis.

The downstream component of the velocity fluctuations appears to be bottom intensified for periods shorter than 60 days (figure 4-7). For periods longer than 60 days, surface motions in the downstream direction are more energetic than motions at deeper levels. In general, energy increases offshore, with the shallowest moorings, W1, the least energetic (one order of magnitude smaller), and the deepest, W5, twice as energetic as the middle moorings. Away from the bottom, moorings W3 and W4 show enhanced energy levels around the 100day period. This same period has been previously associated with fluctuations in the transport of the DWBC (Pickart (1994)).

Spectra of the cross-stream component of the velocity (figure 4-8) shows quite a different picture. Overall, cross-stream motions are less energetic than downstream motions by roughly a factor of 2 , with the exception of $\mathrm{W} 1$, which shows very high energy levels around 15 day period. Sites W2 and W4 show motions that tend to be 

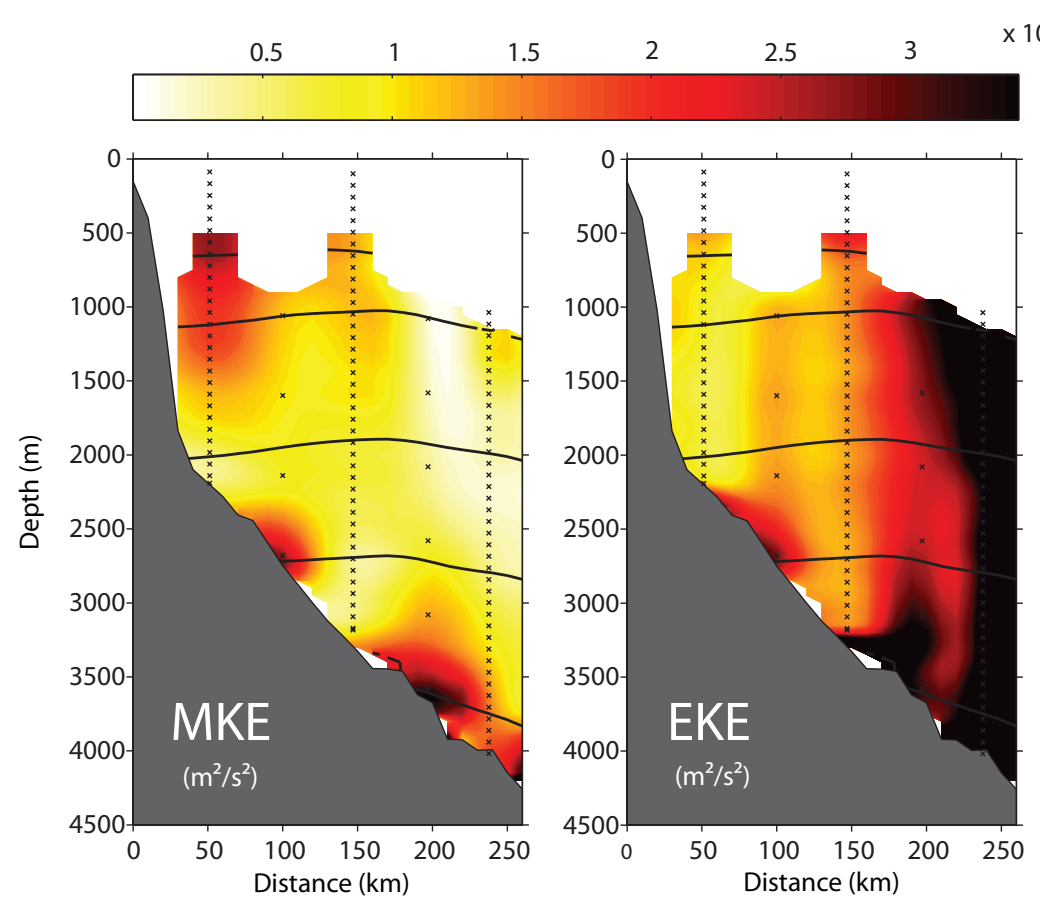

Figure 4-6: Mean and eddy kinetic energy for the the optimally mapped velocity.

more energetic in the upper part of the water column for the entire frequency range, as opposite to what was found in the downstream component.

This asymmetry in the energy distribution of along-isobath and across-isobath motions at different frequencies was already noticed by Thompson (1971) and Thompson and Luyten (1976). Their analysis of moored velocity records in the vicinity of the Gulf Stream highlighted two aspects of the variability of the flow in the area: (1) the flow near the bottom is principally in the along-isobath direction, and (2) the principal axis of the velocity fluctuations depend on frequency. They found that motions at longer periods tend to run back and forth along isobaths, intermediate frequency oscillations fluctuate in the across-isobath direction, and motions at higher frequencies tend to be isotropic. Our calculation does not resolve the high frequency range in Thompson and Luyten's discussions, but the same relation between low and intermediate frequencies appears to be true here. In Thompson's work, the high frequency end of the variability (periods of 1 week) was linked to the passing of topographic Rossby waves (TRW). There are several observations of TRW over the continental 
Downstream velocity
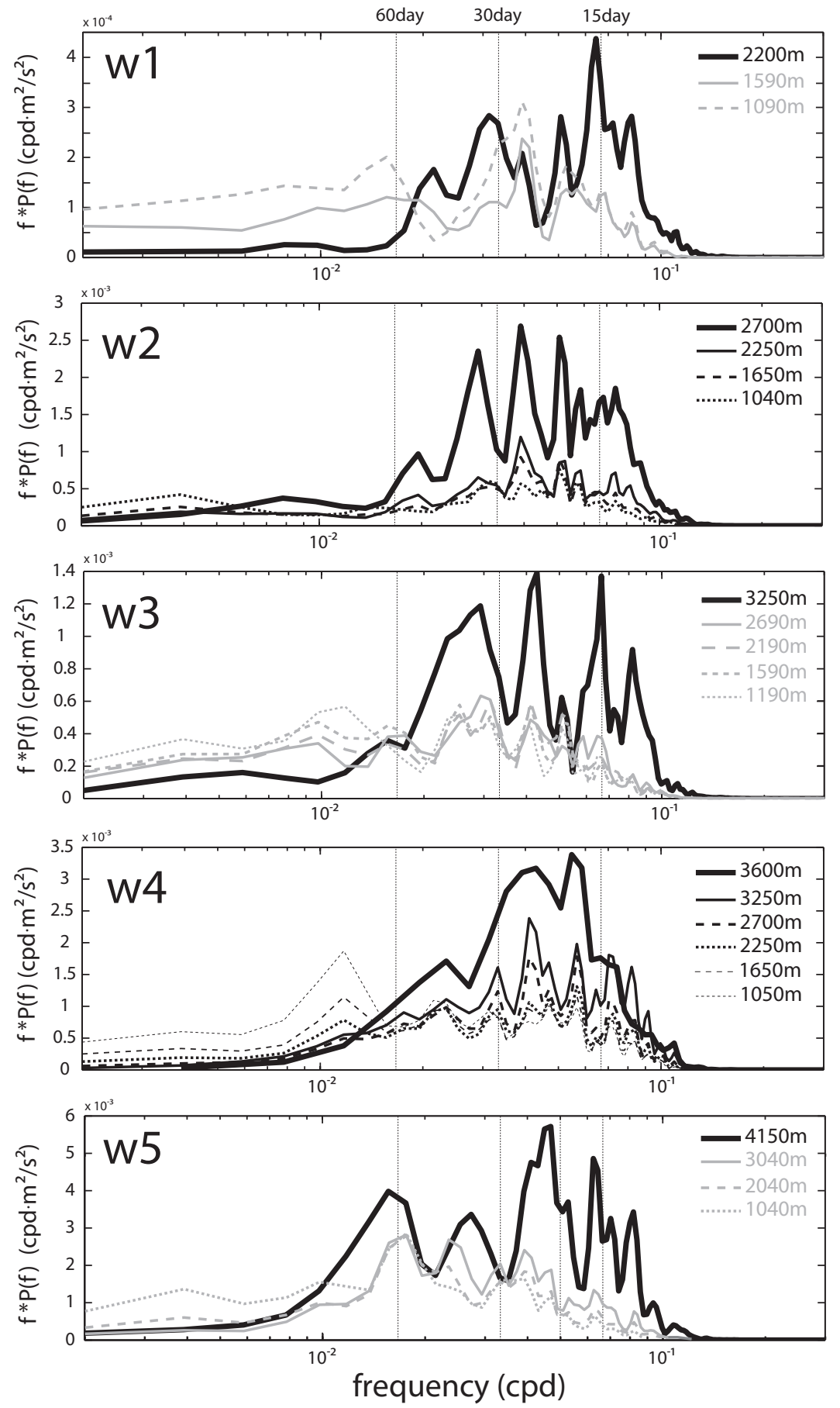

Figure 4-7: Spectra of the downstream component of the velocity. All black lines correspond to depths were VACM records are available -full depth W2 and W4, allmoorings bottom-. Vertical levels extracted out of the MMP records for moorings W1, W3 and W5 are shown in gray. 

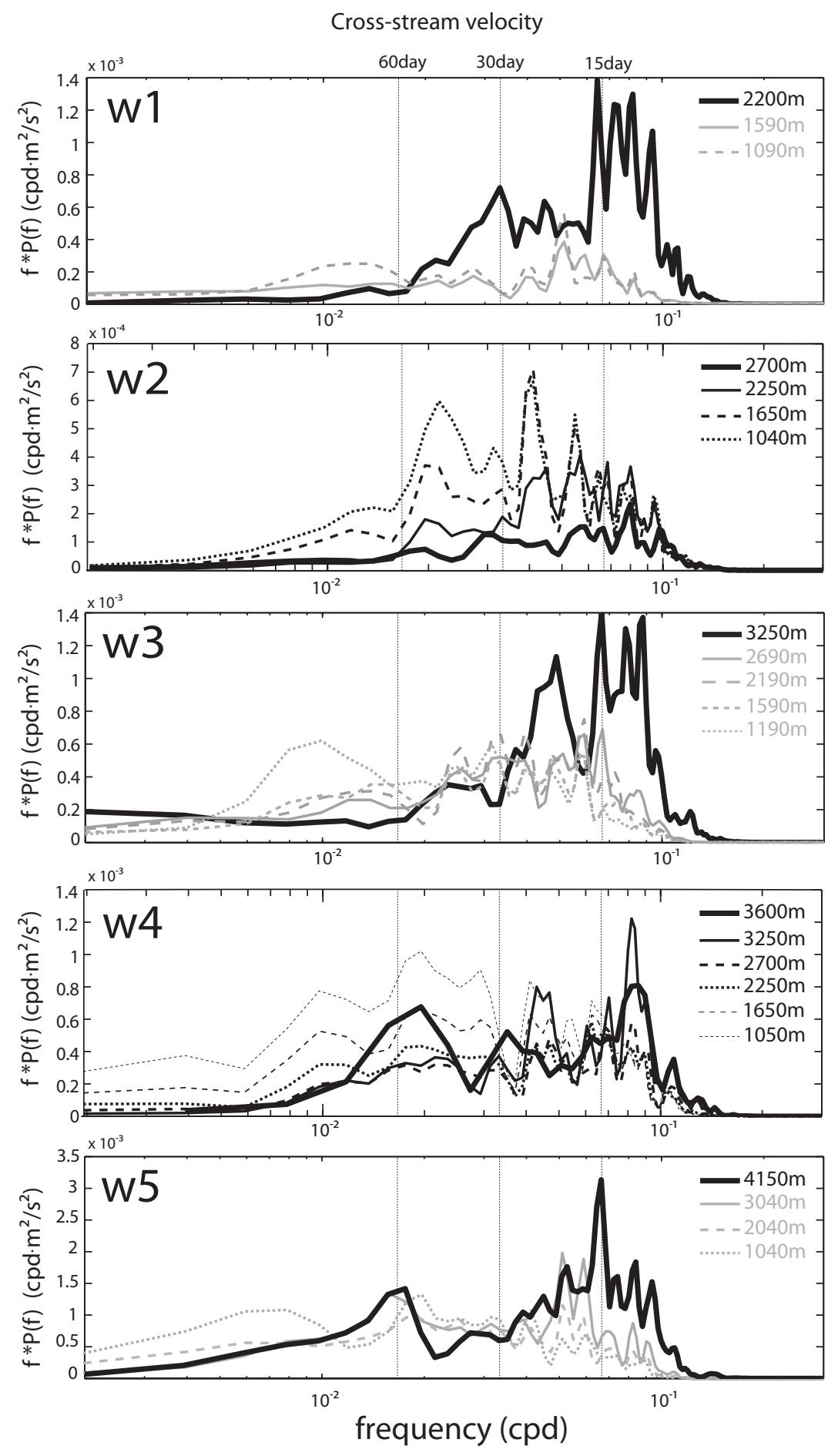

Figure 4-8: Spectra of the cross-stream component of the velocity. All black lines correspond to depths were VACM records are available -full depth W2 and W4, allmoorings bottom-. Vertical levels extracted out of the MMP records for moorings W1, W3 and W5 are shown in gray. 
slope in the western North Atlantic (eg. Pickart and Watts (1990), Pickart (1995) and Frantantoni and Pickart (2003)). The range of frequencies that are often linked in the literature to TRW is somewhat broad. However, considering that according to quasigeostrophic theory, the frequency of TRWs depends on the topographic slope as well as the stratification, and both can be different in different parts of the western North Atlantic, a broad range of frequencies is not unrealistic.

As to what excites TRW, Pickart (1995) argued they can be caused by Gulf Stream instabilities. Both TRWs and Gulf Stream instabilities appear to be important sources of variability in our record. If the occurrence of TRWs is related to the ring/meandering activity of the Gulf Stream, the temporal evolution of the energy in the TRW frequency band, and the Gulf Stream ring/meander index from section 4.3 should show similar behaviors. We explored this relation by means of wavelet analysis ${ }^{1}$. The analysis was restricted to the bottom-most observations, both because motions below $1000 \mathrm{~m}$ appeared to be bottom intensified in the array, and for practical reasons, since all moorings, including profiling moorings, were equipped with VACM at the bottom (so instrumental differences were not an issue).

The kinetic energy wavelet amplitude, in figure 4-9, characterizes the distribution of energy as a function of both frequency and time, whereas regular spectral analysis that only tells us about the energy distribution as a function of frequency. The integral of the wavelet amplitude along the time axis is equivalent to the spectrum. We find that motions in the frequency band corresponding to periods from 15 to 60days, are frequently energetic but have an intermittent nature. This band in the central moorings, W2 and W3, is populated by patches of large amplitude throughout the entire record, but the occurrence of these patches appears to be random.

In order to compare the total energy content within this intermediate frequency band between different moorings, we integrated the wavelet amplitude for the 15day and 60day period band and compared it with ring-meander index from section 4.3. The integral of the wavelet amplitude (figure 4-10) shows that the energy increases

\footnotetext{
${ }^{1}$ We used a complex Morlet wavelet function. For details on this and other aspects of wavelet analysis the reader is referred to Torrence and Compo (1998)
} 

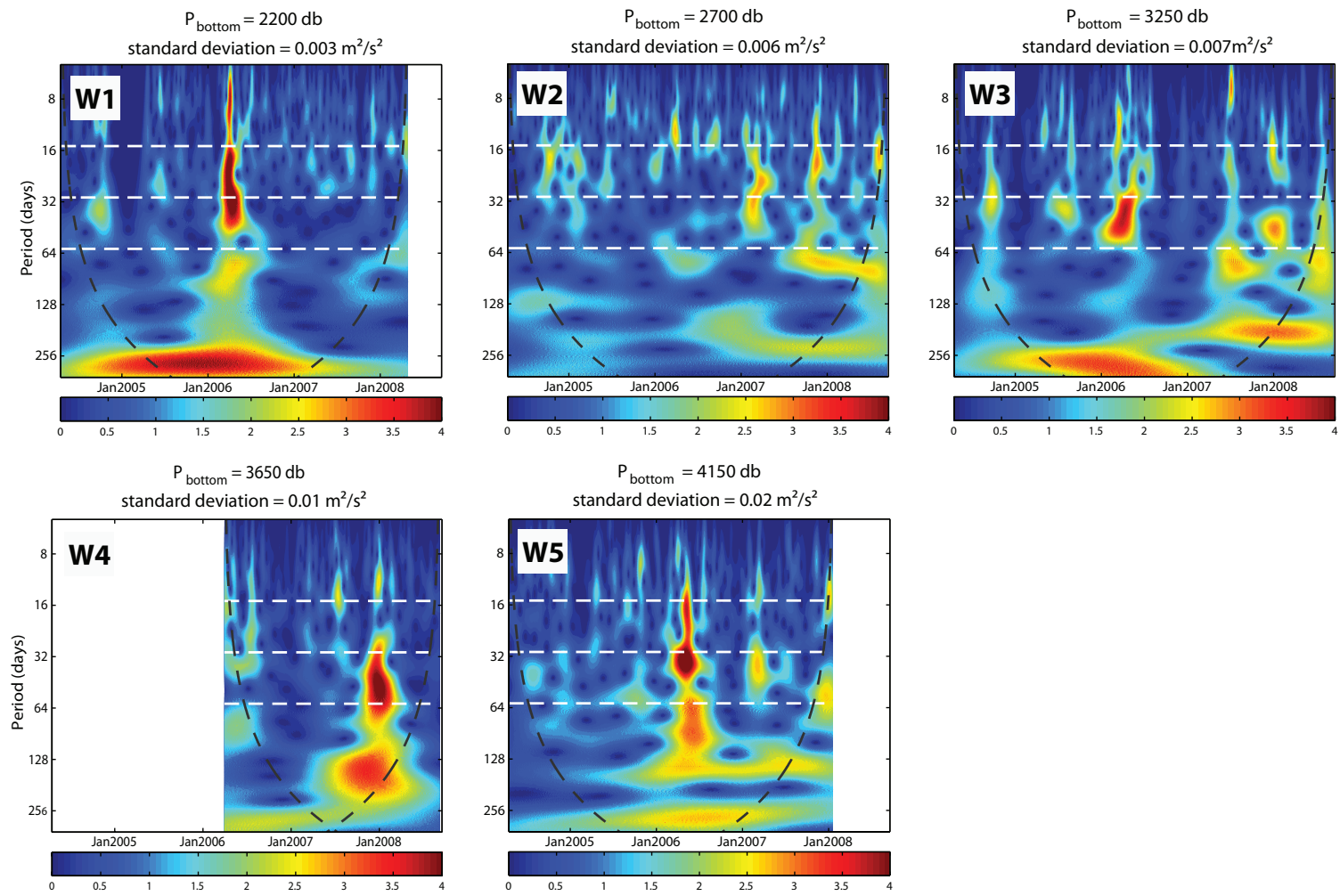

Figure 4-9: Wavelet amplitude of the kinetic energy normalized by the standard deviation for the bottom current meters at all five moorings. Standard deviation values are shown. The white dashed lines are the 15, 30 and 60 day periods. The black dashed lines are the cone of influence where the estimates are not reliable due to edge effects. Due to the particular shape of the Morlet wavelet there is some spreading around the peaks.

offshore, as seen before. The most energetic features in the records appear to be associated with Gulf Stream rings and meanders, consistent with Pickart's (1995) ideas on the generation of TRW by Gulf Stream instabilities, with the exception of a peak during the winter of 2007-2008 observed in W3, W4 and W5. This event will be discussed in some more detail in section 4.7 . 


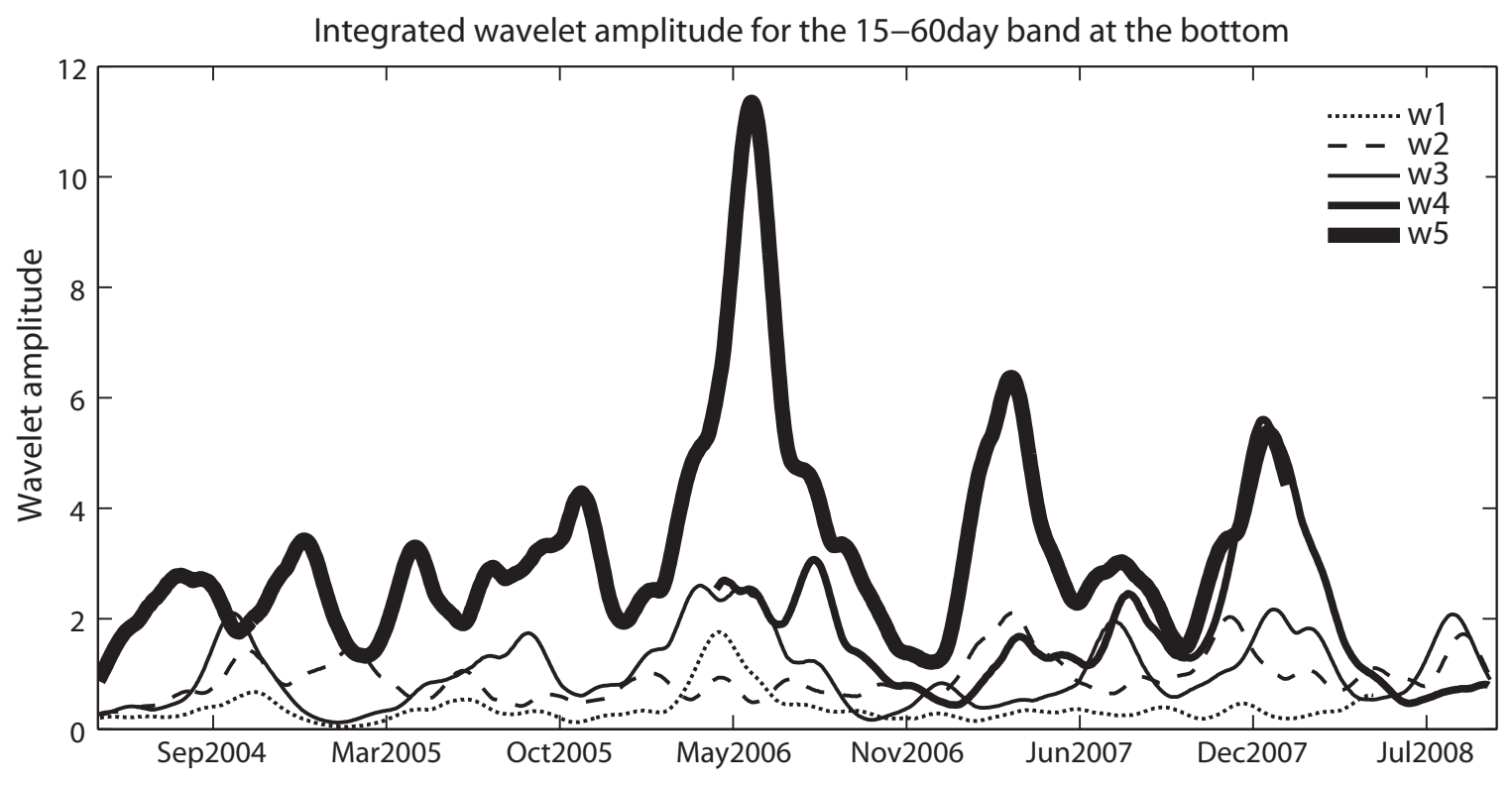

Ring/meander Index

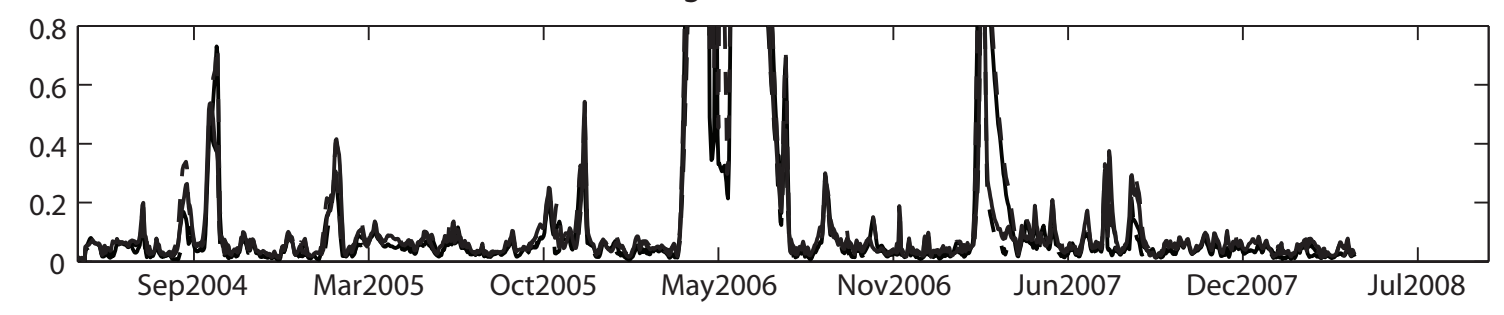

Figure 4-10: The top panel shows the integral of wavelet amplitude for the 15-60 day band for the bottom current meters as a function of time. The bottom panel shows the two ring-meander indexes with positive values.

\subsection{Water mass changes and the response in the circulation.}

Once the contribution from the rings and meanders to the variability of temperature, salinity and velocity fields has been eliminated, the remaining variability, away from the surface where local forcing is still important, must be in part driven by changes in the processes involved in the dense water formation and export. Winter time convection at high latitudes produces water masses that are, in general, cold due to the large heat fluxes, fresh due to the mixing with fresh surface water and weakly stratified 
due to the convective mixing process. This low stratification, and therefore low potential vorticity $\left(\mathrm{PV}\right.$, here defined as $\left.-\left(f / \rho_{0}\right) \partial \rho / \partial z\right)$ may be used as an indicator of the intensity of convection, both inside and outside the convective basin: the more vigorous the convection, the lower the PV signal in the resulting water mass. When convection is weak or it does not occur, the water gradually re-stratifies causing the PV to rise (Talley and McCartney (1982)). Once the water is isolated from the surface, PV tends to be conserved and its signature can be traced as the water spreads away from the basin where it was formed.

To investigate the changes in the circulation of the DWBC that are part of the response to changes in the dense water formation we proceeded in a similar manner as we did with the central mooring, W3, in Chapter 3, and calculated the joint EOF of the velocity, PV, temperature and salinity anomalies (time-mean removed). Here, however, the upper part of the water column (depths smaller than $1000 \mathrm{~m}$ ) where the amplitude of the fluctuations in all fields considered is much larger than at depth, was not included and so the depth-based normalization was not necessary. In addition to the downstream velocity, since the main goal of the full array analysis was to characterize the circulation, the cross-stream component of the flow was also included. The leading mode of the joint EOF analysis of the ring/meander-free time series (figure 4-11) represents $10 \%$ of the variability of the combined fields. The percentage is not high, but the eigenvalues of the analysis are well behaved (North et al. (1982)) and considering that the analysis includes five separated variables with their corresponded noise, $10 \%$ is not insignificant. Part of the signal associated with rings and meanders is still present in the record. It appears to be distributed in higher modes with a nearly barotropic velocity pattern that resembles the structure of the idealized rings and meanders shown in figure 4-3, that fluctuate at periods of several days to a few weeks.

The PV spatial mode shows a distinct layering pattern, with a positive PV anomaly at intermediate depths occupying the depth range of the dLSW and upper part of the ISOW, and negative PV anomalies above and below, in the uLSW 

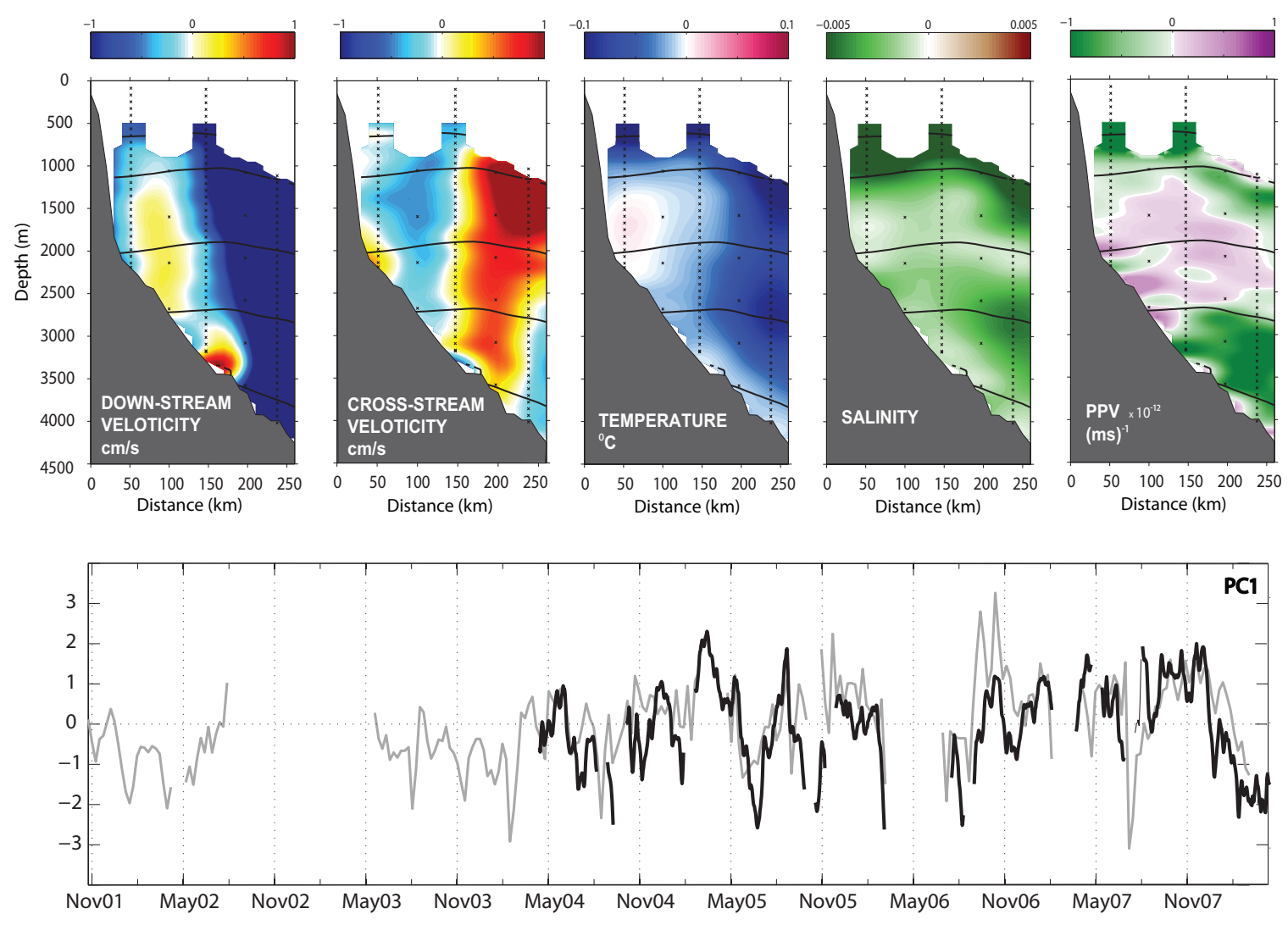

Figure 4-11: Leading mode of the joint EOF of the downstream velocity, cross-stream velocity, potential temperature, salinity and PPV -from left to right-. The black contours represent the interfaces between upper-LSW, deep-LSW, ISOW and DSOW, from top to bottom. The bottom panel shows in black the corresponding principal component, and in gray the leading principal component of the joint EOF analysis from the W3 record alone presented in Chapter 3, figure 3.N.

and DSOW layers. These anomalies are laterally coherent throughout the section. During the phase when the PV anomaly is positive at the level of the dLSW (when $\mathrm{PC} 1>0)$ the section is fresher and colder every where except for a small region at mid-depth in the vicinity of W1 where some weak warming is indicated. This warm core is only partially compensated by salinity, since there is no actual increase in the salinity at that location, only a weaker freshening, and is probably related to changes in the LSW core properties. Taking the PV signal as an indicator of ventilation for the LSW, our section is consistent with Dickson et al. (1996) finding of coordinated changes in convection in the Labrador and Nordic Seas. They found 
that during phases of deep convection in the Labrador Sea (corresponding to $\mathrm{PC} 1<0$ phases in our analysis) only shallow convection occurs in the Nordic Seas, leading to progressively warmer and more saline OW due to increased exchange with the Arctic. However, this warming tendency is not exclusive to the deep layers at Line W but affects most of the water column, with the exception of the temperature core at W1 mentioned earlier. We will show in the next chapter that this depth-independent part of the salinity and temperature changes is caused by the rising of the isopycnals associated with the expansion of the OW layer (negative PV).

The velocity signal that accompanies these changes in PV, temperature and salinity described above, consists of a large negative anomaly (values ranging from -5 to $-10 \mathrm{~cm} / \mathrm{s}$ ) in the deep part of the section, a weaker positive anomaly (maximum values of $1 \mathrm{~cm} / \mathrm{s}$ ) in the shallow region below $1000 \mathrm{~m}$ centered at W2, and a larger positive core near the bottom between W3 and W4. The positive anomaly at W2 is isolated from the uLSW layer on top, where changes in the velocity are in-phase with changes in the offshore part of the section. The amplitude of the positive anomalies is small compared to the mean downstream flow in this part of the array. During positive phases $(\mathrm{PC} 1>0)$, these anomalies act to decrease the southwestward mean flow, and during negative phases, they reinforce it. The effect of these anomalies in the transport integrated across the section will be discussed in the next section.

Changes in the velocity are not restricted to the downstream component; the cross-stream velocity mode indicates that there are important directional changes as well. The mode represents a confluent pattern, with negative values (offshore) at shallow depths and positive values (inshore) in the deep part at times when PC1>0. The opposite is true when the mode reverses.

PC1 shows frequent reversals throughout the record (bottom panel figure 4-11), but we find that on average PC1 was mostly negative pre-2006, thus negative PV at intermediate depths, and positive after, with negative PV in the upper and deeper layers. This tendency is further supported by comparing PC1 to the principal component the joint EOF of velocity, temperature, salinity and PV for the record at W3 in 
Chapter 3, in which the overall trend is much more clear (bottom panel figure 4-11).

\subsection{Intermediate and deep water transport}

In the previous section we have shown that changes in the water mass structure at Line $\mathrm{W}$ are accompanied by changes in the amplitude, direction and spatial structure of the flow. In this section we explore the effect of these changes in the equatorward transport of the DWBC. We do so by considering the fluctuations in the downstream component of the velocity only, since this is the direction of the mean flow in the DWBC and the cross-stream component does not contribute to the transport normal to the array.

The transport response to changes in formation and in export of deep and intermediate water masses along the DWBC was calculated by combining the joint EOF downstream velocity mode and mode-derived density variability with the velocity and density mean sections. The mean density section is only required to calculate the interfaces between the different water masses, and so the errors due to the difference in the mapping of $\mathrm{T} / \mathrm{S}$ means and $\mathrm{T} / \mathrm{S}$ variability are negligible, as discussed in section 4.2.2. The downstream velocity, given by the sum of the EOF1 velocity mode and the mean velocity section, is then integrated across each layer out to the maximum in the transport streamfunction (only the equatoward flow). The position of the streamfunction maximum in this calculation depends only on the location of the Gulf Stream's North Wall, due to the fact that the velocity anomalies from the EOF mode are never large enough to reverse the southwestward mean flow.

The changes in the density field and the circulation captured by the joint EOF analysis show a relatively barotropic response in the transport (top panel figure 412). DLSW, ISOW and DSOW transports appear to fluctuate in phase. There are three possible variables that contribute to the fluctuations in the transport: (1) the averaged layer velocity, (2) the offshore extension of the layer (given by the location of the streamfunction maximum) and (3) the averaged layer thickness. Changes in 

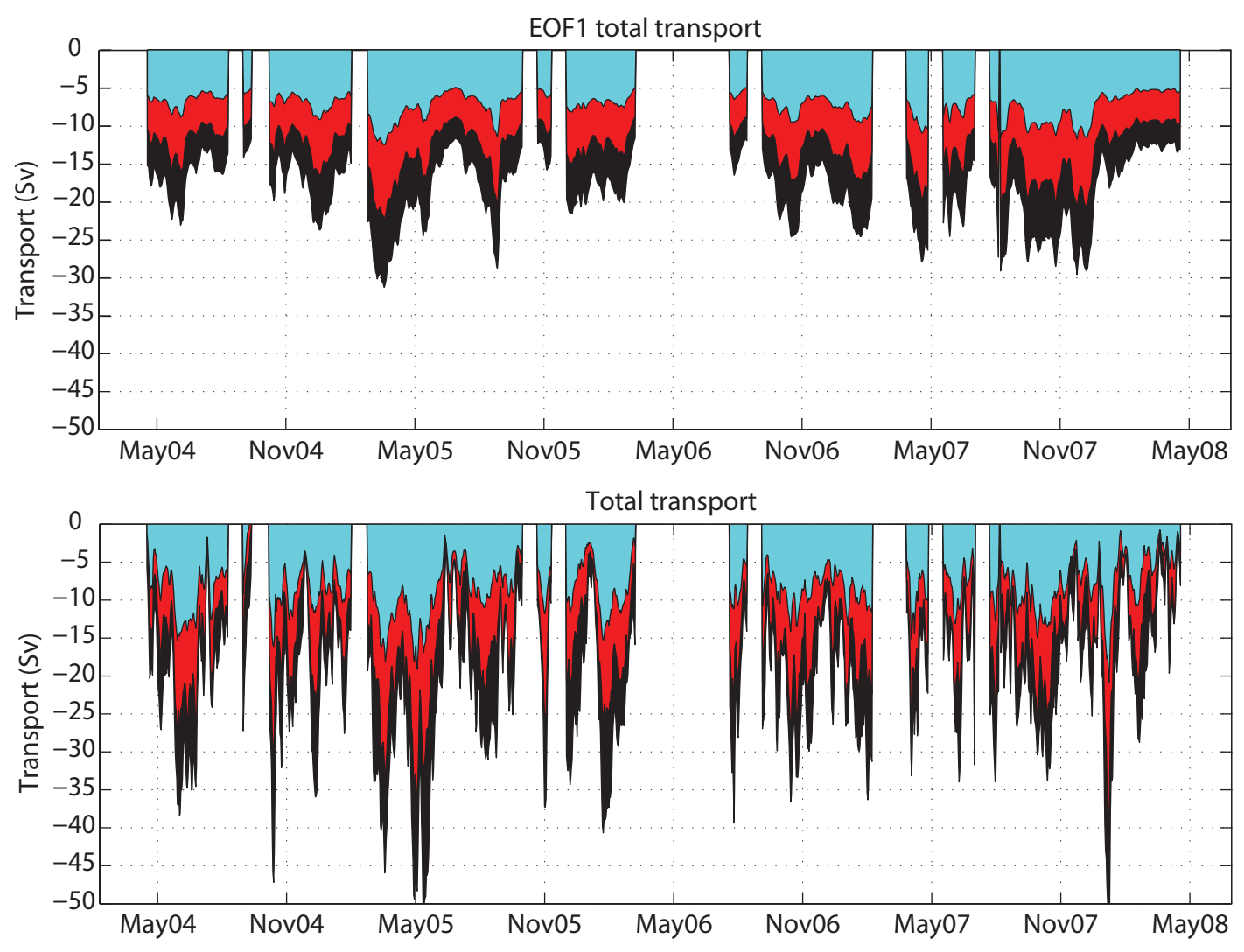

Figure 4-12: Transport, in Sverdrups, integrated up to the maximum SW transport streamfunction for the dLSW, in blue, ISOW, in red, and DSOW in black. The top panel shows the transport integrated from the combined EOF mode's density and downstream velocity fields. the bottom panel shows the transport obtained using the full velocity field.

(1) and (2) are in phase for all three water masses, and are responsible for the bulk of the changes in the transport, hence its barotropic nature. Layer thickness changes, on the other hand, are different for each layer. However, their contribution to the total transport is small. Overall, EOF1 is responsible for changes of ca. $5 \mathrm{~Sv}$ in each layer. There is evidence of interannual variability in the time series, but given the length of the record we can not conclude there is a significant trend. The maximum transports occur during the Spring in 2005 and Fall in 2007. Towards the end of the record there is a rapid decrease in the transport of about $15 \mathrm{~Sv}$. Although there are other relatively large changes happening over short periods of time in the record, this Fall 2007 event is particularly fast, and the low transport values are sustained until 
the end of the record. The details of this event will be addressed in the next section.

The relation between changes in the PV of the different water masses and changes in the transport is such that the transport over the full extent of the section increases when PV is high in the dLSW layer, and negative at the OW depth range. As we showed in Chapter 3, the evolution of upper and deep LSW also have opposite phase, since only one of the two types is formed in the Labrador Sea in a given winter, and so the export of recently ventilated uLSW is also associated with increased southwestward transport, as we already anticipated from the single mooring analysis. In this scenario, our limited observational record suggests that enhanced convection in the Labrador Sea, the type that results in the production of dLSW, does not lead to a strengthening of the DWBC, therefore the MOC, in agreement with Pickart and Spall (2007) results. It is the presence of recently ventilated OW that is associated with a stronger DWBC, similar to what is found in Gerdes and Koberle (1995) modeling work, among others.

We note that this transport mode was obtained from a mode of variability that represents only $10 \%$ of the variability of the individual fields. How well does it compare with the total transports across the array? If we calculate the transports for the same layers using the full velocity and density fields from the ring-meander free objectively mapped data (bottom panel figure 4-12), we find that the fluctuations in the total transport have shorter period than those captured by the mode, and the amplitudes are nearly twice as large. Nevertheless, the mode does a fair job in reproducing the low frequency variability in the full record. Total transports, like the EOF1-derived transports, have no significant trend. However, there are hints of a trend in the ratio between intermediate and deep water masses, mostly reflecting the contribution from the changes in the layer thickness, which are much smaller than the other factors controling the transport (average velocity and lateral extent). 


\subsection{The winter 2007-2008 rapid event}

PC1 from the joint EOF analysis and the transport time series derived from it show a sudden change in the conditions during the winter of 2007-2008. In transport, this change is characterized by drop of approximately 15Sv distributed over the dLSW, ISOW and DSOW layers. In PV, this abrupt change is coincident with a redistribution from positive PV anomalies in the intermediate layers, and negative above and below, before the winter 2007-2008, to negative PV anomalies at intermediate depths, and positive above and below after. How exactly these changes affect the structure of the circulation at Line $\mathrm{W}$ can be better understood by looking at the sum of the EOF1 velocity mode and the mean velocity section before and after the winter of 2007-2008 (figure 4-13).

There are two major changes taking place during this transition that can explain the sudden decrease in the transport. One is related to the north-south displacement of the Gulf Stream and the other one has to do with the reorganization of the flow around the deep and shallow velocity cores. Prior to the winter 2007-2008, the Gulf Stream's North Wall, found in the mean between W4 and W5, was south of W5, beyond the array limits. The flow at the top of mooring W5 was to the southwest at this time. The largest velocities were found in the deepest of the velocity cores, slightly displaced offshore from its usual location at the bottom of W4 to the bottom of W5. The shallowest of the deep cores typically located around $2500 \mathrm{~m}$, we will call it the intermediate core to distinguish it from the one just discussed, was weaker and somewhat isolated from the surrounding flow. The shallow velocity core was also weak. After the transition, the positive flow in the deep Gulf Stream penetrated all the way to the bottom at W5. The zero-velocity contour was north of W4. In the upper part of the array, both the shallow and intermediate velocity cores intensified, and the flow became relatively barotropic everywhere north of W3. The deep core at the base of W4 was weaker and isolated from the rest of the southward flow. This separation of the deep velocity core is also evident in the cross-stream velocity (bottom 

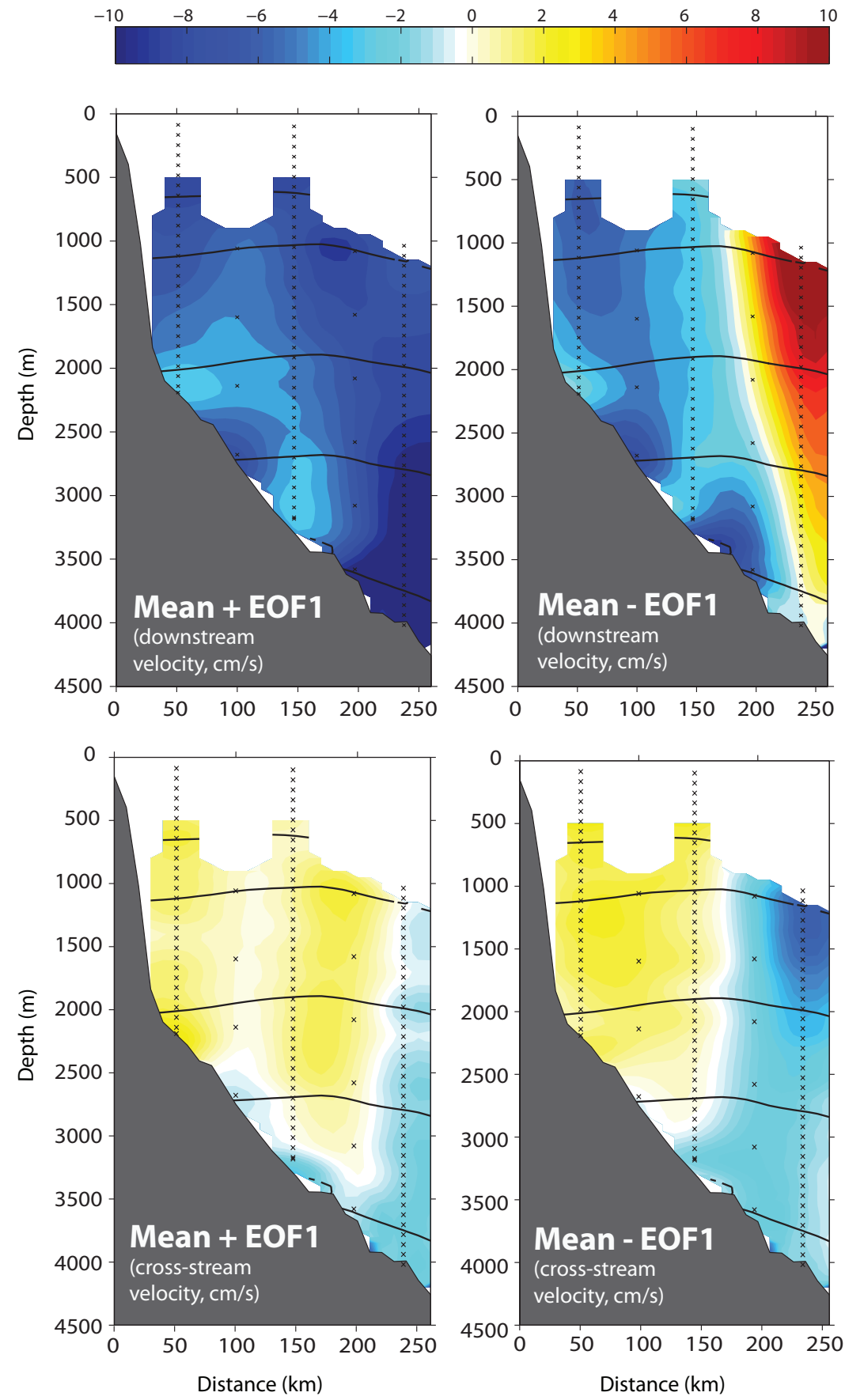

Figure 4-13: Total downstream and cross-stream velocity before the winter 2007-2008 on the left, and after (mean - EOF1) on the right. These conditions are typical of all periods when PC1 is large and positive (mean + EOF1) or large and negative (mean + EOF1). 


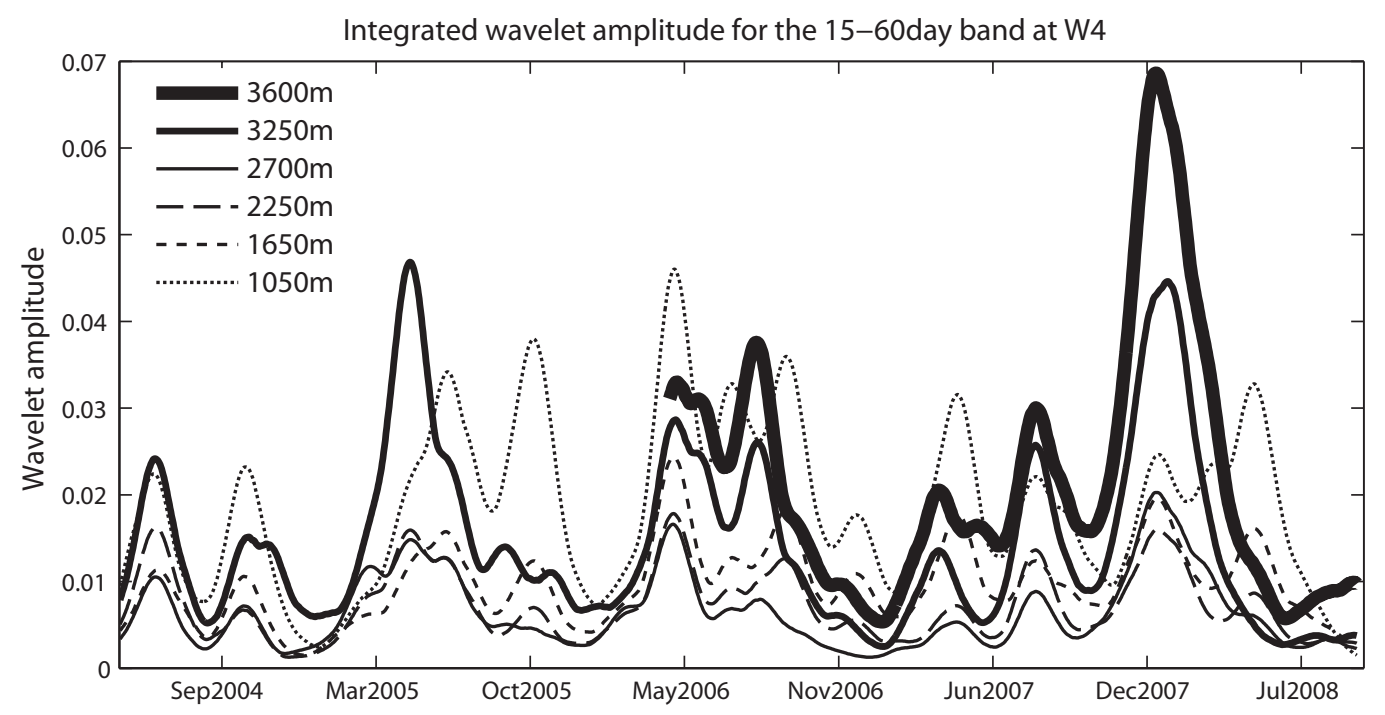

Figure 4-14: Integral of the wavelet amplitude of the kinetic energy from mooring W4 at different depths. No data for the bottom current meter was available for the first two years of the record.

panels figure 4-13). While the flow of intermediate and shallow water remained mostly perpendicular to the array (approximately in the along-isobath direction), the deep velocity core appeared to be steered by the flow in the deep Gulf Stream, which is more zonal and has a negative cross-stream velocity component in our rotated reference frame.

According to the description of the changes in the Gulf Stream path in Chapter 2 , there are two types of large scale shifts: lateral displacement of the current axis, and changes in the meridional tilt. The lateral displacements, which are the leading mode over the whole Slope Water, have smaller amplitude in the shallow moorings at Line $\mathrm{W}$ (W1, 2 and 3), where the meridional tilt mode dominates. In these moorings, the rapid change of 2007-2008 in Line W is better captured by the principal component associated with the meridional tilt mode (figure 2-2). However, at the offshore moorings (W4 and W5), the contributions from lateral displacements and changes in the meridional tilt of the Gulf Stream are equally important.

As a result of the changes in the position of the Gulf Stream and the associated reorganization of the deep flow, W4 experienced some of the largest fluctuations in 
the velocity of the entire array during the whole record. The magnitude of these fluctuations can be seen in the integrated wavelet amplitude of the kinetic energy calculated from the original current meter data at different depths (figure 4-14). The wavelet amplitude shows that motions are most energetic near the bottom, around the deep velocity core, and at the top of the mooring (ca. 1000m) right at the base of the surface intensified flow in the Gulf Stream. The peak in wavelet amplitude for the winter of 2007-2008 is the most energetic event in the record at W4 and in the whole array only exceeded by the large ring event in 2006 (see figure 4-10). As discussed in section 4.4, this is the only energetic event in the TRW frequency band that is unrelated to Gulf Stream rings and meanders. Significantly, TRWs are not only forced by Gulf Stream instabilities. The work by Hallberg and Rhines (1996) showed that the response to thermohaline forcing in a basin with sloping boundaries occured mostly via topographic Rossby waves. Along the sloping boundary, the topographic $\beta$-effect is much larger than the planetary $\beta$, so the waves propagate along the boundary leaving the shallow water to the right, downstream in the DWBC sense. Gascard and Clarke (1983) observed TRWs in the Labrador Current within the Labrador Sea during the winter. They hypothesized these waves were the response of the horizontal velocity field to deep convection, which occured in the Labrador Sea in the winter of 2007-2008 (Våge et al. (2009) and Yashayaev and Loder (2009)). So it is possible that these energetic motions that we see at W4 are the rapid response to deep convection in the Labrador Sea, and as they propagate they cause changes in the circulation of the western North Atlantic that affect both the Gulf Stream and the DWBC. According to Roussenov et al. (2008) the topographic wave response to changes in the Labrador Sea could be felt in the western North Atlantic as fast as 1 month after the forcing began. 


\subsection{Summary and discussion}

The mean DWBC inferred from the Line W moored array observations for the period 2004-2008 consists of three distinct velocity cores, two bottom ones located at the base of the dLSW ( $2500 \mathrm{~m}$ isobath) and the DSOW (3500m isobath), and a shallow one within the depth range of the uLSW approximately along the $2000 \mathrm{~m}$ isobath. These cores have typical velocities of $5-10 \mathrm{~cm} / \mathrm{s}$ to the southwest, and are embedded in a background flow that is also directed to the southwest but with velocities that are weaker by a factor of two. The southwestward flow in the DWBC is bounded offshore by the deep Gulf Stream, with velocities of a few cm/s near the North Wall in the upper $2000 \mathrm{~m}$ of the water column.

The time series of velocities measured by the array showed energetic motions at a wide range of frequencies, with a significant amount of energy concentrated near the bottom and at the offshore end of the array. Velocity fluctuations near the bottom occurred in the along-isobath direction mostly with periods ranging from two weeks to two months. Similar along-isobath bottom intensified motions were observed by Thompson (1971) and Thompson and Luyten (1976) in the region, who linked them to the presence of topographic Rossby waves. Topographic Rossby waves are a well-known source of variability in this area (eg. Pickart and Watts (1990) and Frantantoni and Pickart (2003)) and can be generated by Gulf Stream instabilities (Pickart (1995)), which are frequent in the Line $\mathrm{W}$ record in the form of meanders

and leading to the formation of rings. Rings and meanders, when present in the array, can cause fluctuations in the velocity and density fields that are one order of magnitude larger than the climate-related variability of the Slope Water and DWBC. Approximately $15 \%$ of the 4 -year record at Line $\mathrm{W}$ is affected by them. The integrated effect of both rings and meanders in the 4-year record mean is a reduction of the southwestard flow across the array by a few Sverdrups. This is consistent with Bower and Hunt (2000) observations. They showed that the trajectories of floats deployed in the DWBC at the uLSW depth range could be deflected into the Gulf Stream when 
colliding with warm core rings.

When rings and meanders were excluded from the record, the mode of variability in the water mass composition that emerged from the joint EOF analysis of the velocity, temperature, salinity and PV consisted of positive PV anomalies at intermediate depths confined between anomalies of the opposite sign in the water above and below. Using the smallness of the PV values as an indicator of recent ventilation (Talley and McCartney (1982)) we found that on average prior to 2006, the recently ventilated dLSW had a stronger presence at Line W (negative PV anomalies at intermediate depths). During this time, the uLSW and OW depth ranges were characterized by higher stratification values, and waters that were warmer and more saline than the mean, all indicative of lack of the ventilation. This out-of-phase behavior of the deep and upper LSW, the latter only partially resolved by the array, can be caused by changes in the forcing over the Labrador Sea. During harsh winters, typically positive NAO years, deep convection occurs and it leads to the formation of dLSW. When conditions are less severe, convection affects shallower layers and only uLSW is formed (eg. Schott et al. (2004) and Yashayaev (2007)). The out-of-phase relation between the dLSW and the OW, on the other hand, is the result of the same phase of the atmospheric forcing acting on different basins (Dickson et al. (1996)). During negative NAO years, air temperatures over the Greenland Sea are low, leading to larger heat fluxes (from the ocean to the atmosphere) and more ventilation of deep waters. In the Labrador Sea, low NAO years are associated with weaker than normal westerlies and reduced buoyancy forcing, inhibiting deep convection. Alternatively, this out-of-phase behavior between OW and LSW could also be the result of changes in the the Labrador Sea alone, and the deepening of the interface between the dLSW and the OW being just a consequence of deep convection (Sarafanov et al. (2009)).

In coordination with changes in the water mass distribution, we observed changes in the circulation that affect both the DWBC and the Gulf Stream. During phases when recently ventilated dLSW is present at the array, the deep flow (to the southwest) between the 1500 and the $3500 \mathrm{~m}$ isobaths increased, but the transport inte- 
grated across the DWBC decreased, opposite to the traditional view of enhanced LSW production leading to the strengthening of the MOC. This decrease in the transport was due to both a decrease in the lateral extent of the DWBC as well as a decrease in the southward flow in the deep water. Larger DWBC transports at Line W are associated with colder, fresher and weaker-stratified uLSW and OW, as often seen in modeling experiments (eg. Gerdes and Koberle (1995), Böning et al. (1996) and Kohl and Stammer (2008)).

The expansion/contraction of the DWBC we observed was associated with northsouth displacements of the Gulf Stream. These displacements at Line W are mostly due to changes in the meridional tilt of the Gulf Stream in the inner part of the array, and a combination of lateral shifts and changes in the meridional tilt at the offshore-most moorings. When the Gulf Stream is south of the mean at the moorings site, its path away from the separation point tends to be more zonal, and a northerly Gulf Stream corresponding to a more meridional path. Whether these changes in the Gulf Stream path are synchronized with changes in the water mass composition and velocity in the DWBC as a result of coordinated changes in their individual forcing, or if there is a cause-effect relation between the two, is unclear from the data. However, some supporting evidence for the former is given in Häkkinen and Rhines (2009) analysis of the wind stress over the Slope Water. They showed that for the period of 2001-2005 the region between Cape Hatteras and the Nordic Sills was characterized by a positive curl which resulted in a more tilted zero-curl line. These changes in the wind stress curl could potentially drive changes in the path of the Gulf Stream as shown by Kelly et al. (1996).

The analysis of two additional years of data from the central mooring at Line W, W3, acquired prior to the full array deployment, suggested that the changes described here are part of a longer-term change in the DWBC water mass composition from a dLSW-dominant period in the early 2000s to a uLSW and OW period in the later part of the decade. This gradual trend was suddenly interrupted during the winter of 2007-2008, coinciding with the return of deep convection to the Labrador 
Sea (Våge et al. (2009) and Yashayaev and Loder (2009)). The beginning of the trend reversal was marked by very energetic fluctuations in the velocity near the bottom of the deeper moorings. The frequency and vertical trapping of these motions are typical of topographic Rossby waves, and could be part of the fast response to the thermohaline forcing at the Labrador Sea. The response to changes in the thermohaline forcing in the Nordic seas was described as a two-stage process by Gerdes and Koberle (1995). The first stage is a fast response induced by coastally trapped waves (with characteristics typical of TRW) propagating away from the source region. It is followed by a much slower response during the second stage that is caused by the advection of the modified dense water properties. The gradual trend and the rapid response we see at Line $\mathrm{W}$ could manifest this type of two-stage adjustment. However, if this is the case, our record is not sufficiently long to observe the slow advective response, which based on the arrival times of the LSW that was ventilated in the mid 90s and early 2000s described in Chapter 3, could take in the range of 6-9 years. 


\section{Chapter 5}

\section{The response of the circulation to deep potential vorticity anomalies:}

A PV-inversion approach with applications to the North Atlantic DWBC

\section{$5.1 \quad$ Introduction}

The North Atlantic Deep Western Boundary Current (DWBC) originates at the exit of the Nordic seas, where the densest of the North Atlantic Deep Water components are formed (McCartney (1992)). As the water spills over the sills, it entrains large amounts of surrounding water. The properties of the final product of the overflows that reaches the Labrador Sea via the DWBC will therefore depend on both the air sea fluxes in the Nordic Seas that control the intensity of convection, as well as the properties of the water that is entrained during the sinking (eg. Swift et al. (1980) and Bacon (1998)). 
As the DWBC circulates around the Labrador Sea, the contribution from the Labrador Sea Water (LSW) happens through the entrainment of LSW into the Overflow Water (OW) at depth, as well as via direct export of LSW at intermediate depths. The properties of the core of the LSW are, as for the OW, related to the atmospheric forcing. Two types of LSW are formed in the Labrador Basin. During severe winter conditions convection can penetrate to $2000 \mathrm{~m}$ and a thick homogeneous layer of dense water is formed, typically referred to as 'Classical' or deep LSW (dLSW). During milder winters convection does not reach as deep, forming a thinner, less-dense mode known as upper LSW (uLSW) (eg. Yashayaev (2007)). Some of this water formed in the Labrador Sea is eventually exported into the subtropical domain via the Deep Western Boundary Current. Changes in the depth of convection in the Labrador Sea will have a clear signature in the potential vorticity (PV) field along the DWBC (Talley and McCartney (1982)), with recently ventilated LSW, of either type (deep or upper) characterized by negative PV anomalies. Typically, only one of the two types of LSW is formed annually, thus negative PV anomalies in the uLSW tend to be accompanied by positive anomalies in the dLSW and vice versa. Evidence of this alternation between upper and deep LSW was provided in Chapter 3.

Changing conditions at the Nordic and Labrador Seas have therefore a direct effect in the density structure of the DWBC. Waters that have been recently ventilated at either site have lower PV than the surrounding water. Once the waters sink and are isolated from the surface, this low PV signal remains relatively unchanged (with the exception of mixing with high PV surrounding water), and it is advected along the western boundary into the subtropics by the DWBC.

The effect of the changing conditions at the dense water formation regions in the circulation of the DWBC is, on the other hand, much less clear. Theory predicts that, in steady state, more dense water formation will result in larger transports in the DWBC (Stommel and Arons (1960)). However, the range in the responses of the DWBC flow to changing conditions in the Nordic and Labrador Basins obtained in modeling studies is rather large. In particular, the role that the formation of LSW 
plays in the Meridional Overturning Circulation (MOC) of the North Atlantic, is still uncertain. While some models find that enhanced dense water formation in the Labrador Sea leads to stronger overturning (eg. Mauritzen and Häkkinen (1999), Böning et al. (2006)), others find LSW production does not contribute to the MOC (Pickart and Spall (2007)), only the overflows do (Gerdes and Koberle (1995), Kohl and Stammer (2008)).

The analysis of 4 years of conductivity/temperature/pressure (CTD) and horizontal velocity measurements from the Line $\mathrm{W}$ moored array shed some light on this subject. In Chapter 4, we found that changes in the transport of the DWBC were in-phase with enlarged presence of OW rather than dLSW. However, the detailed structure of the velocity field within the DWBC revealed that at times when recently ventilated dLSW was present at Line W, (the dLSW having opposite phase than the OW) there was a small increase in the velocity of the DWBC at intermediate depths. The amplitude of this increase in the velocity as well as the fraction of the DWBC area that was affected by it were small, and its contribution to the total transport across the array was negligible.

Here, our goal is to explore theoretically how changes in the flow along the DWBC are associated with changes in the ventilation of the different components of the NADW as indicated by their PV distribution. In particular we ask: are PV anomalies of a particular sign associated with enhanced or decreased flow in the DWBC? And if so, what is controlling the strength of the overall transport of the DWBC? PV unlike chemical tracers such as CFCs or oxygen, is a dynamical tracer, meaning that some information about the circulation itself is contained in the PV distribution. In the particular case of PV, all the information about the flow is contained in its distribution. This implies that given a PV distribution, and provided some additional information, one can recovered the full velocity field. This is often referred to as the PV invertibility principle (Hoskins et al. (1985)). Inverting PV is a diagnostic (not a prognostic) tool, often used in the atmospheric community. Although it is much less frequent among oceanographers, some examples, most of them in the context of the 
quasigeostrophic dynamics, can be found as well. In this chapter, we invert isolated PV anomalies associated with the DWBC different water masses, to recover the individual contributions to the flow anomalies induced by each of them. Assuming the dynamics are linear, the complete flow associated with a given PV anomaly field can be reconstructed by superimposing the solutions obtained from the individual anomalies. We find that both enhanced LSW production and enhanced OW production lead to the strengthening of the DWBC. The response to the different water masses is not independent, but it is connected through the atmospheric forcing and the exchanges between the Labrador Sea and the Greenland-Iceland-Norwegian (GIN) Seas where the deep water is formed. The overall response that we see at mid-latitudes at Line $\mathrm{W}$, that integrates all the different contributions, is mostly driven by changes in the deep OW rather than by changes at intermediate depths.

\subsection{The machinery: a PV inversion.}

The invertibility principle of potential vorticity states that at any given time, all the dynamical information about the flow, namely the velocity and density fields, can be deduced from the distribution of potential vorticity. However, to be able to recover both velocity and density separately, some additional information needs to be provided (Hoskins et al. (1985)). This additional information is in the form of: (1) a balance condition (i.e. a relation between the density and the velocity field), (2) some type of reference state (a spatial or temporal mean) and (3) boundary conditions appropriate to the problem.

The invertibility principle holds even when friction and diabatic processes are important, and it is as accurate as the balance condition itself (how close to balanced the total flow is). There are a number of choices for balance conditions (see for instance McIntyre (2003)), of which the geostrophic balance is the simplest. Detailed descriptions of the balanced flow relations and PV dynamics that apply to different types of motions can be found in Vallis (1996). 
The inversion of PV itself is only a diagnostic tool, but in combination with a prognostic equation, such is provided by the conservation of $\mathrm{PV}$, it has been used to characterize the evolution of a flow (eg. Davis and Emanuel (1991) for the case of cyclones). In the oceanographic literature, the use of the PV invertibility principle is far less common, with the exception of quasigeostrophic (QG) dynamics (eg. Lapeyre and Klein (2006)). Next, we derive an approximate form of Ertel PV by applying scalings similar to those in the continuously stratified QG case and that, in the limit of constant stratification and no variations in the Coriolis parameter, takes the same form as the QG pseudopotential vorticity for the ocean.

\subsubsection{A simplified PV equation.}

The general form of Ertel PV for the ocean is:

$$
q=-\omega_{a} \cdot \frac{\nabla \rho}{\rho}
$$

Where $\omega_{a}$ is the three dimensional absolute vorticity of the fluid parcel, and $\rho$ is the density of the fluid. Along the path of the DWBC in the North Atlantic, the flow is strongly steered by the continental slope, and streamlines tend to follow isobaths. So the natural axes of the problem are the along- and across-isobath directions. After rotating the coordinate system, the approximate form of the EPV may be obtained by neglecting the vertical velocity as compared to the much larger horizontal velocities:

$$
q=-\frac{1}{\rho_{0}}\left\{-\frac{\partial v}{\partial z} \frac{\partial \rho}{\partial x}+\frac{\partial u}{\partial z} \frac{\partial \rho}{\partial y}+\left[\frac{\partial v}{\partial x}-\frac{\partial u}{\partial y}+f\right] \frac{\partial \rho}{\partial z}\right\}
$$

where $x$ is the along-isobath direction, positive to the east-northeast (upstream in the DWBC), $y$ is the across-isobath direction, positive to the north-northwest, and $z$ is vertical coordinate, positive upward. 
Here we focus in the 2-dimensional problem, and assume that all changes in the along-isobath direction are negligible:

$$
q \cong-\frac{1}{\rho_{0}}\left\{\frac{\partial u}{\partial z} \frac{\partial \rho}{\partial y}+\left[-\frac{\partial u}{\partial y}+f\right] \frac{\partial \rho}{\partial z}\right\}
$$

Next, we separate the fields into a mean and a perturbation:

$$
\begin{array}{r}
u=\bar{U}(y, z)+u^{\prime}(y, z, t) \\
\rho=\bar{\rho}(y, z)+\rho^{\prime}(y, z, t)
\end{array}
$$

For the purpose of this work the means $\bar{U}$, and $\bar{\rho}$ are temporal means representing the background state, and are assumed to be larger than the perturbations $u^{\prime}$ and $\rho^{\prime}$. This assumption is true for the low frequency part of the fields; based on the observations presented in Chapters 3 and 4, both fluctuations in the velocity and density modes that characterize the slowly-varying part of the fields have smaller magnitude than the respective means. Note that the time-dependence of the perturbations is irrelevant here, since the inversion is only used as a diagnostic tool, not combined with a prognostic equation.

Substituting in equation 5.3, and rearranging we obtain:

$$
\begin{aligned}
g q^{\prime}= & -\frac{g}{\rho_{0}}\left\{\frac{\partial \bar{U}}{\partial z} \frac{\partial \rho^{\prime}}{\partial y}+\frac{\partial u^{\prime}}{\partial z} \frac{\partial \bar{\rho}}{\partial y}+\frac{\partial u^{\prime}}{\partial z} \frac{\partial \rho^{\prime}}{\partial y}\right\} \\
& -\frac{g}{\rho_{0}} \frac{\partial \rho^{\prime}}{\partial z}\left(-\frac{\partial \bar{U}}{\partial y}+f\right)+\frac{g}{\rho_{0}} \frac{\partial \rho^{\prime}}{\partial z} \frac{\partial u^{\prime}}{\partial y}-N^{2} \frac{\partial u^{\prime}}{\partial y}
\end{aligned}
$$


Where

$$
N^{2}=-\frac{g}{\rho_{0}} \frac{\partial \rho_{0}}{\partial z}
$$

has been used.

The first condition for the invertibility principle requires that the circulation is in balance. The simplest possible condition is that the flow is in geostrophic balance. For the ocean, and in particular for large-scale flows, thermal wind provides an accurate representation of the total flow. This allows us to introduce the geostrophic streamfunction $\phi$ :

$$
\begin{array}{r}
u^{\prime}=-\frac{1}{f \rho_{0}} \frac{\partial \phi}{\partial y} \\
\frac{\partial \phi}{\partial z}=-g \rho^{\prime}
\end{array}
$$

and rewrite the equation as follows:

$$
\begin{aligned}
g q^{\prime}= & \frac{1}{\rho_{0}}\left\{\frac{N^{2}}{f} \frac{\partial^{2} \phi}{\partial y^{2}}+\left(-\frac{\partial \bar{U}}{\partial y}+f\right) \frac{\partial^{2} \phi}{\partial z^{2}}\right\} \\
& +\frac{1}{\rho_{0}}\left\{\left(\frac{g}{f \rho_{0}} \frac{\partial \bar{\rho}}{\partial y}+\frac{\partial \bar{U}}{\partial z}\right) \frac{\partial^{2} \phi}{\partial y \partial z}-\frac{N^{2} \hat{\beta}_{y}}{f^{2}} \frac{\partial \phi}{\partial y}\right\} \\
& -\frac{1}{f \rho_{0}^{2}}\left\{\left(\frac{\partial^{2} \phi}{\partial y \partial z}\right)^{2}-\frac{\partial^{2} \phi}{\partial y^{2}} \frac{\partial^{2} \phi}{\partial z^{2}}+\frac{\hat{\beta}_{y}}{f} \frac{\partial \phi}{\partial y} \frac{\partial^{2} \phi}{\partial z^{2}}\right\}
\end{aligned}
$$


where

$$
\hat{\beta}_{y}=\frac{\partial f}{\partial y}=\beta \cos (\alpha)
$$

is the derivative of the Coriolis parameter in a coordinate system rotated $\alpha$ degrees counterclockwise.

The first and second lines in equation 5.5 contain all of the linear terms, and the third line contains the non-linear terms. The coefficients of the cross-derivative term in the second line scales with the ageostrophic component of the background velocity field. As long as the mean flow is in thermal wind balance, it can be neglected. In the case of constant $f$, the first derivative terms also appearing in the second line can be neglected as well. The latter is a reasonable assumption for our two dimensional problem, since the lateral extent of the DWBC does not typically exceed $200 \mathrm{~km}$. The simplification of the cross-derivative terms will be justified in the next section.

For the linear problem in the absence of a background velocity, $\bar{U}=0$, and setting $\hat{\beta}_{y}=0$, equation 5.5 reduces to:

$$
q^{\prime}=\frac{1}{\rho_{0}}\left(\frac{\partial^{2} \phi}{\partial y^{2}}+\frac{f^{2}}{N^{2}} \frac{\partial^{2} \phi}{\partial z^{2}}\right)
$$

were the PV perturbation, $q^{\prime}$, now has been rescaled (divided) by the background $\mathrm{PV}, \bar{q} / f^{2}\left(\bar{q}=-\left(f / \rho_{0}\right) \partial \bar{\rho} / \partial z\right)$. The potential vorticity represented in this equation is different from the QG pseudopotential vorticity, but related to it (Charney and Stern (1962)):

$$
q_{p}^{\prime}=\frac{1}{\rho_{0}}\left(\frac{\partial^{2} \phi}{\partial y^{2}}+\frac{\partial}{\partial z}\left(\frac{f^{2}}{N^{2}} \frac{\partial \phi}{\partial z}\right)\right)
$$

While QGPV is conserved following the horizontal flow, equation 5.6, derived from 


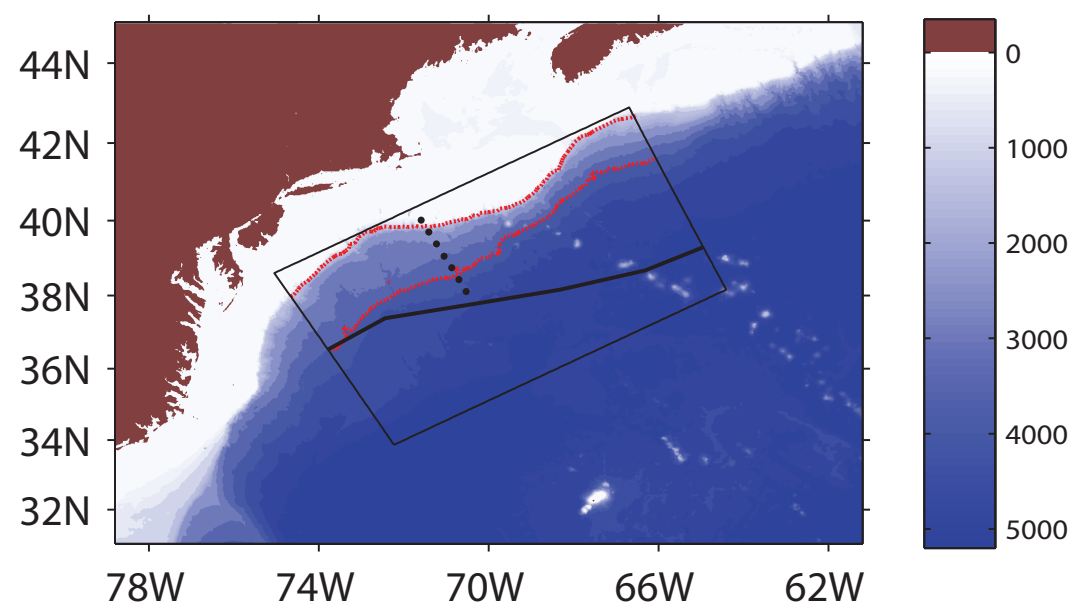

Figure 5-1: Map of the area of study. The location of the Line W observations is shown by the dotted line. The red contours show the $1500 \mathrm{~m}$ and $3000 \mathrm{~m}$ isobaths. The black line shows the mean path of the Gulf Stream inferred from altimetry.

making similar approximations to those in QG theory, is conserved when following the full three-dimensional flow.

\subsubsection{The elements of the problem: an idealized representa- tion of the western North Atlantic}

In order to solve for the streamfunction, $\phi$, in equation 5.5, two things required are: the potential vorticity distribution, which appears in the left hand side of the equation, and the reference state, in the coefficients that precede the vertical derivative. In this section we describe idealized PV perturbations and background fields that are meant to represent the conditions in the western North Atlantic along the DWBC between Grand Banks and the Gulf Stream/DWBC cross-over at Cape Hatteras, based on the Line W observations (figure 5-1). We will show that our particular choice of reference state justifies some of the simplifications made to get from equation 5.5 to 5.6.

\section{The background state and the balance condition}

The structure of the idealized background fields was based on CTD and direct velocity measurements from a series of repeat hydrographic sections at Line $\mathrm{W}$ (ca. $69^{\circ} \mathrm{W}$ and 
(a)

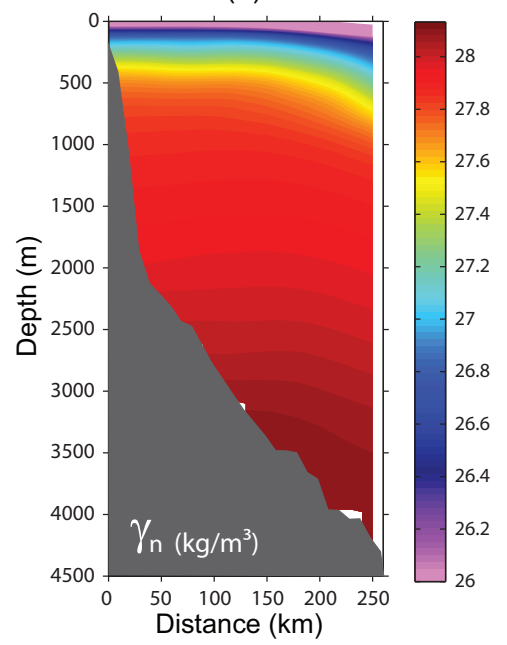

(b)

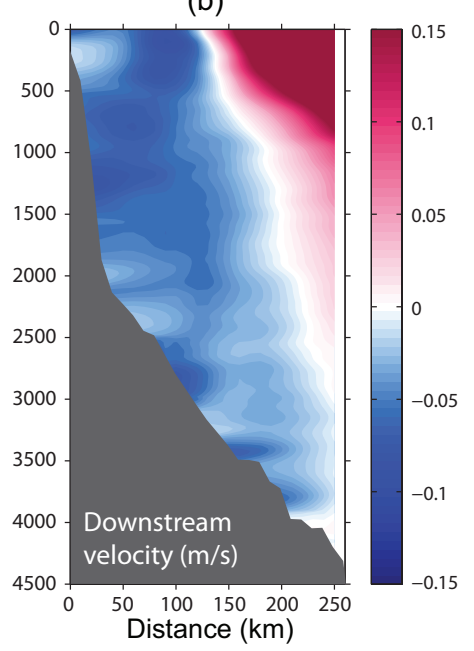

(c)

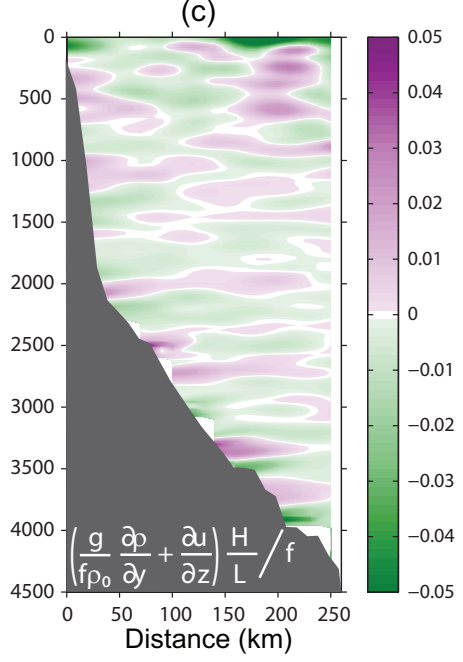

Figure 5-2: Mean neutral density (a) and downstream velocity (b) sections inferred from a total of 13 repeat hydrographic sections at $69^{\circ} \mathrm{W} 40^{\circ} \mathrm{N}$. Panel (c) shows the ratio, between the thermal wind residual, coefficient in the cross-derivative term in equation 5.5, calculated from (a) and (b) and the Coriolis parameter, $f$, that scales the vertical derivative in the Laplacian. This ratio has been scaled by the aspect ratio of the problem $(\mathrm{H}=1000 \mathrm{~m}$ and $\mathrm{L}=100 \mathrm{~km})$ to account for the difference in the derivatives.

$40^{\circ} \mathrm{N}$, details provided in Chapter 2, section 2.2.2). Over the continental slope, the mean density distribution (figure 5-2a) is characterized by a region of relative flat isopycnals. The stratification in the upper $1000 \mathrm{~m}$ of the water column is one order of magnitude larger than at deeper levels, with typical values for the Brunt-Väisälä frequency, $N^{2}$, of $10^{-6} \mathrm{~S}^{-2}$ at thermocline levels, and $10^{-7} \mathrm{~S}^{-2}$ and smaller in the deep water. Departures from the section's mean $N^{2}(z)$ profile are small within the Slope Water (not the case in the Gulf Stream), so there is little loss of generality in assuming there are no horizontal variations in the stratification. The mean velocity field (figure $5-2 \mathrm{~b}$ ) is relatively uniform with values of several $\mathrm{cm} / \mathrm{s}$. The contribution of the relative vorticity of the mean DWBC is second order compared to $f$ (also not true in the Gulf Stream region), and so $\partial \bar{U} / \partial y$ may be neglected. As for the cross-derivative term in equation 5.5, we find that thermal wind provides an accurate representation of the mean downstream velocity (figure 5-2c) and the contribution from the ageostrophic component of the background velocity field can also be neglected. 
Regarding the non-linear terms (last line in equation 5.5) our approach was to neglect them first, solve for the linear problem, and evaluate those neglected terms a posteriori. If their contribution was significant, they could be added to the left hand side of the equation, and the problem iterated until covergence was reached. However, this was not neccesary, since in all the experiments we carried out, the non-linear terms were at least two orders of magnitude smaller than the linear terms.

\section{The PV perturbation.}

Changes in the forcing in the Labrador and Nordic Seas led to changes in the properties of the waters that are formed and then exported along the DWBC to midlatitudes. As it was discussed in Chapter 4, the signature of these changes in the different water masses is especially clear in the PV fields along the DWBC. The PV anomalies we observed consist of alternating layers of negative and positive anomalies, with the uLSW and the OW in-phase, and opposite phase for the dLSW (figure 5-3a). Throughout the record, the sign of the anomalies reverses frequently, but we observed that on average during the early 2000s the negative PV anomalies were dominant in the dLSW depth range, while in the later part of the decade, the uLSW and the OW had lower PV than the mean (figure 5-3b).

Our idealized version of this PV distribution inferred from the observations, consists of three individual PV anomalies: an upper anomaly centered between 500 and $1000 \mathrm{~m}$ representing the uLSW, an intermediate anomaly roughly contained between $1000 \mathrm{~m}$ and $2500 \mathrm{~m}$ representing the dLSW, and a deep anomaly between approximately 2500 and $4000 \mathrm{~m}$. The vertical and horizontal structure of these anomalies was prescribed by a Gaussian distribution with peak amplitude, width and cutoff lengths inferred from the observations. In the particular case of the uLSW, the Line W moored array does not fully resolve the anomalies, so we assumed a distribution that was vertically symmetric and had similar lateral extension as the dLSW below. The solution is not sensitive to the specific details of these idealized anomalies, since the elliptical operator filters out the small scale structure in the PV distribution; only 

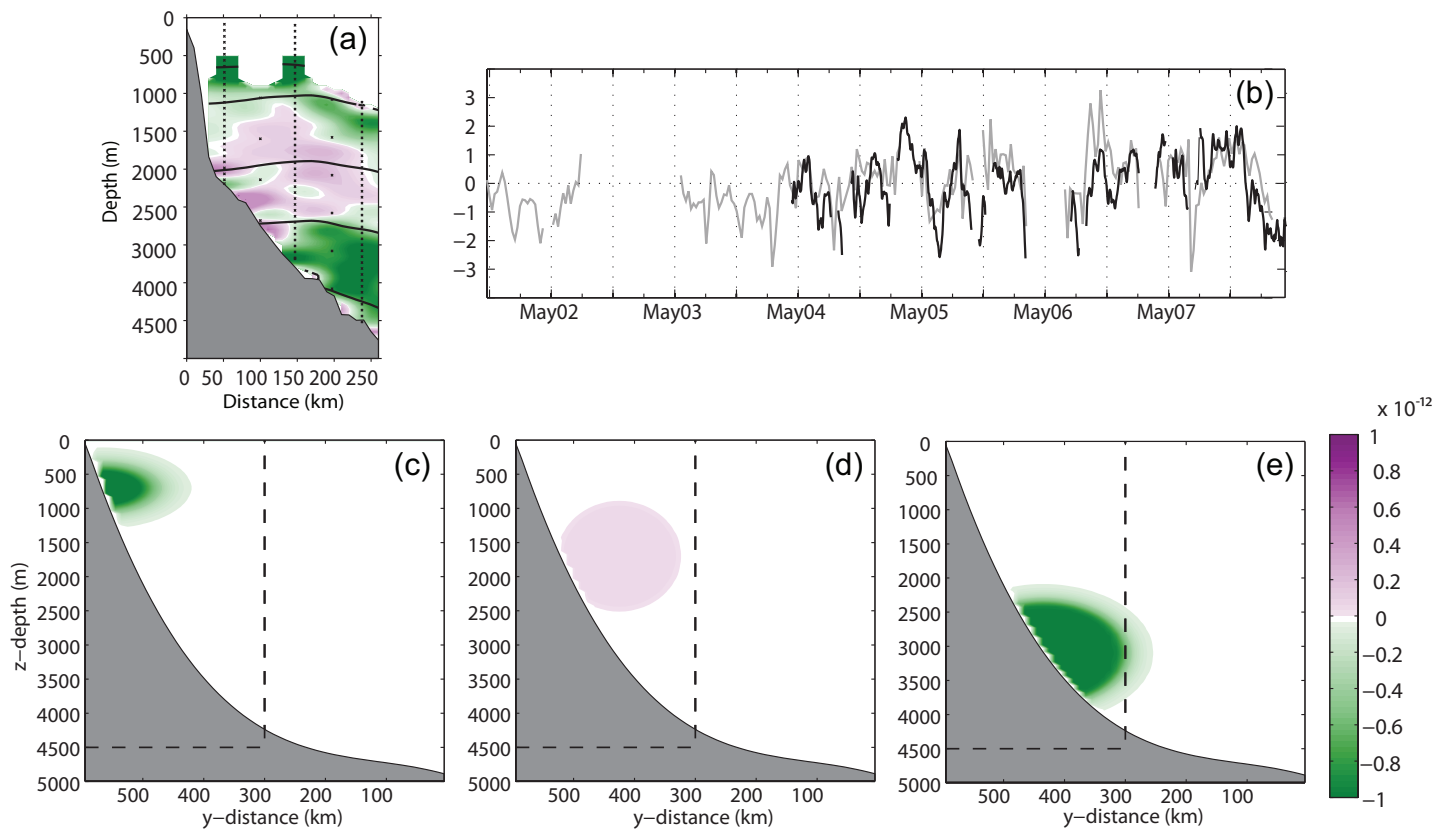

Figure 5-3: Panel (a) shows the spatial pattern of the leading EOF of the potential vorticity, here with units of $(\mathrm{ms})^{-1}$, from the 4-year Line W moored array (Chapter 4). Panel (b) shows, in black, the associated principal component and in gray, the principal component associated with the equivalent leading EOF of the central mooring in the array (longer record, Chapter 3). Panels (c), (d) and (e) show the idealized domain with the modeled uLSW, dLSW and OW PV anomalies, respectively.

large scale features matter.

\subsection{An infinite basin with constant stratification}

The simplest of the problems considered, yet a meaningful one, is the case of an infinite $f$-plane basin with constant stratification under linear dynamics. The $f$-plane and linear dynamics assumptions will be made throughout the chapter, the infinite domain and constant stratification will be relaxed in later sections. Under these assumptions, equation 5.6 can be solved analytically by stretching the vertical coordinate introducing a new variable, $\xi$ :

$$
\xi=\left(\frac{f}{N}\right) z
$$


Doing so, equation 5.6 is transformed into the Poisson equation in two dimensions:

$$
S(y, \xi)=\frac{\partial^{2} \phi}{\partial y^{2}}+\frac{\partial^{2} \phi}{\partial \xi^{2}}
$$

with the source term, $S(y, \xi)$, given by:

$$
S(y, \xi)=\frac{g \rho_{0} f}{N^{2}} q^{\prime}
$$

A solution to the Poisson equation for an infinite domain that is azimuthally symmetric around the source is:

$$
\phi(y, \xi)=\iint S\left(y_{0}, \xi_{0}\right) G\left(y, \xi ; y_{0}, \xi_{0}\right) d y_{0} d \xi_{0}
$$

where $G\left(y, \xi ; y_{0}, \xi_{0}\right)$ is the Green's function solution for the Poisson equation with a point source located at $\left(y_{0}, \xi_{0}\right)$, of amplitude 1 :

$$
G\left(y, \xi ; y_{0}, \xi_{0}\right)=\frac{1}{2 \pi} \ln \left(\sqrt{\left(\xi-\xi_{0}\right)^{2}+\left(y-y_{0}\right)^{2}}\right)
$$

Changing back to the original vertical coordinate, the final solution is:

$$
\phi(y, z)=\iint \frac{g \rho_{0} f}{N^{2}} q^{\prime}\left(y_{0}, z_{0}\right)\left[\frac{1}{2 \pi} \ln \left(\sqrt{f^{2} / N^{2}\left(z-z_{0}\right)^{2}+\left(y-y_{0}\right)^{2}}\right)\right] d y_{0} d z_{0}(5.1
$$

The velocity perturbation induced by a symmetric PV anomaly (see figure 5-4d), is symmetric as well, and has the same sense as the anomaly. A negative PV anomaly would have an associated anticyclonic flow, while a positive anomaly will produce a cyclonic circulation. For a typical ocean basin size, the solution has practically 

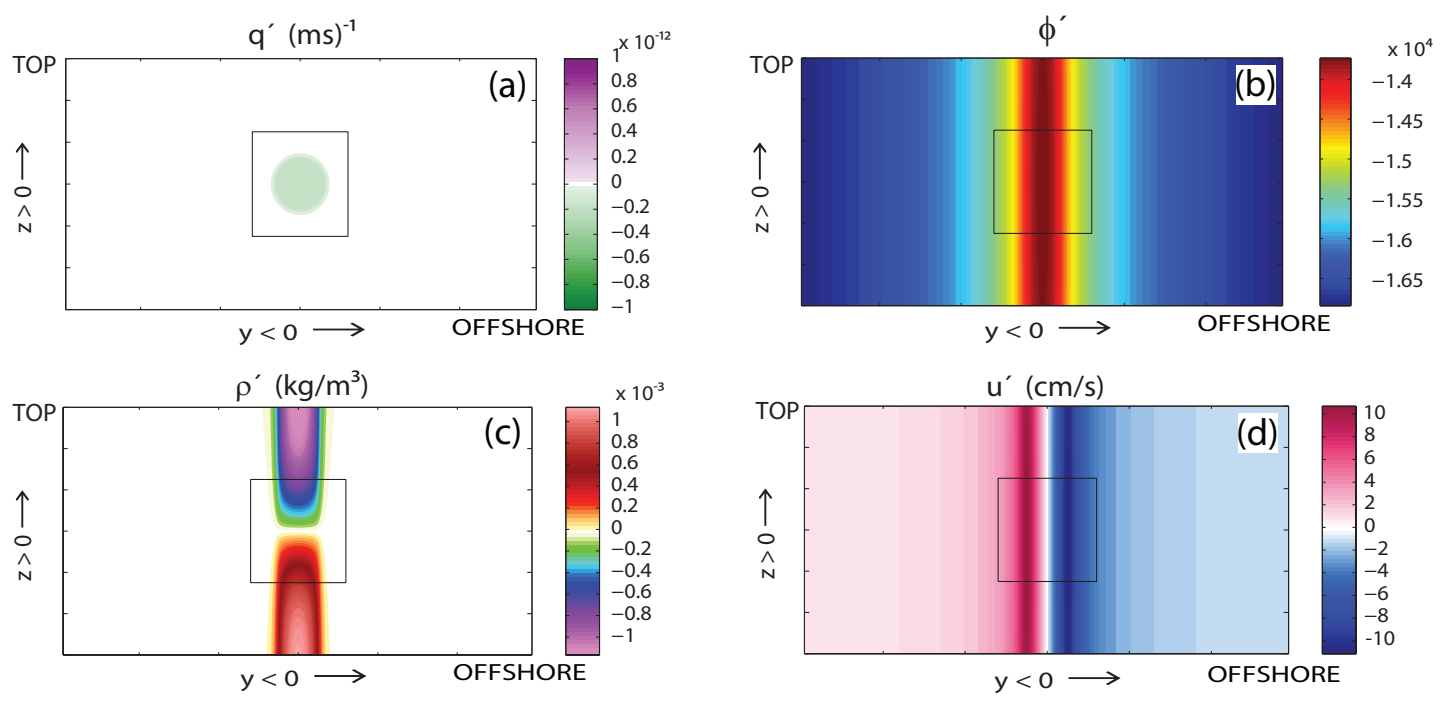

Figure 5-4: Analytical solution (b) and associated density (c) and velocity (d) perturbations for a negative PV anomaly (a) in an infinite basin. The solution is displayed for a region of dimensions $3000 \mathrm{~km} \times 15000 \mathrm{~m}$. A box, centered around the $\mathrm{PV}$ anomaly, and with dimensions similar to those used in the finite domain problem $(600 \mathrm{~km} \times 5000 \mathrm{~m})$ is also shown for reference.

zero vertical shear. The parameter that controls the vertical extent of these induced density and velocity perturbations is the background stratification, $N^{2}$. If we consider the solution for a point source:

$$
\phi(y, z)=\frac{g \rho_{0} f}{N^{2}}\left[\frac{1}{2 \pi} \ln \left(\sqrt{f^{2} / N^{2}\left(z-z_{0}\right)^{2}+\left(y-y_{0}\right)^{2}}\right)\right]
$$

and look at the limit when the vertical derivative vanishes:

$$
\frac{\partial \phi}{\partial z}=\frac{z}{z^{2}+\frac{N^{2}}{f^{2}} y^{2}} \rightarrow 0
$$

we find that the density perturbation goes to zero as $z \rightarrow \infty$. The same applies to the velocity perturbation. 


$$
\frac{\partial \phi}{\partial y}=\frac{\frac{N}{f} y}{z^{2}+\frac{N^{2}}{f^{2}} y^{2}} \rightarrow 0
$$

In practice, these rather large vertical and horizontal scales mean that the presence of boundaries will have a profound effect on the solution. It will be shown that the tendency for negative PV anomalies to induce an anticyclonic circulation is somewhat modified in the presence of boundaries.

\subsection{The boundary value problem}

The last of the additional pieces of information required to solved the problem are the boundary conditions. Equation 5.6 is an elliptical equation, and as such the possible boundary conditions for the problem are: (1) Neumann type, the normal derivatives to the boundary are prescribed, or (2) Dirichlet, the streamfunction itself is prescribed. In order for the problem to be well-posed, conditions of the second type must be prescribed at least at one of the boundaries ${ }^{1}$. Based on this limitation, a physically reasonable choice of boundary conditions is the following: at the top and bottom boundaries, $z=L_{z}$ and $z=0$, the density perturbation is required to vanish (the derivative normal to the boundary is set to zero):

$$
\begin{gathered}
\frac{\partial \phi}{\partial z}=0 \quad \text { at } z=L_{z} \\
\frac{\partial \phi}{\partial z}=0 \quad \text { at } z=0
\end{gathered}
$$

This choice may seem excessively restrictive, however, there is no a priori reason why an isolated PV anomaly in the interior would have a surface expression in the density field (it would in the pressure); and example of this are Meddies (eg. Paillet

\footnotetext{
${ }^{1}$ A solution to the equation and all Neumann boundary conditions plus any constant is a solution too.
} 
et al. (2002)). Whether or not certain PV anomalies in the DWBC are accompanied by density anomalies at the surface of a particular sign (the data shows some suggestion that this is the case for uLSW PV anomalies), is a different issue. Some comments regarding this matter will be made later in section 5.5. Along the northern boundary, the vertical wall, no-slip conditions are imposed (also, the derivative normal to the boundary is prescribed):

$$
\frac{\partial \phi}{\partial y}=0 \quad \text { at } y=L_{y}
$$

And finally, along the southern side, at the open boundary, $y=0$, the density perturbation is assumed to be zero, based on the expectation that far away from the PV source, the isopycnals will come back to rest (to the background).

$$
\phi=0 \text { at } y=0
$$

In practice, this last boundary condition is imposed by assuming that the streamfunction is constant, which is the only Dirichlet type of condition (the one required to keep the problem from being ill-posed). For simplicity, and in the absence of any additional information, we assumed $\phi=0$.

One important aspect of this choice of boundary conditions, is that although density perturbations are not allowed at the surface and bottom of the model basin, pressure perturbations are. And this implies, that even thought a free surface elevation is not explicitly included in the PV equation, it is represented in the solution by the pressure perturbation at the surface. This pressure perturbation can be re-scaled using the hydrostatic relation, and converted into an elevation. It will be shown in the experiments in the following sections, that this free surface elevation or surface pressure term, allows for a barotropic response to changes in the interior PV. Note that there is no reference level where the velocities are set to zero, compared to the 
traditional geostrophic velocity calculation.

The domain where we solve the problem is a rectangular box $600 \mathrm{~km}$ wide, with the solid wall at $y=600 \mathrm{~km}$, on the left side as represented in all figures that follow, and the open boundary at $y=0$, on the right side. The PV anomalies are always located near the wall, at a distance of $300 \mathrm{~km}$ or more from the open boundary where the streamfunction is required to vanish. The maximum depth, and the bottom boundary for the flat bottom experiments, is $5000 \mathrm{~m}$. For the experiments with a sloping bottom, the adopted topography was constructed from the mean topographic slope within the Slope Water region in the western North Atlantic shown in figure 5-1. The continuous sloping topography was approximated with a staircase representation, and the boundary conditions at the bottom and at the wall applied on a cell-by-cell basis.

The equation plus boundary conditions are solved with a modified version of the preconditioned conjugate gradient solver that is part of the MITgcm (global circulation model; Marshall et al. (1997)).

\subsubsection{The constant stratification case}

The simplest of the boundary value problems is the constant stratification case solved in a flat bottom basin. The anomaly is located up against the lateral wall, centered around $1500 \mathrm{~m}$ with a thickness of approximately $1500 \mathrm{~m}$, and a width of approximately $200 \mathrm{~km}$. In this, as well as in the next section, we used a negative anomaly with amplitude $\left(10^{-12} \mathrm{~ms}^{-1}\right)$ that represents the conditions during the early $2000 \mathrm{~s}$, when recently ventilated dLSW was present at Line W. Later in section 5.4.3, the sign of the PV anomaly at intermediate depth is reversed, and combined with uLSW and OW PV anomalies, in order to represent the conditions that were dominant during the last

years in the Line $\mathrm{W}$ record. As for background stratification, we used $N^{2}=10^{-6} \mathrm{~s}^{-2}$, also typical of intermediate waters.

The density perturbation associated with the solution in figure 5-5 shows that the isopycnals spread to accomodate the PV anomaly just as in the analytical solution 

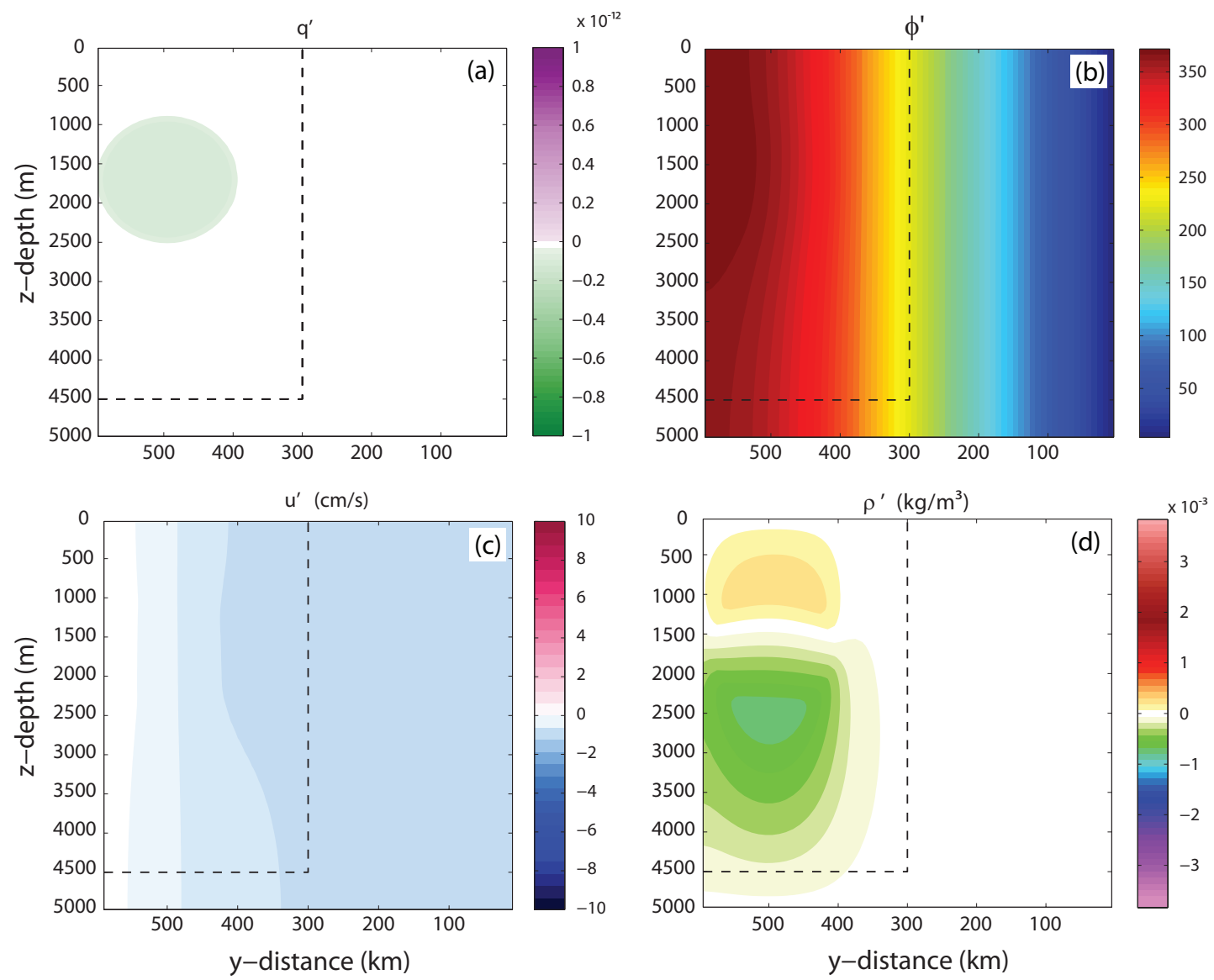

Figure 5-5: Negative dLSW PV anomaly (a) and corresponding solution (b) for a flat bottom basin with a stratification profile typical of the Slope Water. On the bottom, the circulation (c) and density (d) perturbations associated with the solution. The dashed box represents an area equivalent to the area of the Line $\mathrm{W}$ array.

in the infinite basin. The vertical asymmetry in the density perturbation, in this case, is driven by the boundary conditions. The position of the anomaly relative to the boundaries, where the density perturbation is required to vanish, forces the perturbation to decay faster towards the surface. The velocity field induced by the PV anomaly is negative, downstream in the DWBC sense. Its amplitude increases away from the wall, and it is maximum near the edge of the PV anomaly, where we find velocities of up to $2 \mathrm{~cm} / \mathrm{s}$. The sensitivity of the velocity field to the stratification is shown in figure 5-6 where we find the maximum velocity scales as the inverse of the stratification.

The amplitude of the velocity field response, is also proportional to the strength of 


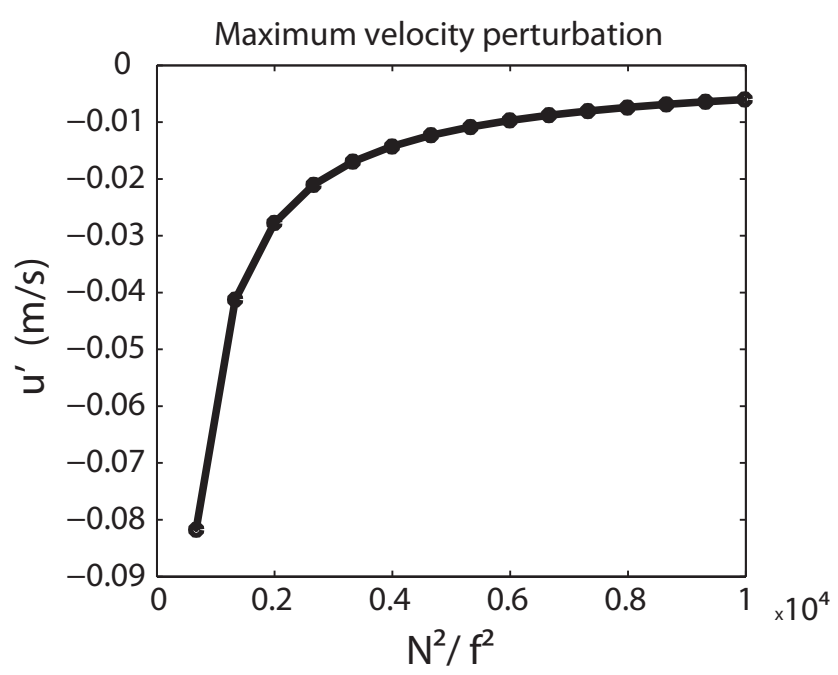

Figure 5-6: Maximum velocity (in $\mathrm{m} / \mathrm{s}$ ) as a function of the stratification for the flat bottom basin.

the PV perturbation, as expected for a linear problem. Away from the perturbation, as we move closer to the open boundary at $y=0$, the streamfunction becomes more barotropic, and decreases linearly with $y$, inducing a flow of constant strength all the way to the open boundary. This velocity at the open boundary is necessary to compensate for the PV perturbation in an integral sense. If we integrate the equation over area of the domain:

$$
\iint q^{\prime} d y d z=\frac{1}{\rho_{0}} \iint\left(\frac{\partial^{2} \phi}{\partial y^{2}}+\frac{f^{2}}{N^{2}} \frac{\partial^{2} \phi}{\partial z^{2}}\right) d y d z
$$

the contribution from $\partial \phi / \partial z$ is zero, by choice of boundary conditions (the total mass is redistributed but not changed), and we are left with the horizontal derivatives to balance the left hand side of the equation.

$$
\iint q^{\prime} d y d z=\frac{1}{\rho_{0}}\left(\left[\frac{\partial \phi}{\partial y}\right]_{y=L}-\left[\frac{\partial \phi}{\partial y}\right]_{y=0}\right)
$$

At the wall, $y=L$, the no-slip condition, $u(y=L)=0$, has no contribution either, 
leaving only the velocity at the open boundary to entirely compensate for the total change in PV. A negative PV anomaly will therefore induce a negative (out of the page) velocity at the open boundary. If one considers the problem from a transport perspective, this choice of boundary is somewhat artificial, since the transport will continue to increasing with increasing domain width. As we increase the domain size, the velocity at the open boundary remains constant, that is the horizontal gradient of pressure remains constant. In order for the horizonal pressure gradient to remain constant the pressure at the wall, where no-slip boundary conditions are imposed, increases proportionally to the increase in the domain width (figure 5-7). For a basin of infinite width, the transport integrated across the basin would therefore be infinite too. Allowing for a dense anomaly at the surface, which is equivalent to a positive PV sheet just below the surface (Bretherton (1966)), would partially alleviate this constraint at the open boundary. However, it is important to notice that despite this unrealistic feature of the solution, the predicted velocity in the vicinity of the PV anomaly where the solution is being compared to the observations $(300 \mathrm{~km}$ from the wall) is robust, independent from the domain size. The slope of the solution for any size domain is the same, it is the absolute value that is different.

Near the bottom boundary, in the region just off-shore from the edge of the PV anomaly, the velocity shows a slight decrease with depth. In a basin with constant stratification, this is due to the reference density field (not to the stratification itself). The background density profile is stable, meaning that the water below the perturbation is denser than the water above, $\rho_{b o t}>\rho_{t o p}$. When a density perturbation, $\rho^{\prime}$ with the same amplitude but opposite sign is added to this stable background, the change in density relative to the background, therefore the isopycnal slope, is less in the dense water at depth than in the lighter water above it.

$$
\frac{\rho_{b o t}+\rho^{\prime}}{\rho_{b o t}}<\frac{\rho_{t o p}+\rho^{\prime}}{\rho_{t o p}}
$$




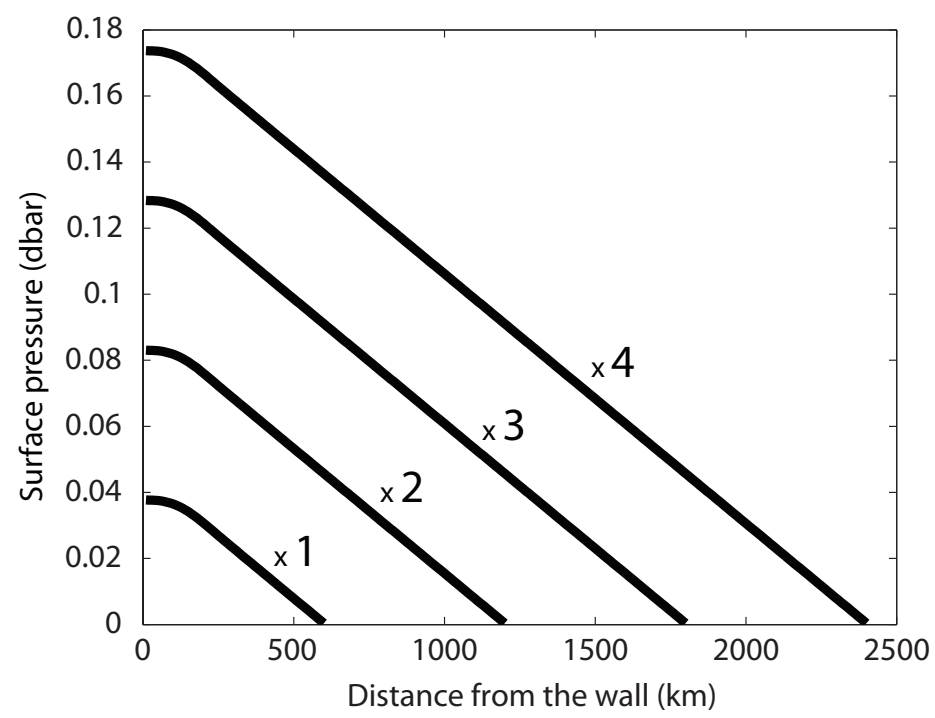

Figure 5-7: Pressure at the surface, expressed in decibars, for the solution in the original $600 \mathrm{~km}$ domain shown in figure $5-5$, and for the same problem solved in a domain that is 2, 3 and 4 times larger. The horizontal axis show distance from the wall where the no-slip condition is applied. The PV anomaly is located at $100 \mathrm{~km}$ from the wall in all four cases.

This is also true locally for cases when the stratification is not constant.

That was the solution for a flat bottom basin; does the sloping bottom play a role in the dynamics? For a 3 dimensional problem, when looking at the dynamics of the vertically integrated motions, the torque between the across-isobath changes in the bathymetry and along-isobath changes in the density field, the so-called Joint Effect of Baroclinicity and Relief (JEBAR, see Mertz and Wright (1992)), provides an important source of vorticity to the dynamics. In our case, however, there are no changes in the along-isobath direction, and so JEBAR has no contribution. The solution for the problem formulated exactly as before, but solved in a basin with a sloping bottom, is qualitatively the same as the flat bottom problem (figure 5-8). The largest differences in the solution are related to the horizontal penetration of the density perturbation. The presence of the boundary forces the density field to adjust further offshore, and the free surface over the sloping bottom has to elevate more to compensate for the changes in the total depth of the fluid (figure 5-9). The slightly 

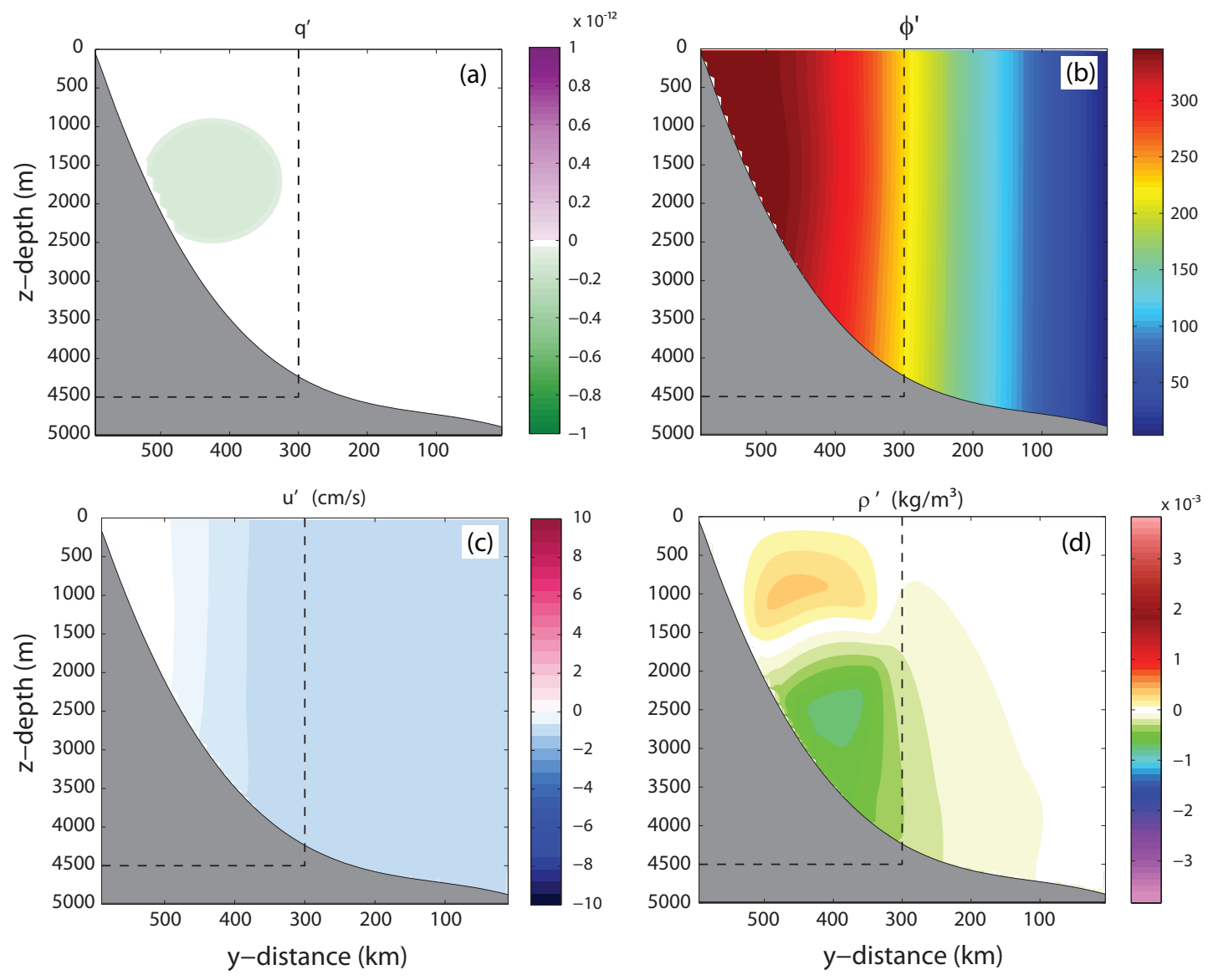

Figure 5-8: Negative dLSW PV anomaly (a) and corresponding solution (b) for a sloping bottom basin with constant stratification. On the bottom, the circulation (c) and density (d) perturbations associated with the solution. The dashed box represents an area equivalent to the area of the Line $\mathrm{W}$ array.

weaker velocities near the bottom that we found in the flat bottom case, are partially compensated here by the changes in the free surface elevation, leading to an even more barotropic solution.

\subsubsection{A Slope Water stratification profile}

When the constant stratification is replaced by the mean Slope Water $N^{2}$ profile from the Line $\mathrm{W}$ observations, the vertical structure of the solution changes, leading to important asymmetries both in the density and velocity distribution (figure 5-10). The density perturbation is now concentrated in the layers above the PV anomaly; 


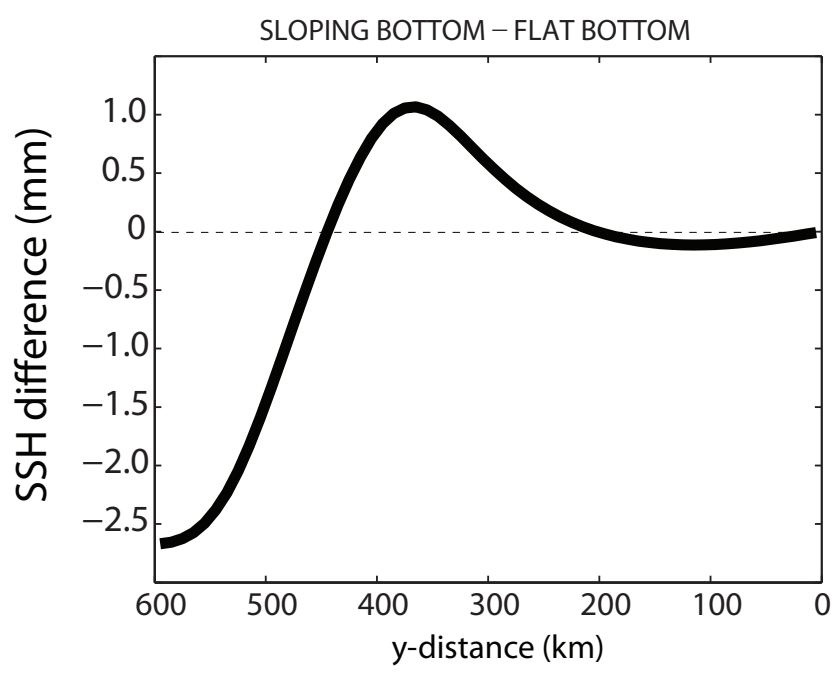

Figure 5-9: Difference between the sea surface height (converted from pressure using the hydrostatic relation) in the flat bottom and the sloping bottom solutions for a basin with constant stratification.

it is positive for a negative PV anomaly, meaning that, like for the mid-depth PV anomaly with constant stratification discussed before, dense fluid is being lifted up. The corresponding negative density perturbation below the anomaly is now much smaller. However, when added to the mean, the changes in the gradient of the total density field are comparable to those occurring up in the water column, pointing out that it is the ratio between background and perturbation that controls the amplitude of the response, not just either of them alone.

The velocity induced by the anomaly is still rather barotropic in nature, driven by the free surface elevation term. However, the velocity maximum is now located near the bottom at the offshore edge of the PV anomaly. This bottom intensification is due to the vertical structure of the background stratification. The larger stratification values in the upper part of the water column are effectively damping more of the velocity signal.

\subsubsection{The combined effect of LSW and OW PV anomalies}

In the previous examples we have seen that the effect of low PV anomalies in the dLSW can induced flow anomalies of -1 to $-2 \mathrm{~cm} / \mathrm{s}$, with a slight tendency for bottom 

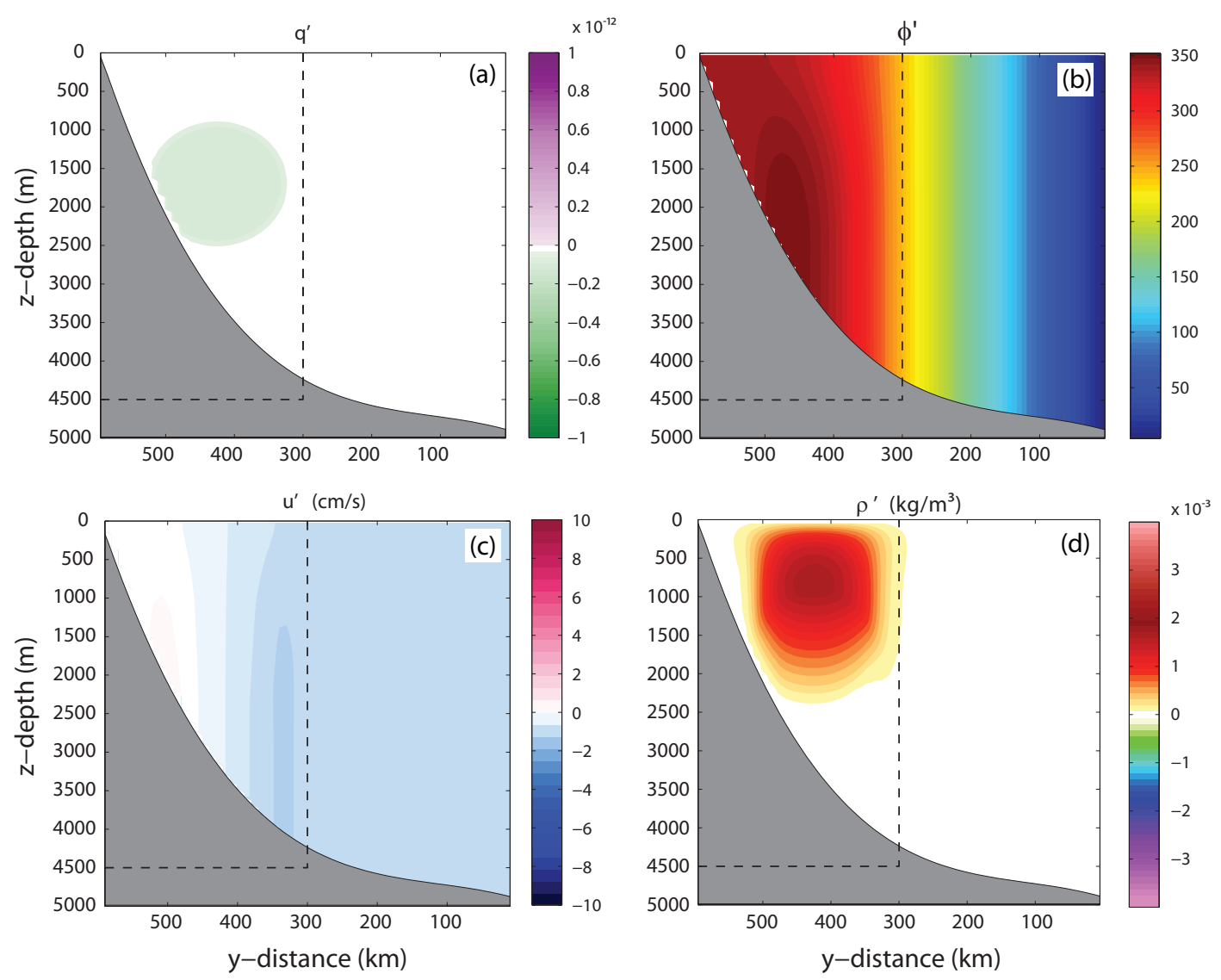

Figure 5-10: Negative dLSW PV anomaly (a) and corresponding solution (b) for a sloping bottom basin with a stratification profile typical of the Slope Water. On the bottom, the circulation (c) and density (d) perturbations associated with the solution. The dashed box represents an area equivalent to the area of the Line W array.

intensification due to the buffering effect of the background stratification. However, these PV anomalies in the dLSW depth range are not isolated. Observations showed that PV anomalies at intermediate depths are commonly accompanied by PV anomalies of opposite sign above and below them.

When we look at the contributions from individual LSW and OW anomalies (figure 5-11), we find that like the isolated dLSW anomalies, each of the anomalies contribute to the total circulation with their corresponding anticyclonic circulations (cyclonic for the positive dLSW PV anomaly) that are bottom intensified and reach their maximum values towards the edge of the anomaly. The total flow is mostly determined by the OW PV anomaly. The amplitude of the PV anomaly in the $\mathrm{OW}$ is larger than 
the dLSW, and so is the circulation induced by it. Additionally, the background stratification at the level of the $\mathrm{OW}$ is the weakest throughout the water column, and even having similar amplitudes, the OW PV anomaly would still induce a stronger circulation.

The density perturbation induced by the combined PV anomalies is positive (rising isopycnals), everywhere except for a small sector at the dLSW, a feature also seen in the observations. The bulk of the changes in the density field are due to the OW PV anomaly, which being right above the bottom can only displace dense water upwards, compared to the upper and deep LSW in which the density field below the PV adjusts too.

The patterns of the induced density and velocity perturbations compare well with the observations (figure 5-12). Scaling the density by the thermal expansion coefficient, $\alpha$, we find that most of the temperature change observed at Line $\mathrm{W}$ described in Chapter 4, is caused by the heaving of the isopycnals associated with the DSOW PV anomaly. The amplitude of the temperature change in the observations is slightly larger, probably due to the changes in temperature of the different water masses (changes within a density class) that we also found in the analysis of the central mooring at Line W (discussed in Chapter 3). The amplitude of the velocity perturbation inferred from the PV-inversion is also larger than that seen in the observations, and the detailed pattern slightly different, but overall the PV-inversion does a fair job capturing the large scale pattern.

The agreement between observations and inverted fields emphasizes supports our choice of boundary conditions. In the real ocean the individual PV anomalies we discussed here are not isolated, and some degree of compensation between upper, intermediate and deep PV anomalies exists. In adition, the Slope Water/DWBC system may not be considered as an open domain, but as a closed basin bounded by a solid lateral wall in one side, and by the Gulf Stream in the other. In that case, imposing no-slip conditions in the off-shore boundary, where the mean flow transitions from southwestward along the DWBC to northeastward along the Gulf Stream, may 

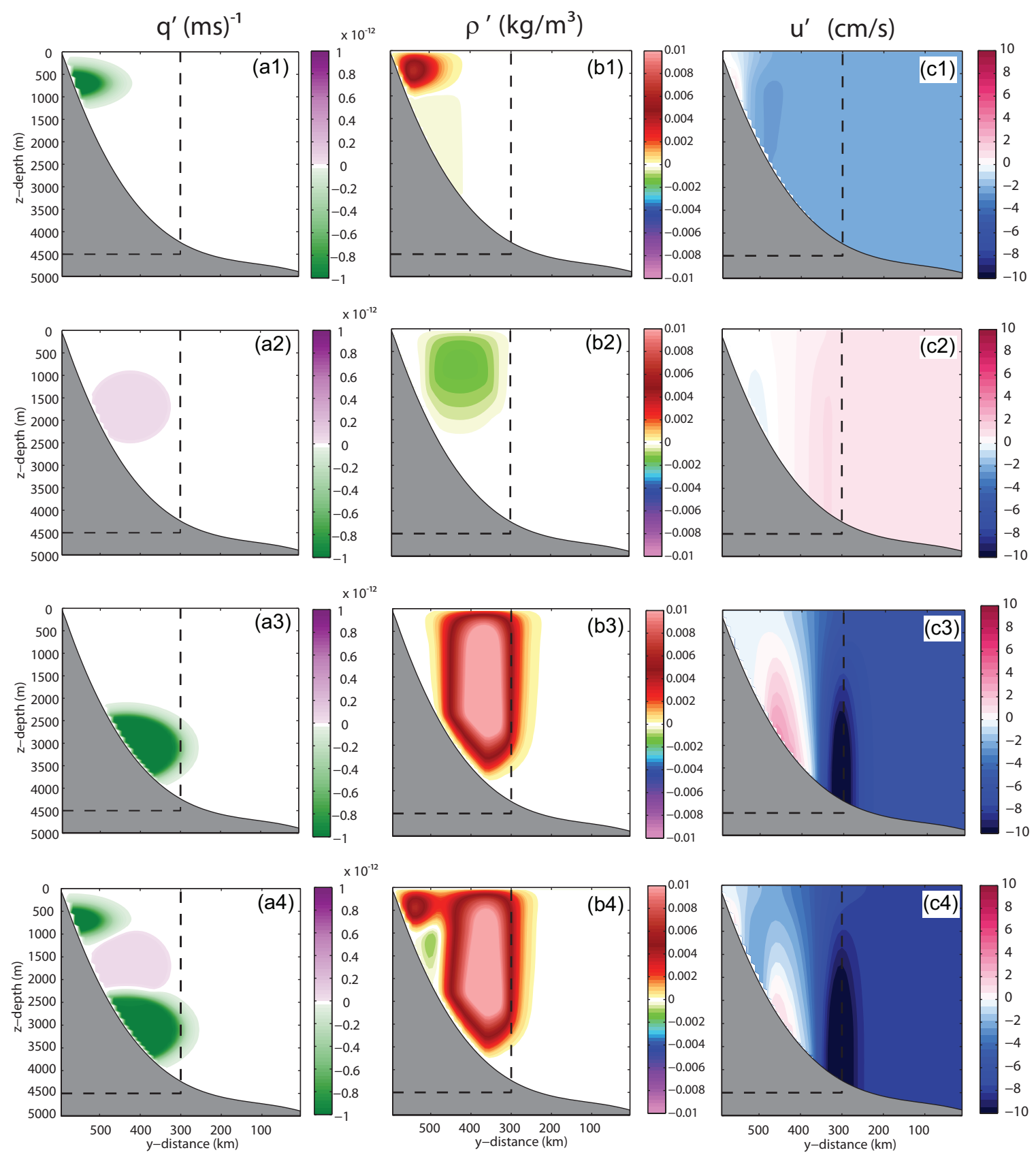

Figure 5-11: PV anomalies (a) and associated density (b) and velocity (c) perturbations for an uLSW (1), dLSW (2) and OW (3) anomalies, and for all of the above combined (4). 

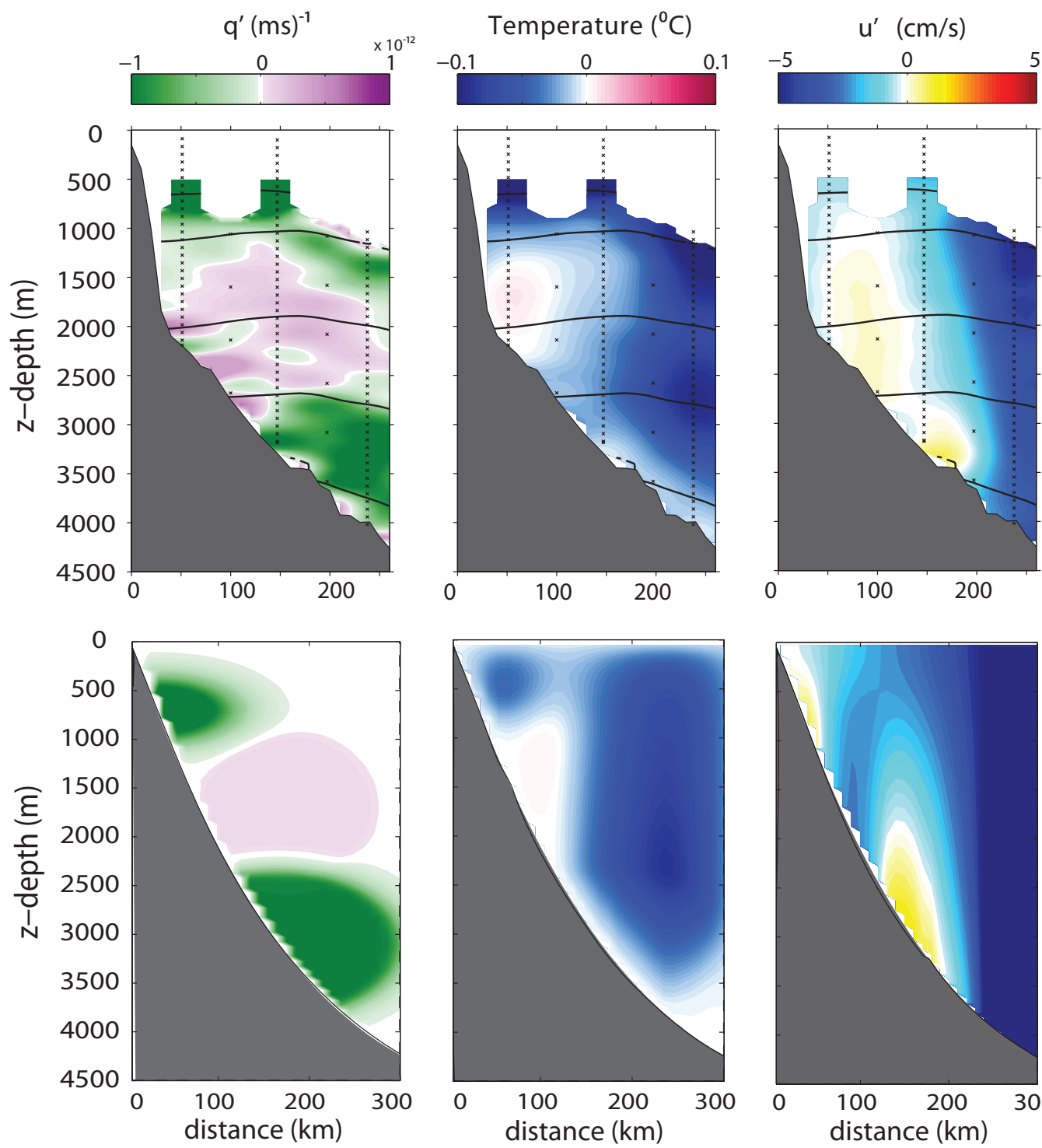

Figure 5-12: From left to right, the figures show PV anomalies, temperature pertubation (density scaled by the thermal expanssion coefficient) and velocity for the PV-inversion, on the bottom, and the observations, on top. The domain of the PVinversion has been cropped to the approximate location of the observations. The horizontal axis in the PV-inversion solutions has been inverted to match the observations. 
be more appropriate. However, such a boundary problem will be ill-posed, and no unique solution can be found. In the next section we explore the effect of adding a geostrophic baroclinic jet to the problem but keeping the same boundary conditions configuration.

\subsubsection{The effect of the mean Gulf Stream}

A more accurate representation of the conditions as the DWBC approaches the crossover should include the Gulf Stream. Here we represented the Gulf Stream as a geostrophically adjusted baroclinic jet, centered at 300km. Within the Gulf Stream the relative vorticity of the flow can account for as much as half of the total vorticity (Rossby and Gottlieb (1998)), and equation 5.6 needs to be accordingly modified to include it:

$$
g q^{\prime}=\frac{1}{\rho_{0}}\left\{\frac{N^{2}}{f} \frac{\partial^{2} \phi}{\partial y^{2}}+\left(-\frac{\partial \bar{U}}{\partial y}+f\right) \frac{\partial^{2} \phi}{\partial z^{2}}\right\}
$$

The solution to this new problem (figure 5-13) is qualitatively the same as before in the shallow region north of the jet. The largest difference south of the jet where the density perturbation is negative, meaning that the isopycnals are being pushed down. The combined effect of the isopycnals moving up north of the front and moving down south of it reinforces the jet, which is seen in the velocity perturbation as decrease in the negative flow with respect to the previous solution.

\subsection{Summary and discussion}

We explored the changes in the circulation in the North Atlantic DWBC associated with changes in the intermediate and deep water masses by means of a PV-inversion. We found that the anticyclonic circulation expected for a negative PV anomaly is slightly modified in the presence of boundaries. The positive flow in the anticyclonic 

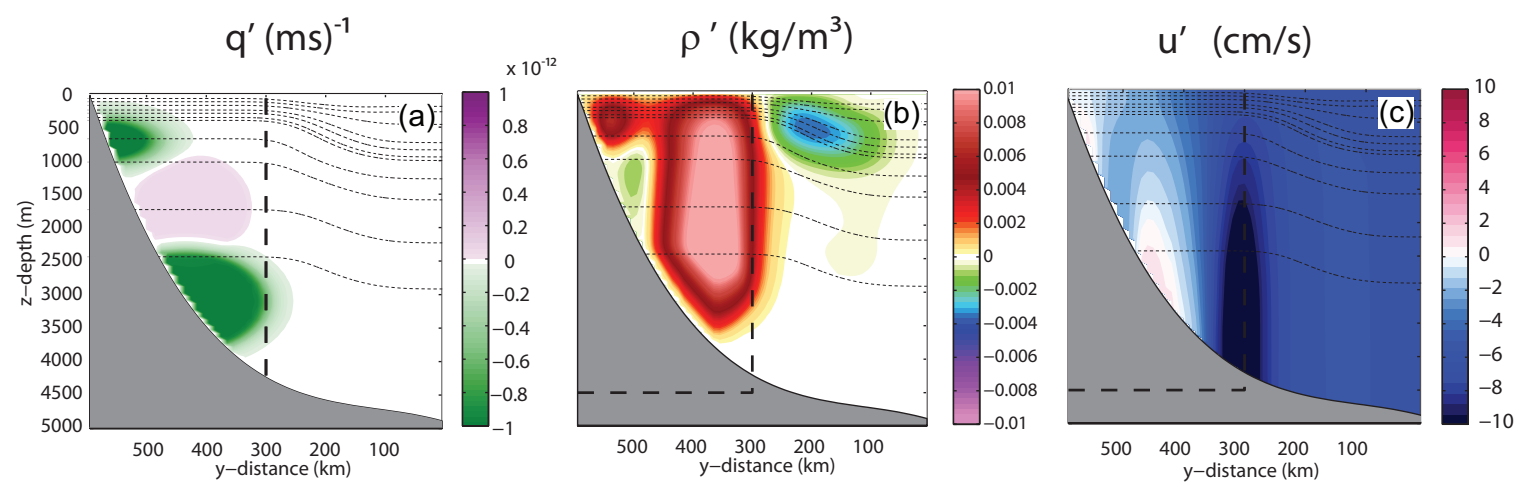

Figure 5-13: PV anomalies (a) and associated density (b) and velocity (c) perturbations induced by the combined uLSW, dLSW and OW PV anomalies combined in the presence of a geostrophically adjusted jet. The location of density front associated with the jet is shown by the black dotted contours.

pattern, is greatly reduced near a lateral wall, while the negative part of the flow extends all the way to the offshore open boundary. This distortion of the symmetry of the anticyclonic pattern implies an increase in the downstream velocity (in the DWBC sense) for a negative PV anomaly regardless of where in the water column it is located. The response, which is largely barotropic for PV anomalies typical of the Labrador Sea and Overflow waters, is slightly intensified near the bottom, where the background stratification is weaker, and therefore the slope of the isopycnals can experience larger changes, even for small perturbations in the density field. The response in the density field is larger in the upper part of the water column, and it is positive in the case of a negative PV anomaly, meaning that the redistribution of mass associated with the PV anomaly ocurrs mostly through isopycnal rising.

When positive and negative PV anomalies in the upper, intermediate and deep waters are combined to reproduce the vertical structure of the variability in the PV distribution inferred from observations, the resulting circulation is chiefly driven by the changes associated with the deep PV anomalies, dominantly from the OW. The flow near the bottom, at the base of the OW PV anomalies, can be as large as $-10 \mathrm{~cm} / \mathrm{s}$, consistent with the observed velocities as well. The contribution of the circulation induced by LSW PV anomalies is one order of magnitude smaller than the response associated with the OW. This in due to the amplitude of the PV anomaly itself, larger 
in the OW than in the LSW, and to the fact that the background stratification is smaller near the bottom allowing for a greater response.

A possible explanation as to why the amplitude of the OW PV anomalies is larger, is the origin of the anomalies themselves. The overflow from the Nordic Seas has a more direct route into the DWBC, which originates southeast of the sills (McCartney (1992)). Once in the Labrador Sea, the LSW joins the OW in the DWBC before continuing south. However, deep convection in the Labrador Sea takes places in the center of the basin, and a mechanism for the water to reach the boundary is required. In Straneo et al. (2003) the proposed mechanism involves both advective and diffusive processes. If that is the case, the amplitude of the signal that reaches the boundary, may be only a fraction of the original signal, possibly explaining why the PV anomalies in the LSW are much weaker than the OW anomalies at Line W.

The circulation patterns we inferred are strongly dependent on the choice of boundary conditions, implying that the amplitude of the model flows near boundaries should be considered qualitatively rather than quantitatively. For instance, the addition of density variations to the top boundary, such as colder water near the surface as is commonly observed coinciding with negative PV anomalies at the uLSW level, could relax the constraints in the pressure perturbations at the top boundary, and in the subsequent barotropic circulation associated with them. The effect of a positive density anomaly (a negative temperature anomaly) at the surface, is equivalent to having a thin layer of positive PV just below the surface (Bretherton (1966)). In the integral sense, this PV sheet will partially cancel the effect of a negative PV anomaly in the interior, and could significantly reduce, or even cancel, the flow at the open boundary.

Nevertheless, our results compare well with modeling studies that find that changes in the production of both LSW and OW can cause changes in the DWBC and ultimately the MOC. Böning et al. (2006) found that changes in the strength of convection in the Labrador Sea affected the deep transports in the boundary current within the basin, and this led to changes in the MOC of 1-2Sv. Changes of this magnitude could 
be obtained if the $1 \mathrm{~cm} / \mathrm{s}$ flow we inferred from the negative dLSW PV anomaly is applied over a width of $100 \mathrm{~km}$ and a depth of $1000 \mathrm{~m}$. In their model, Böning et al. (2006) did not include the overflows, so the LSW was the only source for changes in the deep water. Because the evolution of LSW and OW have opposite phase, it is possible that if both LSW and OW were included in that model, the effect of the LSW could be masked by the much larger control exerted by the OW.

Finally, the barotropic character of the response in the pressure field to changes in the PV in the interior implies that changes in the deep and intermediate water masses can be inferred from changes in Sea Surface Height (SSH). Using the hydrostatic relation, $P_{\text {surface }}=g \rho \eta$, a change in surface pressure across the slope of 0.01dbar (100Pa) obtained for the dLSW PV anomaly alone (figure 5-10b), corresponds to a change in SSH of approximately $1 \mathrm{~cm}$, a signal that in practice is not detectable given the accuracy of actual altimetric measurements $(2-3 \mathrm{~cm}$, Wunsch and Stammer (1998)). On the other hand, the amplitude of the surface pressure signal associated with the combined uLSW, dLSW and OW PV anomalies combined (not shown here) is much larger, as we said mostly due to the OW contribution, and corresponds to an SSH change across the slope of about $8 \mathrm{~cm}$, which should be detectable by the altimeter. 


\section{Chapter 6}

\section{Discussion}

In this thesis we have explored the variability in the circulation and hydrography of the western North Atlantic with special emphasis on the Deep Western Boundary Current. The main contribution of this thesis is in providing observational evidence of the connection between the variability in the subpolar and subarctic basins and the subtropics. This connection between high- and mid-latitudes takes the form of changes in the transport, properties and volumes of the intermediate and deep water masses that are exported along the DWBC. As these signals associated with changes in the water mass distribution advance through the western North Atlantic, they substantially affect the overall stratification in the region and can drive circulations that will either contribute to or slow down their own equatorward progression. Although the changes discussed here affect intermediate and deep waters, they have a signature in sea surface elevation and therefore in the velocity field of the upper ocean as well, that can ultimately interact with the wind driven circulation. In this section we highlight some of the specific findings from this thesis and relate them to what we know about the circulation of the North Atlantic in a more global context, with special attention to the aspects that are still controversial. The section is organized

in a, one could say, geographical way -from the upstream source, to the local changes, to downstream and global implications- rather than on a chapter-by-chapter basis. 


\section{The spreading of the Labrador Sea Water}

Recently significant attention has been given to the question of whether or not the product of convection in the Labrador Sea is exported via the DWBC. This route along the western boundary was traditionally thought to be the dominant pathway based on theoretical studies (Stommel and Arons (1960)) as well as tracer distributions (eg. Talley and McCartney (1982) and Smethie (1993)). In addition, transport estimates, velocity field and water mass properties measured over the continental slope in the southern Labrador Sea (Schott et al. (2004)) are remarkably similar to those observed at Line W (Toole et al. (2010)). However, recent work based on float trajectories suggested that the DWBC is only a minor contributor to the southward export of LSW (Fischer and Schott (2002) and Bower et al. (2009)). The evolution of the properties of the intermediate water at Line $\mathrm{W}$, suggests that the DWBC does play an important role in the transport of the LSW. The properties of the LSW are determined by the winter conditions at the Labrador Sea where the water is ventilated. These conditions during the late 80s and early 90s favored the formation of the densest LSW ever observed (Yashayaev (2007)). Along with the increased density, those recently ventilated waters had weaker than normal stratification (resulting from the vigorous mixing). The increased LSW density and weakened stratification were observed at Line W approximately 9 years later. In the late 90s, after a number of years where convection did not occur leading to the gradual restratification of the water in the central Labrador Sea, a much lighter and thinner layer of LSW was formed. This

lighter LSW also appeared at Line W, but in this case after a shorter period of time (5-6 years) than its deeper counterpart. The signature of recently ventilated LSW at Line W consisted of colder, fresher and less stratified water all consistent with the evolution of the properties at the central Labrador Sea. And not only the evolution of the properties of the water suggested the LSW was reaching the subtropics via the DWBC, the rate at which the signals spread from high- to mid-latitudes was in good agreement with changes in the strength of the DWBC itself.

The spreading of the LSW core appears to be related to the dynamics of the 
mean and the slowly varying part of the circulation, rather than to the synoptic velocity field that governs the float trajectories. The different nature of the mean and synoptic velocity fields can explain, at least in part, the apparent disagreement between float and tracer derived spreading pathways. This is clear from the analysis of simulated particle trajectories in Getzlaff et al. (2006) compared to Bower et al.'s float trajectories. Getzlaff et al. (2006) found that $90 \%$ of the particles that are exported southward do so in the DWBC when the mean circulation is considered, whereas only $25 \%$ of the floats that reach the subtropics do it on the DWBC according to Bower et al. (2009). One possible reason explaining this difference, is the 3-dimensional nature of the mean velocity field, compared to the 2 -dimensional eddy variability. The LSW core, like the mean circulation, experiences a large increase in depth (ranging from $500 \mathrm{~m}$ to $1000 \mathrm{~m}$ ) as it rounds the tail of the Grand Banks. Evidence of this drop was provided by Talley and McCartney (1982) based on the location of the potential vorticity minimum, and can also be seen in the deepening of the bottom velocity cores in the DWBC upstream (Pickart and Spall (2007)) and downstream (Schott et al. (2004)) from the Grand Banks. This drop is most likely the result of the interaction between the DWBC and the North Atlantic Current. Similar to what happens at the crossover between the DWBC and the Gulf Stream at Cape Hatteras (Hogg and Stommel (1985)), the DWBC slides down the slope in order to negotiate its passage into the subtropics while conserving PV. The floats due to their isobaric nature cannot follow this changes in depth, resulting into a larger percentage of floats being ejected from the DWBC at the tail of the Grand Banks. The eddies, on the other hand, are more 2-dimensional, and their behavior is well captured by the drift of Bower et al.'s (2009) isobaric floats.

That said, our view of the DWBC is not of a continuous conduit communicating information and water parcels from the subpolar to the subtropical North Atlantic. Our observations at Line W, like Bower et al.'s (2009) floats, showed frequent flow reversals, and its only as we average over time periods of 6 months or longer, that the DWBC emerges as a distinct current. The averaging is not just a mathematical tool, 
but represents the integrating nature of the ocean, the slow response to the rapidly varying forcing.

\section{The transport of the DWBC. Overflow or Labrador Sea Water driven?}

The LSW is only one of the constituents of the North Atlantic Deep Water. Waters originating at the Iceland-Scotland Overflow and Denmark Strait Overflow populate the deep layers below the LSW. The OW is formed by convection in the Nordic Seas and is colder and less stratified than the surrounding water when it has been recently ventilated. On its transit out of the Nordic Seas, over the sills then west into the Labrador Sea, both ISOW and DSOW entrain large amounts of the surrounding waters. The effect of this entrainment is particular strong in the ISOW, which is directly in contact with the dLSW and whose property time histories as a result of the strong mixing follow a similar evolution to those of the dLSW (Yashayaev et al. (2007)). The evolution of the DSOW is, due to the combination of atmospheric and circulation changes, opposite to that of the dLSW (Dickson et al. (1996)). The vertical structure of the potential vorticity field at Line $\mathrm{W}$ showed evidence of these coordinated changes in the OW and dLSW. We found that the dLSW and the ISOW layers expand at the expense of the DSOW and uLSW contraction.

However, changes in the atmospheric forcing at high latitudes do not only affect the properties of the dense water formed. According to modeling studies, the circulation also adjusts to the increase in deep water formation. The amplitude of this response in the circulation and what in particular it is sensitive to, are still unresolved issues. It is generally found in modeling studies that certain changes in the atmospheric forcing over the convective basins leads to enhanced dense water formation, which can then cause changes in the strength of the overturning circulation. Examples of this can be found in Gerdes and Koberle (1995), Mauritzen and Häkkinen (1999), Bailey et al. (2005), Böning et al. (2006), Zhang (2008) and Kohl and Stammer (2008) among others. However, there is little observational evidence on this subject. And in cases 
when data are available, they often contradict previous modeling results (Pickart and Spall (2007)). Our analysis of the 4-year Line W record (and 5.5 years of the central profiling mooring) showed that the transport of the DWBC, for both intermediate and deep waters, increased when the DSOW had a larger presence in the section, the layer of dLSW was reduced, and the uLSW, at least the fraction of it that is resolved by the Line $\mathrm{W}$ array, expanded. This increase in the transport was the result of a number of factors acting together. The first one, related to an increase in the width of the DWBC resulting from a southward displacement of the Gulf Stream axis, will be discussed in some more detail later. The second one, and more relevant to the water mass distribution, is the geostrophic response of the flow to what appears to be a larger flushing of OW, with a larger contribution from the deeper DSOW than from ISOW. The increased volume of OW pushes the isopycnals up, producing positive sea surface elevation and bottom pressure anomalies and drives a flow of $-5 \mathrm{~cm} / \mathrm{s}$ that affects the entire water column. A similar mechanism exists for the uLSW and dLSW as well, however the amplitude of the changes in the volume of LSW, in particular in the dLSW, appears to be smaller, and so is the circulation induced by them. When the phase relation between uLSW, dLSW and OW is taken into account, the circulation induced by the anomalies in the $\mathrm{OW}$ is one order of magnitude stronger than the circulation induced by either type of LSW and so the total transport is best tracked by changes in the OW.

Dickson et al. (1996) argued that the anti-phase relation between the OW and dLSW is a consequence of the difference in the conditions over the Nordic and Labrador Seas associated with a particular phase of the NAO, which favor the production of the one, and impede the production of the other. However, in the more recent work by Sarafanov et al. (2009), the authors suggested that the local conditions at the Labrador Sea may also play a role in the dynamics of the overflows. They found that changes in the DWBC transport, at the level of the OW, lagged changes in the Labrador Sea by 1-3 years, and hypothesized that changes in the production of LSW can alter the circulation and partially block the export of OW. Which of the 
two scenarios is the correct one? Most likely a combination of the two. Based on the amplitude of the signals that reach Line W, specifically the amplitude of the PV anomalies in the OW and dLSW, we believe that changes in the source of the OW must play a significant role. The total change in PV associated with the OW signal is much larger than the total change in PV in the dLSW, and therefore the expansion/contraction of the OW cannot be the sole result of the contraction/expansion of the dLSW layer. Changes in the source of the OW may be communicated more effectively to mid-latitudes, since the OW has a more direct route into the DWBC. The LSW is formed in the center of the basin and must be somehow transported into the boundary current in order for it to be exported. If diffusion plays a role in this transport, as proposed by Straneo et al. (2003), it is reasonable to expect that the signature of even the very strong convection in the Labrador Sea, will be diluted on its way to the boundary. In addition, the propagation of the signals associated with changes in the LSW and OW can be different too. Using a 1.5 layer model Yang and Joyce (2003) showed that perturbations originating in a boundary flow (OW) and the circulation induced by them propagate southward more efficiently than those associated with sources in the interior of a basin (LSW).

These variations in the transport of the DWBC that we observed at Line W may be partially compensated through changes in the interior. The amplitude of the fluctuations in the MOC at $26.5^{\circ} \mathrm{N}$ (Cunningham et al. (2007)) are smaller than the fluctuations in the transport of the DWBC observed at Line W. Although the observational record is not long enough yet to validate some of these results, modeling studies suggest that the variability in the MOC at shorter time scales is forced by the wind (Cabanes et al. (1996)). The amplitude of the MOC response to the wind forcing is larger in the interior than along the boundaries. Longer-term changes in the MOC, on the other hand, are related to changes in the dense water formation, and are transmitted to the subtropics mostly along the western boundary (Kohl and Stammer (2008)). The different time scales in the MOC response, as well as what part of the basin the effects of the response are felt stronger, may also explain some of 
the differences between deep water spreading pathways inferred from floats compared to tracer observations. The floats spread according to the full synoptic velocity field. Wind-forced motions are the dominant source of variability in this case. Tracers, on the other hand, see an integrated version of the rapidly changing synoptic fields. Throughout this thesis we have used EOF as a means to separate the slowly-varying part of the velocity field that is associated with the spreading of the tracer, PV in this case, rather than with the rapid fluctuations cause by the wind. The modes of variability described by the EOF captured only a fraction of the total variability. Thus the dense water spreading routes and times inferred from them are expected to be different from those inferred from float observations.

\section{Downstream from Line W. The Gulf Stream/DWBC inter- action.}

The analysis of surface temperature and surface geostrophic velocities performed in Chapter 2 showed that periods of intensified (weaker) southwestward flow in the Slope Water were accompanied by colder (warmer) than average water temperatures. Changes between the weak and the strong circulation phases occurred typically within periods of 2-3 years. Interestingly, the fluctuations in the circulation and properties of the Slope Water appeared to be related to changes in the Gulf Stream position, with colder than usual water and intensification of the flow preceding southward shifts of the Gulf Stream axis. The vertical structure of the velocity anomalies at Line W described in Chapter 4, as well as the flow induced by interior PV anomalies discussed

in Chapter 5, show the variability in the circulation in the Slope Water/DWBC area is rather barotropic. Thus, changes in the Slope's circulation are the surface expression of changes in circulation and properties of the water at depth. Therefore, the relation between the transport of the DWBC and the Gulf Stream separation proposed by Thompson and Schmitz (1989) may apply here, with the increase in the DWBC transport being the result of an increase in the export of recently ventilated OW and uLSW. 
To further demonstrate the relation between changes in the surface flow and changes in the deep circulation, we integrated the sum of the downstream velocity mode and mean velocity section from the Line W described in Chapter 4 (figure 4-11) between the $1000 \mathrm{~m}$ and the $3500 \mathrm{~m}$, the boundaries of the Slope Water in Chapter 2. The evolution of this inner-slope transport and changes in the altimeter-derived surface geostrophic velocity anomalies averaged east of $69^{\circ}$, where the direct effect of the Gulf Stream and its recirculation are offshore the $3500 \mathrm{~m}$ isobaths, compare relatively well (figure 6-1). Inner-slope transport fluctuations of 2Sv correspond to changes in the surface geostrophic flow of $5 \mathrm{~cm} / \mathrm{s}$. These transport changes may seem small compared to the 40Sv required in Thompson and Schmitz's (1989) two-layer model to induced a $100 \mathrm{~km}$ shift in the latitude of the Gulf Stream. Several factors can contribute to this difference. First, our estimate of the transport does not fully resolve the upper $1000 \mathrm{~m}$ of the water column, where the amplitude of the fluctuations in the flow could be several times larger than what we measure at depth. Secondly, the amplitude of the changes in the latitude of the Gulf Stream that are captured by surface velocity PC1 in Chapter 2 of this thesis, is approximately half of the latitude change discussed by Thompson and Schmitz (1989) (50-60km). Therefore a smaller transport change may be sufficient to explain the observed changes in the Gulf Stream path. And finally, as was shown by Spall (1996a), it is only the upper flow in the DWBC that interacts with the Gulf Stream. The deeper DWBC layers can flow relatively unmodified under the Gulf Stream. And so the dynamically relevant part of the transport prescribed by Taylor and Stephens (1998) is only just a fraction of the total.

However, the variability in the Gulf Stream path is more complex than the just described in-phase north-south shifts. The fraction of the variability explained by this dominant mode is time and space dependent. Its amplitude was large during the early part of the altimetric record (1993-2000), but it decreased in the more recent years. We also saw that at Line $\mathrm{W}$, the amplitude of the mode is low and the synoptic variability of the Gulf Stream position is better characterized by the second mode of 


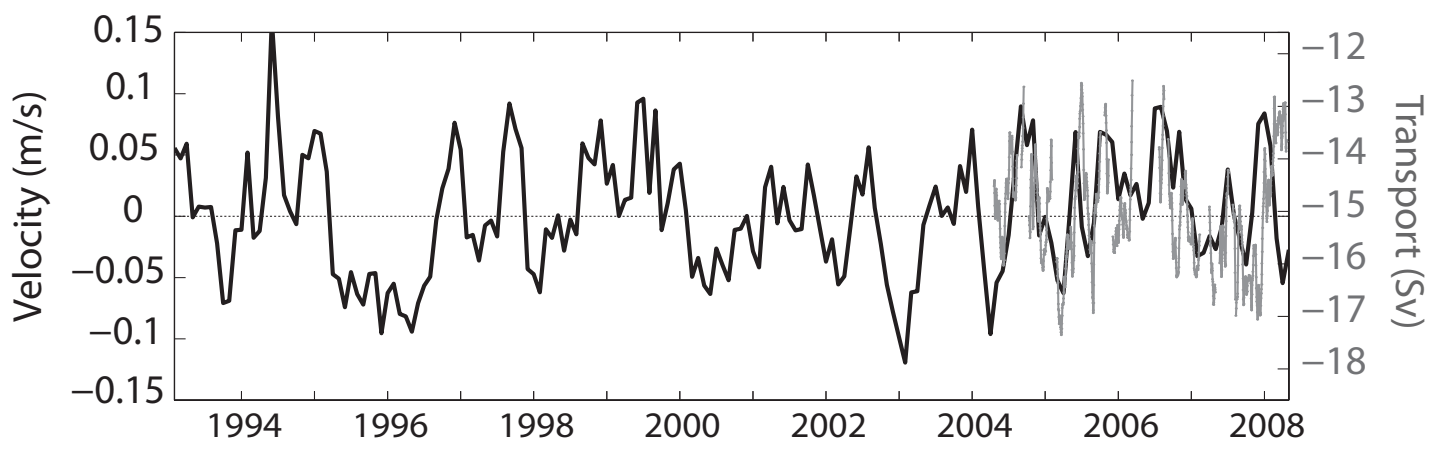

Figure 6-1: The altimeter-derived surface geostrophic velocity anomalies averaged in the eastern part of the Slope Water (east of $69^{\circ} \mathrm{W}$; black line) compare well with the inner-slope transport variability, shown in gray. Inner-slope transport anomalies are obtained by integrating the sum of the mean downstream velocity section (figure 4-5) and the leading EOF of the velocity described in section 4.5 (figure 4-11) between the 1000 and the $3500 \mathrm{~m}$ isobaths used in defining the Slope.

Gulf Stream path variability. The second mode is also a north-south oscillation but with a node approximately $200 \mathrm{~km}$ west of Line W. To the east and to the west of the node, changes in the latitude of the Gulf Stream path have opposite sign, which results in an overall change in the meridional tilt of the Stream. During the Line W observational period (2004-2008), the amplitude of this second mode was particularly large, and it appeared to be in phase with changes in the circulation and properties of the deep water.

Another question is, is the timing of the variability in the Gulf Stream path, Slope Water SST and velocity changes and spreading of the NADW along the DWBC consistent with one another? The answer is complicated, given the large number of elements that participate in the problem, and the variety in the time scales of the responses to each of them. The variability in the path of the Gulf Stream can be caused by a number of processes. It can be the result of changes in the wind forcing (eg. Gangopadhyay et al. (1992) and Taylor and Gangopadhyay (2001)). GS path variability can be associated with internal oscillations of the jet itself (Berloff et al. (2007)), and/or can be part of the response to changes in the thermohaline forcing. The latter is believed to consist of a fast wave response (Roussenov et al. (2008)), occurring mostly along the boundaries, followed by a slow advective response (Gerdes 
and Koberle (1995)). Both the wave and advective responses have the potential to interact with the wind driven circulation, and in particular with the Gulf Stream. The pressure perturbation at the front of the topographic waves generated by buoyancy forcing in the northern North Atlantic can induce changes in the layer thickness that modify the circulation near the Gulf Stream (Février et al. (2007)). Changes in the advective response might also occur, either by the Thompson and Schmitz (1989) mechanism (advection of thermocline interface by the DWBC flow), or by entrainment of the low PV water carried by the DWBC (Spall (1996b)). How to separate these different thermohaline-related mechanisms, and furthermore, how to separate thermohaline from direct adjustment to the wind field, given that changes in the thermohaline forcing at high latitudes are mostly driven by changes in the atmospheric forcing, is rather challenging. Additionally, the variety of indices used in the literature to characterize the atmospheric state and the Gulf Stream position does not make the exercise any easier.

In general, positive NAO periods are associated with high dLSW formation rates, while uLSW is formed during years when the NAO is low or negative. We saw in Chapter 4 that the largest transports in the DWBC are associated with recently ventilated uLSW, arriving at Line W 5 to 6 years after is formed in the Labrador Sea. The propagation of the SST signals within the Slope, on the other hand, appears to be faster. We found that changes in the Slope's SST that precede changes in the Gulf Stream position traveled between $62^{\circ} \mathrm{W}$ and Cape Hatteras, $1 / 4$ of the distance between Line $\mathrm{W}$ and the central Labrador Sea along the $3000 \mathrm{~m}$ isobath, in about one year. The difference in propagation speeds obtained from the 5-6 year spreading time of the LSW and the 4 years (for the same traveled distance) from the SST anomalies, can be due to several factors. First, it can be due to the residence time of the LSW in the Labrador Sea. A number of modeling studies have shown that after convection occurs, the transfer of newly formed LSW from the central Labrador Sea to the boundary current is not immediate, but can take a few years (eg. Straneo et al. (2003) and Deshayes et al. (2007)). The addition of this residence time, of typically 
3 years in model calculations but in the real ocean probably a function of the where in the basin convection occurs, will result in a spreading rate comparable to those derived in the surface analysis. Other factors that can contribute to the apparent slower spreading rates of LSW are mixing with high PV surrounding water, as well time spent by the LSW in the recirculations. Both of these processes were found to play an important role when explaining differences between CFC-derived LSW spreading times and direct velocity measurements in the DWBC (Smethie (1993)).

Nevertheless, one must be careful in establishing possible connections between atmospheric forcing, thermohaline and wind driven circulation. The Line $\mathrm{W}$ moored array was in the water during a period of time when the NAO index alternated between weakly positive and weakly negative phases. The longer altimeter and SST records, showed that from the mid 90s to the early 2000s the circulation and temperature of the western North Atlantic underwent major changes. The relation between these changes and the NAO index is clear during the extreme events, but becomes more diffuse towards the end of the record. The relative importance of the different elements of the climate system may be different at different times. The ocean may transition between different regimes, in which its sensitivity to the forcing represented by the NAO changes, as Joyce (2002) suggested. For that reason, long term monitoring programs, such as Line W, are crucial to determine what these regimes are, and what aspects of the climate system are more likely to be affected by them.

\section{A note on monitoring}

The coordinated changes in the deep and intermediate water we observed at Line W have an impact in the surface circulation in the continental slope. Associated with the expansion and contraction of individual water masses, we also saw changes in the surface elevation as well as the bottom pressure. The amplitude of the surface flow induced by these deep anomalies is larger ( $5 \mathrm{~cm} / \mathrm{s}$ approx.) in the deeper part of the continental slope, offshore from the $3500 \mathrm{~m}$ isobath just above the DSOW (figure 6-2). In the inner slope, between the 1000 and $3500 \mathrm{~m}$ isobaths the velocity induced by the 

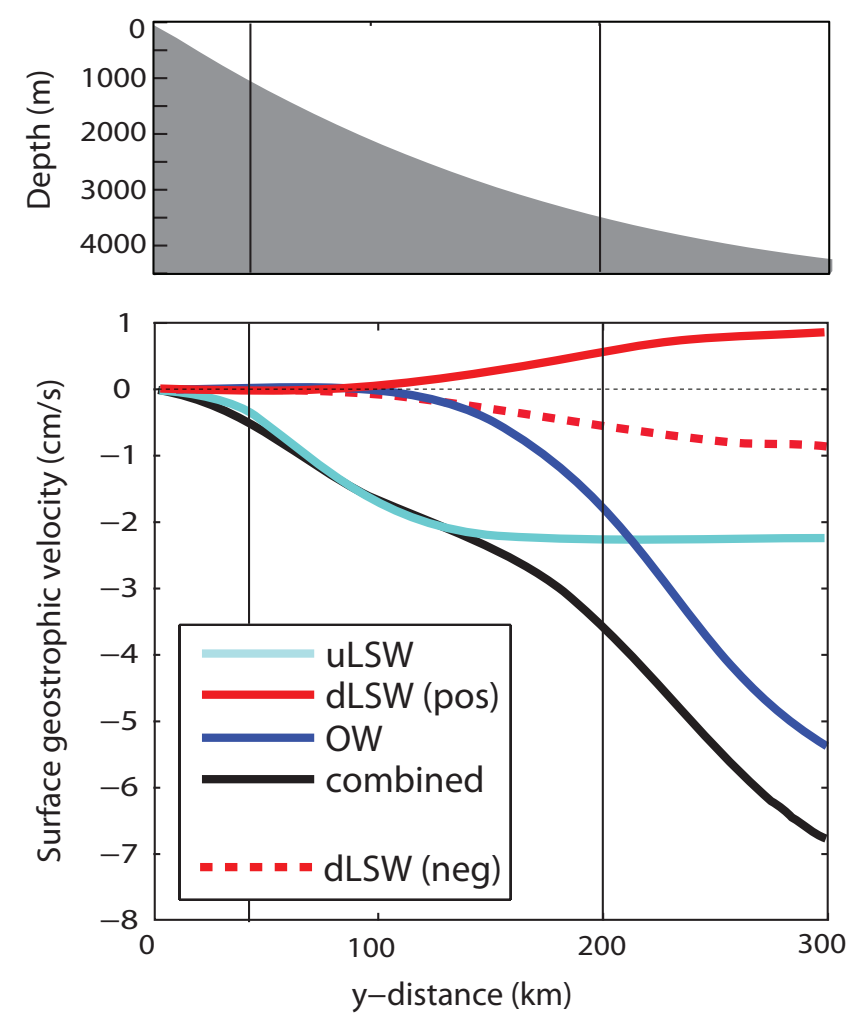

Figure 6-2: The panel on the bottom shows surface geostrophic velocities induced by the negative uLSW PV anomaly (light blue), positive dLSW (solid red), negative OW PV anomaly (dark blue), and the combination of the three (black) (from figure 5-10). Also shown is the velocity associated with a negative dLSW PV anomaly (dashed red). The horizontal axis shows distance increasing offshore out to $300 \mathrm{~km}$. And the vertical black lines represent the approximate location of the $1000 \mathrm{~m}$ and $3500 \mathrm{~m}$ isobaths in the idealized continental slope shown in the upper panel.

combined PV anomalies in the uLSW, dLSW and OW, has amplitudes of $2-3 \mathrm{~cm} / \mathrm{s}$. In contrast with the outer slope, these velocities in the shallow part of the section are associated with uLSW PV anomalies, the effect of OW PV anomalies is stronger further offshore.

The comparison between the velocities induced by the subsurface distribution of PV between the $1000 \mathrm{~m}$ and $3500 \mathrm{~m}$ isobaths and the altimeter-derived surface geostrophic velocities in the Slope Water described in Chapter 2, is encouraging. The predicted amplitude from the PV-induced velocities is somewhere between the altimeter-derived surface geostrophic velocities averaged over the entire slope (ca. $4 \mathrm{~cm} / \mathrm{s}$, in figure 2-7), and the velocity mode of the joint EOF of the Slope's SST 
and velocity anomalies shown (1-2cm/s, in figure 2-8). The spatial pattern of the velocity mode, from the joint analysis of SST and velocity anomalies in the Slope Water, indicates that the optimal place to monitor the DWBC using altimetry may be east of Line W. In the region between Cape Hatteras and Line W, the variability in the circulation is directly affected by changes in the Gulf Stream position (a large negative anomaly in the flow indicating a southward shift of the Gulf Stream axis). The amplitude of the Gulf Stream signal is larger than the changes in the Slope Water, thus the surface velocity signal of the DWBC might be better observed further east where the Slope is sufficiently separated from the Gulf Stream. The amplitude (and sign) of the variability in the Slope's velocity for the region east of $69^{\circ}$ only (not shown here), is similar to the amplitude of the signal over the entire Slope, and the correlation with the SST anomalies significant too. And based on the time scales of the deep variability inferred from Line $W$, these surface signals that we see must be coherent over long distances, and could be monitored from space.

\section{Future work}

Based on the results from the Line W observations, one of the questions that arises is whether or not the observed relationships between upper, intermediate and deep water masses are exclusive to Line $\mathrm{W}$ or representative of other locations as well. The phase relation between uLSW and OW could be coincidental, just the result of different formation times, spreading rates and traveled distances acting together. In order to test the validity of the results presented here at other locations, the Line $\mathrm{W}$ array could be complemented with a series of single moorings in the DWBC upstream from Line W. Our observations suggest that the $3000 \mathrm{~m}$ is a good location to monitor changes in the water mass composition as well as changes in the flow. To exploit the correlation between surface and deep variability, the location of the moorings in the along-isobath direction could be chosen based on the location of the altimeter tracks. In addition to address the question of whether the Line W observations are representative of other locations, the additional moorings could be used to explore the 
continuity of the signals of thermohaline origin traveling southward along the western boundary.

Regarding the PV-inversion experiments, some simple 3-dimension experiments could be carried out. The addition of the third dimension would allow for density changes in the along-isobath direction and the corresponding cross-stream velocity component. The cross-stream velocity component is the one responsible for the advection of the Gulf Stream front by the DWBC in the Thompson and Schmitz's (1989) model. The $3 \mathrm{~d}$-inversion would therefore provide a simple way to test this mechanism for the DWBC/Gulf Stream interaction based on PV anomalies with scalings derived from the observations, the same way it was done for the 2-dimensional problem. 


\section{Bibliography}

Bacon, S., 1998: Decadal variability in the outflow from the nordic seas to the deep atlantic ocean. Nature, 394, 871-874.

Bailey, D. A., P. B. Rhines, and S. Hakkinen, 2005: Formation and pathways if the North Atlantic Deep Water in a couple ice-ocean model of the Arctic-North Atlantic Oceans. Climate Dynamics, 25, 497-516, doi:10.1007/s00382-005-0050-3.

Berloff, P., A. M. Hogg, and W. Dewar, 2007: The Turbullent Oscillator: The Mechanics of Low-Frequency Variability of the Wind-Driven Ocean Gyres. Journal of Physical Oceanography, in press.

Böning, C. W., F. O. Bryan, W. R. Holland, and R. Doscher, 1996: Deep-water formation and meridional overturning in a high-resolution model of the north atlantic. Journal of Physical Oceanography, 26, 1142-1164.

Böning, C. W., M. Scheinert, J. Dengg, A. Biastoch, and A. Funk, 2006: Decadal variability of subpolar gyre transport and its reverberation in the North Atlantic overturning. Geophysical Research Letters, 33 (L21S01).

Bower, A. S., 1989: Potential Vorticity Balances and Horizontal Divergence along Particle Trajectories in Gulf Stream Meanders East of Cape Hatteras. Journal of Physical Oceanography, 19, 1669-1681.

Bower, A. S. and H. D. Hunt, 2000: Lagrangian Observations of the Deep Western Boundary Current in the North Atlantic Ocean. Part I: Large-scale pathways and spreading rates. Journal of Physical Oceanography, 30, 764-783.

Bower, A. S., M. S. Lozier, S. F. Gary, and C. W. Böning, 2009: Interior pathways of the North Atlantic meridional overturning circulation. Nature, 459.

Bretherton, F. P., 1966: Critical layer instability in baroclinic flows. Quarterly Journal of the Royal Meteorological Society, 92, 325-334.

Brown, O. B., P. C. Cornillon, S. R. Emmerson, and H. M. Carle, 1986: Gulf stream warm rings: a statistical study of their behavior. Deep-Sea Research, 33, 1459-1473. 
Bryden, H. L., W. E. Johns, and P. M. Saunders, 2005: Deep western boundary current east of Abaco: mean structure and transport. Journal of Marine Research, $63(1), 35-57$.

Cabanes, C., T. Lee, and L.-L. Fu, 1996: Mechanisms of interannual variations of the meridional overturning circulation of the north atlantic ocean. Journal of Physical Oceanography, 38, 467-480.

Charney, J. G. and M. E. Stern, 1962: On the stability of internal baroclinic jets in a rotating atmosphere. Journal of the Atmospheric Sciences, 19, 159-172.

Clarke, R. A. and J.-C. Gascard, 1983: Formation of labrador sea water. part i: Large-scale processes. Journal of Physical Oceanography, 13, 1764-1778.

Coetlogon, C. D., C. Frankignoul, M. Bentsen, C. Delon, H. Haak, S. Masina, and N. Pardaens, 2006: Gulf Stream variability in five oceanic general circulation models. Journal of Physical Oceanography, 36, 2119-2135.

Cronin, M. and D. R. Watts, 1996: Eddy-mean flow interaction in the gulf stream at 68w. part i: Eddy energetics. Journal of Physical Oceanography, 26, 2107-2131.

Csanady, G. T. and P. Hamilton, 1988: Circulation of slopewater. Continental Shelf Research, 8, 565-624.

Cunningham, S. A., et al., 2007: Temporal variability of the atlantic meridional overturning circulation at 26.5n. Science, 317, doi:10.1126/science.1141304.

Curry, R. G. and M. S. McCartney, 2001: Ocean gyre circulation changes associated with the north atlantic oscillation. Journal of Physical Oceanography, 31, 33743400 .

Davis, C. A. and K. A. Emanuel, 1991: Potential vorticity diagnostics of cyclogenesis. Monthly Weather Review, 119, 1929-1953.

Deshayes, J., C. Frankignoul, and H. Drange, 2007: Formation and export of deep water in the Labrador and Irminger Seas in a GCM. Deep-Sea Research I, 54, $510-532$.

Dickson, R., J. Lazier, J. Meincke, P. Rhines, and J. Swift, 1996: Long-term coordinated changes in teh convective activity of the north atlantic. Progress in Oceanography, 38, 241-295.

Eden, C. and J. Willebrand, 2001: Mechansism of interannual to decadal variability of the north atlantic circulation. Journal of Climate, 14, 2266-2280.

Ezer, T. and G. L. Mellor, 1992: A numerical study of the variability and the separation of the gulf stream induced by surface atmospheric forcing and lateral boundary flows. Journal of Physical Oceanography, 22, 660-682. 
Février, S., J. Sirven, and C. Herbaut, 2007: Interaction of a coastal kelvin wave with the mean state in the gulf stream separation area. Journal of Physical Oceanography, 37, 1729-1444.

Fischer, J. and F. A. Schott, 2002: Labrador Sea Water Tracked by Profiling FloatsFrom the Boundary Current into the Open North Atlantic. Journal of Physical Oceanography, 32, 573-584.

Flagg, C. N., M. Dunn, D.-P. Wang, H. T. Rossby, and R. L. Benway, 2006: A study of the currents of the outer shelf and upper slope from a decade of shipboard adcp observations in the middle atlantic bight. Journal of Geophysical Research, 111 (C06003).

Frankignoul, C., C. D. Coetlogon, T. M. Joyce, and S. Dong, 2001: Gulf Stream variability and Ocean-Atmosphere Interactions. Journal of Physical Oceanography, 31, 3516-3529.

Frantantoni, P. S. and R. S. Pickart, 2003: Variability of the shelf break jet in the Middle Atlantic Bight: Internally or externally forced? Journal of Geophysical Research, 108 (C25, 3166), doi:10.1029/2002JC001326.

Gangopadhyay, A., P. Cornillon, and R. D. Watts, 1992: A test of the parsons-veronis hypothesis on the separation of the gulf stream. Journal of Physical Oceanography, 22, 1286-1301.

Gascard, J.-C. and R. A. Clarke, 1983: Formation of labrador sea water. part ii: Mesoscale and smaller-scale processes. Journal of Physical Oceanography, 13, 17791797.

Gerdes, R. and C. Koberle, 1995: On the influence of dwow in a numerical model of the north atlantic general circulation. Journal of Physical Oceanography, 25, 2624-2642.

Getzlaff, K., C. W. Böning, and J. Dengg, 2006: Lagrangian perspectives of deep water export from the subpolar north atlantic. Geophysical Research Letters, 33 (L21S08), doi:10.1029/2006GL026470.

Häkkinen, S. and P. B. Rhines, 2004: Decline of Subpolar North Atlantic Circulation during de 1990s. Science, 304, 555-559.

Häkkinen, S. and P. B. Rhines, 2009: Shifting surface currents in the northern north atlantic ocean. Journal of Geophysical Research, 114, doi:10.1029/2008JC004883.

Halkin, D. and T. Rossby, 1985: The structure and transport of the gulf stream at $73^{\circ} \mathrm{w}$. Journal of Physical Oceanography, 15, 1439-1452. 
Hallberg, R. and P. Rhines, 1996: Buoyancy-driven circulation in an ocean basin with isopycnals intersecting the sloping boundary. Journal of Physical Oceanography, 26, 913-940.

Hogg, N. G. and H. Stommel, 1985: On the relation between the deep circulation and the Gulf Stream. Deep-Sea Reserach, 32, 1182-1193.

Horel, J. D., 1984: Complex principal component analysis: Theory and examples. Journal of Climate and Applied Meteorology, 23, 1660-1673.

Hoskins, B. J., M. E. McIntyre, and A. W. Robertson, 1985: On the use and significance of isentropic potential vorticity maps. Quarterly Journal of the Royal Meteorological Society, 111, 877-946.

Hurlburt, H. E. and P. J. Hogan, 2008: The gulf stream pathway and the impacts of the eddy-driven abyssal circulation and the deep western boundary current. Dynamics of Atmospheres and Oceans, 45, 71-101, doi:10.1016/j.dynatmoce.2008.06.002.

Hurrell, J. W., 1995: Decadal trends in the north atlantic oscillation: Regional temperatures and precipitation. Science, 269, 676-679.

Johns, W. E., T. J. Shay, J. M. Bane, and D. R. Watts, 1995: Gulf stream structure, transport and recirculation near 68w. Journal of Geophysical Research, 100, 817838.

Joyce, T., et al., 1983: Rapid evolution of a gulf stream warm-core ring. Nature, 308, 837-840, doi:10.1038/308837a0.

Joyce, T. M., 2002: One hundred plus years of wintertime climate variability in the eastern united states. Journal of Climate, 15, 1076-1087.

Joyce, T. M., C. Deser, and M. Spall, 2000: The Relation between Decadal Variability of Subtropical Mode Water and the North Atlantic Oscillation. Journal of Climate, 13, 2550-2569.

Joyce, T. M., J. Dunworth-Baker, R. S. Pickart, D. Torres, and S. Waterman, 2005: On the Deep Western Boundary Current south of Cape Cod. Deep-Sea Research II, 52, 615-625.

Joyce, T. M. and R. Zhang, 2010: On the path of the gulf stream and the atlantic meridional overturning circulation. Journal of Climate, 23, 3146-3154.

Kelly, K. A., M. J. Caruso, and S. Singh, 1996: Observations of atmosphere-ocean coupling in midlatitude. Journal of Geophysical Research, 101, 6295-6312.

Kelly, K. A. and S. T. Gille, 1990: Gulf Stream Surface Transport and Statistics at 69w from the Geosat altimeter. Journal of Geophysical Research, 96, 3149-2161. 
Kohl, A. and D. Stammer, 2008: Variability of the Meridional Overturning in the North Atlantic from the 50-Year GECCO State Estimation. Journal of Physical Oceanography, 38, 1913-1930.

Koltermann, K. P., A. V. Sokov, V. P. Terescenlov, S. A. Dobroliubov, K. Lorbacher, and A. Sy, 1999: Decadal changes in the thermohaline circulation of the north atlantic. Deep-Sea Research II, 46, 109-138.

Lapeyre, G. and P. Klein, 2006: Dynamics of the upper oceanic layers in terms of surface quasigeostrophy theory. Journal of Physical Oceanography, 36, 165-176.

Lazier, J. R. N., 1980: Oceanographic conditions at ocean weather ship bravo, 19641974. Atmosphere-Ocean, 18 (3), 227-238.

Lee, T. and P. Cornillon, 1995: Temporal variation of meandering intensity and domain-wide lateral oscillations of the Gulf Stream. Journal of Geophysical Research, 100, 13 603-13613.

Lee, T. N., W. E. Johns, R. J. Zantopp, and E. R. Fillenbaum, 1996: Moored observations of Western Boundary Current Variability and Thermohaline Circulation at $26.5 \mathrm{~N}$ in the Subtropical North Atlantic. Journal of Physical Oceanography, 26, 962-983.

Macrander, A., U. Send, H. Vladimarsson, S. Jonsson, and R. H. Käse, 2005: Interannual changes in the overflow from the nordic seas into the atlantic ocean through denmark strait. Geophysical Research Letters, 32, doi:10.1029/2004GL021463.

Marshall, J., C. Hill, L. Perelman, and A. Adcroft, 1997: Hydrostatic, quasihydrostatic, and nonhydrostatic ocean modeling. Journal of Geophysical Research, 102, 5733-5752.

Mauritzen, C. and S. Häkkinen, 1999: On the relationship between dense water formation and the Meridional Overturning Cell in the North Atlantic Ocean. DeepSea Research I, 46, 877-894.

McCartney, M. S., 1992: Recirculating components to the deep boundary current of the northern north atlantic. Progress in Oceanography, 29, 283-383.

McIntyre, M. E., 2003: New Encyclopedia of Atmospheric Sciences, Vol. 2. Academic Press, 680-685 pp.

Mertz, G. and D. Wright, 1992: Interpretations of the jebar term. Journal of Physical Oceanography, 22, 301-305.

Molinari, R. L., R. A. Fine, W. D. Wilson, R. G. Curry, J. Abell, and M. S. McCartney, 1998: The arrival of recently formed Labrador sea water in the Deep Western Boundary Current at 26.5N. Geophysical Research Letters, 25 (13), 2249-2252. 
North, G. F., T. L. Bell, R. F. Cahalan, and F. J. Moeng, 1982: Sampling Errors in teh Estimation of Empirical Orthogonal Functions. Monthly Weather Review, 110, 699-706.

North, G. R., 1984: Empirical orthogonal functions and normal modes. Journal of the Atmospheric Sciences, 41 (5), 879-887.

Paillet, J., B. L. Cann, X. Carton, Y. Morel, and A. Serpette, 2002: Dynamics and evolution of a northern meddy. Journal of Physical Oceanography, 32, 55-79.

Pedlosky, J., 1979: Geophysical Fluid Dynamics. Springer Verlag.

Pena-Molino, B. and T. M. Joyce, 2008: Variability in the Slope Water and its relation to the Gulf Stream path. Geophysical Research Letters, 35.

Petrie, B. and K. Drinkwater, 1993: Temperature and salinity variability on the scotian shelf and in the gulf of maine 1945-1990. Journal of Geophysical Research, 98 (C11), 20079-20089.

Pickart, R. S., 1992: Water mass components of the North Atlantic deep western boundary current. Deep-Sea Research, 39, 1553-1572.

Pickart, R. S., 1994: Interaction of the Gulf Stream and the Deep Western Boundary Current where they cross. Journal of Geophysical Research, 99, 25 155-25164.

Pickart, R. S., 1995: Gulf Stream-Generated Topographic Rossby Waves. Journal of Physical Oceanography, 25, 574-586.

Pickart, R. S., W. M. S. Jr., J. R. N. Lazier, E. P. Jones, and W. J. Jenkins, 1996: Eddies of newly formed upper Labrador Sea Water. Journal of Geophysical Research, 101 (C9), $20711-20726$.

Pickart, R. S. and W. M. Smethie, 1993: How Does the Deep Western Boundary Current Cross the Gulf Stream? Journal of Physical Oceanography, 23, 2602-2616.

Pickart, R. S. and W. M. Smethie, 1998: Temporal evolution of the deep western boundary current where it enters the sub-tropical domain. Deep-Sea Research Part I, 45, 1053-1083.

Pickart, R. S. and M. A. Spall, 2007: Impact of Labrador Sea Convection on the North Atlantic Meridional Overturning Circulation. Journal of Physical Oceanography, 37, 2207-2227.

Pickart, R. S., M. A. Spall, and J. R. N. Lazier, 1997: Mid-depth ventilation in the western boundary current system of the sub-polar gyre. Deep-Sea Research I, 44 (6), 1025-1054. 
Pickart, R. S. and D. R. Watts, 1990: Deep Western Boundary Current Variability at Cape Hatteras. Journal of Marine Research, 48, 765-791.

Reynolds, R. W., T. M. Smith, C. Liu, D. B. Chelton, K. S. Casey, and M. G. Schlax, 2006: Daily High-resolution Blended Analyses. Journal of Climate, In press.

Rhein, M., D. Kieke, and R. Steinfeldt, 2007: Ventilation of the Upper Labrador Sea Water, 2003-2005. Geophysical Research Letters, 34.

Rhein, M., et al., 2002: Labrador Sea Water: Pathways, CFC Inventory, and Formation Rates. Journal of Physical Oceanography, 32, 648-665.

Rio, M. H. and F. Hernandez, 2004: A mean dynamic topography computed over the world ocean from altimetry, un situ measurments, and a geoid model. Journal of Geophysical Research, 109 (C12032), doi:10.1029/2003JC002226.

Rossby, T., 1999: On gyre interactions. Deep-Sea Research, Part II, 46, 139-164.

Rossby, T. and R. L. Benway, 2000: Slow Variations in mean path of the Gulf Stream east of Cape Hatteras. Geophysical Research Letters, 27, 117-120.

Rossby, T. and E. Gottlieb, 1998: The /emphOleander Project: Monitoring the Variability of the Gulf Stream and Adjacent Waters between new Jersey and Bermuda. Bulletin of the American Meteorological Society, 79, 5-18.

Roussenov, V. M., R. G. Williams, C. W. Hughes, and R. J. Bingham, 2008: Boundary wave communication of bottom pressure and overturning changes for the north atlantic. Journal of Geophysical Research, 113, doi:10.1029/2007JC004501.

Sarafanov, A., A. Falina, H. Mercier, P. Lherminier, and A. Sokov, 2009: Recent changes in the greenland-scotland overflow-derived water transport inferred from hydrographic observations in the southern irminger sea. Geophysical Research Letters, 36, doi:10.1029/2009GL038385.

Schott, F. A., J. Fischer, M. Wibaux, M. Dengler, and R. Zantopp, 2006: Variability of the Deep Western Boundary Current east of the Grand Banks. Geophysical Research Letters, 33, L21S07.

Schott, F. A., R. Zantopp, L. Stramma, M. Dengler, J. Fischer, and M. Wibaux, 2004: Circulation and Deep-Water Export at the Western Exit of the Subpolar North Atlantic. Journal of Physical Oceanography, 34, 817-843.

Silverthorne, K. E. and J. M. Toole, 2009: Seasonal kinetic energy variability of near-inertial motions. Journal of Physical Oceanography, 39, 1035-1049, doi: 10.1175/2008JPO3920.1.

Smethie, W. M., 1993: Tracing the thermohaline circulation in the western North Atlantic using chlorofluorocarbons. Progress in Oceanography, 31, 51-99. 
Smethie, W. M. and J. N. Smith, 2010: ${ }^{129}$ I transport in the deep western boundary current $(\mathrm{dwbc})$ in the north atlantic. paper presented at the Second Meeting of the U.S. AMOC Science Team, Miami, USA.

Spall, M. A., 1996a: Dynamics of the Gulf Stream/Deep Western Boundary Current Crossover. Part I: Entrainment and Recirculation. Journal of Physical Oceanography, 26, 2152-2168.

Spall, M. A., 1996b: Dynamics of the Gulf Stream/Deep Western Boundary Current Crossover. Part II: Low-Frequency Internal Oscillations. Journal of Physical Oceanography, 26, 2169-2182.

Stommel, H., 1948: The westward intensification of wind-driven ocean currents. Trans. of the American Geophysical Union, 29, 202-206.

Stommel, H., 1958: The abyssal circulation. Deep-Sea Research (Letters), 5, 80-82.

Stommel, H. and A. B. Arons, 1960: On the abyssal circulation of the world ocean-i. stationary planetary flow patterns on a sphere. Deep-Sea Research, 19, 707-718.

Stramma, L., D. Kieke, M. Rhein, F. Schott, I. Yashayaev, and K. P. Koltermann, 2004: Deep water changes at the western boundary of the subpolar North Atlantic during 1996 to 2001. Deep-Sea Research I, 51, 1033-1056.

Straneo, F., R. S. Pickart, and K. Lavender, 2003: Spreading of Labrador sea water: an advective-diffusive study based on Lagrangian data. Deep-Sea Research I, 50, 701-719.

Swallow, J. and L. Worthington, 1961: An observation of a deep countercurrent in the western North Atlantic. Deep-Sea Research, 8, 1-19.

Swift, J. H., K. Aagaard, and S.-A. Malmberg, 1980: The contribution of the denmark strait overflow to the deep north atlantic. Deep Sea Research Part A., 27, 29-42, doi:10.1016/0198-0149(80)90070-9.

Talley, L. D. and M. S. McCartney, 1982: Distribution and circulation of Labrador Sea water. Journal of Physical Oceanography, 12, 1189-1204.

Talley, L. D., J. L. Reid, and P. E. Robbins, 2003: Data-based meridional overturning streamfunctions for the globar ocean. Journal of Climate, 16, 3213-3226.

Taylor, A. H. and A. Gangopadhyay, 2001: A simple model of interannual displacements of the gulf stream. Journal of Geophysical Research, 106, 13 849-13860.

Taylor, A. H. and J. A. Stephens, 1998: The north atlantic oscillation and the latitude of the gulf stream. Tellus, 50A, 134-142. 
Thompson, J. D. and W. J. Schmitz, 1989: A Limited Area Model of the Gulf Stream: Desing, Initial Experiments and Model-Data Intercomparison. Journal of Physical Oceanography, 19, 791-814.

Thompson, R., 1971: Topographic rossby waves at a site north of the gulf stream. Deep-Sea Research, 18, 1-19.

Thompson, R. and J. R. Luyten, 1976: Evidence for bottom-trapped topographic rossby waves from signel moorings. Deep-Sea Research, 23, 629-635.

Toole, J. M., R. G. Curry, T. M. Joyce, M. McCartney, and B. P. na Molino, 2010: Transport of the North Atlantic Deep Western Boundary Current about 39N, 70W: 2004-2008. Deep Sea-Research, submitted.

Torrence, C. and G. P. Compo, 1998: A practical guide to wavelet analisys. Bulletin of the American Meteorological Society, 79 (1), 61-78.

Våge, K., et al., 2009: Surprising return of deep convection to the subpolar north atlantic ocean in winter 2007-2008. Nature Geoscience, 2, 67-72, doi: $10.1038 /$ ngeo382.

Vallis, G. K., 1996: Potential vorticity inversion and balance equations of motion for rotating and stratified flows. Quarterly Journal of the Royal Meteorological Society, 122, 291-322.

Vaughan, S. L. and R. L. Molinari, 1997: Temperature and Salinity Variability in the Deep Western Boundary Current. Journal of Physical Oceanography, 749-761.

von Storch, H. and G. Hannoschock, 1985: Statistical aspects of estimated principal vectors (eofs) based on small sample sizes. Journal of Climate and Applied Meteorology, 24, 716-724.

von Storch, H. and F. W. Zwiers, 1999: Statistical Analysis in Climate Research. Cambridge University Press.

Watts, D. R. and W. E. Johns, 1982: Gulf Stream Meanders: Observations on Propagation and Growth. Journal of Geophysical Research, 87, 9467-9476.

Worthington, L. V., 1976: On the North Atlantic Circulation. The Johns Hopkins Oceanographic Studies.

Wunsch, C., 2006: Discrete Inverse Sstate Estimation Problems. Cambridge University Press.

Wunsch, C. and D. Stammer, 1998: Satellite altimetry, the marine geoid, and the oceanic general circulation. Annual Review of Earth and Planetary Sciences, 26, 219-253. 
Yang, J. and T. M. Joyce, 2003: How do high-latitude North Atlantic climate signals the crossover between the Deep Western Boundary Current and the Gulf Stream? Geophysical Research Letters, 30.

Yashayaev, I., 2007: Hydrographic changes in the Labrador Sea, 1960-2005. Progress in Oceaongraphy, 73, 242-276.

Yashayaev, I., M. Bersch, and H. M. van Aken, 2007: Spreading of the Labrador Sea Water to the irminger and iceland basins. Geophysical Research Letters, 34 (L10602).

Yashayaev, I. and J. W. Loder, 2009: Enhanced production of labrador sea water in 2008. Geophysical Research Letters, 36 (L01606), doi:10.1029/2008GL036162.

Yu, L., R. A. Weller, and B. Sun, 2004: Improving latent and sensible heat flux estimates for the Atlantic Ocean (1988-1999) by a synthesis approach. Journal of Climate, 17, 373-393.

Zhang, R., 2008: Coherent surface-subsurface fingerprint of the atlantic meridional overturning circulation. Geophysical Research Letters, 35, doi: 10.1029/2008GL035463.

Zhang, R. and G. K. Vallis, 2006: Impact of Great Salinity Anomalies on the LowFrequency Variability of the North Atlantic Climate. Journal of Climate, 19, 470482.

Zhang, R. and G. K. Vallis, 2007: The role of bottom vortex stretching on the path of the North Atlantic Western Boundary Current and the Northern Recirculation Gyre. Journal of Physical Oceanography, 37, 2053-2080, doi:10.1175/JPO3102.1. 